Portland State University

PDXScholar

$1-1-2011$

\title{
Traffic Congestion Mitigation as an Emissions Reduction Strategy
}

Alexander York Bigazzi

Portland State University

Follow this and additional works at: https://pdxscholar.library.pdx.edu/open_access_etds Let us know how access to this document benefits you.

\section{Recommended Citation}

Bigazzi, Alexander York, "Traffic Congestion Mitigation as an Emissions Reduction Strategy" (2011). Dissertations and Theses. Paper 131.

https://doi.org/10.15760/etd.131

This Thesis is brought to you for free and open access. It has been accepted for inclusion in Dissertations and Theses by an authorized administrator of PDXScholar. Please contact us if we can make this document more accessible: pdxscholar@pdx.edu. 
Traffic Congestion Mitigation as an Emissions Reduction Strategy

by

\begin{abstract}
Alexander York Bigazzi
Master of Science

in

Civil and Environmental Engineering

Thesis Committee:

Miguel Figliozzi, Chair

Kelly Clifton

Christopher Monsere
\end{abstract}

Portland State University

(C) 2011 


\begin{abstract}
Policy-makers, transportation researchers, and activists often assume that traffic congestion mitigation results in reduced vehicle emissions without proper justification or quantification of the benefits. If congestion mitigation is going to be tied to air quality goals, a better understanding of the impacts of traffic congestion on motor vehicle emissions is needed. This research addresses that need by investigating under which circumstances the commonly held assumption linking congestion mitigation to emissions reductions is valid.
\end{abstract}

We develop and apply a mathematical framework to study the trade-offs between vehicle efficiency and travel demand that accompany travel speed changes. While the exact relationships among emissions, travel speed, and travel demand vary with location and pollutant, several consistent results arise. The potential for marginal emissions rate reductions through average travel speed adjustments is small for speeds between about 25 and $70 \mathrm{mph}$. Emissions rate sensitivity to speed increases with the fraction of heavy-duty vehicles and for certain pollutants (gaseous hydrocarbons and particulate matter), and decreases with the fraction of advanced-drivetrain vehicles, such as electric and gaselectric hybrid vehicles.

But travel volume is also a key consideration for the total emissions impacts of congestion and congestion mitigation. While travel speed increases are generally expected to increase efficiency, they are also expected to increase vehicle travel volume as a result of induced demand. To explore efficiency and volume trade-offs we look at 
emissions break-even conditions for average speed and travel demand elasticity. Depending on the pollutant and the vehicle fleet, total emissions are only expected to decrease with increasing travel speed for initial conditions of both low demand elasticity and low average speed. Thus, higher levels of congestion do not necessarily increase emissions, nor will congestion mitigation inevitably reduce emissions. This result includes projects that seek to increase vehicle throughput from existing roadway supply through better traffic management and operations. Congestion mitigation through reduced vehicle volumes, on the other hand, presents the opportunity for additive emissions benefits through efficiency improvements and total Vehicle Miles Traveled (VMT) reductions.

Comparing capacity-based congestion mitigation strategies with alternative emissions reduction strategies we show that where emissions reductions are possible through speed increases, the emissions benefits are likely to be more easily and costeffectively attained by other strategies. A sketch analysis of vehicle-class segregated facilities shows that truck-only lane strategies consistently out-perform generalpurpose/mixed-flow lane strategies in terms of emissions reductions.

An analysis of several congestion-related performance measures shows that for reflecting emissions impacts, VMT is an essential component of performance. Thus, alternative congestion metrics such as total/excess travel distance and travel time are preferable emissions performance indicators to speed or distance-normalized delay. The 
Travel Time Index, in particular, poorly reflects emissions changes on congested roadways.

This thesis offers several original contributions to the body of knowledge regarding congestion and emissions. First, it describes a parsimonious conceptual framework for assessing the effect of congestion on emissions. Then from that framework, several simple and original equations are presented which can be used for sketch-level planning to estimate emissions impacts from congestion mitigation. Finally, application of the framework provides quantitative support for the decoupling of congestion and emissions mitigations. 


\section{ACKNOWLEDGMENTS}

Writing a master's thesis is a daunting task for any budding scholar. And while Stumptown and Sparks can get you far, you cannot reach completion without a supporting network of mentors, colleagues, loved ones, and biking buddies. I am deeply appreciative of my community of friends and scholars here in Portland who have buoyed me against the dragging anchor of this thesis.

My advisor, Dr. Miguel Figliozzi, provided invaluable feedback and support over many months of research. Many thanks also belong to the other two members of my thesis committee, Dr. Chris Monsere and Dr. Kelly Clifton, who in addition to providing feedback on this research taught me a great deal in my time at Portland State.

Furthermore, I am grateful for my previous advisor, Dr. Robert Bertini, who originally got me involved in transportation research and was the impetus for this thesis. Going back farther, I am indebted to Dr. Wendelin Mueller, my first research advisor, who propelled me along the research path and originally put the idea of graduate school into my thick head.

Along with my professors, I owe many thanks to the reviewers and colleagues who provided feedback on this research at its various stages. This includes my peers at Portland State University in engineering, planning, and environmental science. Lastly (but certainly not leastly) I want to express my humble gratitude to my invariably loving and patient family and friends, who give me more support and encouragement than I could hope to merit. You warm my heart. 
Vital material support for completion of my degree was provided by the U.S. Department of Transportation through the Eisenhower Graduate Transportation Fellowship, which granted a munificent stipend in addition to tuition and conference travel funding. Additional support for conference travel over the past two years came from the Oregon Transportation Research and Education Consortium (OTREC) and Portland State University. 


\section{TABLE OF CONTENTS}

ABSTRACT i

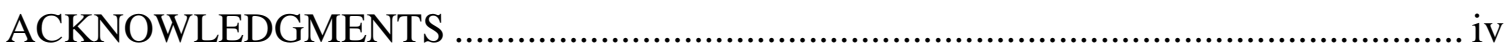

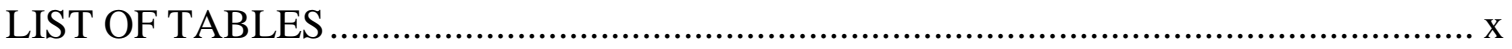

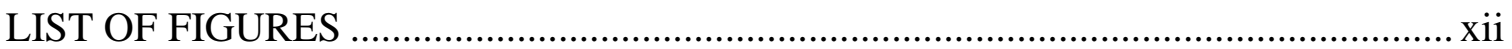

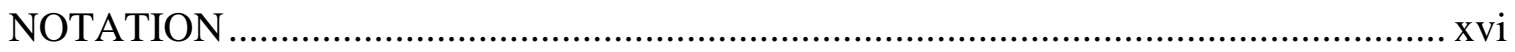

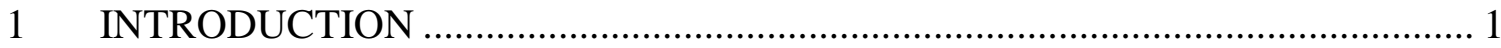

2 LITERATURE REVIEW AND BACKGROUND ............................................ 3

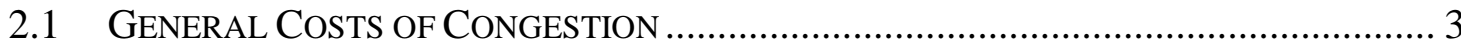

2.2 EMISSIONS FROM MOTOR VEHICLE TRAFFIC ...................................................... 7

2.3 METHODS FOR ESTIMATING CONGESTION IMPACTS ON EMISSIONS...................... 8

2.4 IMPACTS OF TRAFFIC CONGESTION ON VEHICLE EMISSIONS ............................... 14

2.5 TRAVEL DEMAND ELASTICITY .............................................................. 18

2.6 CongeSTION Mitigation AND EMISSIONS ..................................................... 22

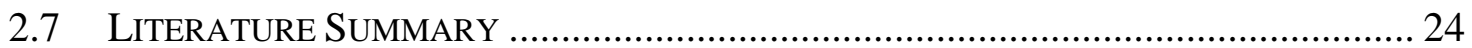

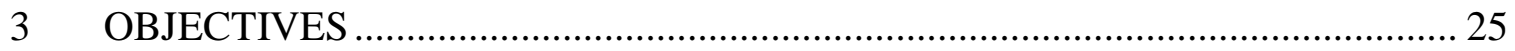

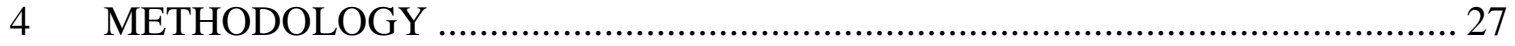

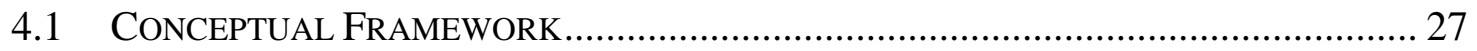

4.2 Macroscopic TrafFic Modeling ............................................................. 28 


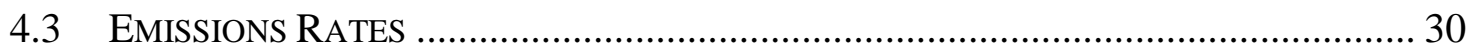

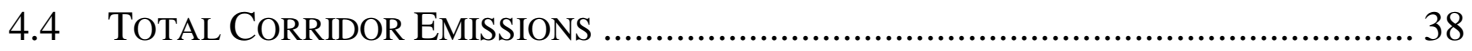

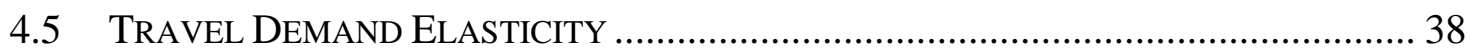

4.6 EMISSIONS GRADIENTS AND ELASTICITIES ........................................................ 41

4.7 Demand Elasticity For BREAK-Even EMISSIONS Conditions ......................... 45

4.8 OTHER DATA USED IN THE ANALYSIS ............................................................ 49

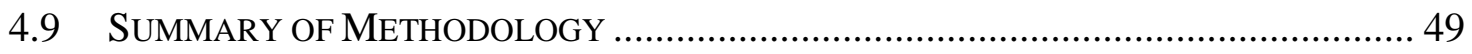

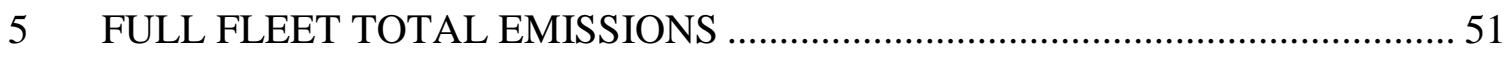

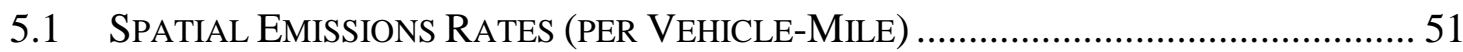

5.2 TEMPORAL EMISSIONS RATES (PER VEHICLE-HOUR) ......................................... 62

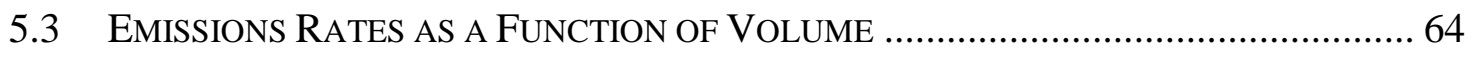

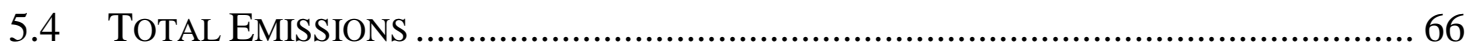

5.5 Total Emissions Elasticity to SPEED - InCLUding Variable Traffic

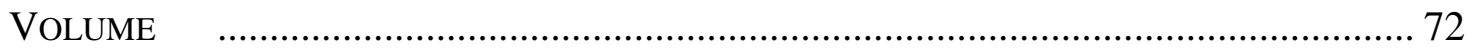

5.6 EMISSIONS BREAK-EvEN DEMAND ELASTICITY TO SPEED.................................. 75

5.7 Total Emissions Elasticity to Travel Demand Volume .............................. 81

6 THE IMPACTS OF ADVANCED VEHICLE TECHNOLOGIES ......................... 84

6.1 Emissions Rate Sensitivity to SPEed and AdVanced Vehicles ................... 84

6.2 Total Emissions Sensitivity to SPEed and AdVAnced Vehicles ................. 85

6.3 BREAK-EVEn Demand Elasticity AND AdVANCEd VehicLES .......................... 86

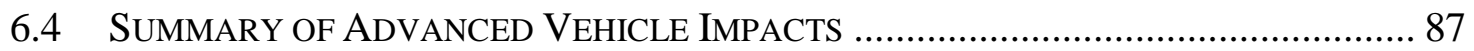


7.1 EMISSIONS RATES AND HD/LD Vehicle ClaSSES ......................................... 88

7.2 Total Emissions AND HD/LD Vehicle ClassES.......................................... 93

7.3 Total Emissions Elasticity CONSIDERING VARIABLE DEMAND AND HD/LD

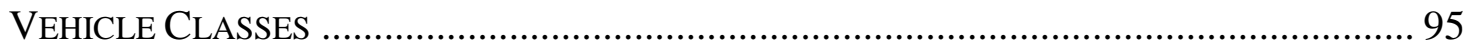

8 EMISSIONS IMPACTS OF CONGESTION MITIGATION ........................... 102

8.1 CAPACITY-Based Congestion Mitigation .................................................. 102

8.2 TRAVEL DEMAND REDUCTIONS .............................................................. 106

8.3 COMPARING STRATEGIES FOR EMISSIONS REDUCTIONS .................................. 107

9 VEHICLE CLASS-SEGREGATED FACILITIES ........................................ 122

9.1 TRUCK-ONLY LANE ANALYSIS ........................................................... 124

10 IMPLICATIONS FOR PERFORMANCE MEASURES ................................. 134

10.1 SpeEd-BASEd PERformance MeASURES.................................................... 134

10.2 Performance Measure Examples .......................................................... 136

10.3 Alternative Performance Measures ................................................... 141

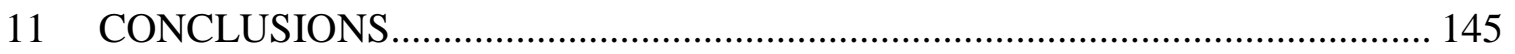

11.1 SKetch-Planning Equations For Elasticity OF EMISSIONS ..................... 145

11.2 EMISSIONS RATES ............................................................................. 146

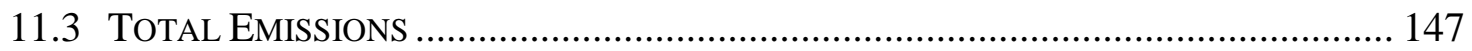

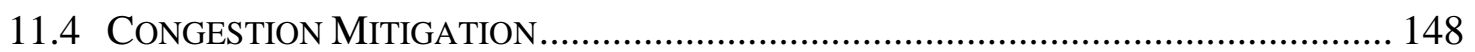


11.5 Final Thoughts And FutUre Work .................................................... 150

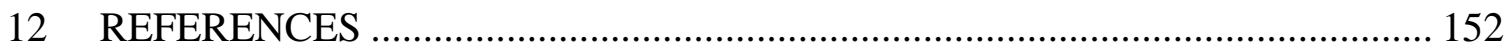

APPENDIX A: MAJOR STUDIES OF THE IMPACTS OF CONGESTION ON

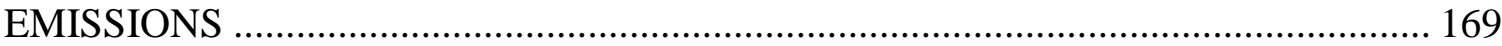




\section{LIST OF TABLES}

Table 1: Summary of Roadway Congestion Effects on Vehicle Emissions ..................... 16

Table 2. Elasticity of Travel Demand (Distance) to Travel Speed from the Literature.... 20

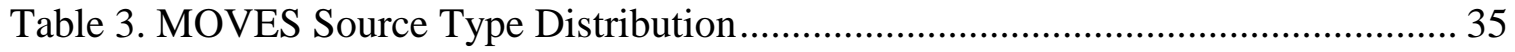

Table 4. Full-Fleet MOVES Emissions-Speed Curve Fit Parameters for Freeways ........ 51

Table 5. Full-Fleet MOVES Emissions-Speed Curve Fit Parameters for Arterials ......... 57

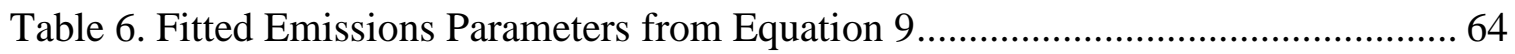

Table 7. MOVES Emissions-Speed Curve Fit Parameters for $e_{l}$ on Freeways ................ 88

Table 8. MOVES Emissions-Speed Curve Fit Parameters for $e_{h}$ on Freeways ............... 88

Table 9. Comparison of Equivalent Emissions Reduction Strategies for Freeway $\mathrm{CO}_{2} \mathrm{e}$ Emissions (MOVES Model with Inelastic Demand, $\left.\varepsilon_{q}^{v}=0\right) \ldots \ldots \ldots \ldots \ldots \ldots \ldots \ldots \ldots . \ldots \ldots 111$

Table 10. Comparison of Equivalent Emissions Reduction Strategies for freeway $\mathrm{CO}_{2} \mathrm{e}$

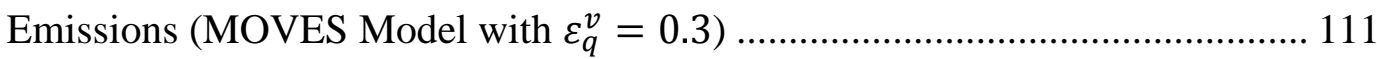

Table 11. Comparison of Equivalent Emissions Reduction Strategies for Arterial $\mathrm{CO}_{2} \mathrm{e}$

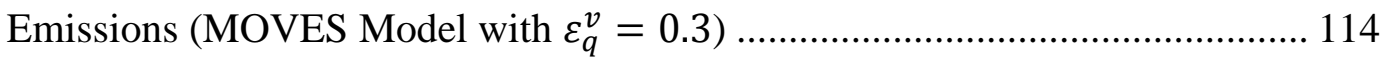

Table 12. Comparison of Equivalent Emissions Reduction Strategies for Freeway $\mathrm{NO}_{\mathrm{x}}$

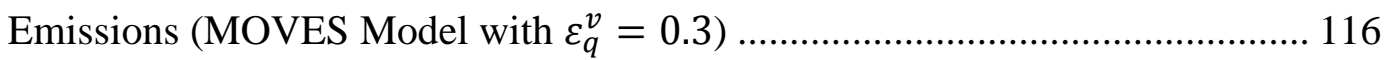

Table 13. Comparison of Equivalent Emissions Reduction Strategies for Arterial $\mathrm{NO}_{\mathrm{x}}$ Emissions (MOVES Model with $\varepsilon_{q}^{v}=0.3$ ) 116

Table 14. Comparison of Equivalent Emissions Reduction Strategies for Freeway $\mathrm{PM}_{2.5}$ Emissions (MOVES Model with $\varepsilon_{q}^{v}=0.3$ ) 117 
Table 15. Comparison of Equivalent Emissions Reduction Strategies for Arterial $\mathrm{PM}_{2.5}$ Emissions (MOVES Model with $\varepsilon_{q}^{v}=0.3$ ) 117

Table 16. Summary of Percent Change in Emissions on Freeways from CBS (MOVES-

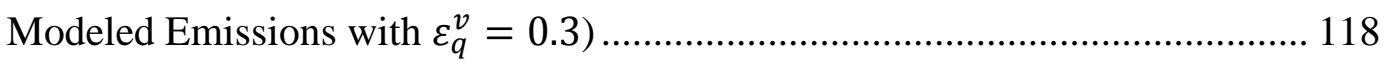

Table 17. Summary of Percent Change in Emissions on Arterials from CBS (MOVES-

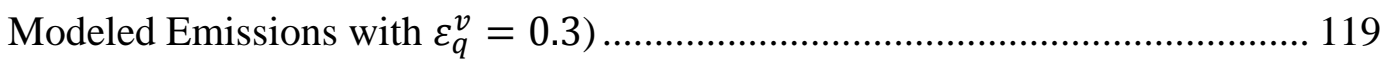

Table 18. Congestion \& Emissions Mitigation Strategies: Direct Impacts ................... 123

Table 19. Volume, Speed, and $\mathrm{CO}_{2}$ e Emissions Changes with Lane Strategies ............ 127

Table 20. TTI and Total Emissions Effects of Lane Strategies Described in Section 9.1 


\section{LIST OF FIGURES}

Figure 1. Congestion-Emissions Influence Paths ....................................................... 25

Figure 2. Full-Fleet Freeway Emissions Rates versus Average Speed ( $e$ vs. $v$ ), with LOS

Figure 3. Full-Fleet Freeway Emissions Rate Gradients versus Average Speed, with LOS

Figure 4. Distribution of VMT using 5-minute average speeds from dual-loop detectors

on I-5 northbound in Portland, Oregon........................................................... 56

Figure 5. ESC and ESC Gradients for Arterial Emissions........................................ 58

Figure 6. Comparison of freeway and arterial emissions-speed gradients ..................... 59

Figure 7. Comparison of Speed and Acceleration Distributions for Freeway and Arterial

Drive Schedules with Similar Average Speeds ............................................. 61

Figure 8. Fleet $\mathrm{CO}_{2} \mathrm{e}$ emissions rates per mile and per minute, with freeway LOS ........ 63

Figure 9. $\mathrm{CO}_{2}$ Emissions Fits for MOVES and Barth Models by Equation 9................ 66

Figure 10. Total Emissions $(E)$ as Shading on the Speed $(v)$ versus Effective Demand

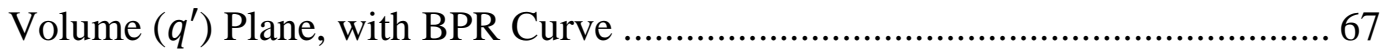

Figure 11. Total Emissions $(E)$ as Shading and Contours on the Vehicle Speed $(v)$ versus

Vehicle Throughput (q) Plane, with Observed Traffic States ........................... 69

Figure 12. Elasticity of Emissions Rates to Speed, $\varepsilon_{e}^{v}$, by MOVES Model ................... 71

Figure 13. Elasticity of Total $\mathrm{CO}_{2} \mathrm{e}$ Emissions to Changes in Speed, $\varepsilon_{E}^{v}$, as Shadings and

Contours on the $\eta_{q}^{v}$ versus $v$ Plane. 73 
Figure 14 Elasticity of Total Emissions to Speed, $\varepsilon_{E}^{v}$, for CO, NOx, PM2.5, and HC, as Shadings and Contours on the $\eta_{q}^{v}$ versus $v$ Plane .......................................... 74

Figure 15. The Effect of a Capacity Increase on Total Emissions Depends on the Final Demand Volume; Shading is Total Freeway $\mathrm{CO}_{2} \mathrm{e}$ Emissions Rate, $E$, by the MOVES Model 76

Figure 16. Calculated Emissions Break-Even Elasticities of Travel Demand to Travel

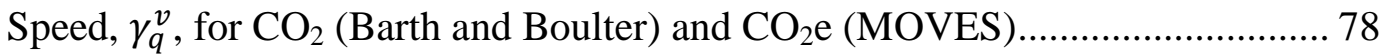

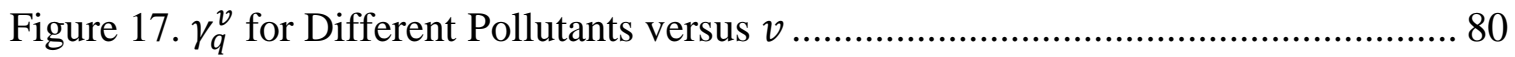

Figure 18. Elasticity of Total $\mathrm{CO}_{2}$ e Emissions to Travel Demand Volume, $\varepsilon_{E}^{q}$, along with $\varepsilon_{e}^{v}$ and $\varepsilon_{v}^{q}$ 82

Figure 19. ${ }^{e_{h}} / e_{l}$ versus $v$ 89

Figure 20. Diesel/Gasoline Vehicle Emissions Rate Ratios for Passenger Trucks (left) and

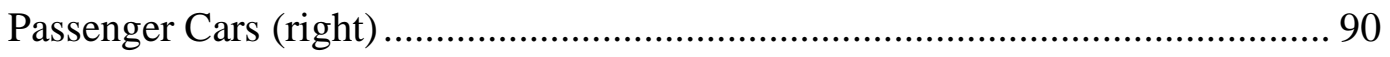

Figure 21 (Part II). Fleet Emissions Rate Sensitivity to $f_{h}$, with freeway LOS 93

Figure 22. Comparison of Total Emissions from Mixed and LD-only Fleets, adjusted for PCE, as $E / E_{\text {all-LD }}$ versus $v$ 94

Figure 23. Vehicle Class-Specific Emissions Break-Even Elasticities for $\mathrm{CO}_{2}, \gamma_{q_{l}}^{v_{l}}$ and $\gamma_{q_{h}}^{v_{h}}$ versus $v$ 95

Figure 24. Vehicle Class-Specific Emissions Break-Even Elasticities for Other Pollutants, $\gamma_{q_{l}}^{v_{l}}$ and $\gamma_{q_{h}}^{v_{h}}$ versus $v$ 96 
Figure 25. Fraction of Total Emissions from HD Vehicles, $E_{h} / E$, versus $v\left(f_{h}=0.1\right) .98$

Figure 26. $\mathrm{CO}_{2}$ e Emissions Elasticity to Average Speed, $\varepsilon_{E}^{v}$, with $f_{h}=0.1$ and $\eta_{q_{h}}^{v_{h}}$ at (a) $100 \%$, (b) $50 \%$, and (c) $0 \%$ of $\eta_{q_{l}}^{v_{l}}$ 100

Figure 27. Characterization of CBS Based on Ranges of Break-Even Elasticities, $\gamma_{q_{j}}^{v_{j}} .104$

Figure 28. Characterization of CBS Based on Ranges of Break-Even Elasticities for LD

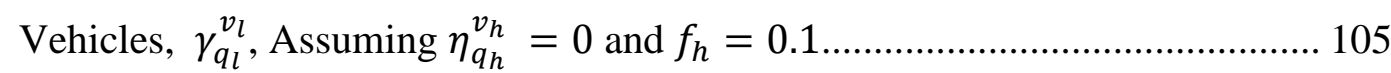

Figure 29. Emissions Elasticity to EV Penetration, $\varepsilon_{E}^{f_{e}}$, versus $e_{h} / e_{l}$, assuming $f_{e}=0$ and $e_{e}=0$

Figure 30. Percent Reductions in Total Emissions for Each Land Strategy and Pollutant 128

Figure 31. Effect of Lane Strategies on Total $\mathrm{CO}_{2} \mathrm{e}$ Emissions with Varying Initial $q$ (Vehicle Travel Demand for 3 GP Lanes); Other Assumptions as Above 129

Figure 32. Effect of Lane Strategies on Total $\mathrm{CO}_{2} \mathrm{e}$ Emissions with Varying Initial $f_{H}$;

Other Assumptions as Above.

Figure 33. Effect of Lane Strategies on Total $\mathrm{CO}_{2} \mathrm{e}$ Emissions with Varying Demand Elasticity $\eta_{q_{j}}^{v_{j}}$ (for Both LD and HD Vehicles); Other Assumptions as Above.. 131

Figure 34. Effect of Lane Strategies on Total $\mathrm{CO}_{2} \mathrm{e}$ Emissions with Varying Demand Elasticity for HD Vehicles; Other Assumptions as Above 132

Figure 35. Daily Peak-Period $\mathrm{CO}_{2} \mathrm{e}$ Emissions per Peak-Period Traveler versus TTI, Segmented by Urban Area Population Size................................................... 139 
Figure 36. Daily Peak-Period $\mathrm{CO}_{2} \mathrm{e}$ Emissions per Peak-Period Traveler versus PeakPeriod VMT per Peak-Period Traveler, Segmented by Urban Area Population Size

Figure 37. Total Daily Peak-Period $\mathrm{CO}_{2} \mathrm{e}$ Emissions versus TTI, Segmented by Urban Area Population Size......................................................................... 141

Figure 38. Daily Peak-Period $\mathrm{CO}_{2} \mathrm{e}$ Emissions per Peak-Period Traveler versus PeakPeriod VHT per Peak-Period Traveler, Segmented by Urban Area Population Size

Figure 39. Changes in Performance per Peak-Period Traveler for Portland, Oregon, from 1982 to 2009 143

Figure 40. Comparison of Changes in TTI, Travel Time per Peak-Period Traveler, and VMT per Peak-Period Traveler versus Changes in Total Emissions per PeakPeriod Traveler between 1999 to 2009 for all Urban Areas in the UMR 144 


\section{NOTATION}

\section{TRAFFIC VARIABLES}

- $L \quad$ length of a section of roadway under consideration (distance)

- $\bar{t} \quad$ average travel rate over a section of roadway for all vehicles (time/distance)

- $t_{0}$ average travel rate over a section of roadway in free-flow conditions (time/distance)

- $t_{j} \quad$ average travel rate over a section of roadway for vehicles of class $j$ (time/distance)

- $\bar{v} \quad$ average travel speed over a section of roadway for all vehicles (distance/time)

- $v_{j} \quad$ average travel speed over a section of roadway for vehicles of class $j$ (distance/time)

- $v_{0} \quad$ average travel speed over a section of roadway in free-flow conditions (distance/time)

- $q$ total volume rate of vehicles traversing a section of roadway (vehicles/time)

- $q_{j} \quad$ volume rate of vehicles of vehicle class $j$ traversing a section of roadway (vehicles/time) 
- $q^{\prime} \quad$ effective flow: volume rate of vehicles in passenger-car equivalent units traversing a section of roadway, per lane (passenger-cars/lane/time)

- $\hat{q} \quad$ actual vehicles throughput at a location on a section of roadway (vehicles/time)

- $V M T_{j} \quad$ travel demand distance of vehicles of vehicle class $j$ traversing a section of roadway (vehicles-miles/time)

- $c$ effective capacity: vehicle carrying capacity of the roadway in passenger-cars per lane (passenger-cars/lane/time)

- $\alpha$ dimensionless parameter for the Bureau of Public Roads (BPR) volumetravel time function

- $\beta$ dimensionless parameter for the BPR function

\section{FLEET VARIABLES}

- $j \quad$ vehicle class in the set of vehicle classes $J$

- $J \quad$ set of mutually exclusive and exhaustive vehicle classes

- $s \quad$ emissions source type used in emissions modeling

- $S \quad$ set of all emissions source types modeled

- $S_{j} \quad$ set of emissions source types contained in vehicle class $j$

- $f_{j} \quad$ fractional fleet composition (by distance traveled) of vehicle class $j$

- $f_{s}$ fractional fleet composition (by distance traveled) of emissions source type $s$ 
- $P C E_{j} \quad$ passenger car equivalents for vehicle class $j$

\section{EMISSIONS VARIABLES}

- $e_{s} \quad$ spatial marginal emissions rate from emissions source type $s$ (mass/vehicle-distance)

- $e_{j} \quad$ average spatial marginal emissions rate from vehicles of class $j$ (mass/vehicle-distance)

- $\bar{e} \quad$ average spatial marginal emissions rate from all vehicles (mass/vehicledistance)

- $\bar{e}^{\prime} \quad$ average temporal marginal emissions rate from all vehicles (mass/vehicle-time)

- $a_{i, j} \quad$ parameters from emissions rate equation for $e_{j}\left(v_{j}\right)$, for vehicle class $j$

- $a_{i} \quad$ parameters from emissions rate equation for $\bar{e}(\bar{v})$, for all vehicles

- $n$ power parameter from emissions rate equations for $e_{j}\left(v_{j}\right)$ and $\bar{e}(\bar{v})$

- $b_{0}, b_{1}, b_{2}, m$ parameters from emissions rate equation for $\bar{e}\left(q^{\prime}\right)$

- E total emissions from all on-road vehicles (mass/time/road-distance)

- $E_{j}$ total emissions from on-road vehicles of class $j$ (mass/time/roaddistance)

\section{ELASTICITIES}

- $\eta_{q_{j}}^{t_{j}} \quad$ elasticity of $q_{j}$ to $t_{j}$ for vehicle class $j$ 
- $\eta_{q_{j}}^{v_{j}} \quad$ elasticity of $q_{j}$ to $v_{j}$ for vehicle class $j$

- $\eta_{q}^{\bar{v}} \quad$ aggregate elasticity of $q$ to $\bar{v}$ for all vehicles

- $\quad \eta_{V M T_{j}}^{v_{j}} \quad$ elasticity of $V M T_{j}$ to $v_{j}$ for vehicle class $j$

- $\varepsilon_{e_{j}}^{v_{j}} \quad$ elasticity of $e_{j}$ to $v_{j}$ for vehicle class $j$

- $\varepsilon_{E_{j}}^{v_{j}} \quad$ elasticity of $E_{j}$ to $v_{j}$ for vehicle class $j$

- $\varepsilon_{E}^{\bar{v}} \quad$ elasticity of $E$ to $\bar{v}$ for all on-road vehicles

- $\varepsilon_{v_{j}}^{q_{j}} \quad$ elasticity of $v_{j}$ to $q_{j}$ for vehicle class $j$

- $\varepsilon_{\bar{v}}^{q} \quad$ elasticity of $\bar{v}$ to $q$ for all on-road vehicles

- $\varepsilon_{e_{j}}^{q_{j}} \quad$ elasticity of $e_{j}$ to $q_{j}$ for vehicle class $j$

- $\varepsilon_{\bar{e}}^{q} \quad$ elasticity of $\bar{e}$ to $q$ for all on-road vehicles

- $\quad \varepsilon_{E_{j}}^{q_{j}} \quad$ elasticity of $E_{j}$ to $q_{j}$ for vehicle class $j$

- $\varepsilon_{E}^{q} \quad$ elasticity of $E$ to $q$ for all on-road vehicles

- $\varepsilon_{E}^{T T I}$ elasticity of $E$ to TTI for all on-road vehicles

- $\gamma_{q_{j}}^{v_{j}} \quad$ elasticity of $q_{j}$ to $v_{j}$ for vehicle class $j$ which leads to emissions breakeven conditions

- $\gamma_{q}^{\bar{v}} \quad$ elasticity of $q$ to $\bar{v}$ for all vehicles which leads to emissions break-even conditions 


\section{INTRODUCTION}

Motorized transportation's role in decreasing urban air quality and increasing atmospheric greenhouse gases through motor vehicle emissions is a global concern (Fenger, 1999; U.S. Environmental Protection Agency, 2009). Concurrently, roadway congestion impacts urban areas throughout the world with varying economic, social, and environmental costs (European Conference of Ministers of Transport (ECMT), 2007; HDR, 2009). But the full effects of traffic congestion on motor vehicle emissions are still not well quantified because of interactions and impacts on many scales, from vehicle maintenance to land use.

Policy-makers, researchers, and activists often assume that congestion reductions inevitably lead to reduced vehicle emissions. In many cases, emissions reductions are cited as an implicit benefit of congestion mitigation without proper justification or quantification of the benefits. For example, the U.S. Federal Highway Administration's Congestion Mitigation and Air Quality (CMAQ) Improvement Program suggests a clear co-beneficial relationship between the two. The CMAQ program has provided over $\$ 14$ billion in funding since 1991 for transportation projects to improve air quality and reduce congestion (Federal Highway Administration, 2010) - one third of it for traffic flow improvement projects (Grant et al., 2008; Transportation Research Board, 2002).

If congestion mitigation is to be tied to air quality goals, we need better understanding of total congestion impacts on motor vehicle emissions. Toward that goal, this thesis presents a unique conceptual and mathematical framework for assessing the 
effects of congestion on emissions (Chapter 4). This framework includes the influences of varying travel efficiency and travel volume in a parsimonious way. From this framework, results, equations, and insights are presented which can be used for sketchlevel planning to estimate emissions impacts of congestion mitigation (Chapter 5).

Beyond aggregate full-fleet emissions, we investigate the impacts of advanceddrivetrain vehicles (Chapter 6) and heavy-duty vehicles (Chapter 7) on the congestionemissions relationship. We then apply the sketch-planning approach to investigate conditions in which emissions co-benefits can broadly be expected from capacity-based congestion mitigation, including a comparison of alternative emissions reduction strategies (Chapter 8). Further analysis presented in this thesis includes the emissions impacts of vehicle class-segregated road facilities (Chapter 9) and the emissions implications of congestion performance measures (Chapter 10). The results in this thesis provide quantitative support for the decoupling of congestion and emissions mitigations. A literature review with background information and research objectives are presented next in Chapters 2 and 3, respectively. 


\section{LITERATURE REVIEW AND BACKGROUND}

The broad extent of congestion on urban roadway networks is well documented in the literature (ECMT, 2007; Schrank \& Lomax, 2007). Not only are nearly all major metropolitan areas in the U.S. congested during peak periods, but congestion is increasing with population growth and urban densification. This growth in congestion is magnified in developing countries as the rate of automobile ownership and usage is increasing as well. Although there is no debate that congestion exists and is growing, the full impacts of congestion (and how best to address them) are not yet decided. In this chapter we describe the state of knowledge about the relationship between traffic congestion and motor vehicle emissions.

\subsection{General Costs of Congestion}

Various studies have attempted to quantify the impacts of congestion (Goodwin, 2004; HDR, 2009; Kriger, C. Miller, Baker, \& Joubert, 2007; Weisbrod, Vary, \& Treyz, 2001). The suggested impacts include direct effects such as excess travel time and indirect effects such as increased business operating costs and human exposure to pollution. Across multi-dimensional studies, excess travel time is consistently the largest estimated social cost of congestion (HDR, 2009; Kriger et al., 2007; Schrank, Lomax, \& Turner, 2010). But comprehensive attempts to quantify total congestion impacts suffer from challenges such as estimating the extent of higher-order, indirect effects (e.g. congestion impacts on land use) and quantifying intangibles (e.g. traveler stress levels). 
Too often, congestion cost analyses do not even go as far as to estimate driver behavior responses to congestion (such as mode shift).

\subsubsection{Congestion Benchmarks}

Congestion studies are also inhibited by inconsistent definitions and thresholds of congestion. A 'congestion-free' scenario is typically used as a benchmark for estimating congestion effects, but the attributes of this hypothetical situation are not manifest. Probably the most common benchmarking approach is to simply compare congested speeds to free-flow or uncongested threshold speeds (Greenwood, Dunn, \& Raine, 2007; HDR, 2009; Kriger et al., 2007; Schrank \& Lomax, 2009). The hypothetical system change, then, is limitless roadway supply, with all existing travel demand serviced without impedance. The problem with this approach is that it ignores suppressed demand from exiting congestion - an effect described by Hymel, Small \& Dender (2010). Ignoring this suppressed or latent travel demand distorts the costs of congestion and can potentially magnify the predicted benefits of congestion mitigation.

The European Conference of Ministers of Transport (ECMT) criticizes a freeflow speed benchmark as suggestive of unattainable and unaffordable policy outcomes (2007). Goodwin (2004) also provides a sound critique of the fixed-demand, free-flow comparison approach to congestion cost estimates. He points out that because of induced demand, free-flow conditions with existing traffic volumes are unrealistic: "a purely notional idea, not a conceivable description of a world we might choose to provide for." Hence, congestion indicators and cost estimates need more clear and consistent 
benchmarking to be comparable and realistic: benchmarks that fully represent an alternative situation to the congested roadway.

\subsubsection{Congestion Performance Measures}

As with cost estimates, congestion performance measures are also saddled with the distortions of fixed-demand/free-flow speed comparisons. Most congestion performance indicators measure mobility or its impedance (Cambridge Systematics, Inc. $\&$ Texas Transportation Institute, 2005). Mobility is typically estimated as average travel speed or its inverse (distance-normalized travel time) - which neglects changes in travel distances and travel volume. This approach has the same problems described above for fixed-demand congestion cost estimates, which are unrealistic and misleading. Cortright (2010) criticizes the approach of the Texas Transportation Institute's Urban Mobility Report (Schrank et al., 2010), which uses a normalized travel time metric for its primary congestion indicator. Cortright describes this as an unrealistic measure which neglects the roles of travel distances, land use, sprawl and accessibility.

\subsubsection{Recurring and Non-recurring congestion}

The transportation literature often distinguishes between recurring and nonrecurring congestion. Recurring congestion is essentially the expected, daily delay connected to peak travel demand. Nonrecurring congestion is unexpected or unusual congestion caused by incidents such as crashes, inclement weather, special events, debris, roadside distractions, and even announced construction closures (Dowling, Skabardonis, Carroll, \& Wang, 2004; Kwon, Mauch, \& Varaiya, 2006; Skabardonis, Varaiya, \& Petty, 
2003). The causal distribution of nonrecurring congestion is location-specific (Dowling et al., 2004), and the nonrecurring portional contribution to total congestion has been reported in ranges from 13-70\% (Kwon et al., 2006; Skabardonis et al., 2003; Hallenbeck, Ishimaru, \& Nee, 2003).

To the author's knowledge, no published studies have revealed an intrinsic difference in microscopic traffic flow characteristics between recurring and nonrecurring congestion for the same roadway and delay characteristics (though nonrecurring congestion is often caused by a sudden, temporary change in capacity). Differences in road users' expectancy could lead to different trip-level behavior responses to recurring and non- recurring congestion (such as departure time choice and routing), since non- recurring congestion is harder to anticipate.

Non- recurring congestion decreases travel time reliability - which is valued by travelers and so likely to influence their travel behavior (Brownstone \& Small, 2005; Small, Winston, \& Yan, 2005). Some travel time unreliability, though, is also associated with fluctuating/uncertain travel demand and roadway capacity $(\mathrm{Tu}$, van Lint, \& van Zuylen, 2007), such as would be encountered during peak period recurring congestion. Bigazzi and Figliozzi (2011) showed that the instability in traffic flows near roadway capacity increases time, fuel, and emissions costs for freeway travel, though high flow/capacity ratios can occur during recurring or non- recurring congestion. The differing cost implications for recurring and non- recurring congestion is an area needing research, particularly as it relates to emissions. 


\subsection{Emissions from Motor Vehicle Traffic}

One of the external costs cited by congestion studies is increased emissions of air pollutants from motor vehicles. This claim is explored more in the literature review below, and is the subject of this thesis. Motor vehicle emissions in general are a significant contributor to poor air quality in urban areas (Fenger, 1999), with large health effects (Health Effects Institute, 2010). According to data from the U.S. Environmental Protection Agency (EPA), "Highway Vehicles" was the single source category with the largest emissions of carbon monoxide $(\mathrm{CO})$, nitrogen oxides $\left(\mathrm{NO}_{\mathrm{x}}\right)$, and volatile organic compounds (VOC) in the U.S. in 2002 (U.S. Environmental Protection Agency, n.d.). On-road vehicles also emit roughly one quarter of greenhouse gas emissions in the U.S. (U.S. Environmental Protection Agency, 2009).

Vehicle emissions of local pollutants and greenhouse gases have different scales of impacts, temporally and spatially. Local pollutants such as $\mathrm{CO}, \mathrm{VOC}, \mathrm{NO}_{\mathrm{x}}$, and particulate matter (PM) can be relatively short-lived and generally only impact the region or even street where they are emitted. Health research has suggested a 50 to 1,500 meter impact zone from highways and major roads for measured health impacts from local pollutants (Health Effects Institute, 2010). Greenhouse gas emissions such as carbon dioxide $\left(\mathrm{CO}_{2}\right)$, on the other hand, have minimal local impact but are important in terms of cumulative global emissions, and over long time scales. This delineation is a generalization, as some pollutants have an immediate local impact and also contribute to climate change (sometimes after atmospheric transformation). Also, some pollutants 
impact air quality primarily through formation of harmful secondary pollutants such as ozone and acid rain - which delays their temporal influence and broadens their spatial influence.

The primary factors influencing on-road vehicle emissions are the quantity of vehicle travel (typically assessed as vehicle-miles traveled, or VMT), the vehicle engine loads (often characterized by operating modes or speed profiles), and the vehicle fleet characteristics (vehicle type, condition, emissions technology, etc.) (Kuhlwein \& Friedrich, 2000, 2005; Pandian, Gokhale, \& Ghoshal, 2009; Singh, Huber, \& Braddock, 2007). These factors, therefore, are of principal interest in examining congestion effects for possible emissions implications. Additionally, not all pollutants react the same way to changing vehicle/road conditions (Barth, Scora, \& Younglove, 1999; Boulter, Barlow, McCrae, \& Latham, 2009), so different pollutants should be considered separately. In general, more research is needed on the correlations of emissions rates of different pollutants under various traffic/fleet conditions - especially between greenhouse gases and local air pollutants - which would allow more generalization of congestion effects.

\subsection{Methods for Estimating Congestion Impacts on Emissions}

Before describing the body of research results relating congestion to emissions, it is worthwhile to look at how congestion-emissions relationships have been studied. Despite increasing research interest, the wide breadth of congestion effects continues to hinder comprehensive investigations of congestion impacts on emissions. It is nearly impossible to simultaneously trace/model all potential connecting pathways from 
congestion to emissions, so studies are forced to draw effect boundaries fit to the resolution and scope of available data and models. Existing research thus typically measures the emissions effects of specific congestion characteristics through select influence paths.

\subsubsection{Empirical Emissions Quantification}

After establishing an uncongested benchmark scenario, emissions during congested and uncongested traffic conditions can be compared directly through emissions measurements. This turns out to be a difficult approach, though, because of necessarily limited sampling. Ropkins et al. (2009) provide a detailed description of the qualities of emissions monitoring techniques relating to factors captured and spatial coverage.

Individual vehicle emissions can be measured on-road using probe vehicles (Barth, Scora, \& Younglove, 2004; Holmen, Sentoff, Robinson, \& Montane, 2010) or in a laboratory using realistic driving speed patterns for vehicles on a chassis dynamometer (Barth et al., 2000; Smit, Smokers, \& Rabe, 2007). These methods measure true vehicle emissions and avoid some of the challenges of other approaches, but their relevance depends on the representativeness of the vehicles sampled and driving patterns applied. It is also a costly approach if broad samples of vehicles and traffic conditions are desired.

In-situ measurement of roadway air quality can capture full, representative emissions or air quality effects, but experimental factors are difficult to control and emissions effects can only be quantified for existing roadway conditions (Ropkins et al., 2009). Most often, roadside air quality measurements are gathered concurrently with 
traffic data to establish empirical relationships between the two (Bigazzi, Van Lint, Klunder, Stelwagen, \& Ligterink, 2010; H. Chen, Namdeo, \& Bell, 2008; Kohler, Corsmeier, Vogt, \& Vogel, 2005). A major challenge of using pollution concentration measurements to estimate on-road vehicle emissions is controlling for dispersion influences, which creates large uncertainties (Venkatram, 2004; Venkatram, Isakov, Thoma, \& Baldauf, 2007). Additionally, in-situ measurements are generally quite limited in spatial extent and so subject to confounding factors related to the measurement location (Croxford \& Penn, 1998).

\subsubsection{Emissions Modeling}

As an alternative to direct measurement of emissions or air quality, emissions modeling is more often employed because it allows full control over study factors and estimation of emissions in hypothetical scenarios (useful for mitigation planning). Additionally, modeling can estimate a wider range of vehicle/traffic conditions given limited resources. The broad typical approach to emissions modeling is to estimate an average emissions rate per vehicle-mile of travel (per VMT), and then combine the emissions rate with estimates of the volume of vehicle travel, VMT.

The main drawbacks of emissions modeling are high uncertainty (Kuhlwein \& Friedrich, 2000; Frey \& Zheng, 2002; Joumard, Philippe, \& Vidon, 1999), lack of standards (Adler, Grant, \& Schroeer, 1998), difficulty in validating (Dowling, 2005), and challenges in modeling on multiple scales. The uncertainty in emissions models is context-dependent and not necessarily prohibitive (Frey \& Zheng, 2002), though the 
effects can be magnified if emissions are modeled in series with traffic and/or dispersion models - e.g. (J. Y. Park, Noland, \& Polak, 2001). Because of limited calibration data, emissions models have known accuracy weaknesses related to heavy-duty vehicles and particulate emissions (Dowling, 2005) - though these are being addressed with newer models (Barth et al., 2004; U.S. Environmental Protection Agency, 2009a).

\subsubsection{Microscopic Emissions Modeling}

Microscopic modeling combines detailed traffic data with a microscopic emissions model to investigate the effects of changes in detailed traffic characteristics (Barth et al., 1999; Rakha, Van Aerde, Ahn, \& Trani, 2000). This level of detail can be important because short, intense accelerations can produce emissions rates hundreds of times higher than 'normal' driving (Joumard et al., 1999). As an example of where this type of modeling is needed, small roadway changes such as continuous-access versus limited-access High-Occupancy Vehicle (HOV) lanes can significantly impact emissions because of increased weaving intensity (Boriboonsomsin \& Barth, 2008).

The significant data demands of microscopic emissions models are difficult to satisfy (Pandian et al., 2009). Most models require second-by-second vehicle speed data, which can be obtained from probe vehicles (Malcolm, Younglove, Barth, \& Davis, 2003) or traffic micro-simulation (K. Chen \& Yu, 2007; Hirschmann \& Fellendorf, 2010). Driving data based on probe vehicles, like the empirical emissions estimation methods described above, limit the study to existing traffic conditions. Microscopic traffic simulation models can create various congestion scenarios, but they have not been well 
validated for use in creating inputs to microscopic emissions models (Dowling, 2005; J. Y. Park et al., 2001) - primarily because of unrealistic accelerations (Hirschmann \& Fellendorf, 2010) and speed fluctuations (Jackson \& Aultman-Hall, 2010). Other challenging data requirements are detailed vehicle information, meteorology data, and fuel data. Finally, while microscopic modeling can replicate the effects of detailed congested traffic flow characteristics, larger network and behavioral effects are difficult to include because of limited spatial/temporal coverage (Dowling, 2005).

\subsubsection{Macroscopic Emissions Modeling}

Most macroscopic emissions models use average travel speed as the primary traffic input (Barlow \& Boulter, 2009; Smit et al., 2007; U.S. Environmental Protection Agency, 2009a). As with microscopic emissions modeling, relevant vehicle activity can be collected on-road but is often modeled instead (see Ziesman \& Rilett (2001) for a comparison). Average speed inputs allow macroscopic emissions models to be easily interfaced with travel demand models for regional emissions estimates (Anderson, Kanaroglou, E. Miller, \& Buliung, 1996; Affum, Brown, \& Chan, 2003; Roberts, Washington, \& Leonard II, 1999). However, research has shown that travel demand model outputs require additional post-processing before use in macroscopic emissions modeling (Bai, Nie, \& Niemeier, 2007).

A typical method for using average speeds to estimate emissions is by assuming an archetypal driving pattern that matches an average speed (these are usually represented by $1 \mathrm{~Hz}$ vehicle speed time-series, also called drive cycles, speed profiles, or drive 
schedules, depending on the application) (Lin \& Niemeier, 2003a; U.S. Environmental Protection Agency, 2009a). For more accuracy, these drive cycles can be specific to the relevant vehicle type, roadway facility type, and even region of the country (Lin \& Niemeier, 2003b). There are other methods of macroscopic emissions modeling (such as multilinear regression), but these still assume a representative pattern of driving at some point in the data collection or model development.

Average-speed emissions models can capture typical characteristics of roadway congestion if the imbedded drive cycles are facility-specific and sufficiently representative (Smit, Brown, \& Chan, 2008). Fortunately, the applicability of drive cycles for "real-world" driving conditions has received considerable attention by researchers (Ericsson, 2000; Joumard et al., 1999; Lin \& Niemeier, 2003b, 2002; Nesamani \& Subramanian, 2006). Since macroscopic emissions models are not applicable for "nonstandard" driving (Frey \& Zheng, 2002) atypical traffic features or traffic management strategies must be modeled with caution.

Although macroscopic modeling neglects unique, detailed traffic characteristics, it is better suited than microscopic models to measure the indirect, broader influences of congestion. Dowling (2005) suggests a hybrid modeling methodology to address the congestion-emissions question because of the infeasibility of microscopic modeling to capture demand effects and limitations of macroscopic modeling to detect more subtle operational benefits. 


\subsubsection{Congestion Benchmarks}

One final note on emissions estimation methodologies relates to the congestion thresholds and cost comparisons discussed above. Uncongested comparison conditions for emissions estimates can be characterized from real-world free-flow traffic or simulated as constant-speed steady-state traffic flow. Hypothetical constant-speed driving generates lower emissions rates than real-world driving around free-flow speeds, which has intrinsic variability (Barth \& Boriboonsomsin, 2008; Barth et al., 1999; Jackson \& Aultman-Hall, 2010). Hence, congestion indicators and cost estimates should use realistic transient free-flow speed profiles (not steady-state speeds) for comparisons. Ideally, congestion studies should further account for uncongested differences in the vehicle fleet, though this is rarely done.

\subsection{Impacts of Traffic Congestion on Vehicle Emissions}

When they are included in total congestion cost estimates, emissions are typically a very small portion of total costs (HDR, 2009; Kriger et al., 2007). These results, though, are subject to the uncertainty of applying an economic-equivalent value to an externality such as pollution emissions (see Hall, Brajer, \& Lurmann (2008) for an example of the complexity in valuing air pollution). Furthermore, to the author's knowledge no comprehensive congestion cost study has estimated costs with a benchmark other than the fixed-demand/free-flow speed approach - with all its limitations described above. In other words, the broad cost studies have all ignored the effects of variable demand on emissions. 
The major published studies focusing on the congestion-emissions relationship are summarized individually in Appendix A. The general consensus is that the total emissions effects of congestion are either not well understood or highly variable. A recent analysis for the U.S. Department of Transportation (HDR, 2009) asserts that the total impact of congestion on emissions can be beneficial or detrimental, depending on the context. But these studies vary greatly in terms of the breadth and detail of analysis for different effects pathways from congestion to emissions. A summary of the relevant literature on different congestion effects on emissions is shown in Table 1. These effects are discussed in more detail in the following sections.

\subsubsection{Direct Effects of Congestion}

The most salient direct impact of congestion is an increase in travel times (decrease in average travel speed), which increases average emissions rates per mile of travel when speeds are low (Barth \& Boriboonsomsin, 2008; Barth et al., 1999). This emissions rate increase is due both to increased engine loads from higher acceleration intensity and frequency during unsteady traffic and to longer operating time per unit distance at slower travel speeds (Barth \& Boriboonsomsin, 2008; Greenwood et al., 2007). However, studies have also shown that moderate travel speed reductions from excessively high speeds can reduce emissions rates per mile of travel (Barth \& Boriboonsomsin, 2008; Barth et al., 1999; Dijkema et al., 2008; Farzaneh, Schneider, \& Zietsman, 2010; S. Park et al., 2010). In other words, the direct effects of congestion on emissions rates vary across congestion levels. 
Table 1: Summary of Roadway Congestion Effects on Vehicle Emissions

\begin{tabular}{|c|c|}
\hline Congestion Effect & Impacts on Motor Vehicle Emissions \\
\hline $\begin{array}{l}\text { Decreased average } \\
\text { travel speeds }\end{array}$ & $\begin{array}{l}\text { - Increases emissions rates at very low speeds } \\
\text { (Barth \& Boriboonsomsin, 2008; Barth et al., 1999) } \\
\text { Decreases emissions rates for moderate speed reductions on } \\
\text { freeways } \\
\text { (Barth \& Boriboonsomsin, 2008; Barth et al., 1999; Dijkema et } \\
\text { al., 2008; Farzaneh, Schneider, \& Zietsman, 2010; S. Park et al., } \\
\text { 2010) }\end{array}$ \\
\hline $\begin{array}{l}\text { Increased speed } \\
\text { variability } \\
\text { (accelerations) }\end{array}$ & $\begin{array}{l}\text { - Increases emissions rates with acceleration intensity and } \\
\text { frequency; impact varies with travel speed and facility } \\
\text { (Barth \& Boriboonsomsin, 2008; Barth et al., 1999; Greenwood } \\
\text { et al., 2007) }\end{array}$ \\
\hline $\begin{array}{l}\text { Suppressed travel } \\
\text { demand } \\
\text { (or induced demand } \\
\text { with less congestion) }\end{array}$ & $\begin{array}{l}\text { - Less VMT decreases total emissions, but changes depend on the } \\
\text { road network and other factors; much research still needed } \\
\text { (Dowling, 2005; Noland \& Quddus, 2006; Stathopoulos \& } \\
\text { Noland, 2003) }\end{array}$ \\
\hline $\begin{array}{l}\text { Travel time } \\
\text { unreliability }\end{array}$ & $\begin{array}{l}\text { - No studies found on direct emissions effects (related to driving } \\
\text { behavior or traffic characteristics of non-recurring congestion) } \\
\text { - Indirectly, could suppress travel demand (Goodwin, 2004) and } \\
\text { so reduce VMT and emissions as above } \\
\text { - No studies found on other indirect effects on emissions (related } \\
\text { to routing, departure time, etc.) }\end{array}$ \\
\hline Trip rerouting & - Mixed effects possible (Nagurney, 2000); more research needed \\
\hline Departure time shifts & - No studies found \\
\hline Mode shift to transit & $\begin{array}{l}\text { Increases bus emissions, but smaller than savings from reduced } \\
\text { driving (Beevers \& Carslaw, 2005); more research needed }\end{array}$ \\
\hline Increased vehicle wear & $\begin{array}{l}\text { - No studies found on potential increased emissions rates with } \\
\text { increased vehicle wear }\end{array}$ \\
\hline $\begin{array}{l}\text { Increased vehicle } \\
\text { operating costs }\end{array}$ & $\begin{array}{l}\text { - No studies found on potential indirect emissions effects through } \\
\text { decreased travel demand }\end{array}$ \\
\hline $\begin{array}{l}\text { Freight operating cost } \\
\text { increases and potential } \\
\text { supply chain or freight } \\
\text { operations responses }\end{array}$ & $\begin{array}{l}\text { - Emissions impacts vary with route (Figliozzi, 2011) } \\
\text { - Congestion mitigation can increase freight VMT (Weisbrod et } \\
\text { al., 2001) and so increase emissions } \\
\text { - No studies found on potential emissions effects through } \\
\text { changing fleet mix, freight mode shift, or delivery time shifts }\end{array}$ \\
\hline
\end{tabular}




\subsubsection{Indirect Effects of Congestion}

Longer travel times due to consistent congestion suppress vehicle travel demand (Hymel et al., 2010). This the inverse of the induced demand caused by traffic flow (and travel time) improvements (DeCorla-Souza \& Cohen, 1999; Douglass Lee, Klein, \& Camus, 1999; Noland \& Cowart, 2000; Noland \& Lem, 2002). Using microscopic traffic simulation, research has shown that induced demand can increase total vehicle emissions at a bottleneck location after a traffic flow improvement (Noland \& Quddus, 2006; Stathopoulos \& Noland, 2003). If this is true, then suppressed travel demand has the potential to offset higher emissions rates per vehicle-mile and so reduce total emissions in congestion.

Travel behavior changes in response to congestion vary with the road network and other factors, and more detailed research is needed in this area. For example, vehicle travel demand can change by way of the frequency, distance, scheduling, travel mode, or routing of trips (Cervero, 2002; DeCorla-Souza \& Cohen, 1999). Different demand responses will have differing impacts on the total volume of emissions - in addition to the spatial-temporal allocation of emissions.

In addition to demand-suppressing long travel times, congestion causes travel time unreliability, another common indicator of poor performance for a roadway (ECMT, 2007; Schrank \& Lomax, 2007). The demand-suppressing effects of the disutility of unreliable travel times are not as well studied or quantified as for average travel times. Goodwin (2004) presumes they could exceed average travel speed effects on demand. 
Indeed, as an implication of support for this notion, Small, Winston, \& Yan (2005) estimate the value of reliability at about $50 \%$ to $100 \%$ of the value of travel time, while Brownstone \& Small (2005) estimate it at 95\%-140\%.

The emissions impacts of other facets of unreliability besides demand suppression (e.g. direct effects related to traffic characteristics during non-recurring congestion or other indirect effects related to routing, departure time, etc.) have not been quantified, to the best of the author's knowledge. More generally, the emissions differences between recurring and non-recurring congestion is in an area needing research - particularly because they are targeted with different types of mitigation strategies.

\subsection{Travel Demand Elasticity}

Given the importance of changing travel volumes for emissions effects, we here describe travel demand responses in more detail. Travel demand responses to changing congestion levels are typically assessed using travel demand elasticity to travel time, which is the percent change in travel volume (typically measured as vehicle-miles of travel (VMT)) with each percent change in travel time (see Litman (2011) for a discussion). Demand elasticity to travel time has a negative value because of the decreased attractiveness of longer-duration trips. Demand elasticity values vary with the roadway network, time range of interest, value of travel time in relation to other costs, trip length and purpose, and other local characteristics such as the amount of vacant land (DeCorla-Souza \& Cohen, 1999). 
The literature generally agrees on a range of -0.2 to -1.0 for likely vehicle travel demand elasticities to travel time (DeCorla-Souza \& Cohen, 1999; Goodwin, 1996; Jong \& Gunn, 2001; Douglass Lee et al., 1999; Noland, 2001; Noland \& Cowart, 2000). Larger absolute values for demand elasticity (more negative) are more applicable for longer time scales and situations with more travel options (in terms of modes, routes, destinations, etc.). Demand elasticity of VMT reflects net changes, and so ideally includes the aggregate affects of rerouting and changes in the number of trips. Perfectly inelastic (fixed) demand has an elasticity of 0 , and perfectly elastic demand (which implies a fixed travel time) would have an elasticity approaching negative infinity.

Demand elasticity to generalized costs is higher than demand elasticity to travel time alone, varying with the value of travel time in the context of total transportation costs. For example, Graham and Glaister (2004) point out that demand elasticity to travel time for personal travel is increasing because the value of time is an increasingly large portion of generalized driving costs (65\% by 2000$)$.

For freight the demand elasticity to generalized cost has been empirically estimated as a full order of magnitude greater than the freight demand elasticity to travel time alone (HLB Decision Economics Inc., 2008). This is logical since the other cost components for freight transport are proportionally higher than for personal travel. For freight vehicles, complex relationships exist between travel time and travel demand because time costs must be viewed in the context of supply chains, labor, and market 
costs. This complexity is discussed thoroughly by Weisbrod in NCHRP Report 463 (2001).

Demand elasticity to average travel speed can be simply estimated as the negative of demand elasticity to travel time. Table 2 presents a summary of travel demand elasticities to travel speed that have been reported in the literature (signs converted when originally reported as demand elasticity to travel time). This is by no means a comprehensive list, though it incorporates several meta-reviews of induced demand studies. For passenger-only and passenger-dominated general road travel (predominantly personal auto trips), elasticities of VMT demand to travel speed have been most commonly reported from 0.2 to 1.0 (DeCorla-Souza \& Cohen, 1999; Goodwin, 1996; Jong \& Gunn, 2001; Barr, 2000; Cohen, 1995; Williams \& Moore, 1990). Lower elasticities are more often found over shorter time scales, as many behavior modifications require time to realize.

\section{Table 2. Elasticity of Travel Demand (Distance) to Travel Speed from the}

\section{Literature}

\begin{tabular}{|l|l|l|}
\hline Source & Elasticity Values & Context \\
\hline (Jong \& Gunn, 2001) & $0.2-0.7$ & Passenger Travel \\
\hline (Barr, 2000) & $0.3-0.4$ & Passenger Travel \\
\hline (DeCorla-Souza \& Cohen, 1999) & $0.4-1.0$ & General \\
\hline (Cohen, 1995) & $0.6-1.8$ & General \\
\hline (Williams \& Moore, 1990) & $0.3-1.5$ & General \\
\hline (Goodwin, 1996) & $0.5-1.0$ & General \\
\hline (Oum, 1989) & $0.9-1.0$ & Freight \\
\hline (Abdelwahab, 1998) & 1.0 & Freight \\
\hline (HLB Decision Economics Inc., 2008) & $0.01-0.02$ & Freight \\
\hline
\end{tabular}

Signs have been reversed on values originally reported as elasticity to travel time 
Road/truck freight elasticities have been reported from 0.0 to 1.0 (HLB Decision Economics Inc., 2008; Oum, 1989; Abdelwahab, 1998). The freight elasticities, however, are based on much fewer studies, as fewer data are available and the situation is more complex. While demand elasticity is generally highly uncertain (as are most behavioral responses), this is particularly true for freight transport. Graham \& Glaister (2004) point out that freight travel demand in general is under-studied and not as well understood as passenger travel, and that while freight travel demand has traditionally been assumed to be inelastic, that is likely not the case.

The unique behavioral responses of freight vehicles is an important consideration for emissions because freight is moved by heavy-duty vehicles, which have higher emissions rates than light-duty vehicles - largely because of high gross vehicle weights (Brodrick et al., 2004). Heavy duty vehicles are also predominantly diesel-fueled, and diesel fuel has different emissions characteristics from gasoline (Scora, Boriboonsomsin, \& Barth, 2010), which powers most of the U.S. light-duty fleet (U.S. Environmental Protection Agency, 2009b).

The uncertainty of demand responses makes prediction of congestion and congestion mitigation effects on total emissions especially difficult to predict. A more indepth analysis of demand elasticity to speed or travel time is beyond the scope of this thesis, but there are many quality published papers that do exactly that. Meta-reviews of demand elasticity that might interest the reader include: Goodwin, Dargay, \& Hanly, 2004; Goodwin, 1992; Graham \& Glaister, 2004; and Oum, II, \& Yong, 1992. 


\subsection{Congestion Mitigation and Emissions}

Assuming isotropic conditions, congestion mitigation will have the opposite effects of congestion. Thus the engine operating inefficiencies of congested, low-speed vehicle travel have prompted suggestions for congestion mitigation targeting emissions reductions. Unfortunately, assessment of congestion mitigation strategies suffers the same limitations as estimates of congestion impacts and costs described above.

\subsubsection{Capacity-Based Strategies}

Capacity-based strategies (CBS) for reducing emissions ease congestion by increasing a roadway's vehicle throughput and so increase vehicle operating efficiency. CBS can increase capacity by increasing physical lane-miles or by increasing existing roadway efficiency and utilization through traffic flow improvements. The desired emissions benefit of congestion mitigation through CBS is reduced marginal emissions rates at higher traffic speeds. This approach presents the potential for induced demand.

A report by Dowling (2005) used travel demand modeling to estimate air quality effects of traffic flow improvements but yielded very large uncertainties (Noland \& Quddus, 2006). The conclusion of the report was that more research is needed "to better understand the conditions under which traffic-flow improvements contribute to an overall net increase or decrease in vehicle emissions." Other, more focused research on a limited spatial scale has shown that induced demand from traffic flow improvements make emissions rate reductions through CBS unlikely to reduce total emissions (Noland \& Quddus, 2006; Stathopoulos \& Noland, 2003). 


\subsubsection{Non-Capacity-Based Strategies}

As an alternative to CBS for emissions reductions, non-capacity based strategies (NCBS) aim to reduce emissions without increasing roadway capacity - by increasing vehicle efficiency at a given travel speed or by reducing the total amount of travel. As an example, Barth and Boriboonsomsin (2009) show that more efficient driving behavior on freeways can reduce greenhouse gas emissions by 10\%-20\% without a significant change in travel time, with more benefits at higher levels of congestion. NCBS also can directly target emissions through cleaner vehicles and fuels. Demand-side NCBS that reduce net travel volumes by methods such as road pricing can reduce emissions and also ease congestion (Beevers \& Carslaw, 2005).

Admittedly, the CBS/NCBS division does not cleanly categorize all possible approaches to emissions mitigation. For example, "road diets" or roadway capacity reductions are capacity-based strategies that increase (or at least maintain) congestion levels, but still with the potential for emissions reductions through suppressed travel demand. The most commonly suggested NCBS include some style of "eco-driving" (Barkenbus, 2010; Barth \& Boriboonsomsin, 2009), high-occupancy vehicle lanes (Boriboonsomsin \& Barth, 2007; Krimmer \& Venigalla, 2006), congestion pricing or road pricing (Beevers \& Carslaw, 2005; Johansson, 1997; Smyth \& Christodoulou, 2010), and speed-smoothing/steadying traffic management techniques such as variable speed limits and intelligent speed adaptation (Barth \& Boriboonsomsin, 2008; Mahmod et al., 2010; Wu et al., 2010). 


\subsection{Literature Summary}

While a great deal of work has been done in the field of motor vehicle emissions estimation, our understanding of the full congestion impacts on emissions is still limited. Generally, congestion decreases vehicle efficiency but also suppresses travel demand and the balance of these is not easily quantified. Too many estimates of congestion costs and impacts consider efficiency changes but simply neglect variable demand effects. Furthermore, those studies which do consider variable demand are typically highly context-specific, with unknown applicability to other situations. Thus, assumptions of congestion and emissions co-mitigation require more inspection. The objectives of this thesis are motivated by observation of these gaps in the state of knowledge, as described in the next chapter. 


\section{OBJECTIVES}

This research aims to alleviate some of the uncertainty about the relationship between congestion and emissions and the potential for congestion mitigation as an emissions reduction strategy. As illustrated in Figure 1, capacity-based congestion mitigation which increases travel speed can influence total emissions both through emissions rate reductions (due to increase vehicle operating efficiency) and increased travel volumes (due to travel behavior changes). The opposite can be said for increasing congestion: decreased travel speeds tend to increase emissions through increased emissions rates but simultaneously decrease emissions through lower travel volumes. Understanding the balance of these two effects pathways is the purview of this research.

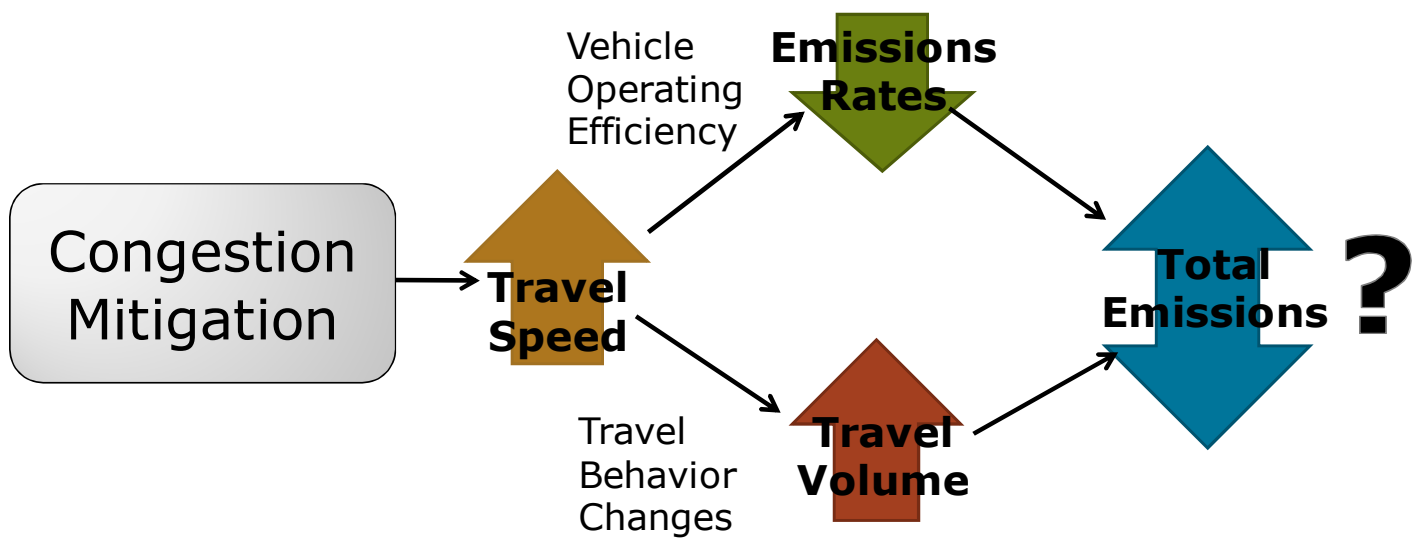

Figure 1. Congestion-Emissions Influence Paths

The specific objectives of this thesis are enumerated as follows:

1. Present a conceptual framework for assessing the impacts of congestion on emissions with minimal location specificity

2. Develop generalized relationships between travel speed and vehicle emissions - taking into consideration variable travel demand 
3. Describe situations in which capacity-based traffic congestion mitigation is likely to reduce motor vehicle emissions - with particular attention to the role of different vehicle classes

4. Compare capacity-based congestion mitigation with other emissions reduction strategies

5. Assess congestion performance measures and their applicability for emissions-related impacts

This research will address several gaps in the literature and shortcomings of the current body of knowledge by:

1. Using a variable travel demand approach (and not simple free-flow speed comparisons) to estimate congestion impacts on emissions

2. Developing generalized relationships between congestion and emissions that are comprehensible, expedient, and broadly applicable - and include emissions sensitivity to both travel speed and volume

3. Providing simple sketch-planning tools that can be applied anywhere with some simple assumptions and estimation of parameters

The effects pathways in Figure 1 are the motivation for the framework of the methodology, as described in the next chapter. 


\section{METHODOLOGY}

The macroscopic modeling in this study is designed to advance our understanding of the relationships between traffic congestion and vehicle emissions. We begin with a broad description of the conceptual framework of the modeling, and then continue with detailed descriptions of individual components. A summary list of the variables used in this analysis is provided in the prefatory pages of this thesis under "NOTATION", as a consolidated reference for the reader. The variables are all described in the following text of Chapter 4.

\subsection{Conceptual Framework}

There are many direct and indirect influence paths from congestion to emissions. A primary challenge of this research is to include as many effect pathways as possible, aiming for a comprehensive and yet still broadly applicable approach. Our approach to accomplishing this is suggested by the diagram in Figure 1. This diagram illustrates two aggregate effect pathways, each representing multiple effects. Congestion level is indicated by average travel speed. Then on the top pathway, myriad vehicle operating conditions (speeds, accelerations, idling) with varying congestion level are quantified in the changing average emissions rates (per vehicle-mile). On the bottom pathway, many diverse traveler behavioral responses are represented by net changes in travel demand volume.

If our primary interest is the changes in total emissions with changes in congestion level, then we do not need to model absolute vehicle volumes or emissions rates, but individual and join changes with changes in travel speed. We will revisit this 
framework at the end of the Methodology chapter, after introducing key variables and relationships. In the following sections we describe processes for traffic and emissions modeling and develop the equations that relate changes in total emissions to changes in speed.

\subsection{Macroscopic Traffic Modeling}

\subsubsection{Rate of Travel}

Travel demand modelers use demand volume-travel speed relationships to estimate the average speed over a road section (with respect to the traveler) based on demand flow, road capacity, and other parameters. This analysis employs the well-known Bureau of Public Roads (BPR) model for this purpose (Bureau of Public Roads, 1964). The BPR volume-travel time function calculates the average travel rate, $\bar{t}$, in time per unit distance, as a function of the effective demand volume, $q^{\prime}$, in passenger-cars per lane per unit time, as

$$
\bar{t}=t_{o}\left(1+\alpha\left(q^{\prime} / c\right)^{\beta}\right)
$$

where $t_{o}$ is the free-flow travel rate, $c$ is the roadway capacity in passengercars/lane/time, and $\alpha$ and $\beta$ are dimensionless parameters. The average travel speed, $\bar{v}$, in

distance per unit time, is then simply the inverse of average travel rate, $\bar{v}=1 / \bar{t}$. We use $\alpha=0.15$ and $\beta=7$ from Hansen et al. (2005), calibrated for the Portland region. This model and these parameter values are used illustratively, while recognizing that the selection of 
a volume-speed relationship can have a significant impact on total emissions calculations (Bai et al., 2007).

\subsubsection{Vehicle Classes and Effective Flows}

The effective vehicle flow rate for the BPR function, $q^{\prime}$, is the volume of vehicles traversing a section of roadway, converted to passenger-car equivalent (PCE) units. The PCE value for each passing vehicle is the amount of roadway capacity that the vehicle occupies, referenced to the capacity occupied by a typical passenger car. This is used to adjust for the larger spatial requirements for larger and heavier vehicles in traffic. Typical PCE values for trucks range from 1.5 for level terrain to 4.5 for mountainous terrain (Transportation Research Board, 2000). There are more complex ways to account for the differing performance of trucks in congestion (see Yun, White, Lamb, \& Y. Wu (2005) for an example), but the PCE method is considered sufficient for the macroscopic analysis performed here.

Let $f_{j}$ be the fractional fleet composition (by travel volume) of each vehicle class $j$ in the set of vehicle classes $J$. Further, let $P C E_{j}$ be the PCE value for all vehicles of class $j$ and $q_{j}$ be the volume flow rate (in number of vehicles per unit time) of vehicle of class $j$. If $q$ is the total volume flow rate of all vehicles (in number of vehicles per unit time - not adjusted for PCE), then $q_{j}=f_{j} \cdot q$. Furthermore, if the vehicle classes $j$ in $J$

are mutually exclusive and exhaustive, then $\sum_{j \in J} f_{j}=1$ and so $\sum_{j \in J} q_{j}=q$. Finally, the effective flow rate $q^{\prime}$ can be calculated from these variables as

$$
q^{\prime}=\sum_{\mathrm{j} \in \mathrm{J}}\left(P C E_{j} \cdot q_{j}\right)=q \cdot \sum_{\mathrm{j} \in \mathrm{J}}\left(P C E_{j} \cdot f_{j}\right)
$$




\subsubsection{Level of Service Indicators}

As a qualitative congestion reference in this thesis, we use the level-of-service (LOS) indicators for basic freeway sections described in the Highway Capacity Manual (HCM) (Transportation Research Board, 2000). In the HCM, freeway LOS A through F are based on traffic density thresholds where LOS F is the most congested. We employ average travel speeds for each freeway LOS following Barth et al. (1999), who calculated average travel speeds from EPA driving schedules.

\subsection{Emissions Rates}

\subsubsection{Emissions Rate Modeling}

Average vehicle emissions rates are estimated using the MOtor Vehicle Emissions Simulator (MOVES) 2010, the latest average-speed emissions model from the U.S. Environmental Protection Agency (EPA) (2009a). Emissions rates (in grams per vehicle-mile) are modeled using estimated on-road vehicles in the Portland, Oregon metropolitan region for the year 2010. More information on the modeled vehicles is in the next section. The modeled pollutants are $\mathrm{CO}_{2} \mathrm{e}$ (greenhouse gases in carbon dioxide equivalent units), $\mathrm{CO}$ (carbon monoxide), $\mathrm{NO}_{\mathrm{x}}$ (nitrogen oxides), $\mathrm{PM}_{2.5}$ (particulate matter smaller than 2.5 microns), and HC (hydrocarbons). Where available, countyspecific inputs are used (meteorology, vehicle inspection and maintenance program, fuel formulation), and national averages are used for other model inputs (vehicle age distributions). The MOVES model outputs emissions rates in 16 average-speed bins for 17 emissions Source Types (combinations of vehicle class and fuel type) for 4 different seasons and 24 hours of the day on urban freeway (restricted) and urban arterial 
(unrestricted) facilities. The average speed bins are in $5 \mathrm{mph}$ increments, up to $75 \mathrm{mph}$. The modeled emissions are running exhaust and evaporative emissions; refueling, brake/tire wear, and start emissions are not included. Particulate resuspension is not modeled by MOVES.

The average-speed emissions modeling approach estimates emissions for average travel speeds using facility-specific driving patterns (speed profiles). These driving patterns (also called "drive cycles" or "drive schedules") are composed of measured, archetypal combinations of acceleration, deceleration, cruise, and idle behavior at various average travel speeds on specific facilities, collected on-road in various U.S. cities (see MOVES documentation for details). Drive patterns effectively represent typical congested conditions for emissions modeling, as long as they are representative of realworld driving (Smit et al., 2008). They generally do not represent unique microscopic traffic characteristics and so cannot be used to model individual features in congestion (e.g. weaving sections), but they are appropriate for a macroscopic analysis such as performed here. For robustness, comparison analysis is also done using emissions rates published by Boulter et al. (2009) and Barth \& Boriboonsomsin (2008).

\subsubsection{Vehicle Fleet Composition}

For this analysis the MOVES emissions Source Types are combined into composite vehicle fleets based on the estimated distribution of 2010 Portland freeway vehicle miles traveled (VMT) by Source Type. First the percentage of freeway travel in 6 Highway Performance Monitoring System (HPMS) vehicle type classes is estimated using length-based classifications from 14 inductive dual-loop detector stations on 
Portland metropolitan freeways for 2009 (Oregon Department of Transportation, 2010). National-level 2010 freeway VMT and vehicle population estimates from the EPA (U.S. Environmental Protection Agency, 2010) are used for additional detail where lengthbased classifications cannot discriminate MOVES emissions Source Types (between passenger cars and passenger trucks for example).

The method to estimate fleet composition is summarized in following steps:

1) Estimate fraction of daily freeway VMT in each of 6 HPMS vehicle classes (10: motorcycles, 20: passenger cars, 30: other two-axle, four-tire single unit vehicles, 40: buses, 50: single-unit trucks, and 60: combination trucks)

a) Collect length-based vehicle classification data for 2009 from 14 inductive dualloop detector stations on Portland area freeways (Oregon Department of Transportation, 2010)

b) Average across stations for fraction of daily VMT in each of the 13 Federal Highway Administration (FHWA) vehicle classes

c) Combine the 13 FHWA vehicle class fractions into five HPMS vehicle classes (10: motorcycles, 20/30: all two-axle, four-tire single unit vehicles, 40: buses, 50: single-unit trucks, and 60: combination trucks); HPMS vehicle classes 20 and 30 are combined because length-based discrimination is unreliable - based both on observation of inconsistent data among stations and on HPMS documentation (Federal Highway Administration, 2001)

d) Use EPA estimates of 2010 freeway VMT fractions to separate HPMS vehicle classes 20 and 30 
i) EPA documentation provides national-level guidance for 1999 total VMT by HPMS vehicle class, with growth factors to estimate 2010 total VMT by HPMS vehicle class (U.S. Environmental Protection Agency, 2010)

ii) The same document provides estimates of the fraction of total VMT on urban restricted (freeway) facilities for each HPMS vehicle class (U.S.

Environmental Protection Agency, 2010)

iii) The resulting EPA estimates of 2010 freeway VMT fractions agree well with the combined 2009 Portland-area vehicle class distribution of freeway daily VMT

2) Separate each HPMS vehicle class into MOVES emissions Source Types

a) Use EPA estimates of 2010 vehicle populations by MOVES Source Type to apportion fractional VMT to MOVES Source Types within each HPMS vehicle class

b) The same EPA document used above also provides national-level guidance for 1999 vehicle populations by MOVES Source Type, with growth factors to estimate 2010 populations (U.S. Environmental Protection Agency, 2010)

c) An example of different MOVES Source Types within an HPMS vehicle class is the separation of Passenger Trucks from Light Commercial trucks in HPMS vehicle class 30

3) Estimate gasoline/diesel fuel splits for MOVES Source Types

a) Again, in the same document as used above the EPA provides national-level guidance for estimation of diesel fractions for each vehicle class (U.S. Environmental Protection Agency, 2010) 
b) Assumed diesel fractions from EPA documentation are $0.4 \%$ for Passenger Cars, 1\% for Passenger Trucks, 6\% for Light Commercial Trucks, $70 \%$ for Single Unit Trucks, and $100 \%$ for Combination (trailer) Trucks

c) Assume buses are $100 \%$ diesel

d) Assume all non-diesel vehicles use gasoline fuel

The resulting fleet composition from following these steps is shown in Table 3. Details on each of the MOVES emissions Source Types can be found in the MOVES documentation (U.S. Environmental Protection Agency, 2009a). After estimation of the full fleet composition, the emissions Source Types are also combined into light duty (LD) and heavy duty (HD) vehicle fleets. The LD vehicle fleet includes MOVES Source Type ID's below 40 (motorcycles, passenger cars, passenger trucks, and single-unit two-axle light commercial trucks under 19,500 lbs Gross Vehicle Weight Rating (GVWR)). The HD vehicle fleet includes MOVES Source Type ID's above 40 (buses, combination trucks, and other heavy trucks over 19,500 lbs GVWR). Using this partition, the full fleet is composed of $8.9 \% \mathrm{HD}$ vehicles.

As stated above, for each vehicle-fuel combination the MOVES model outputs emissions rate estimates in 16 average-speed bins for 4 different seasons and 24 hours of the day on freeway and arterial facilities. For each of the LD, HD, and Full vehicle fleets, composite emissions rates for each pollutant-speed-season-hour-facility combination are calculated using weighted averages. The weights are based on the percentages of fleet composition shown in the fourth column of Table 3. 
Table 3. MOVES Source Type Distribution

\begin{tabular}{|c|c|c|c|c|}
\hline MOVES Source Type & Vehicle Type & Fuel & $\%$ of Fleet & \\
\hline 11 & Motorcycle & Gas & 0.43 & \multirow{7}{*}{ 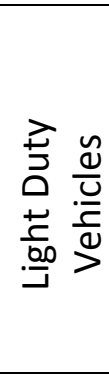 } \\
\hline 21 & Passenger Car & Gas & 52.83 & \\
\hline 21 & Passenger Car & Diesel & 0.21 & \\
\hline 31 & Passenger Truck & Gas & 27.92 & \\
\hline 31 & Passenger Truck & Diesel & 0.28 & \\
\hline 32 & Light Commercial Truck & Gas & 8.86 & \\
\hline 32 & Light Commercial Truck & Diesel & 0.57 & \\
\hline 41 & Intercity Bus & Diesel & 0.04 & \multirow{10}{*}{ 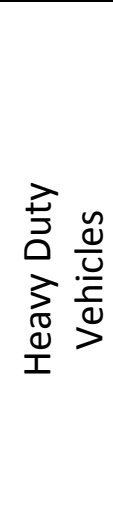 } \\
\hline 42 & Transit Bus & Diesel & 0.03 & \\
\hline 43 & School Bus & Diesel & 0.29 & \\
\hline 51 & Refuse Truck & Diesel & 0.07 & \\
\hline 52 & Single Unit Short Haul Truck & Gas & 1.00 & \\
\hline 52 & Single Unit Short Haul Truck & Diesel & 2.34 & \\
\hline 53 & Single Unit Long Haul Truck & Gas & 0.06 & \\
\hline 53 & Single Unit Long Haul Truck & Diesel & 0.14 & \\
\hline 61 & Combination Short Haul Truck & Diesel & 2.84 & \\
\hline 62 & Combination Long Haul Truck & Diesel & 2.11 & \\
\hline
\end{tabular}

Let $f_{s}$ be the fractional fleet composition (by VMT) of each Source Type $s$ in the set of Source Types $S$, and let $e_{s}$ be the spatial marginal emissions rates (in mass per vehicle-distance) for each Source Type $s$. Further, let $j$ be a composite vehicle class composed of a subset of Source Types in $S, S_{j} \subseteq S$ (such as all LD vehicles). Then the composite average emissions rate, $e_{j}$, for vehicle class $j$ can be calculated as

$$
e_{j}=\sum_{s \in S_{j}}\left(\frac{f_{f_{s}} \cdot e_{s}}{\sum_{s \in S_{j}} f_{s}}\right)=\frac{\sum_{s \in S_{j}}\left(f_{s} \cdot e_{s}\right)}{f_{j}}
$$

since $\sum_{s \in S_{j}} f_{s}=f_{j}$. Average emissions rates from the full vehicle fleet, $\bar{e}$, can be seen as a special case of $e_{j}$ where $S_{j} \equiv S$ and $\sum_{s \in S_{j}} f_{S}=1$. Alternatively, if $J$ is a set of mutually exclusive and exhaustive vehicle classes $j$, then $S \equiv \cup_{j \in J} S_{j}$ and 


$$
\bar{e}=\sum_{s \in S}\left(f_{s} \cdot e_{S}\right)=\sum_{j \in J}\left(\sum_{s \in S_{j}}\left(f_{s} \cdot e_{S}\right)\right)=\sum_{j \in J}\left(f_{j} \cdot e_{j}\right)
$$

\subsubsection{Marginal Emissions Rates as a Function of Speed}

To generate emissions rate versus average speed curves, emissions rates are fitted to a function of average speed for each pollutant-season-hour-facility combination. The discrete average-speed-bin emissions rates are least-squares fitted to an exponentiated polynomial of speed following previous emissions research (Barth \& Boriboonsomsin, 2008; Sugawara \& Niemeier, 2002). The functional form for vehicle class-average spatial marginal emissions rates, $e_{j}$, as a function of vehicle class-average speed, $v_{j}$, for each vehicle class $j$ is

$$
e_{j}\left(v_{j}\right)=\exp \left(\sum_{i=0}^{n} a_{i, j} \cdot v_{j}^{i}\right)
$$

where $a_{i, j}$ are fitted parameters, $e_{j}$ is in grams per vehicle-mile, and $v_{j}$ is in miles per hour (mph). We use $n=4$, again following previous research (Barth \& Boriboonsomsin, 2008; Sugawara \& Niemeier, 2002). We can similarly calculate the special case of fullfleet average emissions rates as

$$
\bar{e}(\bar{v})=\exp \left(\sum_{i=0}^{n} a_{i} \cdot \bar{v}^{i}\right)
$$

Full-fleet average temporal marginal emissions rates, $\bar{e}^{\prime}$, (in grams per vehiclehour of travel) are simply the product of $\bar{e}$ and $\bar{v}$. Thus from Equation $6, \bar{e}^{\prime}$ can be modeled as a function of average travel speed $\bar{v}$ using

$$
\bar{e}^{\prime}(\bar{v})=\bar{e} \cdot \bar{v}=\exp \left(\sum_{i=0}^{n} a_{i} \cdot \bar{v}^{\mathrm{i}+1}\right)
$$

In the same way, temporal marginal emissions rates for each vehicle class can be modeled using $a_{i, j}$ and $v_{j}$ 


\subsubsection{Marginal Emissions Rates as a Function of Volume}

Using a travel volume-speed relationship, marginal emissions rates per vehiclemile can also be estimated as a function of the travel demand volume, $q$, or the effective travel demand volume, $q^{\prime}$. A volume-speed model (such as the BPR function described above) relates $q^{\prime}$ to average speed $\bar{v}$ - which allows a transformation of $\bar{e}(\bar{v})$ to $\bar{e}\left(q^{\prime}\right)=$ $\bar{e}\left(\bar{v}\left(q^{\prime}\right)\right)$. Using the fitted parameters $a_{i}$ from Equation 6 and the BPR model shown in Equation 1 along with the relationship $\bar{v}=1 / \bar{t}$, we can calculate

$$
\bar{e}\left(q^{\prime}\right)=\exp \left(\sum_{i=0}^{n} a_{i} \cdot \bar{v}\left(q^{\prime}\right)^{i}\right)=\exp \left(\sum_{i=0}^{n} a_{i} t_{o}^{-i}\left(1+\alpha\left(q^{\prime} / c\right)^{\beta}\right)^{-i}\right)
$$

A similar approach was used by Sugawara \& Niemeier (2002) to estimate marginal emissions costs on a network link for an emissions-minimizing network assignment algorithm. If the vehicle class-average speed is the same as the average travel speed for all vehicles, $v_{j}=\bar{v}$, then we can similarly estimate $e_{j}$ from $q^{\prime}$ by substituting $a_{i, j}$ for $a_{i}$ in Equation 8.

We also propose a simpler formulation of $\bar{e}\left(q^{\prime}\right)$ which approximates the form of the BPR function (Equation 1), using four fitted parameters: $b_{0}, b_{1}, b_{2}, m$, and capacity $c$ :

$$
\bar{e}\left(q^{\prime}\right)=b_{0}+b_{1}\left(q^{\prime} / c\right)+b_{2}\left(q^{\prime} / c\right)^{m}
$$

The fitted parameters $b_{0}, b_{1}, b_{2}$, and $m$ are estimated by minimizing the square error of $\bar{e}\left(q^{\prime}\right)$ with respect to emissions rates from Equation 8, using $q^{\prime}$ as the independent 
variable. Both Equations 8 and 9 can easily be transformed to calculate $\bar{e}(q)$ instead of $\bar{e}\left(q^{\prime}\right)$ using estimates of $P C E_{j}$ and $f_{j}$ with Equation 2.

\subsection{Total Corridor Emissions}

Total emissions, $E_{j}$ (in mass per time, per length of roadway), from all on-road vehicles of class $j$ passing through a corridor are simply the product of the spatial marginal emissions rate, $e_{j}$, and the traffic volume flow rate $q_{j}$. Cumulative total corridor emissions, $E$, from on-road vehicles of all vehicle classes $j$ in the set of mutually exclusive and exhaustive vehicle classes $J$, per unit length of road per unit of time, are then

$$
E=\sum_{\mathrm{j} \in \mathrm{J}} E_{j}=\sum_{\mathrm{j} \in \mathrm{J}}\left(q_{j} \cdot e_{j}\right)=q \cdot \sum_{\mathrm{j} \in \mathrm{J}}\left(f_{j} \cdot e_{j}\right)=q \cdot \bar{e}
$$

\subsection{Travel Demand Elasticity}

The concept of travel demand elasticity is discussed, analyzed, and assessed thoroughly in the literature, as described in Section 2.5. We use demand elasticity here as the most established way of accounting for broad behavioral responses without introducing numerous other parameters to this analysis (such as network characteristics, trip characteristics, and other non-time cost components).

First we define the elasticity of travel demand volume $q_{j}$ (in number of vehicles

per unit time) to travel rate $t_{j}$ (in time per unit distance) for vehicles of class $j$ as $\eta_{q_{j}}^{t_{j}}$, which can be calculated

$$
\eta_{q_{j}}^{t_{j}}=\frac{t_{j}}{q_{j}} \cdot \frac{\partial q_{j}}{\partial t_{j}}
$$


This is the point elasticity at $\left(t_{j}, q_{j}\right)$. The linear or mid-point arc elasticity between two travel rate/demand volume conditions $\left(t_{j_{1}}, q_{j_{1}}\right)$ and $\left(t_{j_{2}}, q_{j_{2}}\right)$ is calculated as

$$
\eta_{q_{j}}^{t_{j}}=\frac{\left(t_{j_{2}}+t_{j_{1}}\right) / 2}{\left(q_{j_{2}}+q_{j_{1}}\right) / 2} \cdot \frac{\left(q_{j_{2}}-q_{j_{1}}\right)}{\left(t_{j_{2}}-t_{j_{1}}\right)}=\frac{\left(t_{j_{2}}+t_{j_{1}}\right)\left(q_{j_{2}}-q_{j_{1}}\right)}{\left(q_{j_{2}}+q_{j_{1}}\right)\left(t_{j_{2}}-t_{j_{1}}\right)}
$$

(see Litman, 2011). Using point elasticity, the elasticity of travel demand volume $q_{j}$ to changes in average travel speed $v_{j}$ for vehicles of class $j$, represented as $\eta_{q_{j}}^{v_{j}}$, is then

$$
\eta_{q_{j}}^{v_{j}}=\frac{v_{j}}{q_{j}} \cdot \frac{\partial q_{j}}{\partial v_{j}}=\frac{v_{j}}{q_{j}} \cdot \frac{\partial q_{j}}{\partial t_{j}} \cdot \frac{\partial t_{j}}{\partial v_{j}}=-\eta_{q_{j}}^{t_{j}}
$$

since $v_{j}=1 / t_{j}$ and $\frac{\partial t_{j}}{\partial v_{j}}=-v_{j}^{-2}$.

Travel demand elasticity in the literature generally addresses net changes in total travel distance (i.e. VMT) with changes in average travel rates or average travel speeds. The changes in VMT are the result of a combination of changing number of vehicle trips and changing vehicle trip distances, through various pathways (Cervero, 2002). The changes are net changes because travel speed changes can have offsetting effects. For example, with a travel speed reduction some vehicle trips could be eliminated because of the increased time costs (reducing VMT), while other vehicle trips could be elongated because of rerouting (increasing VMT).

If $L$ is the length of a corridor under study, then the total travel demand distance on the corridor for vehicle class $j$ (in vehicle-miles traveled per unit of analysis time) is

$$
V M T_{j}=q_{j} \cdot L
$$

The demand elasticity as assessed in the literature is best represented as $\eta_{V M T_{j}}^{v_{j}}$, the elasticity of $V M T_{j}$ to average travel speed on the corridor, $v_{j}$. If we assume that all 
changes in travel demand resulting from travel speed changes on the corridor are represented as changes in volume, $q_{j}$, on the fixed-length corridor, then

$$
\eta_{V M T_{j}}^{v_{j}}=\frac{v_{j}}{V M T_{j}} \cdot \frac{\partial V M T_{j}}{\partial v_{j}}=\frac{v_{j}}{q_{j} \cdot L} \cdot \frac{\partial\left(q_{j} \cdot L\right)}{\partial v_{j}}=\frac{v_{j}}{q_{j}} \frac{\partial q_{j}}{\partial v_{j}}=\eta_{q_{j}}^{v_{j}}
$$

This allows us to use estimates of $\eta_{V M T_{j}}^{v_{j}}$ from the literature to approximate $\eta_{q_{j}}^{v_{j}}$.

The effect of the assumption that $\eta_{q_{j}}^{v_{j}}=\eta_{V M T_{j}}^{v_{j}}$ is that all net travel demand (VMT) change related to the facility of interest is realized on the facility itself. The advantage of this approach is that it avoids specification of the characteristics of the trip outside the corridor, the broader roadway network, and the travel behavior responses. The disadvantage is that some of the net travel demand (VMT) change is actually occurring on other facilities. Using $\eta_{q_{j}}^{v_{j}}=\eta_{V M T_{j}}^{v_{j}}$ accurately represents the net change in the quantity of VMT, but it neglects the changing characteristics of VMT (the distribution by facility type, speed, etc.). But note that the utilization of demand elasticity itself involves a potential redistribution of VMT, since it only represents net changes in VMT. For estimating emissions effects, the assumption that $\eta_{q_{j}}^{v_{j}}=\eta_{V M T_{j}}^{v_{j}}$ will be most accurate when the emissions rates on the facility of interest are similar to those on other affected facilities.

The general assessment of $\eta_{V M T_{j}}^{v_{j}}$ from the literature is in the range of 0.2 to 1.0, as described in Section 2.5. This value, however, can vary with vehicle class, depending on the trip purposes (take the goods movement dominance of HD vehicle demand, for example). Beyond the average-speed effects on demand represented by $\eta_{V M T_{j}}^{v_{j}}$, traffic 
instability and unreliability also increase the costs of travel (Bigazzi \& Figliozzi, 2011), and thus can influence the travel demand volume. We assume no travel unreliability effects on travel demand for this analysis. Thus the results here will be conservative, as incorporating unreliability relationships would likely increase the demand elasticity to traffic speed/congestion level changes (Goodwin, 2004).

\subsection{Emissions Gradients and Elasticities}

The gradients of total and marginal emissions rates can be calculated using the derivates of $E_{j}$ and $e_{j}$.

\subsubsection{Emissions Changes with Travel Speed}

In this section we formulate equations which represent how emissions rates and total emissions, $e_{j}$ and $E_{j}$, vary with changes in $v_{j}$. This considers both $q_{j}$ and $e_{j}$ to be functions of $v_{j}$-i.e. variable demand volume and emissions rates with changing average speed. With marginal emissions rates, $e_{j}$, defined as in Equation 5, the emissions rate gradient for each vehicle class $j$ with respect to changes in $v_{j}$ is

$$
\frac{\partial e_{j}}{\partial v_{j}}=e_{j} \cdot \sum_{i=1}^{n}\left(i a_{i, j} v_{j}^{\mathrm{i}-1}\right)
$$

Expressed as the elasticity, $\varepsilon_{e_{j}}^{v_{j}}$, for vehicle class $j$ of emissions rates, $e_{j}$, to speed, $v_{j}$, this becomes

$$
\varepsilon_{e_{j}}^{v_{j}}=\frac{v_{j}}{e_{j}} \cdot \frac{\partial e_{j}}{\partial v_{j}}=\sum_{i=1}^{n}\left(i a_{i, j} v_{j}^{\mathrm{i}}\right)
$$

Using Equations 10, 13 and 17, we can then calculate the gradient in total emissions, $E_{j}$, from each vehicle class due to a change in class-average speed, $v_{j}$, as 


$$
\frac{\partial E_{j}}{\partial v_{j}}=\frac{\partial q_{j}}{\partial v_{j}} \cdot e_{j}+q_{j} \cdot \frac{\partial e_{j}}{\partial v_{j}}=\frac{E_{j}}{v_{j}}\left(\eta_{q_{j}}^{v_{j}}+\varepsilon_{e_{j}}^{v_{j}}\right)
$$

Expressed as a total emissions elasticity to speed for each vehicle class we have simply

$$
\varepsilon_{E_{j}}^{v_{j}}=\frac{v_{j}}{E_{j}} \cdot \frac{\partial E_{j}}{\partial v_{j}}=\eta_{q_{j}}^{v_{j}}+\varepsilon_{e_{j}}^{v_{j}}
$$

Combining Equations 17 and 19,

$$
\varepsilon_{E_{j}}^{v_{j}}=\eta_{q_{j}}^{v_{j}}+\sum_{i=1}^{n}\left(i a_{i, j} v_{j}^{\mathrm{i}}\right)
$$

and we see that the elasticity of total emissions to traffic speed changes for each vehicle class is dependent only on travel demand volume elasticity $\eta_{q_{j}}^{v_{j}}$, traffic speed $v_{j}$, and fitted emissions rate parameters $a_{i, j}$.

Let us define the average travel speed for all vehicles, $\bar{v}$, as the volume-weighted average of each vehicle class's average travel speed, $v_{j}$,

$$
\bar{v}=\sum_{\mathbf{j} \in \mathrm{J}}\left(f_{j} \cdot v_{j}\right)
$$

For the gradient of total emissions to average speed changes for all vehicles we have

$$
\frac{\partial E}{\partial \bar{v}}=\sum_{\mathrm{j} \in \mathrm{J}}\left[\frac{\partial E_{j}}{\partial \bar{v}}\right]=\sum_{\mathrm{j} \in \mathrm{J}}\left[\frac{\partial E_{j}}{\partial v_{j}} \cdot \frac{\partial v_{j}}{\partial \bar{v}}\right]=\sum_{\mathrm{j} \in \mathrm{J}}\left[\frac{E_{j}}{v_{j}} \cdot \varepsilon_{E_{j}}^{v_{j}} \cdot \frac{\partial v_{j}}{\partial \bar{v}}\right]
$$

Expressed as the elasticity of total emissions to average speed for all vehicles,

$$
\varepsilon_{E}^{\bar{v}}=\frac{\bar{v}}{E} \cdot \frac{\partial E}{\partial \bar{v}}=\frac{\bar{v}}{\bar{e}} \cdot \sum_{\mathrm{j} \in \mathrm{J}}\left[\frac{f_{j} e_{j}}{v_{j}} \cdot \varepsilon_{E_{j}}^{v_{j}} \cdot \frac{\partial v_{j}}{\partial \bar{v}}\right]
$$

If we assume that the absolute speed change is the same for all vehicle classes, $\frac{\partial v_{j}}{\partial \bar{v}}=1 \forall \mathrm{j} \in \mathrm{J}$, then for total emissions gradient and elasticity to average speed for all vehicles we have 


$$
\frac{\partial E}{\partial \bar{v}}=\sum_{\mathrm{j} \in \mathrm{J}}\left[\frac{\partial E_{j}}{\partial v_{j}}\right]=\sum_{\mathrm{j} \in \mathrm{J}}\left[\frac{E_{j}}{v_{j}} \cdot \varepsilon_{E_{j}}^{v_{j}}\right]
$$

and

$$
\varepsilon_{E}^{\bar{v}}=\frac{\bar{v}}{\bar{e}} \cdot \sum_{\mathrm{j} \in \mathrm{J}}\left[\frac{f_{j} e_{j}}{v_{j}} \cdot \varepsilon_{E_{j}}^{v_{j}}\right]
$$

Alternatively, if we assume that the proportional (percentage) speed change is the same for all vehicle classes, $\frac{\partial v_{j}}{\partial \bar{v}}=\frac{v_{j}}{\bar{v}} \forall \mathrm{j} \in \mathrm{J}$, then we have

$$
\frac{\partial E}{\partial \bar{v}}=\frac{q}{\bar{v}} \cdot \sum_{\mathrm{j} \in \mathrm{J}}\left[f_{j} \cdot e_{j} \cdot \varepsilon_{E_{j}}^{v_{j}}\right]
$$

and

$$
\varepsilon_{E}^{\bar{v}}=\frac{1}{\bar{e}} \cdot \sum_{\mathbf{j} \in \mathrm{J}}\left[f_{j} \cdot e_{j} \cdot \varepsilon_{E_{j}}^{v_{j}}\right]
$$

Therefore, in addition to the demand elasticity, traffic speed, and emissions fit parameters for each class needed to calculate, $\varepsilon_{E_{j}}^{v_{j}}$ (see Equation 20), elasticity of total emissions simply requires estimates of the fraction fleet composition, $f_{j}$.

Finally, for the average emissions rate from all vehicles, $\bar{e}$, if we assume that the fleet mix is unaffected by average speed changes, $\frac{\partial f_{j}}{\partial \bar{v}}=0 \forall \mathrm{j} \in \mathrm{J}$, and assume the same average-speed-change proportionality as above, $\frac{\partial v_{j}}{\partial \bar{v}}=\frac{v_{j}}{\bar{v}} \forall \mathrm{j} \in \mathrm{J}$, then

$$
\frac{\partial \bar{e}}{\partial \bar{v}}=\sum_{\mathbf{j} \in \mathrm{J}}\left(\frac{\partial f_{j}}{\partial \bar{v}} e_{j}+f_{j} \frac{\partial e_{j}}{\partial \bar{v}}\right)=\sum_{\mathbf{j} \in \mathrm{J}}\left(\frac{\partial e_{j}}{\partial v_{j}} \cdot \frac{\partial v_{j}}{\partial \bar{v}} \cdot f_{j}\right)=\frac{1}{\bar{v}} \cdot \sum_{\mathbf{j} \in \mathrm{J}}\left(e_{j} \cdot f_{j} \cdot \varepsilon_{e_{j}}^{v_{j}}\right)
$$

For the elasticity of average emissions rate, $\bar{e}$, to average speed, $\bar{v}$, for all vehicles (using the same assumptions),

$$
\varepsilon_{\bar{e}}^{\bar{v}}=\frac{\bar{v}}{\bar{e}} \cdot \frac{\partial \bar{e}}{\partial \bar{v}}=\frac{1}{\bar{e}} \cdot \sum_{\mathrm{j} \in \mathrm{J}}\left(e_{j} \cdot f_{j} \cdot \varepsilon_{e_{j}}^{v_{j}}\right)
$$




\subsubsection{Emissions Changes with Travel Volume}

In this section we formulate equations which represent how emissions rates and total emissions, $e_{j}$ and $E_{j}$, vary with changes in $q_{j}$. Here we consider $e_{j}$ to be a function of $q_{j}$ through $v_{j}\left(q_{j}\right)$ - by using a volume-speed relationship such as the BPR function. We first define the elasticity of travel speed, $v_{j}$, to changes in volume, $q_{j}$, for vehicle class $j$ as

$$
\varepsilon_{v_{j}}^{q_{j}}=\frac{q_{j}}{v_{j}} \frac{\partial v_{j}}{\partial q_{j}}
$$

and for all on-road vehicles as

$$
\varepsilon_{\bar{v}}^{q}=\frac{q}{\bar{v}} \frac{\partial \bar{v}}{\partial q}
$$

We can then calculate the elasticity of emissions rates, $e_{j}$, to travel volume, $q_{j}$, for each vehicle class as

$$
\varepsilon_{e_{j}}^{q_{j}}=\frac{q_{j}}{e_{j}} \cdot \frac{\partial e_{j}}{\partial q_{j}}=\frac{q_{j}}{e_{j}} \cdot \frac{\partial e_{j}}{\partial v_{j}} \cdot \frac{\partial v_{j}}{\partial q_{j}}=\varepsilon_{e_{j}}^{v_{j}} \cdot \varepsilon_{v_{j}}^{q_{j}},
$$

and for all on-road vehicles as

$$
\varepsilon_{\bar{e}}^{q}=\frac{q}{\bar{e}} \cdot \frac{\partial \bar{e}}{\partial q}=\frac{q}{\bar{e}} \cdot \frac{\partial \bar{e}}{\partial \bar{v}} \cdot \frac{\partial \bar{v}}{\partial q}=\varepsilon_{\bar{e}}^{\bar{v}} \cdot \varepsilon_{\bar{v}}^{q}
$$

The elasticity of total emissions $E_{j}$ to changes in travel volume $q_{j}$ for each vehicle class is calculated

$$
\varepsilon_{E_{j}}^{q_{j}}=\frac{q_{j}}{E_{j}} \cdot \frac{\partial E_{j}}{\partial q_{j}}=\frac{q_{j}}{E_{j}}\left(e_{j}+q_{j} \frac{\partial e_{j}}{\partial q_{j}}\right)=1+\varepsilon_{e_{j}}^{q_{j}}=1+\varepsilon_{e_{j}}^{v_{j}} \cdot \varepsilon_{v_{j}}^{q_{j}},
$$

and for all on-road vehicles

$$
\varepsilon_{E}^{q}=\frac{q}{E} \cdot \frac{\partial E}{\partial q}=1+\varepsilon_{\bar{e}}^{q}=1+\varepsilon_{\bar{e}}^{\bar{v}} \cdot \varepsilon_{\bar{v}}^{q}
$$


Since $\varepsilon_{e_{j}}^{v_{j}}$ and $\varepsilon_{\bar{e}}^{\bar{v}}$ can be calculated as described in the previous section, the remaining task is calculation of $\varepsilon_{v_{j}}^{q_{j}}$ and $\varepsilon_{\bar{v}}^{q}$. If we apply the BPR function, then for the general case inclusive of all on road vehicles, by differentiating Equation 1 we get

$$
\frac{\partial \bar{t}}{\partial q}=\frac{t_{o} \alpha \beta}{c} \cdot \frac{\partial q^{\prime}}{\partial q} \cdot\left(\frac{q^{\prime}}{c}\right)^{\beta-1}
$$

which allows us to compute

$$
\varepsilon_{\bar{v}}^{q}=\frac{q}{\bar{v}} \cdot \frac{\partial \bar{v}}{\partial \bar{t}} \cdot \frac{\partial \bar{t}}{\partial q}=-\frac{q \bar{v} t_{o} \alpha \beta}{c} \cdot \frac{\partial q^{\prime}}{\partial q} \cdot\left(\frac{q^{\prime}}{c}\right)^{\beta-1}=\frac{-\alpha \beta \frac{\partial q^{\prime}}{\partial q}}{\left(\left(\frac{c}{q^{\prime}}\right)^{\beta}+\alpha\right) \Sigma_{\mathrm{j} \in \mathrm{J}}\left(P C E_{j} \cdot f_{j}\right)} .
$$

It should be noted that these equations assume a certain, fixed relationship between $q^{\prime}$ (or q) and $\bar{v}$ (or $\bar{t}$ ) - namely, the BPR function. Importantly, the roadway capacity $c$ is a fixed parameter. For this reason using the volume-speed equations will only help us estimate the impacts of varying demand volumes on emissions when all other operational and roadway capacity factors are unchanged.

\subsection{Demand Elasticity for Break-Even Emissions Conditions}

Given the high uncertainty of true demand elasticity to travel speed, $\eta_{q_{j}}^{v_{j}}$, it can be informative to simply calculate a demand elasticity which represents break-even conditions from an emissions perspective, here denoted $\gamma_{q_{j}}^{v_{j}}$. Then we can compare $\gamma_{q_{j}}^{v_{j}}$ to a likely range of $\eta_{q_{j}}^{v_{j}}$ in order to predict the net emissions effects of a change in traffic speed, $v_{j}$. This is similar to the approach used by Noland $\&$ Quddus (2006) to predict the total emissions effects of a traffic flow change using microsimulation. 


\subsubsection{Break-Even Demand Elasticity by Vehicle Class}

From Equation 18 total emissions will remain constant with travel speed changes

for each vehicle class, $\frac{\partial E_{j}}{\partial v_{j}}=0$, when $\eta_{q_{j}}^{v_{j}}=-\varepsilon_{e_{j}}^{v_{j}}$. That is in addition to the trivial cases in which the traffic flow volume or the emissions rate from that vehicle class is

zero, $q_{j}=0$ or $e_{j}=0$. Therefore, from Equation 17 break-even demand elasticity, $\gamma_{q_{j}}^{v_{j}}$,

for vehicle class $j$ is calculated

$$
\gamma_{q_{j}}^{v_{j}}=-\varepsilon_{e_{j}}^{v_{j}}=-\sum_{i=1}^{n}\left(i a_{i, j} v_{j}^{\mathrm{i}}\right)
$$

For formulations of the emissions versus speed relationship, $e_{j}\left(v_{j}\right)$, other than Equation $5, \gamma_{q_{j}}^{v_{j}}$ can more generally be calculated from $\gamma_{q_{j}}^{v_{j}}=-\frac{v_{j}}{e_{j}} \cdot \frac{\partial e_{j}}{\partial v_{j}}$.

From Equations 19 and 38 follows that

$$
\varepsilon_{E_{j}}^{v_{j}}=\eta_{q_{j}}^{v_{j}}-\gamma_{q_{j}}^{v_{j}} .
$$

Thus, the elasticity of class-total emissions to traffic speed changes is the difference between the vehicle class's true demand elasticity to travel speed, $\eta_{q_{j}}^{v_{j}}$, and its break-even demand elasticity to travel speed, $\gamma_{q_{j}}^{v_{j}}$. When true demand elasticity exceeds break-even demand elasticity $\left(\eta_{q_{j}}^{v_{j}}>\gamma_{q_{j}}^{v_{j}}\right), \varepsilon_{E_{j}}^{v_{j}}$ is positive and traffic speed increases will increase total emissions from vehicle class $j$ (due to the dominance of induced demand). When true demand elasticity is less than break-even demand elasticity $\left(\eta_{q_{j}}^{v_{j}}<\gamma_{q_{j}}^{v_{j}}\right), \varepsilon_{E_{j}}^{v_{j}}$ is negative and total emissions from vehicle class $j$ will decrease from traffic speed increases (due to the dominance of increased efficiency). 
Since $\gamma_{q_{j}}^{v_{j}}$ in Equation 38 is only a function of $v_{j}$, not $q_{j}$, neither $\gamma_{q_{j}}^{v_{j}}$ nor $\varepsilon_{E_{j}}^{v_{j}}$ rely on a specific volume-speed relationship such as the BPR function. They can simply be calculated from the existing average speed $v_{j}$, the fitted emissions rate parameters $a_{i, j}$, and the true demand elasticity to speed $\eta_{q_{j}}^{v_{j}}$. The same independence of $\gamma_{q_{j}}^{v_{j}}$ and $\varepsilon_{E_{j}}^{v_{j}}$ from $q_{j}$ applies to other formulations of $e_{j}\left(v_{j}\right)$, as long as it is a function only of $v_{j}$.

\subsubsection{Graphical Method of Determining Break-Even Demand Elasticity}

As a graphical alternative, we can use the slope of the total emissions contour lines ("iso-emissions" lines) on the $v_{j}$ versus $q_{j}$ plane to estimate break-even emissions elasticity for a vehicle class $j, \gamma_{q_{j}}^{v_{j}}$. The total emissions contour slope can be calculated as the orthogonal vector to the total emissions gradient at any point. For total emissions $E_{j}\left(q_{j}, v_{j}\right)$ as a function of vehicle flow $q_{j}$ and traffic speed $v_{j}$, the total emissions gradient is $\nabla E_{j}\left(q_{j}, v_{j}\right)=\left(e_{j}, q_{j} \frac{\partial e_{j}}{\partial v_{j}}\right)$. Note that this deviates from the gradients of $E_{j}\left(q_{j}\right)$ and $E_{j}\left(v_{j}\right)$ shown in the previous sections since it disregards the relationship between $q_{j}$ and $v_{j}$.

The slope of the total emissions contour lines on the $q_{j}-v_{j}$ plane is the orthogonal vector to the gradient vector, $\left(-q_{j} \frac{\partial e_{j}}{\partial v_{j}}, e_{j}\right)$. Thus the iso-emissions lines follow the slope $\frac{\partial q_{j}}{\partial v_{j}}=\frac{-q_{j}}{e_{j}} \frac{\partial e_{j}}{\partial v_{j}}$, which is the relationship between $q_{j}$ and $v_{j}$ which represents emissions break-even conditions. Expressed as an elasticity, the iso-emissions slope is the breakeven demand elasticity $\gamma_{q_{j}}^{v_{j}}=\frac{v_{j}}{q_{j}} \frac{\partial q_{j}}{\partial v_{j}}=\frac{-v_{j}}{e_{j}} \frac{\partial e_{j}}{\partial v_{j}}=-\varepsilon_{e_{j}}^{v_{j}}$, which is the same as Equation 38. 


\subsubsection{Break-Even Demand Elasticity for a Mixed Fleet}

For mixed fleets of multiple vehicle classes, total emissions are unaffected by travel speed changes when $\frac{\partial E_{j}}{\partial v_{j}}=0$ for all vehicle classes, i.e. $\eta_{q_{j}}^{v_{j}}=\gamma_{q_{j}}^{v_{j}} \forall j \in J$. But not every class's total emissions need to be insensitive to speed changes in order to have a net zero change in total emissions with average travel speed changes. Some vehicle classes can increase class-total emissions while others decrease class-total emissions, off-setting each other.

We can see the potential for tradeoffs using Equation 27. If the travel speed change is proportionally equivalent for all vehicle classes, $\frac{\partial v_{j}}{\partial \bar{v}}=\frac{v_{j}}{\bar{v}} \forall j \in J$, then an emissions break-even condition exists when

$$
\varepsilon_{E}^{\bar{v}}=0=\sum_{j \in J}\left[f_{j} \cdot e_{j} \cdot \varepsilon_{E_{j}}^{v_{j}}\right] .
$$

For this to be true in cases other than $\varepsilon_{E_{j}}^{v_{j}}=0 \forall j \in J$, some vehicle classes must have positive $\varepsilon_{E_{j}}^{v_{j}}$ and others negative (since $f_{j}$ and $e_{j}$ will always be positive). More generally, for conditions when the speed changes vary by vehicle class as $\Delta v_{j}$, total emissions are unaffected by speed changes when

$$
\varepsilon_{E}^{\bar{v}}=0=\sum_{\mathrm{j} \in \mathrm{J}}\left[\frac{f_{j} e_{j}}{v_{j}} \cdot \varepsilon_{E_{j}}^{v_{j}} \cdot \Delta v_{j}\right]
$$

Here we see that trade-offs can come from positive and negative values of $\varepsilon_{E_{j}}^{v_{j}}$ and/or from speed changes in different directions (positive and negative values of $\Delta v_{j}$ ). 


\subsection{Other Data Used in the Analysis}

In addition to the data required for emissions and traffic modeling described above, in this research we make use of real-world traffic data from the PORTAL data archive at Portland State University (http:Ilportal2.its.pdx.edu). This traffic data archive extends back to 2004 with 20-second aggregated vehicle count, average speed, and detector occupancy data from hundreds of inductive dual-loop detectors on the Portland, Oregon metropolitan freeway system.

\subsection{Summary of Methodology}

As stated previously, the conceptual diagram in Figure 1 shaped the methodology of this research. Now that we have developed the necessary equations, we can revisit the conceptual framework and flush it out with notation and the core equation. Figure 2 shows the key pieces of the modeling framework expressed as elasticities (illustrated assuming a uniform vehicle fleet). The last equation in the figure, $\varepsilon_{E}^{\bar{v}}=\eta_{q}^{\bar{v}}+\varepsilon_{\bar{e}}^{\bar{v}}$, is the central equation of this research; it expresses the total emissions elasticity to speed as the joint effects of both pathways (vehicle efficiency through $\varepsilon_{\bar{e}}^{\bar{v}}$ and behavioral responses through $\left.\eta_{q}^{\bar{v}}\right)$. 


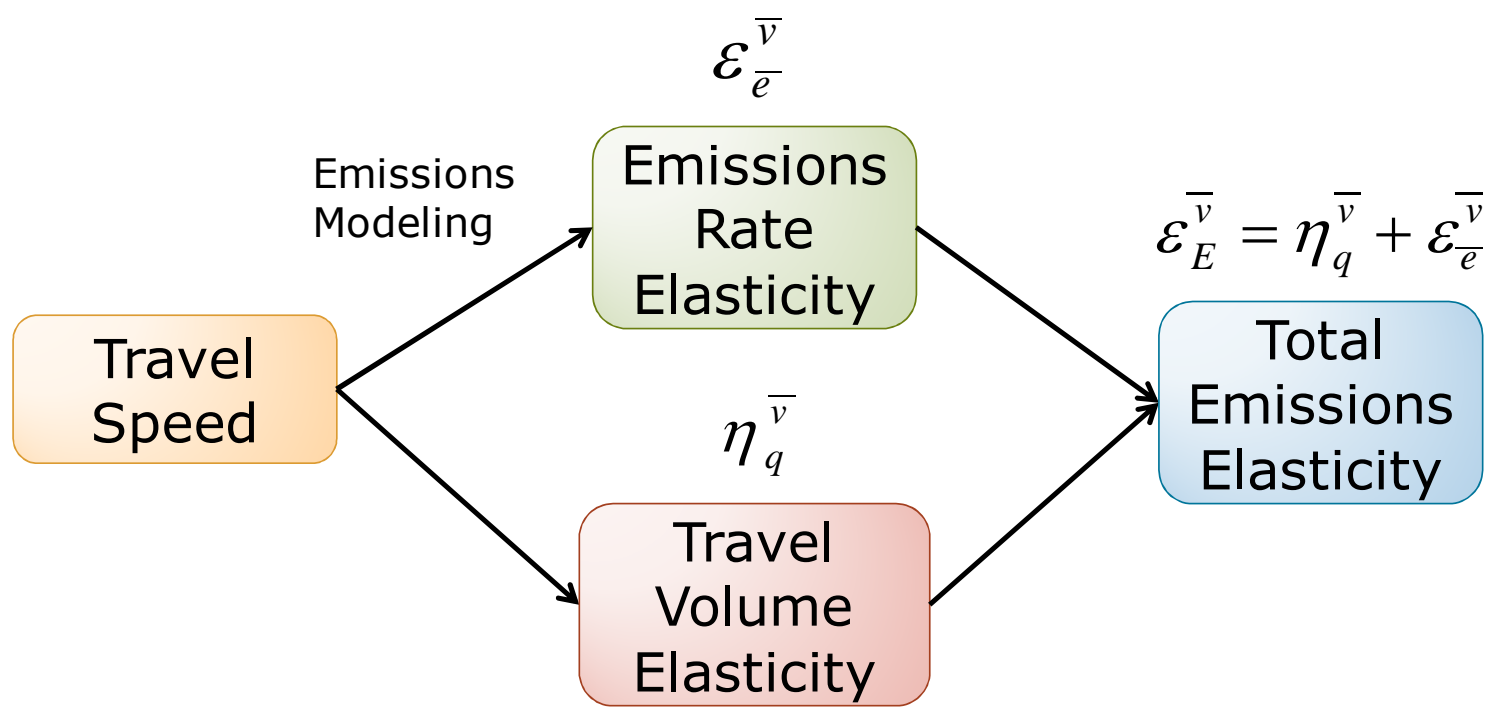

Figure 2. Conceptual Modeling Framework as Elasticities

Emissions rate elasticity, $\varepsilon_{\bar{e}}^{\bar{v}}$, can be analytically determined from emissions rate modeling (Equation 17). There is uncertainty associated with the emissions modeling, but emissions rates can be estimated for aggregate average driving conditions. Demand elasticity, $\eta_{q}^{\bar{v}}$, has more uncertainty because it depends on forecasting driver behavior changes. This driver behavior uncertainty motivates the estimation of break-even demand elasticity, $\gamma_{q}^{\bar{v}}$, which can also be calculated from the emissions rate modeling results (see Equation 38). Then a likely range of $\eta_{q}^{\bar{v}}$ can be compared with $\gamma_{q}^{\bar{v}}$ to assess the expected emissions effects of a travel speed change. This relatively simple approach allows estimation of the total emissions impacts of congestion or congestion mitigation, requiring only the emissions-speed curve fit parameters and an expected range for demand elasticity. In the next chapter we will see the results of this estimate using different emissions models. 


\section{FULL FLEET TOTAL EMISSIONS}

We first present the emissions results as they relate to the full vehicle fleet. In this chapter we treat the entire vehicle fleet as a unified class, where $S_{j} \equiv S$. The following sections show emissions rates per vehicle-mile as a function of speed on freeways and arterials, emissions rates per vehicle-hour, emissions rates as a function of volume, total emissions, and emissions elasticities. These results are for undefined roadways of each facility type.

\subsection{Spatial Emissions Rates (per Vehicle-Mile)}

The composite full-fleet average emissions rates, $\bar{e}$, are calculated as a special case of Equation 3 where $S_{j} \equiv S$ and $\sum_{s \in S_{j}} f_{s}=1$. Then the parameters $a_{i}$ are fitted from Equation 6 for $\bar{e}(\bar{v})$ by minimizing square error, using $n=4$. The data points for the curve fit are the modeled emissions rates at each of 16 average speed bins (in $5 \mathrm{mph}$ increments). The fitted parameters $a_{i}$ for the MOVES-based Emissions-Speed Curves (ESC) are shown in Table 4 for the full on-road fleet, for PM peak periods on freeways in April, 2010. These use $\bar{v}$ in mph and $\bar{e}$ in grams per vehicle-mile.

Table 4. Full-Fleet MOVES Emissions-Speed Curve Fit Parameters for Freeways

\begin{tabular}{|c|r|r|r|r|r|}
\hline Freeways & \multicolumn{1}{|c|}{$\mathbf{C O}_{\mathbf{2}} \mathbf{e}$} & \multicolumn{1}{c|}{$\mathbf{C O}$} & \multicolumn{1}{c|}{$\mathbf{P M}_{2.5}$} & \multicolumn{1}{c|}{$\mathbf{N O}_{\mathbf{x}}$} & \multicolumn{1}{c|}{$\mathbf{H C}$} \\
\hline$a_{0}$ & 8.191 & 2.885 & -1.223 & 1.897 & 0.3352 \\
\hline$a_{1}$ & -0.1826 & -0.1788 & -0.1769 & -0.1656 & -0.2040 \\
\hline$a_{2}$ & 0.006339 & 0.006629 & 0.006640 & 0.005830 & 0.006643 \\
\hline$a_{3}$ & $-9.690 \mathrm{E}-05$ & $-1.092 \mathrm{E}-04$ & $-1.127 \mathrm{E}-04$ & $-8.928 \mathrm{E}-05$ & $-1.012 \mathrm{E}-04$ \\
\hline$a_{4}$ & $5.357 \mathrm{E}-07$ & $6.518 \mathrm{E}-07$ & $6.724 \mathrm{E}-07$ & $4.936 \mathrm{E}-07$ & $5.674 \mathrm{E}-07$ \\
\hline
\end{tabular}


Plots of full-fleet freeway marginal emissions, $\bar{e}$, versus average travel speed, $\bar{v}$, are shown in Figure 3 for $\mathrm{CO}_{2} \mathrm{e}, \mathrm{CO}, \mathrm{PM}_{2.5}, \mathrm{NO}_{\mathrm{x}}$, and $\mathrm{HC}$. In addition to the ESC generated by MOVES for a 2010 Portland on-road fleet, comparison curves are plotted based on research by Boulter et al. (2009) and Barth \& Boriboonsomsin (2008). The Boulter curves are for European vehicles on unspecified facilities, with an approximately equivalent mix of vehicle types as the Portland 2010 modeled fleet, shown in Table 3. The Boulter curves are only drawn over their valid speed range. The Barth curve is for $\mathrm{CO}_{2}$ emissions (plotted with $\mathrm{CO}_{2} \mathrm{e}$ from MOVES), for a $\mathrm{LD}$ vehicle fleet from Southern California in 2005. As a qualitative reference, average speeds for different freeway levelof-service (LOS) indicators are included, as described in Section 4.2.3. Note that all three models estimate slightly different $\mathrm{CO}_{2}$ emissions: MOVES estimates $\mathrm{CO}_{2} \mathrm{e}$ (all greenhouse gases in $\mathrm{CO}_{2}$-equivalent units), Barth estimates direct emissions of $\mathrm{CO}_{2}$, and Boulter estimates ultimate $\mathrm{CO}_{2}$ (using the assumption that all the carbon content of the fuel eventually ends up as atmospheric $\mathrm{CO}_{2}$ ). Since $\mathrm{CO}_{2}$ dominates greenhouse gas emissions (U.S. Environmental Protection Agency, 2009), these emissions types should be comparable. 

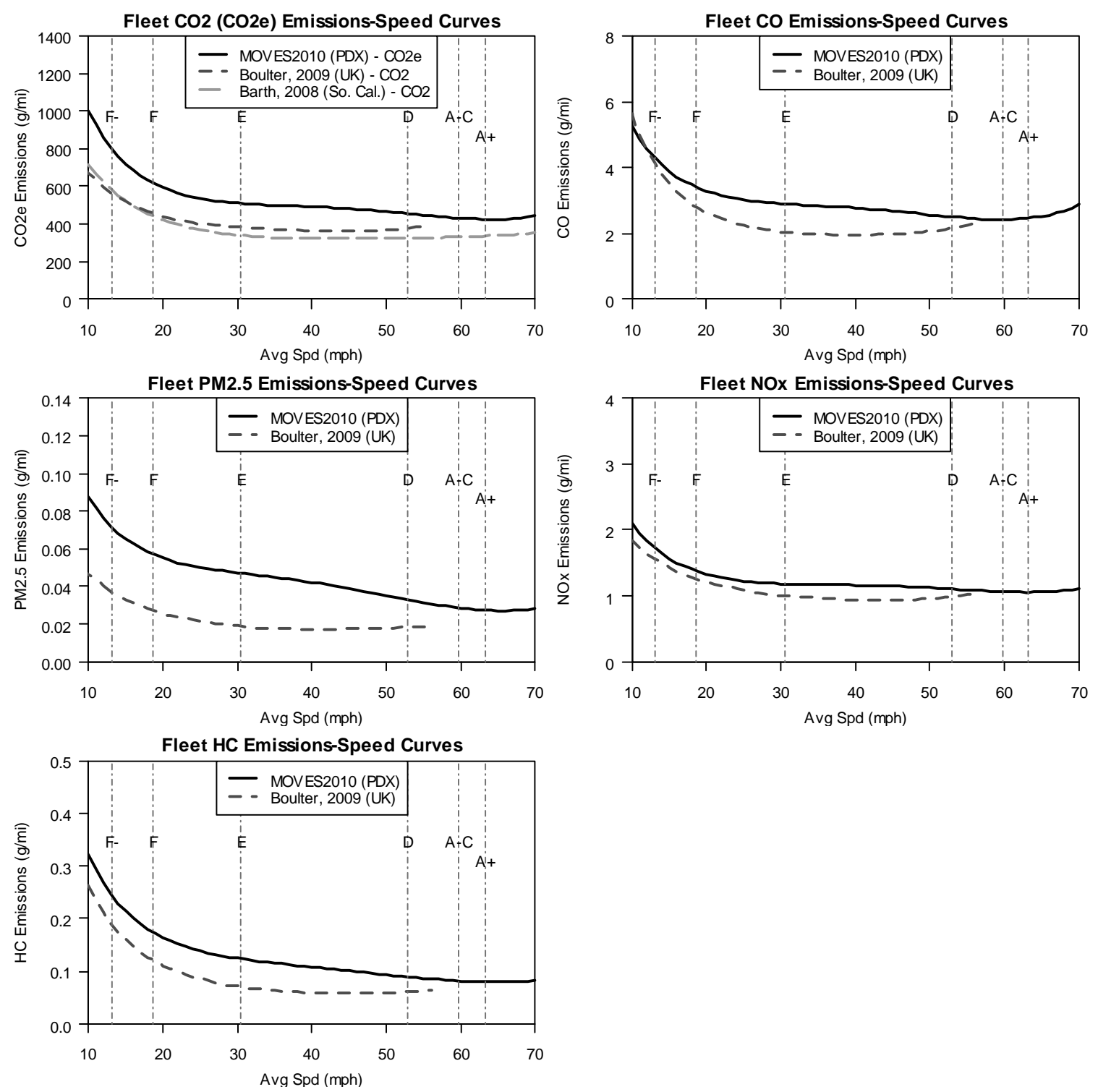

Figure 3. Full-Fleet Freeway Emissions Rates versus Average Speed ( $\overline{\boldsymbol{e}}$ vs. $\bar{v})$, with Freeway LOS

The model sources for the curves in Figure 3 are based on different vehicles, emissions test data, and assumptions, and so it is not surprising that they do not agree on absolute emissions rates. For example, European vehicles (in the Boulter model) have generally more stringent PM controls than U.S. vehicles. The key to observe in these figures is that $\bar{e}$ does not have a monotonic relation with $\bar{v}$, and there are potential 
emissions rate reductions from moderating speeds from both directions. There is also a relatively flat area in the middle of the curve - where sensitivity of $\bar{e}$ to $\bar{v}$ is slight.
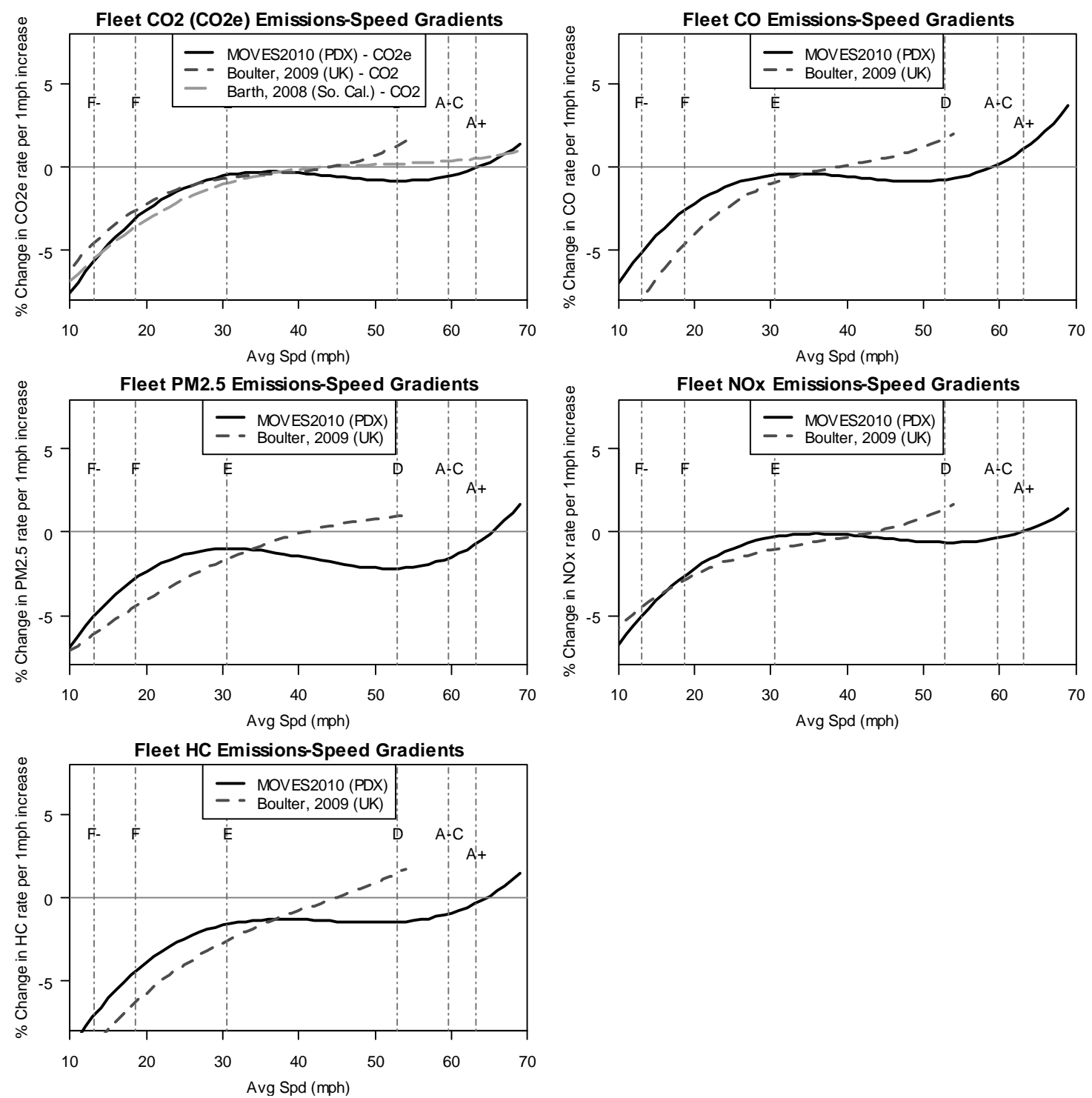

Figure 4. Full-Fleet Freeway Emissions Rate Gradients versus Average Speed, with Freeway LOS

The sensitivity of $\bar{e}$ to $\bar{v}$ is perhaps more easily seen in Figure 4, which shows the ESC gradients versus average travel speed for the same pollutants and models. These are calculated using Equation 16 for $\frac{\partial \bar{e}}{\partial \bar{v}}$, then converting from mass rate changes to 
percentage rate changes for each $1 \mathrm{mph}$ increase in $\bar{v}$. The minimum emissions rate is when the gradient curve crosses the speed (horizontal) axis.

The gradients in Figure 4 have low absolute values from 25-70 mph - meaning speed changes over this range have a small effect on marginal emissions. Increasing speeds above LOS E provides small emissions benefits, and above LOS A can have an emissions-intensifying impact. While the ESC and ESC gradients differ by pollutant, vehicle type, and emissions model, the emissions gradients are consistently small at moderate speeds. As such, few emissions efficiency gains are to be found outside of heavily congested (or extremely high speed) freeway sections. The general agreement among models suggests that these findings apply to other developed countries as well.

The 25-70 mph speed range with low emissions sensitivity is wide enough to encompass most freeway travel. As an example, Figure 5 shows the distribution of freeway VMT in 5-mph speed bins using real-world traffic data from freeways in Portland, Oregon. This figure is based on one month (July 2010) of 5-minute aggregated inductive dual-loop detector data on 15 miles of the I-5 freeway in Portland, Oregon (northbound from milepost 290 to milepost 305). These bounds include roughly 44 million VMT. The data were mined from the PORTAL data archive (http:Ilportal2.its.pdx.edu). The 25-70 mph speed range includes 96\% of all freeway travel, and $81 \%$ of workday peak-hour travel $(5 \mathrm{pm}-6 \mathrm{pm})$. Admittedly, urban areas with more heavy congestion or higher free-flow speeds would have higher percentages of VMT outside of the 25-70 mph range (e.g. see Barth \& Borboonsomsin (2008)). 
I-5, All Hours, July 2010

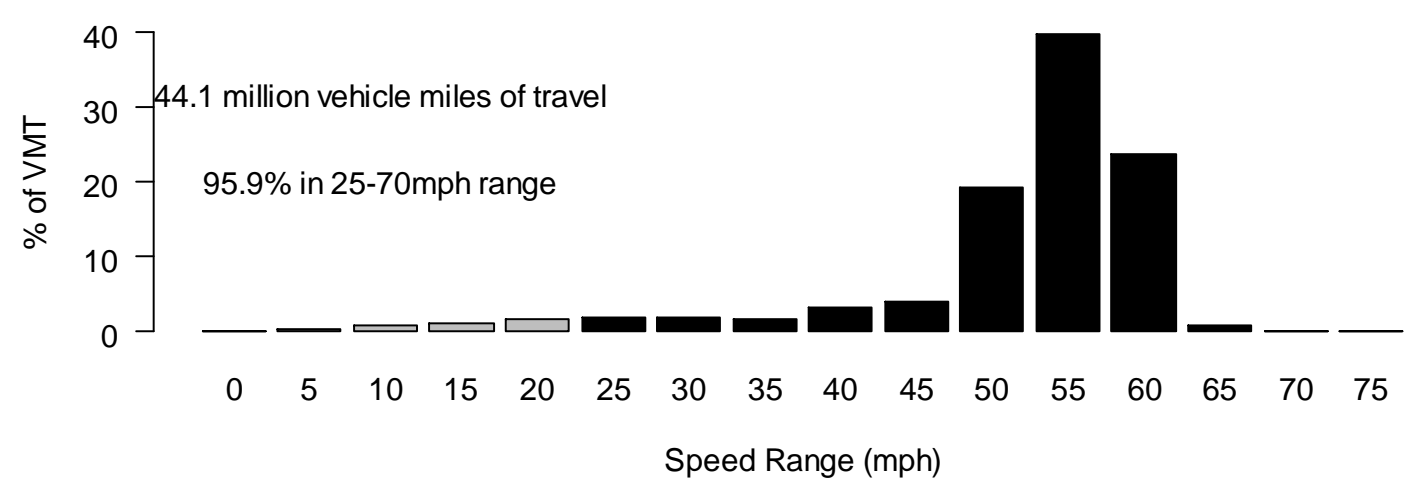

I-5, PM Peak Hour, Non-Holiday Weekdays, July 2010

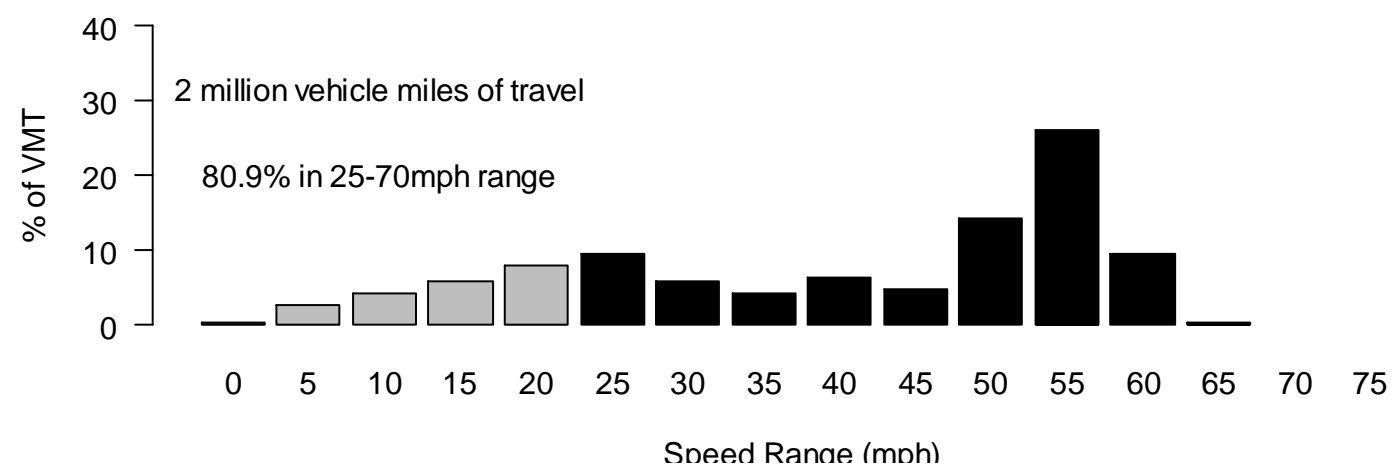

Figure 5. Distribution of VMT using 5-minute average speeds from dual-loop detectors on I-5 northbound in Portland, Oregon

\subsubsection{Arterial versus Freeway Emissions Rates}

The fitted parameters for the MOVES emissions-speed curves (by Equation 6) are shown in Table 5 for the full on-road fleet on arterials for PM peak periods in April, 2010. Figure 6 shows the ESC and ESC gradients that result from the arterial parameters in Table 5. The Boulter ESC and ESC gradients are included in Figure 6 as well, since they are not facility-specific. Although the emissions rates are similar to Figure 3, the lower speed range (expected for arterials) shows decreasing MOVES-modeled emissions rates with increasing speed (negative gradients) over the full speed range. 
Table 5. Full-Fleet MOVES Emissions-Speed Curve Fit Parameters for Arterials

\begin{tabular}{|c|r|r|r|r|r|}
\hline Arterials & \multicolumn{1}{c|}{$\mathbf{C O}_{\mathbf{2}} \mathbf{e}$} & \multicolumn{1}{c|}{$\mathbf{C O}$} & \multicolumn{1}{c|}{$\mathbf{P M}_{\mathbf{2 . 5}}$} & \multicolumn{1}{c|}{$\mathbf{N O}_{\mathbf{x}}$} & \multicolumn{1}{c|}{ HC } \\
\hline$a_{0}$ & 8.161 & 2.772 & -1.277 & 1.852 & 0.2974 \\
\hline$a_{1}$ & -0.1735 & -0.1378 & -0.1618 & -0.1554 & -0.1960 \\
\hline$a_{2}$ & 0.005899 & 0.004602 & 0.005876 & 0.005390 & 0.006389 \\
\hline$a_{3}$ & $-8.937 \mathrm{E}-05$ & $-7.356 \mathrm{E}-05$ & $-9.883 \mathrm{E}-05$ & $-8.239 \mathrm{E}-05$ & $-9.841 \mathrm{E}-05$ \\
\hline$a_{4}$ & $4.929 \mathrm{E}-07$ & $4.435 \mathrm{E}-07$ & $5.896 \mathrm{E}-07$ & $4.572 \mathrm{E}-07$ & $5.576 \mathrm{E}-07$ \\
\hline
\end{tabular}

Figure 7 compares the freeway and arterial full-fleet ESC gradients from the MOVES model using the fitted parameters, $a_{i}$, shown in Table 4 and Table 5. The differences in the curves arise because of differing driving patterns on each facility: different combinations of vehicle operating modes can result in the same average travel speed. All other factors are the same between the curves (fleet composition, vehicles, weather, fuel, etc.). The shapes are quite similar, although the arterial emissions rates are slightly more sensitive to speed. This means that the potential for emissions rate reductions by increasing average speeds is greater on arterials than on freeways. This is particularly true considering the speed range differences; a moderately congested arterial has a lower average speed than a moderately congested freeway. 

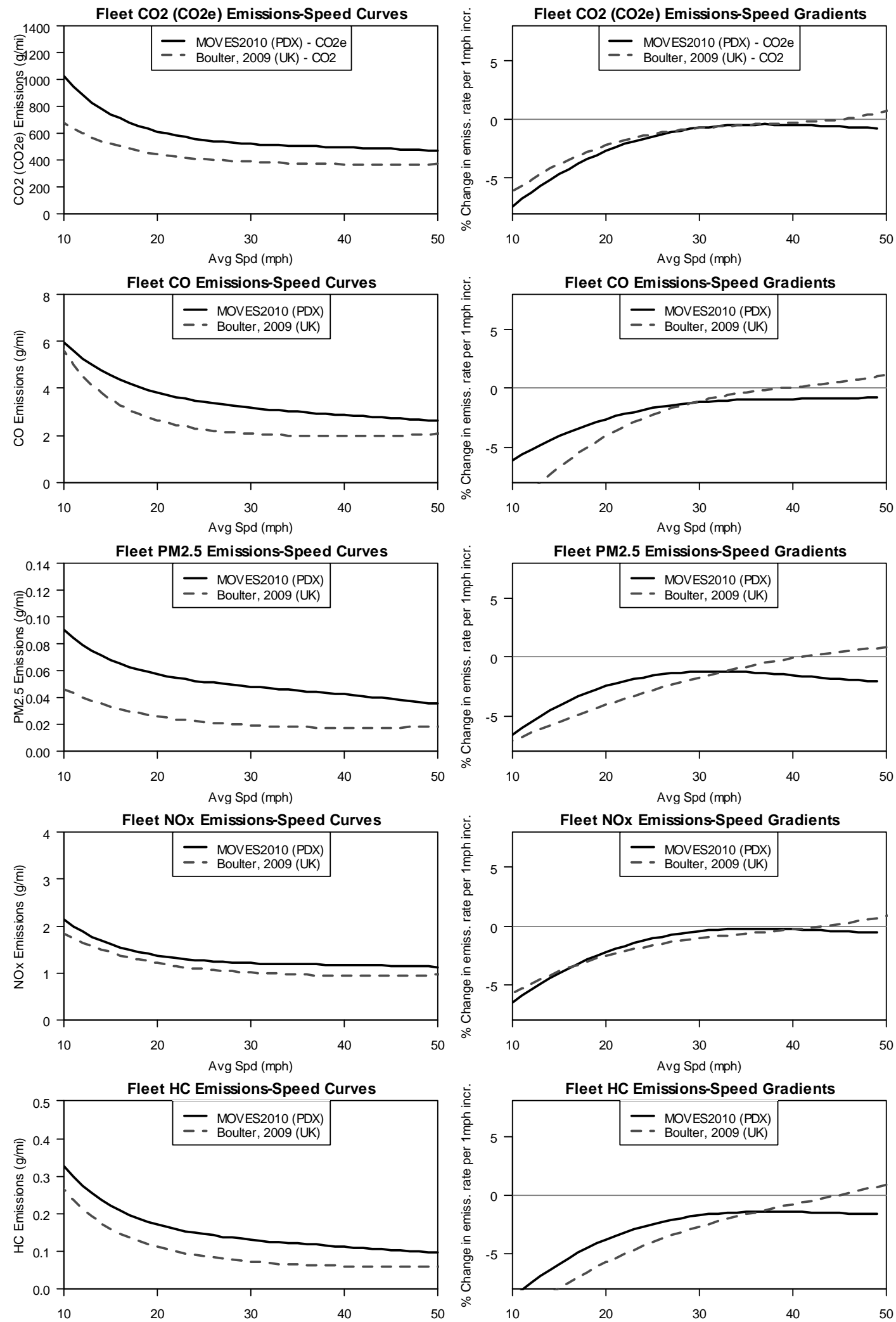

Figure 6. ESC and ESC Gradients for Arterial Emissions 

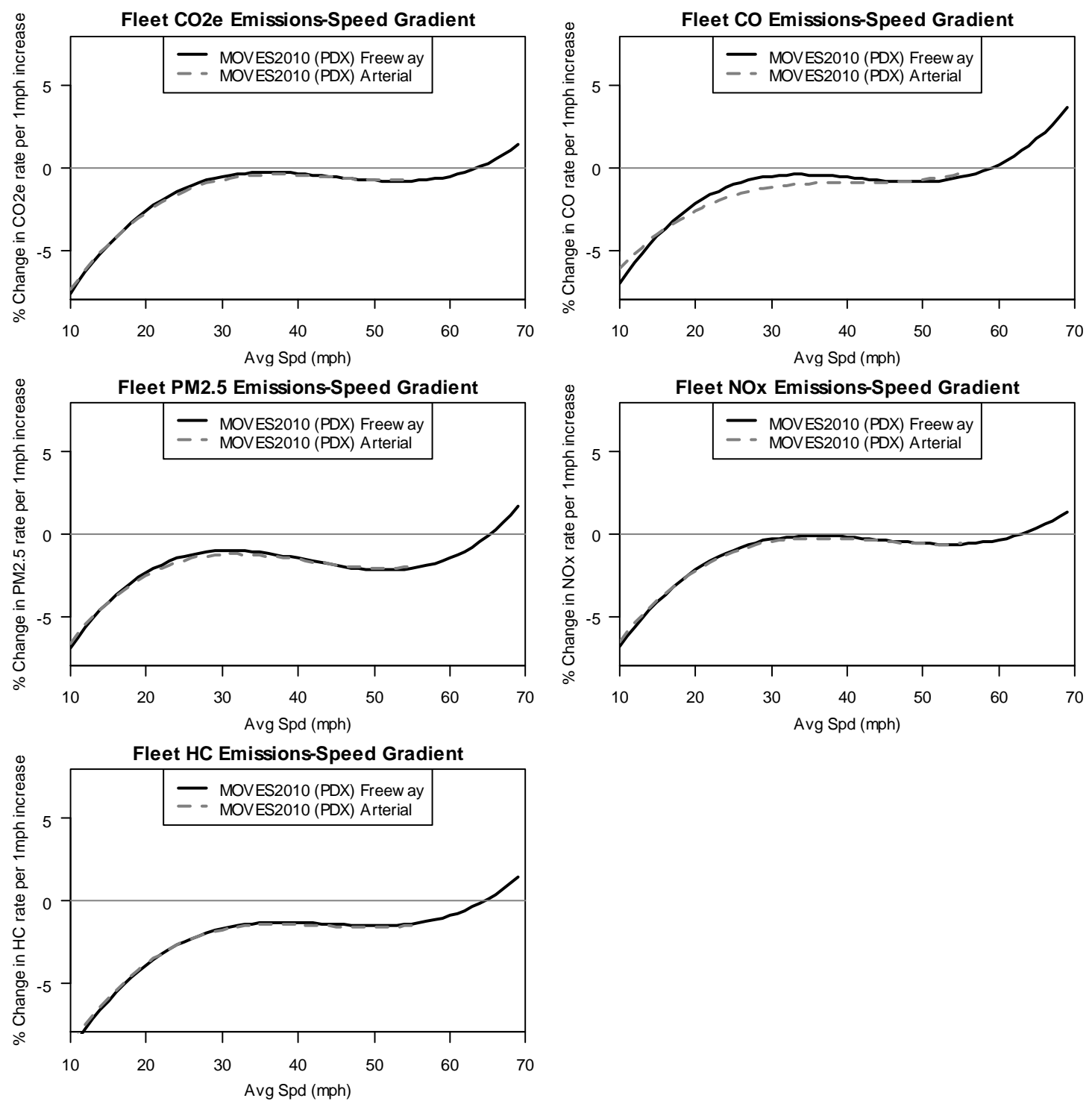

Figure 7. Comparison of freeway and arterial emissions-speed gradients

We can examine the facility-specific driving schedules which underlie the average-speed emissions models (see Section 2.3.2.2) for an explanation of why the arterial and freeway ESC gradients in Figure 7 are different. Figure 8 shows the distributions of second-by-second speeds and accelerations for two different driving schedules mined from the MOVES drive schedule library (used for average-speed emissions estimates as described in Section 2.3.2.2). The top histogram is for a drive 
schedule representing LOS F conditions on a freeway facility for LD vehicles, with an average speed of $21 \mathrm{mph}$ (MOVES drive schedule ID 1021). The bottom histogram shows a drive schedule with a slightly higher average speed, $25 \mathrm{mph}$, for LOS C conditions on an arterial facility for LD vehicles (MOVES drive schedule ID 1030). Although the overall average speeds are similar, the freeway drive schedule contains more low-speed driving in the 5-25 mph range, while the arterial drive schedule contains more idling and mid-speed driving (around 25-40 mph). Additionally, the two drive schedules have different distributions of second-by-second accelerations, with more heavy accelerations and decelerations for the arterial drive schedule. These second-bysecond speed and acceleration differences between facility-specific drive schedules with similar average speeds result in different emissions rate estimates for a given average speed because they generate different engine loading estimates during emissions modeling. 

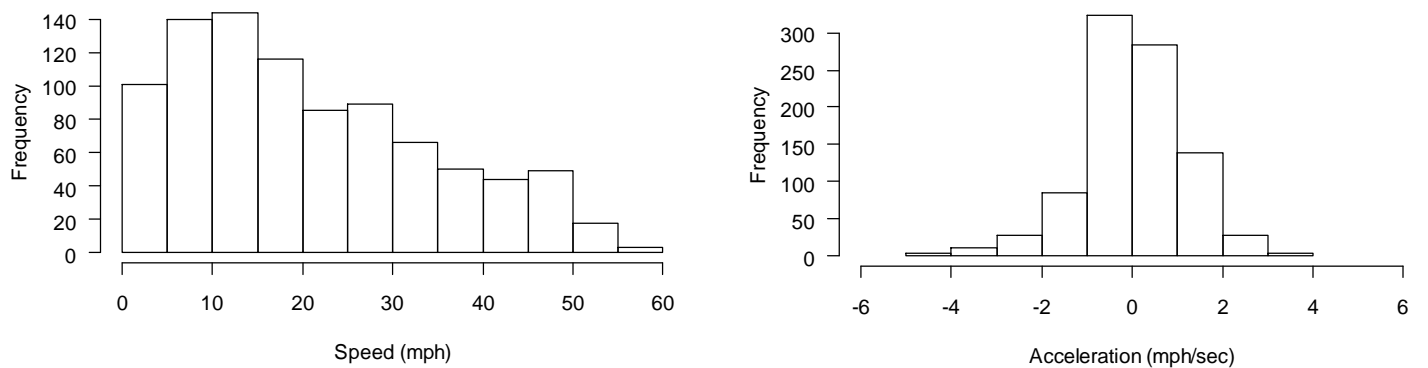

Arterial Drive Schuedule, LOS C, Avg. Speed $25 \mathrm{mph}$ Arterial Drive Schuedule, LOS C, Avg. Speed $25 \mathrm{mph}$
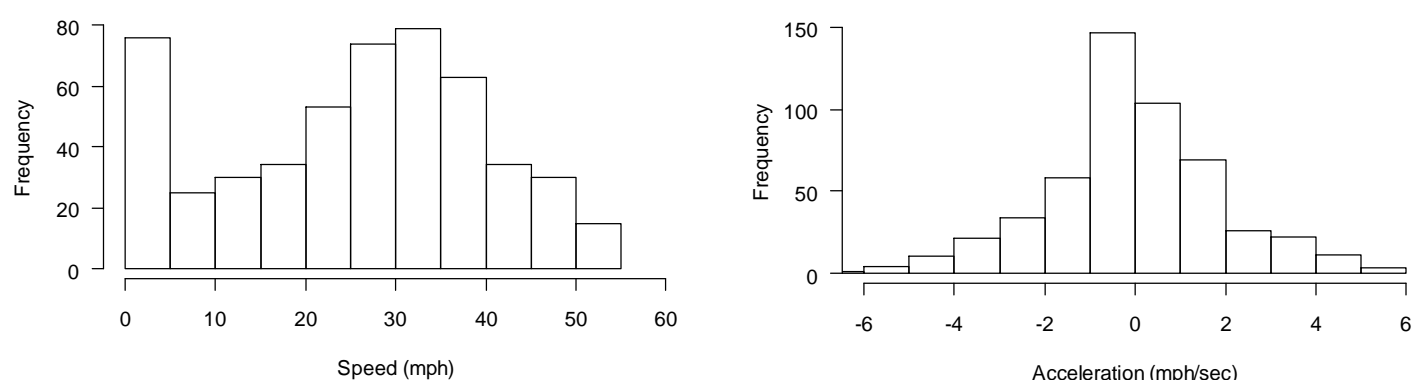

Figure 8. Comparison of Speed and Acceleration Distributions for Freeway and Arterial Drive Schedules with Similar Average Speeds

By various models and for various pollutants, the consistent pattern appears of stagnant emissions rates per vehicle-mile over a wide range of moderate speeds. At the more extreme speeds (below 25 and above $70 \mathrm{mph}$ ) travel efficiency degrades rapidly. A comparison of ESC for different seasons and hours shows no notable difference. This is expected, as we have modeled running exhaust emissions and seasonal/hourly variations in emissions rates are due to meteorological differences which mostly affect evaporative emissions. A final note on the sensitivity of the ESC and ESC gradients is that they are based on archetypal driving patterns and average-speed emissions modeling. Drive schedules representing different driver, roadway, or vehicle characteristics will produce different ESC (see Section 2.3.2.2). Changes in microscopic traffic characteristics over time (behavioral, technological, or operational) will also affect the shapes of the ESC. 


\subsection{Temporal Emissions Rates (per vehicle-hour)}

The ESC in Section 5.1 describe the relationships between average travel speed $\bar{v}$ and average emissions rates normalized to travel distance, $\bar{e}$. In this section we look at the relationship between average travel speed $\bar{v}$ and full-fleet average emissions rates normalized to travel time, $\bar{e}^{\prime}$, as calculated from Equation 7. We use the same fitted parameters $a_{i}$ as in Section 5.1, shown in Table 4. With $\bar{v}$ in mph, $\bar{e}^{\prime}$ is then in grams per vehicle-hour.

Temporal marginal emissions rates versus average speed curves describe how the average travel speed affects a single vehicle's emissions rate per hour of operation. For assessing long-term total emissions characteristics, temporal rate curves would be indicative of the total emissions-speed relationship if travel demand were fully elastic with travel time - i.e. total travel time were fixed. This scenario has been suggested by Metz (2008), who claims that in the long run average travelers adjust their travel behavior by modifying their access choices while maintaining a fairly constant travel time budget. Such an approach differs greatly from the application of spatial emissions rates (as found in Section 5.1) for total emissions-speed relationships, which implies fixed travel distance insensitive to travel time constraints (i.e. inelastic demand).

An illustrative comparison of marginal freeway fleet $\mathrm{CO}_{2}$ e emissions rates (both $\bar{e}$ and $\bar{e}^{\prime}$ versus $\bar{v}$ ) is shown in Figure 9 for Portland 2010 in grams per vehicle-minute and grams per vehicle-mile. These curves meet at $60 \mathrm{mph}$ where the travel rate is 1 minute per mile. At low speeds the curves display diverging behavior. Per unit distance, the spatial emissions rates $\bar{e}$ increase at lower speeds because of inefficient driving and longer 
operating time. Per unit time, the temporal emissions rates $\bar{e}^{\prime}$ decrease at lower speeds because of lower engine loads.

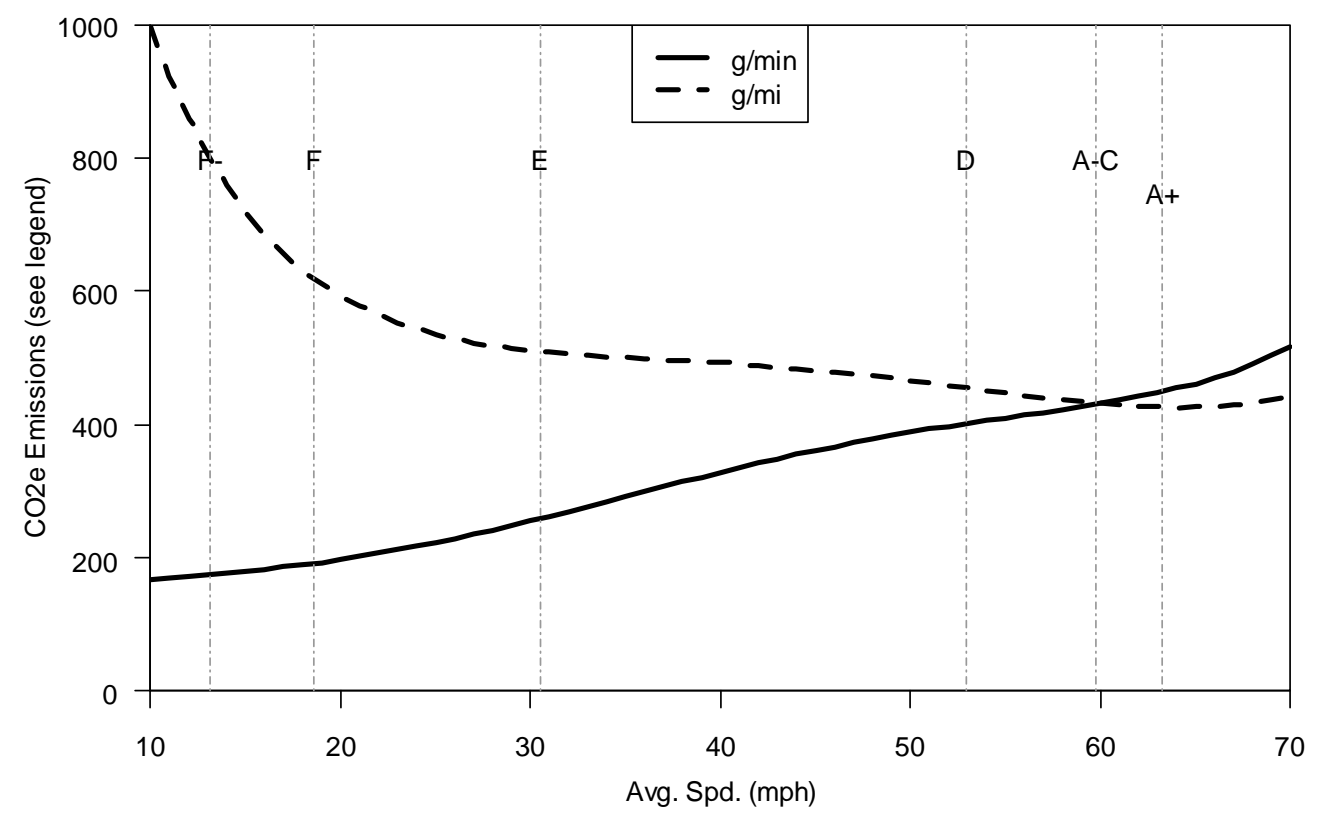

\section{Figure 9. Fleet $\mathrm{CO}_{2} \mathrm{e}$ emissions rates per mile and per minute, with freeway LOS}

From a long-term perspective, the low-speed slope of total emissions as a function of average speed depends on the relative stability of travel distance and travel time. If total travel distance is fixed (perfectly inelastic), total emissions increase with lower speeds similarly to spatial emissions rates. If adjusting for shorter travel distances to maintain travel time (perfectly elastic demand), total emissions decrease at lower speeds similarly to temporal emissions rates. The long-term reality of total emissions is somewhere in between the perfectly inelastic and elastic demand projections. If we assume that in the long-run travelers are not fixed to an absolute travel distance or time, but make trade-offs depending on the utility of each, then the most representative shape is somewhere in between these curves. As such, the long-term emissions inefficiencies of 
low travel speeds are not as great as they appear to be from the spatial emissions rate curves in Section 5.1.

\subsection{Emissions Rates as a Function of Volume}

For spatial marginal emissions rates as a function of traffic volume, $\bar{e}\left(q^{\prime}\right)$, we estimated the parameters in Equation 9 using values of $\bar{e}$ from Equation 8 with the MOVES-modeled parameters above for $a_{i}, t_{o}=1 \mathrm{mile} / \mathrm{minute}(60 \mathrm{mph}$ free-flow speed), $c=2,200$ pcphpl (passenger cars per hour, per lane), $\alpha=0.15$ and $\beta=7$. Minimizing the sum of square error with non-negative parameters using $q^{\prime}$ as the independent variable from 0 to 3,630 pcphpl, we generate the parameter estimates shown in Table 6. This range for $q^{\prime}$ was selected because it generates travel speed estimates from 10 to 60 mph using the BPR function. Results for $\mathrm{CO}_{2} \mathrm{e}$ (MOVES) and $\mathrm{CO}_{2}$ (Barth) are illustrated in Figure 10.

Table 6. Fitted Emissions Parameters from Equation 9

\begin{tabular}{|c|l|l|l|l|l|l|}
\hline Parameter & $\begin{array}{l}\mathbf{C O}_{\mathbf{2}} \mathbf{e} \\
\text {-MOVES }\end{array}$ & $\begin{array}{l}\mathbf{C O}_{2} \\
\text {-Barth }\end{array}$ & $\begin{array}{l}\mathbf{C O} \\
\text {-MOVES }\end{array}$ & $\begin{array}{l}\text { PM } \\
\text {-MOVES }\end{array}$ & $\begin{array}{l}\mathbf{N O}_{\mathbf{x}} \\
\text {-MOVES }\end{array}$ & $\begin{array}{l}\text { HC } \\
\text {-MOVES }\end{array}$ \\
\hline$b_{0}$ & 423 & 327 & 2.37 & 0.0248 & 1.05 & 0.0761 \\
\hline$b_{1}$ & 27.0 & 0.000 & 0.168 & 0.0105 & 0.0465 & 0.0163 \\
\hline$b_{2}$ & 3.55 & 1.67 & 0.0176 & 0.000331 & 0.00398 & 0.00153 \\
\hline$m$ & 9.98 & 10.1 & 10.0 & 9.97 & 10.0 & 10.0 \\
\hline $\mathrm{R}^{2}$ & 0.995 & 0.990 & 0.993 & 0.968 & 0.994 & 0.995 \\
\hline
\end{tabular}

The fit for all pollutants is good, with $\mathrm{R}^{2}$ values above 0.96 . The parameter $m$ is about 10 for all pollutants, reflecting a similar shape to the emissions rate versus demand volume curve. Comparing the MOVES and Barth models for $\mathrm{CO}_{2} \mathrm{e}$ and $\mathrm{CO}_{2}$, respectively, the proposed formulation works for both despite the different fleet 
compositions and emissions model data. The difference in magnitude is to be expected, as the MOVES model includes heavy vehicles while the Barth model does not.

Looking at Figure 10, the $\bar{e}\left(q^{\prime}\right)$ formulation in Equation 9 smoothes out the MOVES curve around $q^{\prime}=2,500 \mathrm{pcphpl}$. Since the fitted parameters are non-negative (to create a concave form for Equation 9), the decrease in emissions rates around $q^{\prime}=2,500 \mathrm{pcphpl}$ in the Barth model is not captured by this formulation. From Table 6, though, only the Barth $\mathrm{CO}_{2}$ model is constrained by non-negativity. The decrease in emissions rate occurs because the assumed free-flow speed is above the optimal speed in the Barth Model. The non-decreasing emissions rate formulation will not reflect this initial efficiency gain for high free-flow (or low optimal) speeds. Still, these results show that Equation 9 is a good approximation of $\bar{e}\left(q^{\prime}\right)$ for certain free-flow speed and emissions-minimizing speed conditions (particularly when the later exceeds the former). These curves can be used for traffic modeling which requires simplified and integrated emissions and volume estimation, such as emissions-minimizing traffic flow optimization (e.g. Bigazzi \& Figliozzi (2011), Sugawara and Niemeier (2002)). 


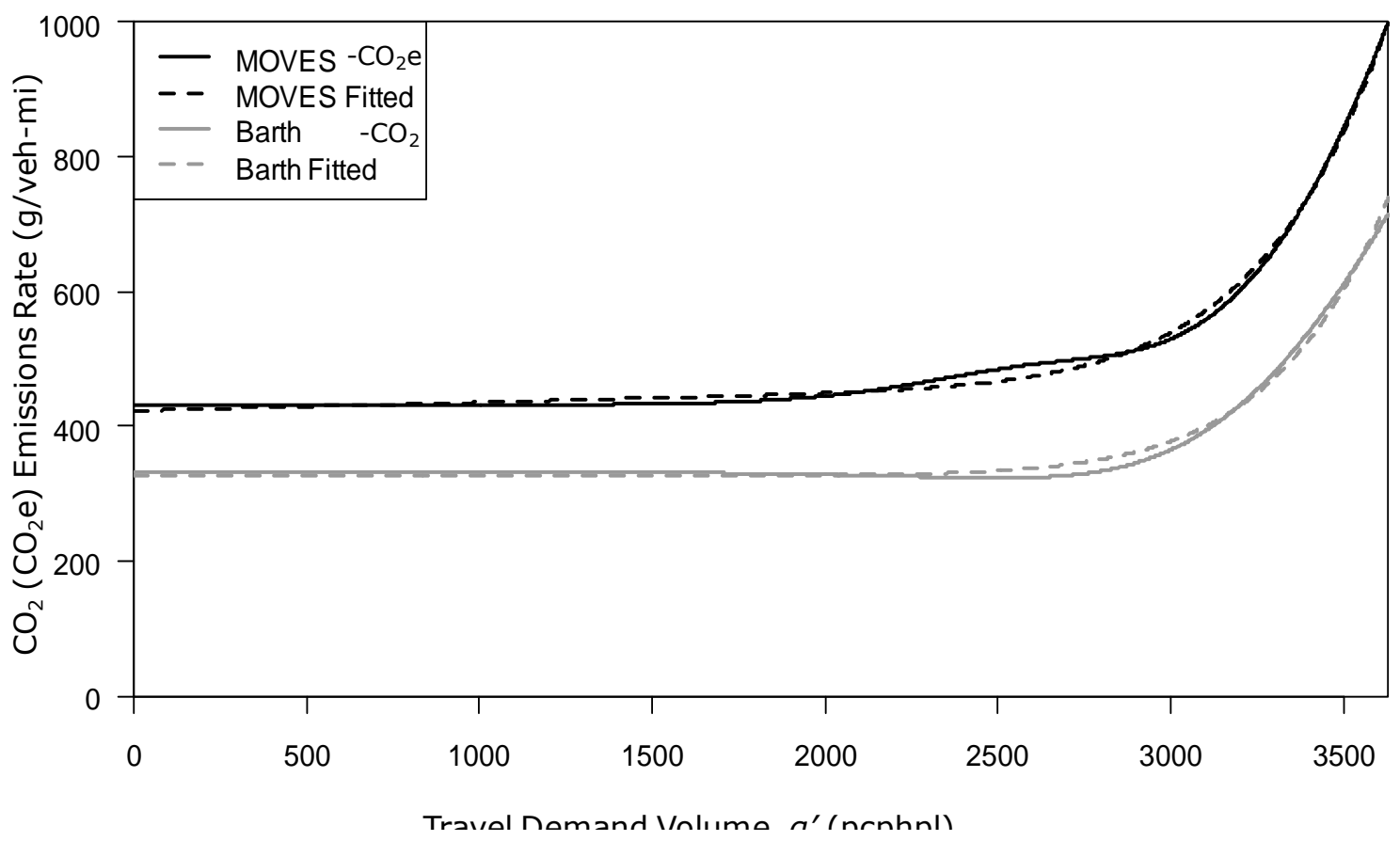

Figure 10. $\mathrm{CO}_{2}$ Emissions Fits for MOVES (Black) and Barth (Grey) Models by

\section{Equation 9}

\subsection{Total Emissions}

Relationships between total emissions and traffic speed can assist with macroscopic mitigation strategy development and assessment, targeting both vehicle emissions and congestion. While the figures in Section 5.1 demonstrate emissions rate benefits of increasing congested vehicle speeds, the impacts of varying travel distances illustrated by Figure 9 show that congestion mitigation strategies must also assess traffic volume when estimating total emissions effects of speed improvements. Increasing congested travel speeds will often reduce the average vehicle's spatial marginal emissions rate, but it will also induce more travel. 


\subsubsection{Total Emissions and Demand Volume}

The total on-road emissions, $E$, from a given demand volume of vehicles, $q$, is calculated using Equation 10 and the average emissions rates, $\bar{e}$, from Section 5.1. Figure 11 shows $\mathrm{CO}_{2} \mathrm{e}$ total emissions, $E$, as shading on the $\bar{v}$ versus $q^{\prime}$ plane (assuming no PCE adjustment, $q^{\prime}=q$ ). The curve on Figure 11 is the theoretical relationship between demand volume and average speed from the BPR Equation (1). The ESC shown in Section 5.1 can be seen as vertical slices of the shading contours, magnified by flow rate (since $E=q \cdot \bar{e})$.

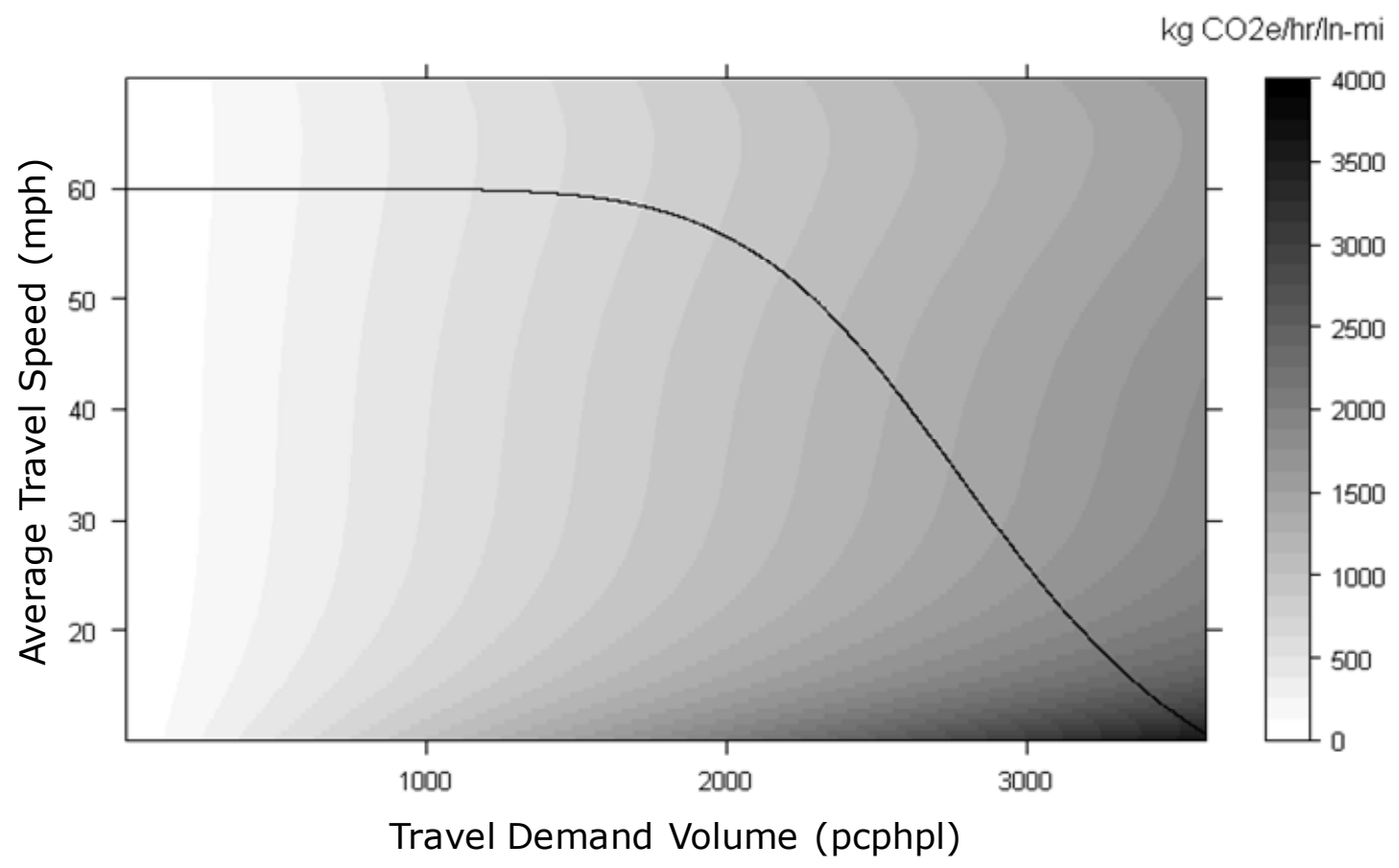

Figure 11. Total Emissions $(E)$ as Shading on the Speed $(\bar{v})$ versus Effective Demand Volume $\left(q^{\prime}\right)$ Plane, with BPR Curve

Following the BPR curve in Figure 11 from left to right, we see that total emissions continually increase with demand volume. This occurs not only because $E$ is linearly proportional to $q$, but because increasing $q$ decreases $\bar{v}$ - which, in turn, 
increases $\bar{e}$ (see Figure 3 ). The speed impact on total emissions is particularly

pronounced at low speeds ( $\bar{v}<25 \mathrm{mph}, q>3,000 \mathrm{pcphpl})$ where $\bar{e}$ increases rapidly with decreasing $\bar{v}$.

\subsubsection{Total Emissions and Vehicle Throughput}

Using demand flow $q$ to calculate total emissions $E$ accounts for vehicles queued or delayed upstream during congestion. But during heavy congestion when $q^{\prime}>c$, actual vehicle throughput on the section of interest will be less than $q$ on a limited temporal scale (May, 1989). In this case some of the emissions on the road section of interest will be displaced upstream or delayed until the next time period, though the total emissions will be the same. For a demand volume $q$, let us define the actual vehicle throughput at a specific location for a specific time period as $\hat{q}$, in the same units as $q$.

We can illustrate the displaced emissions effect using total emissions estimates (based on $E=\hat{q} \cdot \bar{e}$ ) with observed traffic data. Figure 12 presents total emissions $(E)$ contours in kg per hour per lane-mile of roadway as lines and shadings on the traffic speed ( $\bar{v}$ in mph) versus vehicle throughput ( $\hat{q}$ in vphpl - vehicles per hour, per lane) plane. The contour lines are iso-emissions lines. In addition, Figure 12 shows 5-minute aggregated traffic states from all 24 hours of January 21, 2010 on I-5 northbound in Portland, Oregon (as circles). These traffic data were collected from 15 inductive dualloop detector stations on the freeway (between mileposts 290 and 305) and mined from the PORTAL transportation data archive at Portland State University (http:Ilportal.its.pdx.edu). The 5-minute aggregation was selected because it has been shown elsewhere to best approximate average freeway travel speeds (Bigazzi, Siri, \& 
Bertini, 2010; Wang \& Liu, 2005) and it is short enough that $q^{\prime}>c$ for some time periods on this busy freeway. The observed speeds are used to estimate $\bar{e}$ from the MOVES-modeled parameters above.
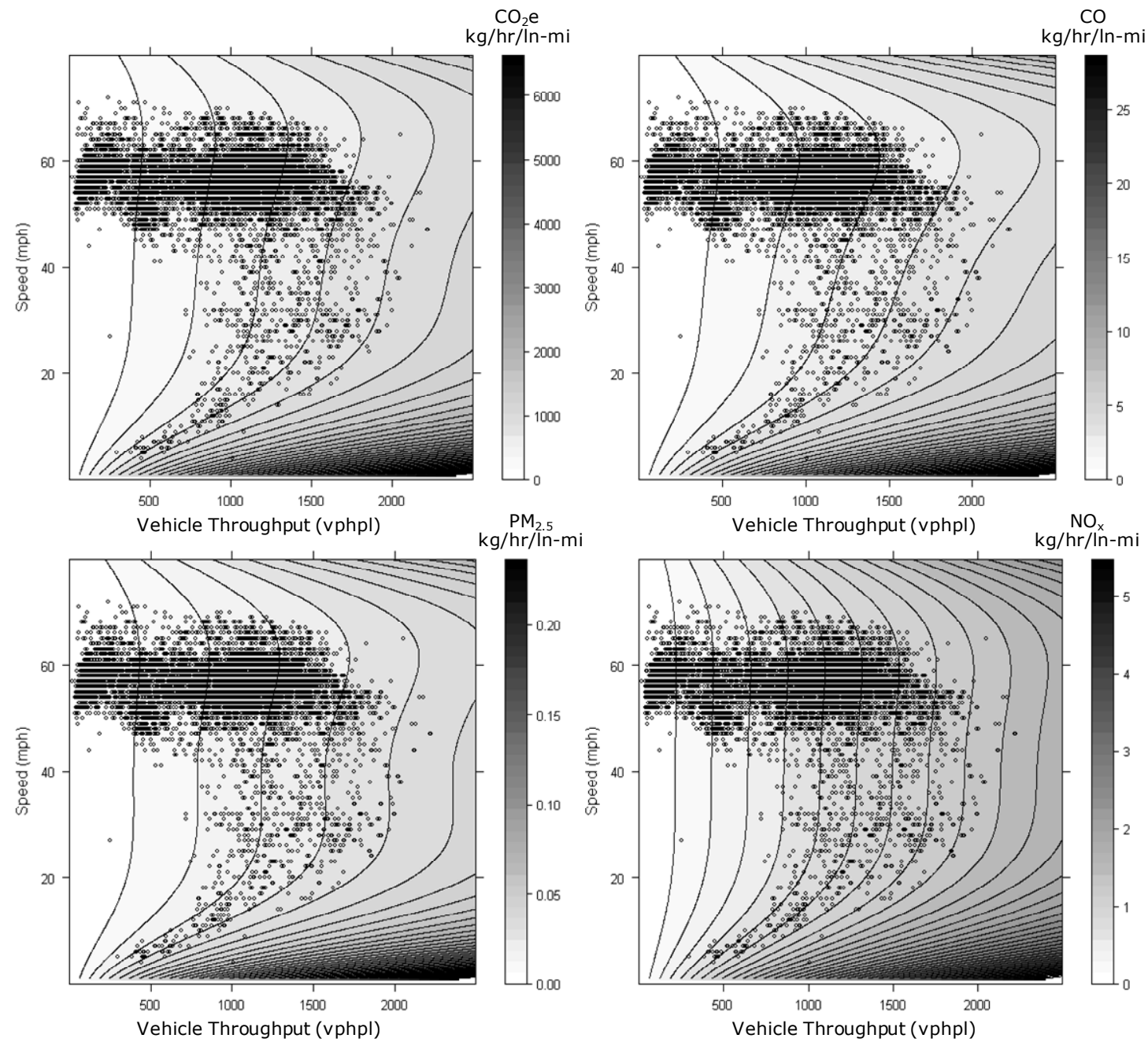

Figure 12. Total Emissions $(E)$ as Shading and Contours on the Vehicle Speed $(\bar{v})$ versus Vehicle Throughput $(\widehat{\boldsymbol{q}})$ Plane, with Observed Traffic States

From Figure 12 we see that no observed 5-minute interval saw $\hat{q}>2,300$ vphpl. Congested time intervals with average speeds from 20 to $45 \mathrm{mph}$ had throughput mostly in the range $1,000 \leq \hat{q} \leq 2,000$ vphpl. Heavier congestion with $\bar{v}<20 \mathrm{mph}$ had 
decreasing $\hat{q}$ down to under $500 \mathrm{vphpl}$. The measured traffic states display a great deal of variance - partly because they were measured at several locations on the corridor.

As expected from Figure 4, for a wide range of average speeds from 25 to $70 \mathrm{mph}$ the effect of $\bar{v}$ on $E$ is negligible, and $E$ for different time intervals varies mostly with $\widehat{q}$. This is observed through the vertical orientation of the contour lines. Uncongested time intervals from 50 to $70 \mathrm{mph}$ have a particularly wide range of $\hat{q}$ (and $E$ ). For heavy congestion $(\bar{v}<20 \mathrm{mph})$, the decreasing $\hat{q}$ with lower $\bar{v}$ offsets increasing $\bar{e}$, resulting in similar $E$ during heavy congestion as during more moderate congestion or high-volume uncongested conditions.

Figure 12 presents a different picture of congestion effects on $E$ than Figure 11, where $E$ and $\bar{v}$ have monotonic relationships with $q$. This is because $\hat{q}$ fails to account for displaced vehicle emissions during congestion. The increasing $E$ expected at low $\bar{v}$ from Figure 11 is real, but it is displaced to another time interval or an upstream section of roadway in Figure 12. The importance of this difference is one of scope of concern. For global pollutants like greenhouse gases the location of emissions is unimportant and $q$ is most representative. For short-lived local pollutants and analyses of limited spatial and temporal scope, $\hat{q}$ may be more relevant, depending on the time scale. However, an analysis of congestion-emissions relationships should be cautious in using $\hat{q}$ to estimate total emissions since displaced emissions due to congestion are not included. Since $q$ is a more comprehensive measure, for the remainder of this analysis we will consider only $q$ in calculating $E$. 


\subsubsection{Total Emissions Sensitivity to Speed and Volume}

Figure 13 shows the elasticity of emissions rates to speed $\varepsilon_{\bar{e}}^{\bar{v}}$, computed by Equation 17. Referring to Figure 11 and Figure $12, \varepsilon \overline{\bar{v}}$ is the vertical gradient in $E(q, \bar{v})-$ the total emissions on the traffic speed-flow plane - expressed as elasticity (the percent change in total emissions $E$ with each percent change speed $\bar{v}$ at a fixed flow $q$ ). The horizontal gradient of $E(\bar{v}, q)$, expressed as an elasticity (the percent change in total emissions $E$ with each percent change in flow $q$ at a fixed speed $\bar{v}$ ), is 1 . Thus absolute values of $\varepsilon_{\bar{e}}^{\bar{v}}$ less the 1 in Figure 13, $\left|\varepsilon_{\bar{e}}^{\bar{v}}\right|<1$, reflect less sensitivity to speed than to flow.

Here, $\left|\varepsilon_{\bar{e}}^{\bar{v}}\right|<1$ for nearly all traffic states for all five pollutants considered.

Notable exceptions are the high sensitivity of $\mathrm{CO}$ to high travel speeds (above about 65 $\mathrm{mph}$ ), the high sensitivity of $\mathrm{PM}_{2.5}$ to traffic speeds in the 50-60 mph range, and the sensitivity of $\mathrm{HC}$ to very low travel speeds (below about $15 \mathrm{mph}$ ).

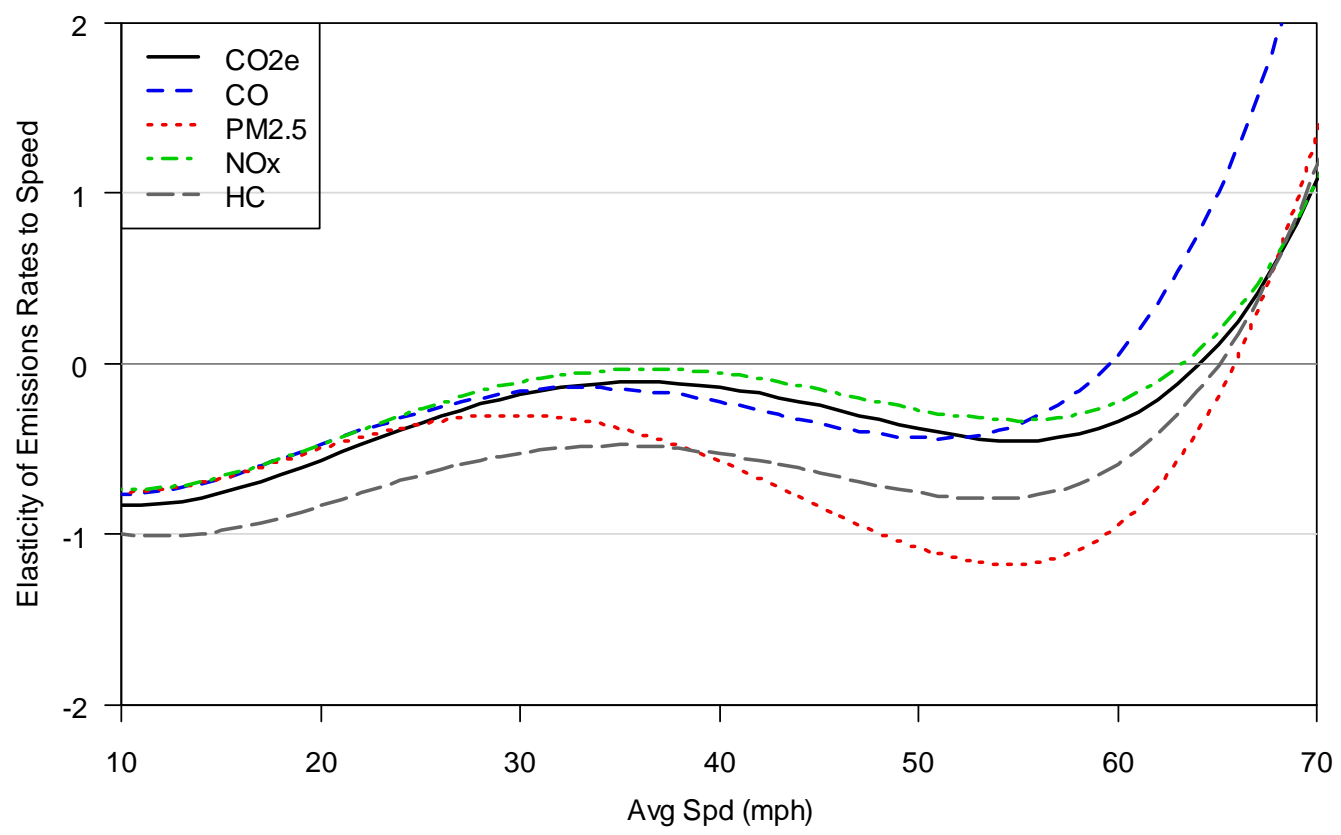

Figure 13. Elasticity of Emissions Rates to Speed, $\varepsilon_{\bar{e}}^{\bar{v}}$, by MOVES Model 


\subsection{Total Emissions Elasticity to Speed - Including Variable Traffic Volume}

In Section 5.4 we looked at how average speed affects total emissions from a given volume or flow of vehicles. This section describes the impacts of average travel speed on total emissions including variable travel demand volumes with travel speed. We define the elasticity of travel demand volume to average travel speed for the full fleet as

$\eta_{q}^{\bar{v}}$. The elasticity of total emissions to changes in speed, $\varepsilon_{E}^{\bar{v}}$, is described by the equations of Section 4.6. By looking at how changing speeds impact the traffic volume and total emissions, we are essentially assessing the emissions impacts of capacity-based congestion mitigation, described in Section 2.6.1.

The elasticity of total $\mathrm{CO}_{2} \mathrm{e}$ emissions to changes in speed, $\varepsilon_{E}^{\bar{v}}$, calculated by Equation 27, is shown in Figure 14for a MOVES-modeled composite freeway fleet with varying average speed, $\bar{v}$, and demand elasticity to speed, $\eta_{q}^{\bar{v}}$. Total $\mathrm{CO}_{2} \mathrm{e}$ emissions increase most with speed changes at very high speeds (because the marginal emissions rates, $\bar{e}$, increase quickly) and at high demand elasticity to speed (because of traffic volume, $q$, increases). The total emissions elasticity $\varepsilon_{E}^{\bar{v}}$ is negative at very low demand elasticities with moderate speeds (because of minimal volume increases) or at moderate elasticities with very low speeds (because of large emissions rate reductions). The zerovalued contour line in Figure 14 shows the break-even conditions from an emissions perspective described in Section 4.7. Combinations of $\bar{v}$ and $\eta_{q}^{\bar{v}}$ above this break-even line will increase total emissions with a speed increase while combinations below this line will decrease total emissions with a speed increase. 


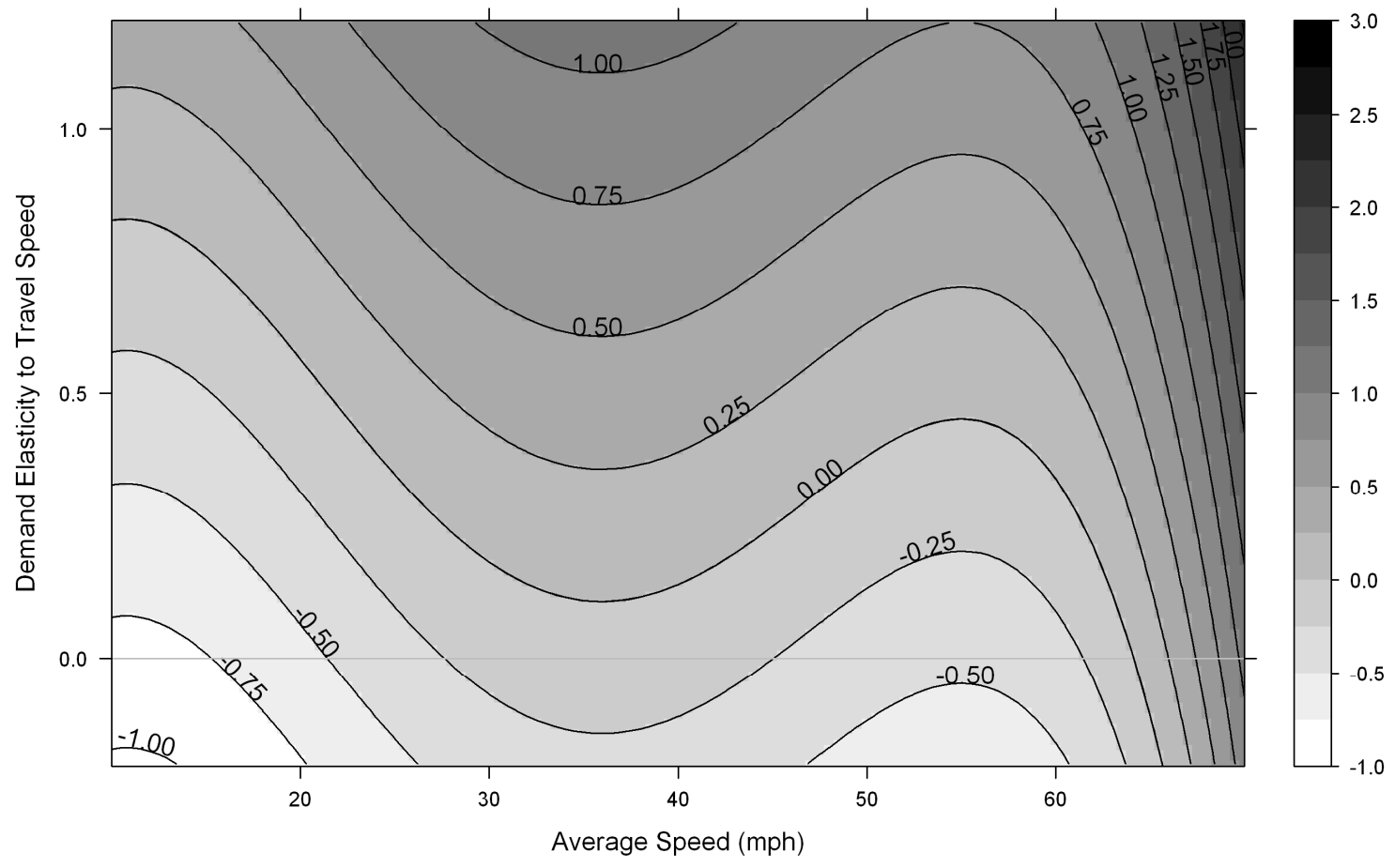

Figure 14. Elasticity of Total $\mathrm{CO}_{2} \mathrm{e}$ Emissions to Changes in Speed, $\varepsilon_{E}^{\bar{v}}$, as Shadings and Contours on the $\eta_{q}^{\bar{v}}$ versus $\bar{v}$ Plane

Figure 15 repeats Figure 14 for the other four pollutants modeled. The relationships are generally similar for $\mathrm{CO}$ and $\mathrm{NO}_{x}$ as compared to $\mathrm{CO}_{2} \mathrm{e}$, although $\mathrm{CO}$ has higher positive $\varepsilon_{E}^{\bar{v}}$ for speeds above $60 \mathrm{mph} . \mathrm{PM}_{2.5}$ and $\mathrm{HC}$ both have generally lower $\varepsilon_{E}^{\bar{v}}$ than $\mathrm{CO}_{2} \mathrm{e}$. This is particularly true for $\mathrm{PM}_{2.5}$ at speeds between 40 and $60 \mathrm{mph}$. This difference indicates that, compared to $\mathrm{CO}_{2} \mathrm{e}$, total emissions of $\mathrm{PM}_{2.5}$ and $\mathrm{HC}$ are more likely to decrease with speed increases or increase with speed decreases. 

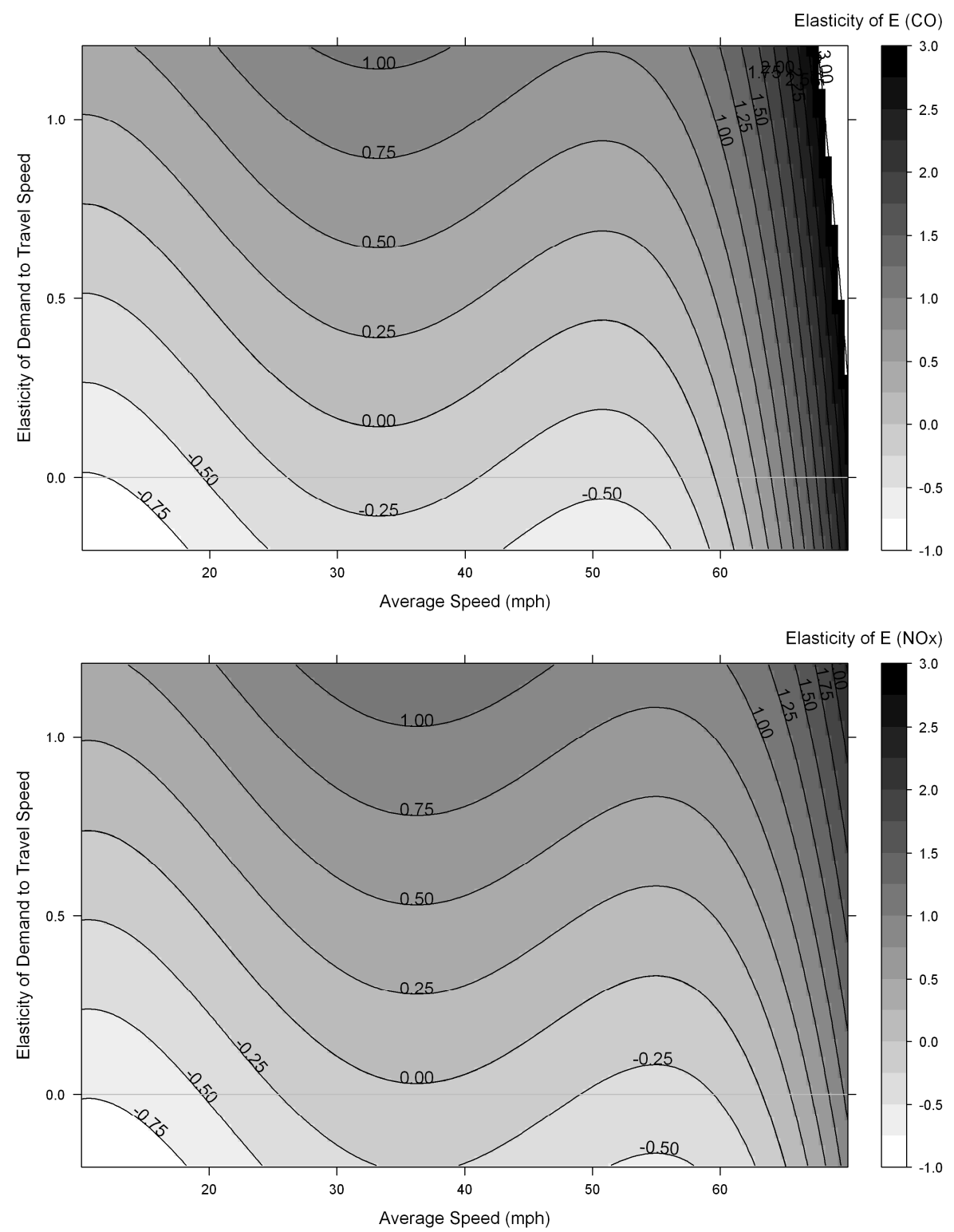

Figure 15. (Part I) Elasticity of Total Emissions to Speed, $\varepsilon_{E}^{\bar{v}}$, for CO and NOx, as Shadings and Contours on the $\eta_{q}^{\bar{v}}$ versus $\bar{v}$ Plane 

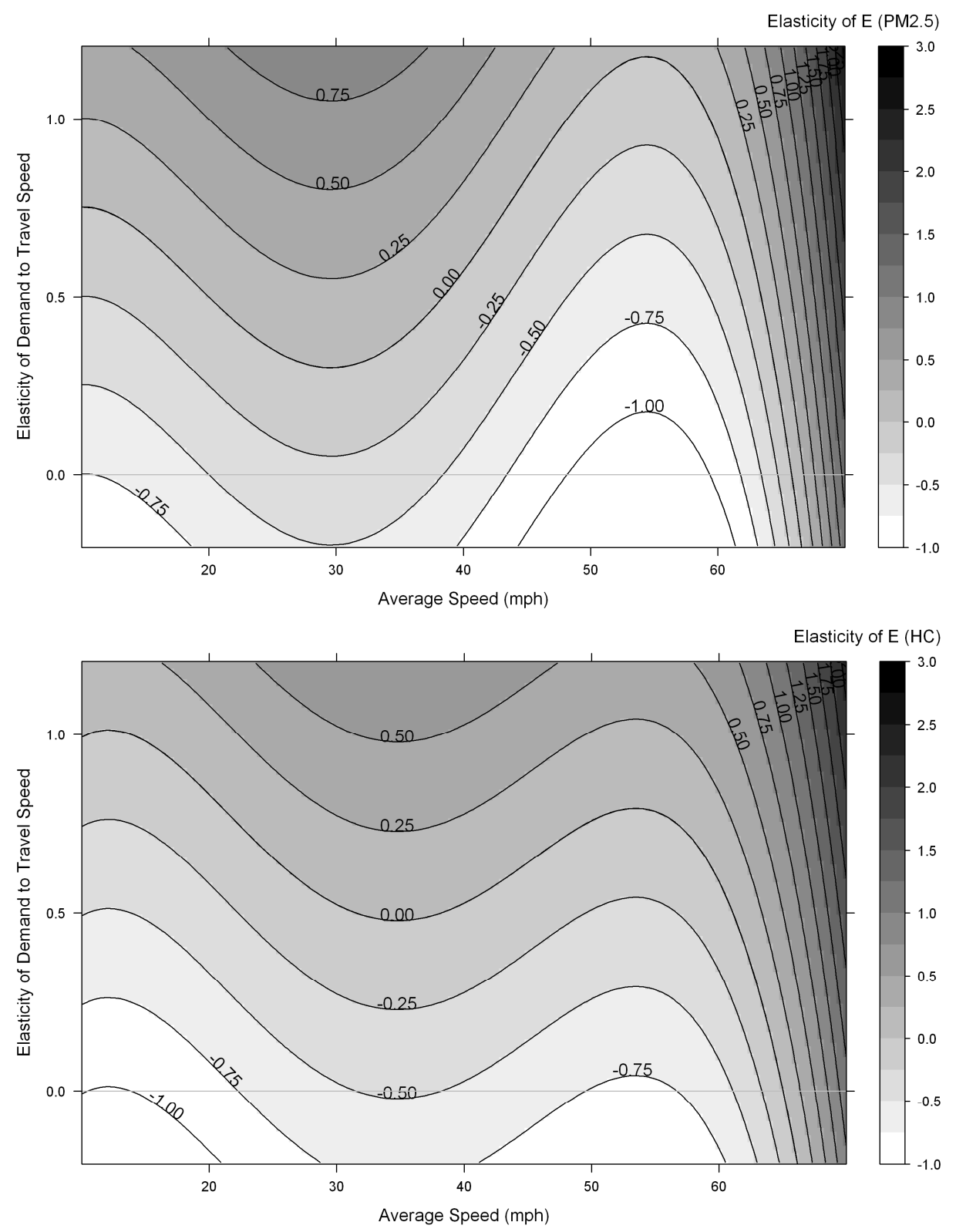

Figure 15. (Part II) Elasticity of Total Emissions to Speed, $\varepsilon_{E}^{\bar{v}}$, for $\mathrm{PM}_{2.5}$ and $\mathrm{HC}$, as

\section{Shadings and Contours on the $\eta_{q}^{\bar{v}}$ versus $\bar{v}$ Plane}

\subsection{Emissions Break-Even Demand Elasticity to Speed}

We continue the inspection of emissions break-even conditions by illustrating the graphical approach described in Section 4.7.2. Figure 16 shows total $\mathrm{CO}_{2} \mathrm{e}$ emissions contour shadings ( $E$ in $\mathrm{kg} / \mathrm{hr} /$ lane-mi) on the average travel speed ( $\bar{v}$ in mph) versus 
effective demand volume ( in pcphpl) plane. For illustrative purposes, assume that (no PCE adjustment). The two BPR-derived curves on the plot use freeway capacities of pcphpl (solid black line) and pcphpl (dashed black line) - a $10 \%$ increase.

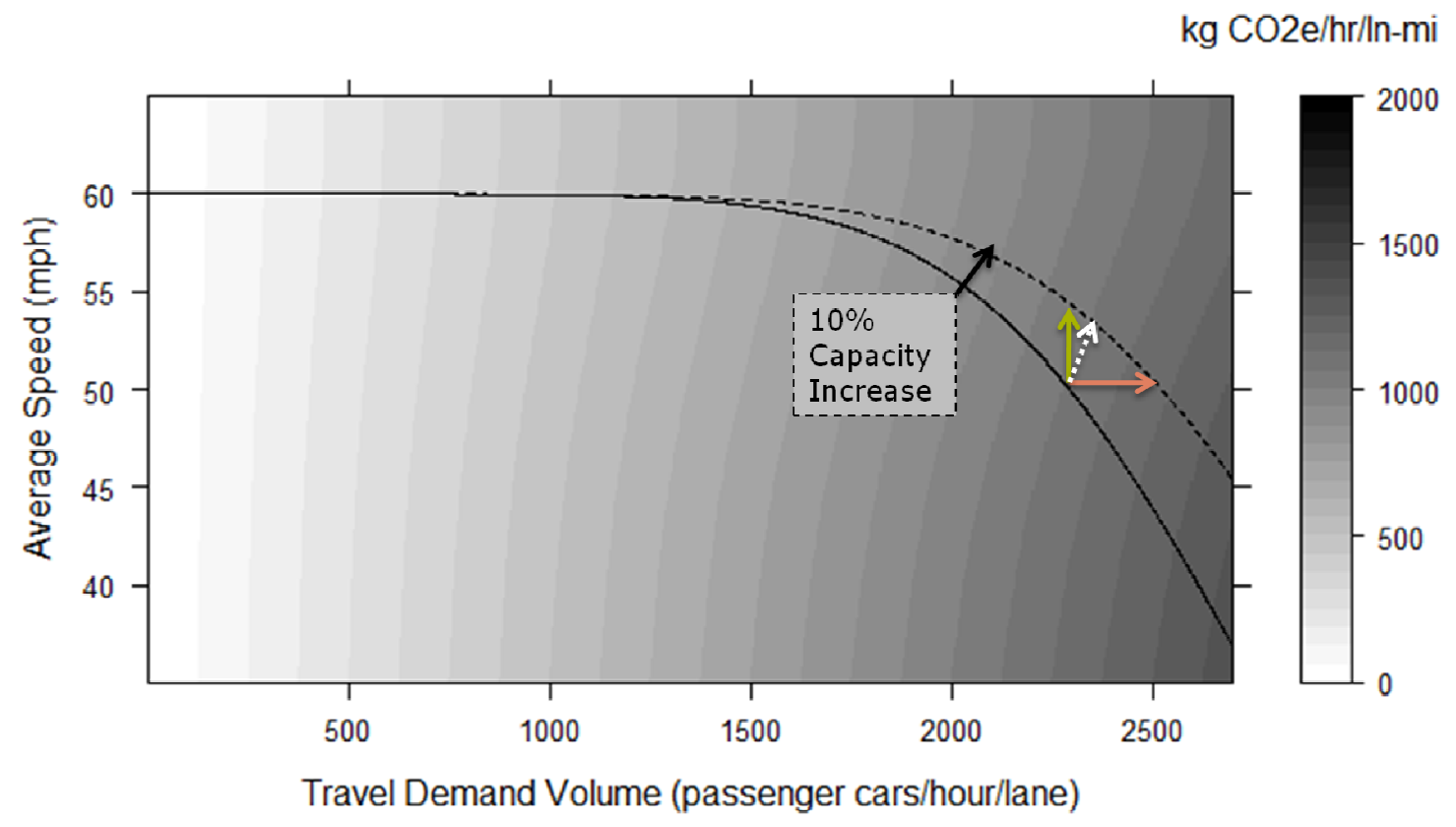

Figure 16. The Effect of a Capacity Increase on Total Emissions Depends on the Final Demand Volume; Shading is Total Freeway $\mathrm{CO}_{2}$ e Emissions Rate, , by the MOVES Model

As an illustration, consider an initially congested demand state of pcphpl during a peak period, with an initial emissions rate of $\mathrm{kg} / \mathrm{hr} / \mathrm{lane}$-mi. If the capacity were to increase by $10 \%$, travel speed would increase by $7 \%$ and the total emissions would decrease by $3 \%$ - at a fixed demand flow of pcphpl. This is the vertical green arrow on Figure 16. If, alternatively, the travel speed were fixed (i.e. the constant travel time budget scenario suggest by Metz (2008)), the new demand flow would be pcphpl, with a total emissions, , increase of $10 \%$. This is the 
horizontal orange arrow on Figure 16. The most likely long-term outcome is some induced demand and some travel time savings, ending up on the lower curve somewhere between these two extremes.

To estimate a break-even induced demand volume from an emissions perspective we follow an emissions contour line up from the lower (solid black line) traffic curve to the upper (dashed black line) curve, arriving at an emissions-equivalent induced demand that would cancel marginal speed benefits (the dashed white arrow on Figure 16). For the example here, the original emissions are found on the upper curve at a volume of $q=2,252 \mathrm{pcphpl}-$ which corresponds to a $2.4 \%$ increase in flow and a $5.4 \%$ increase in travel speed. Thus the emissions break-even elasticity of travel demand to average travel speed is $\gamma_{q}^{\bar{v}}=0.44$ (calculated as the mid-point/linear arc elasticity - see Equation 12). This is a moderate value in comparison to the literature, which generally ranges from 0.2 to 1 (see Section 2.5).

As a comparison among models, full-fleet freeway $\mathrm{CO}_{2}$ and $\mathrm{CO}_{2} \mathrm{e}$ break-even elasticities of travel demand volume to average travel speed, $\gamma_{q}^{\bar{v}}$, are shown in Figure 17 for the three macroscopic emissions models used above for emissions rate modeling: MOVES, Barth, and Boulter. The MOVES and Barth models, formulated as exponentiated polynomials, use Equation 38. The Boulter model is formulated as a nonexponentiated $5^{\text {th }}$-order polynomial and is simply differentiated with respect to speed using $\gamma_{q}^{\bar{v}}=-\varepsilon_{\bar{e}}^{\bar{v}}=-\frac{\bar{v}}{\bar{e}} \cdot \frac{\partial \bar{e}}{\partial \bar{v}}$. The emissions break-even elasticities $\gamma_{q}^{\bar{v}}$ can be interpreted as 'carbon-neutral' curves for demand elasticity. True elasticities above the curves $\left(\eta_{q}^{\bar{v}}>\right.$ $\left.\gamma_{q}^{\bar{v}}\right)$ are expected to increase total $\mathrm{CO}_{2}$ emissions from traffic speed increases and true 
elasticities below the curves $\left(\eta_{q}^{\bar{v}}<\gamma_{q}^{\bar{v}}\right)$ are expected to decrease total $\mathrm{CO}_{2}$ emissions from traffic speed increases. By Equation 39 the vertical distance between the break-even elasticity curve and the true elasticity is the elasticity of total emissions to travel speedthe farther the distance, the greater the emissions impact, positive or negative.

The results in Figure 17 are highly intuitive in light of the preceding analysis. Assuming a moderate demand elasticity of $\eta_{q}^{\bar{v}}=0.5$, only in heavily congested freeway conditions is it possible to reduce total emissions through traffic speed increases. For more elastic demand near $\eta_{q}^{\bar{v}}=1.0$, induced demand will always increase total emissions with a traffic speed increase. By the MOVES model, any elasticity above 0.4 would likely lead to increased total emissions for speed increases from an initial speed over $25 \mathrm{mph}$.

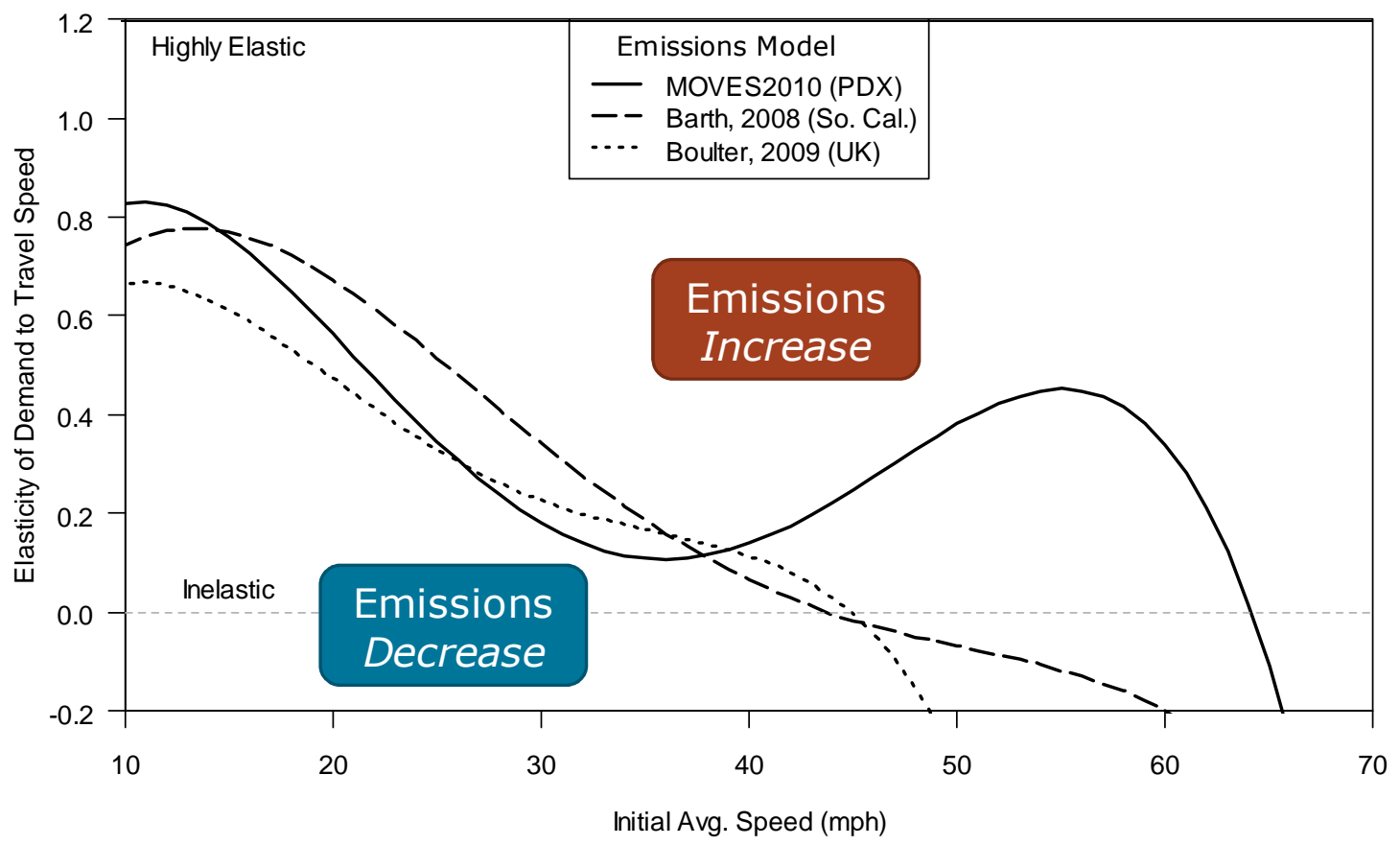

Figure 17. Calculated Emissions Break-Even Elasticities of Travel Demand to Travel Speed, $\gamma_{q}^{\bar{v}}$, for $\mathrm{CO}_{2}$ (Barth and Boulter) and $\mathrm{CO}_{2} \mathrm{e}$ (MOVES) 
At high speeds the MOVES model produces $\gamma_{q}^{\bar{v}}$ notably different from the other two models. Emissions rates $\bar{e}$ decrease with speed when approaching free-flow speed (around 60mph) in the MOVES model, but increase with speed when approaching freeflow speed by the other two models (see Figure 4). Break-even elasticities below zero $\left(\gamma_{q}^{\bar{v}}<0\right)$ indicate that emissions rates increase with higher speeds - even before induced demand. Hence, in the Barth and Boulter models increasing freeway speed over $45 \mathrm{mph}$ always increases emissions.

Figure 18 presents calculated $\gamma_{q}^{\bar{v}}$ using the MOVES-modeled emissions rates for freeways and arterials and the Boulter emissions model (which does not segment by facility type), calculated as for Figure 17, for the other four pollutants modeled. Note that the vertical scale in Figure 18 is different from Figure 17, to accommodate higher values of $\gamma_{q}^{\bar{v}}$. There is less agreement here among the models than in Figure 17, which is not surprising since not only do other pollutants generally have more modeling uncertainty than $\mathrm{CO}_{2}$, but the emissions controls for these pollutants are different between the U.S. and the U.K. The emissions models produce particularly different $\gamma_{q}^{\bar{v}}$ for speeds above 40 mph. 

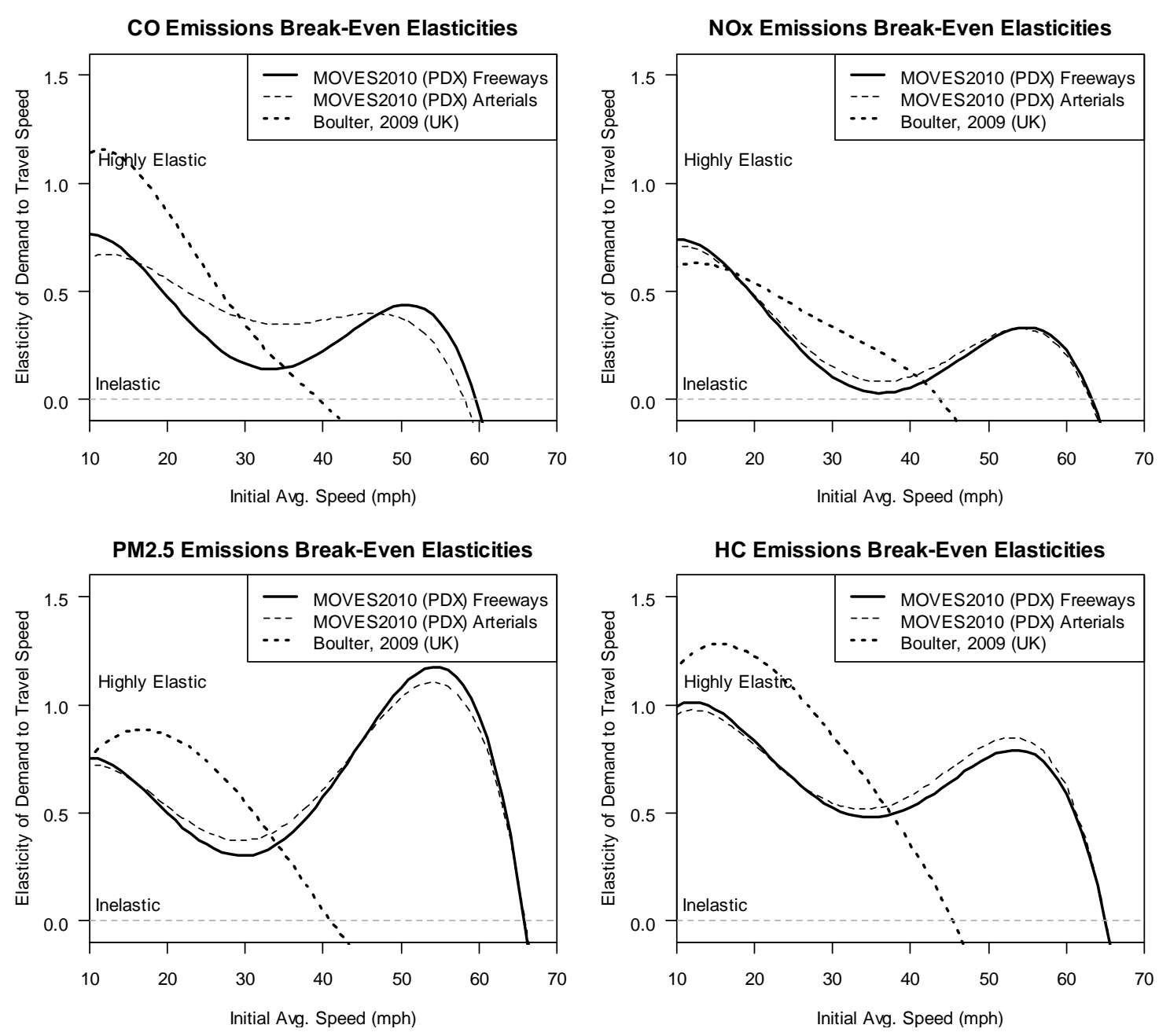

Figure 18. $\gamma_{q}^{\bar{v}}$ for Different Pollutants versus $\bar{v}$

$\mathrm{PM}_{2.5}$ and $\mathrm{HC}$ emissions are more sensitive to speed than the other pollutants and thus have generally larger $\gamma_{q}^{\bar{v}}$ in Figure 18. As in Figure 15, this means that $\mathrm{PM}_{2.5}$ and $\mathrm{HC}$ emissions are more likely to decrease with speed increases (or increase with speed decreases) than other pollutants. The $\gamma_{q}^{\bar{v}}$ for $\mathrm{PM}_{2.5}$ are particularly high around $50-60$ mph (near freeway free-flow speeds). Similarly, $\mathrm{CO}$ emissions on arterials are more likely to benefit from speed improvements than $\mathrm{CO}$ emissions on freeways - assuming 
the demand elasticities are the same - since the arterial break-even elasticity curve is higher than the freeway curve for $\mathrm{CO}$.

Although the break-even elasticities vary by emissions model, pollutant, and initial speed, almost all $\gamma_{q}^{\bar{v}}$ values here are within a reasonable long-term range of demand elasticity to travel speed, between 0 and 1 . Values of $\gamma_{q}^{\bar{v}}$ closer to zero are more feasibly reached on a short time scale - which is the case for most pollutants at moderate initial speeds. For lower initial speeds below $25 \mathrm{mph}$, the marginal emissions rate benefits of speed increases are greater, and so less likely (though still possible) to be offset by induced demand. Figure 17 and Figure 18 show that in most situations it is likely that traffic speed increases will increase emissions in the long-run by the induced demand effect, though the time required for induced demand to cancel marginal emissions rate benefits would be longer for heavier initial congestion.

\subsection{Total Emissions Elasticity to Travel Demand Volume}

Combining Equations 35 and 37, the full-fleet total emissions elasticity to volume changes is

$$
\varepsilon_{E}^{q}=1+\varepsilon_{\bar{e}}^{\bar{v}} \cdot \frac{-\alpha \beta \frac{\partial q^{\prime}}{\partial q}}{\left(\left(\frac{c}{q^{\prime}}\right)^{\beta}+\alpha\right) \sum_{\mathrm{j} \in \mathrm{J}}\left(P C E_{j} \cdot f_{j}\right)}
$$

Assuming that $\frac{\partial q^{\prime}}{\partial q}=\frac{q^{\prime}}{q}=\sum_{\mathrm{j} \in \mathrm{J}}\left(P C E_{j} \cdot f_{j}\right)-$ the PCE-adjusted flow grows proportionally with the volume flow - we can calculate $\varepsilon_{E}^{q}$ using the previous values for $\alpha, \beta$, c, and $t_{o}$, Equation 17 for $\varepsilon_{\bar{e}}^{\bar{v}}$, the BPR function (Equation 1), and the ESC fit parameters from Table 4. The calculated values for Elasticity of Total $\mathrm{CO}_{2} \mathrm{e}$ Emissions to Volume, $\varepsilon_{E}^{q}$, are 
shown in Figure 19 along with the Elasticity of Emissions Rate to Speed, $\varepsilon_{\bar{e}}^{\bar{v}}$, and the Elasticity of Speed to Volume, $\varepsilon_{\bar{v}}^{q}$. These three elasticities are the components of Equation 35, where $\varepsilon_{E}^{q}=1+\varepsilon_{\bar{e}}^{\bar{v}} \varepsilon_{\bar{v}}^{q}$.

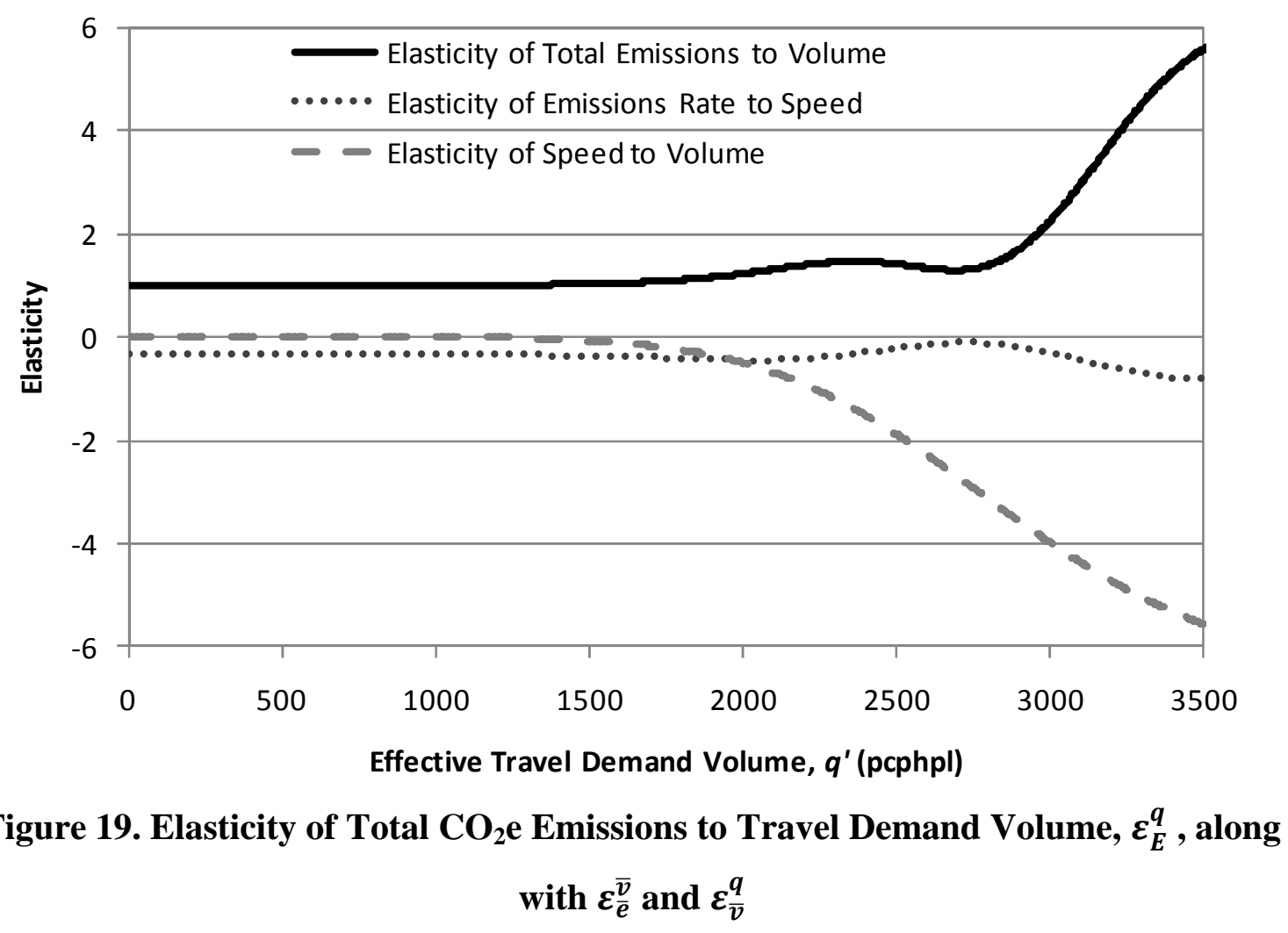

Figure 19 shows $q^{\prime}$ values up to 3,500 pcphpl - which corresponds to an average travel speed of about $12 \mathrm{mph}$. For volumes below about $q^{\prime}=2,000 \mathrm{pcphpl}$, the speed effects are minimal, as expected. Since $\varepsilon_{\bar{e}}^{\bar{v}}$ and $\varepsilon_{\bar{v}}^{q}$ are both negative, $\varepsilon_{E}^{q}$ increases at higher values of $q^{\prime}$ and is always at 1 or above. The increase in $\varepsilon_{E}^{q}$ is especially dramatic for $q^{\prime}$ above 3,000 pcphpl (where $\bar{v}$ drops below $26 \mathrm{mph}$ ), since $\varepsilon_{\bar{e}}^{\bar{v}}$ is large (close to -1) and $\varepsilon_{\bar{v}}^{q}$ decreases rapidly. As noted above, these curves depend on a fixed volume-speed relationship (the BPR function) and so do not reflect changing roadway conditions (particularly capacity changes). Still, Figure 19 shows that for a fixed capacity roadway 
increasing volumes is always expected to increase total emissions. The effect is larger at demand volumes well above capacity, where emissions rates increase rapidly with

demand volume as well (since $\varepsilon_{\bar{e}}^{q}=\varepsilon_{\bar{e}}^{\bar{v}} \cdot \varepsilon_{\bar{v}}^{q}$ from Equation 33). Finally, since $\varepsilon_{\bar{e}}^{q}=\varepsilon_{E}^{q}-$ 1 (see Equation 35 ), we see that $\varepsilon_{\bar{e}}^{q}$ will be close to 0 for volumes up to about 2,800 pcphpl.

In this chapter we have shown that:

1. Emissions rates are "stagnant" at a wide range of moderate speeds,

2. Emissions rates are more sensitive to speed on arterials than on freeways and for local pollutants than for greenhouse gases,

3. Varying demand volume can outweigh changing efficiency for total emissions effects of travel speed changes, and

4. Total emissions reductions from travel speed increases are only likely for low demand elasticity and low travel speeds.

In the next chapter we look at how the total emissions picture changes when we incorporate advanced-drivetrain vehicles. 


\section{THE IMPACTS OF ADVANCED VEHICLE TECHNOLOGIES}

The results in Chapter 5 are for conventional Internal Combustion Engine (ICE) vehicles only - the vast majority of the existing on-road fleet (U.S. Environmental Protection Agency, 2009b). In this chapter we look at the effects of advanced drivetrain and electric vehicles in the fleet. Let vehicle class $j=c$ stand for all conventional Internal Combustion Engine (ICE) vehicles, vehicle class $j=e$ stand for Electric Vehicles (EV), and vehicle class $j=a$ stand for Advanced Drivetrain (AD) vehicles. We assume this is the complete set of vehicles, $J=\{c, e, a\}$.

The AD vehicle class contains vehicles (such as gas-electric hybrids) with regenerative breaking and other powertrain efficiencies which render them less sensitive or insensitive to low-speed inefficiencies. If we are interested in local pollutants, then

$e_{e}=0$ since EV's have zero on-road air pollution emissions. By extension, $\frac{\partial e_{e}}{\partial v_{e}}=0$. Let us assume for this analysis that $\mathrm{AD}$ vehicles in class $j=a$ have emissions rates which are not zero $\left(e_{a} \neq 0\right)$, but which are insensitive to congestion level and average speed: $\frac{\partial e_{a}}{\partial v_{a}}=0$

\subsection{Emissions Rate Sensitivity to Speed and Advanced Vehicles}

The average emissions rate from a mixed vehicle fleet including EV and AD vehicles is $\bar{e}=f_{c} e_{c}+f_{a} e_{a}$ (from Equation 4). Since $\frac{\partial e_{a}}{\partial v_{a}}=\frac{\partial e_{e}}{\partial v_{e}}=0$, we know that $\varepsilon_{e_{a}}^{v_{a}}=\varepsilon_{e_{e}}^{v_{e}}=0$. Then from Equation 28 (which assumes proportional speed changes among the vehicle classes)

$$
\frac{\partial \bar{e}}{\partial \bar{v}}=\frac{1}{\bar{v}}\left(e_{c} \cdot f_{c} \cdot \varepsilon_{e_{c}}^{v_{c}}\right)=f_{c} \frac{v_{c}}{\bar{v}} \frac{\partial e_{c}}{\partial v_{c}}
$$


Compared to the ICE-only gradient $\frac{\partial e_{c}}{\partial v_{c}}$, if we assume that $v_{c} \approx \bar{v}$, then $\frac{\partial \bar{e}}{\partial \bar{v}}$ decreases proportionally with $f_{c}$, the fraction of ICE vehicles in the fleet. With a higher fraction of EV and AD vehicles, the gradients in Figure 4 would be proportionally closer to zero.

For emissions rate elasticity to average speed, from Equations 29 and 43,

$$
\varepsilon \overline{\bar{v}}=f_{c} \frac{v_{c}}{\bar{e}} \frac{\partial e_{c}}{\partial v_{c}}=\frac{f_{c} e_{c}}{f_{c} e_{c}+f_{a} e_{a}} \cdot \varepsilon_{e_{c}}^{v_{c}}=\frac{E_{c}}{E} \varepsilon_{e_{c}}^{v_{c}} .
$$

Again comparing with the ICE-only elasticity $\varepsilon_{e_{c}}^{v_{c}}$, here we see that $\varepsilon_{\bar{e}}^{\bar{v}}$ decreases with the fraction of ICE emissions out of total emissions. Hence, $\varepsilon \overline{\bar{v}}$ decreases with increasing AD vehicle emissions, $E_{a}$, since the $\mathrm{AD}$ vehicle emissions are insensitive to speed changes. Furthermore, if the presence of EV's does not affect the relative proportions of AD and ICE vehicles, $f_{a} / f_{c}$, then the presence of EV's will not impact $\varepsilon_{\bar{e}}^{\bar{v}}$.

\subsection{Total Emissions Sensitivity to Speed and Advanced Vehicles}

Total emissions, $E$, from a mixed vehicle fleet including EV and AD vehicles are

$$
E=q\left[f_{c} e_{c}+f_{a} e_{a}\right]
$$

Again assuming that the presence of EV's does not affect the relative proportions of AD and ICE vehicles, $f_{a} / f_{c}$, then the impact on $E$ of EV's is simply a proportional reduction in $E$ equal to the $\mathrm{EV}$ proportion of the fleet, $f_{e}$. For total emissions elasticity to speed, from Equation 19 we know that $\varepsilon_{E_{e}}^{v_{e}}=0$ and $\varepsilon_{E_{a}}^{v_{a}}=\eta_{q_{a}}^{v_{a}}$, since $\varepsilon_{e_{a}}^{v_{a}}=\varepsilon_{e_{e}}^{v_{e}}=0$. Then, from Equation 26, for a mixed ICE/EV/AD fleet

$$
\frac{\partial E}{\partial \bar{v}}=\frac{q}{\bar{v}}\left(f_{c} \cdot e_{c} \cdot \varepsilon_{E_{c}}^{v_{c}}+f_{a} \cdot e_{a} \cdot \eta_{q_{a}}^{v_{a}}\right) .
$$


For a fleet of only ICE and EV vehicles $\left(f_{a}=0\right)$, the rate of change of total emissions with traffic speed shrinks proportionally with decreasing fraction of ICE's in the fleet, $f_{\mathrm{c}}$. For an increasing fraction of $\mathrm{AD}$ vehicles, $f_{a}$, if the $\mathrm{AD}$ vehicles are replacing $\mathrm{EV}$ 's then $\frac{\partial E}{\partial \bar{v}}$ is expected to increase with $f_{a}$ (because of variable demand). If the AD vehicles are replacing ICE vehicles, then the change in $\frac{\partial E}{\partial \bar{v}}$ depends on the relative emissions rates and elasticities, $e_{a}, e_{c}, \eta_{q_{a}}^{v_{a}}$, and $\varepsilon_{e_{c}}^{v_{c}}$.

In terms of emissions elasticity to speed, using the assumption that $\eta_{q_{a}}^{v_{a}}=\eta_{q_{c}}^{v_{c}}$, then from Equation 27

$$
\varepsilon_{E}^{\bar{v}}=\frac{f_{c} \cdot e_{c} \cdot \varepsilon_{E_{c}}^{v_{c}}+f_{a} \cdot e_{a} \cdot \eta_{q_{a}}^{v_{a}}}{f_{c} \cdot e_{c}+f_{a} \cdot e_{a}}=\varepsilon_{E_{c}}^{v_{c}}-\frac{E_{a}}{E} \varepsilon_{e_{c}}^{v_{c}}
$$

For a fleet of only EV and ICE vehicles $\left(f_{a}=0\right)$, the elasticity is unaffected by the presence of EV's, $\varepsilon_{E}^{\bar{v}}=\varepsilon_{E_{c}}^{v_{c}}$. With an increasingly high fraction of AD vehicles, $f_{a}$, the elasticity increases (becomes more positive), since $\varepsilon_{e_{c}}^{v_{c}}$ is expected to be negative through most of the range of feasible speeds - see Figure 13. This makes sense, as the AD vehicles do not see the efficiency improvements of ICE vehicles with increasing speed, but still are subject to increased emissions through induced demand.

\subsection{Break-Even Demand Elasticity and Advanced Vehicles}

Equation 40 implies emissions break-even conditions when

$$
f_{a} \cdot e_{a} \cdot \eta_{q_{a}}^{v_{a}}=-f_{c} \cdot e_{c} \cdot \varepsilon_{E_{c}}^{v_{c}}
$$

If we again assume that $\eta_{q_{a}}^{v_{a}}=\eta_{q_{c}}^{v_{c}}$, then emissions break-even conditions exist when

$$
\eta_{q_{a}}^{v_{a}}=\eta_{q_{c}}^{v_{c}}=\frac{f_{c} \cdot e_{c}}{f_{a} \cdot e_{a}+f_{c} \cdot e_{c}} \cdot \gamma_{q_{c}}^{v_{c}}=\frac{E_{c}}{E} \gamma_{q_{c}}^{v_{c}}
$$


Thus the break-even demand elasticity with AD vehicles is proportionally smaller than for ICE vehicles alone, in proportion to the fractional ICE emissions out of total emissions. As with $\varepsilon_{\bar{e}}^{\bar{v}}$ and $\varepsilon_{E}^{\bar{v}}$, if the presence of EV's does not affect the relative

proportions of $\mathrm{AD}$ and ICE vehicles, $f_{a} / f_{c}$, then the EV's will not impact break-even demand elasticity.

\subsection{Summary of Advanced Vehicle Impacts}

In summary, emissions rates from fleets with advanced vehicles are less sensitive to speed changes than all-ICE fleets. But because AD vehicles decrease emissions rate sensitivity to speed while still experiencing variable demand, they increase (make more positive) total emissions elasticity to speed. Similarly, the break-even demand elasticity of a fleet with AD vehicles is smaller than that of a fully ICE fleet. Although EV's decrease total emissions and emissions gradients, they do not affect emissions elasticity to speed for local pollutants (since the induced demand emissions are zero).

These results show that emissions from fleets with more advanced vehicles are less sensitive to congestion. As vehicle emissions rates become less sensitive to speed, the break-even demand elasticity gets smaller. Thus, the potential for emissions benefits from congestion mitigation will decrease with more advanced vehicles. In the next chapter we extend the analysis to consider heavy-duty and light-duty portions of the fleet separately. 


\section{THE IMPACTS OF HEAVY VEHICLES}

In this chapter we compare the differing impacts of two ICE vehicle classes: lightduty $(j=l)$ and heavy-duty $(j=h)$. Thus, $J=\{l, h\}$, where $l$ and $h$ are differentiated as described in Section 4.3.2. These two vehicle classes have emissions rates $e_{l}$ and $e_{h}$ (calculated from Equation 3), and volume flows $q_{l}$ and $q_{h}$.

\subsection{Emissions Rates and HD/LD Vehicle Classes}

The MOVES-fitted parameters for emissions rates (by Equation 5 using $n=4$ ) of LD and HD portions of the vehicle fleet are shown separately in Table 7 and Table 8 for the PM peak periods on freeways in April, 2010. Figure 20 illustrates the emissions rate relationships between $\mathrm{LD}$ and $\mathrm{HD}$ vehicle classes and average speed for all five pollutants as the ratio of HD to LD emissions rates, ${ }^{e_{h}} / e_{l}$, assuming $v_{l}=v_{h}=\bar{v}$.

Table 7. MOVES Emissions-Speed Curve Fit Parameters for $\boldsymbol{e}_{l}$ on Freeways

\begin{tabular}{|c|r|r|r|r|r|}
\hline Freeways & \multicolumn{1}{|c|}{$\mathbf{C O}_{\mathbf{2}} \mathbf{e}$} & \multicolumn{1}{c|}{$\mathbf{C O}$} & \multicolumn{1}{c|}{$\mathbf{P M}_{\mathbf{2 . 5}}$} & \multicolumn{1}{c|}{$\mathbf{N O}_{\mathbf{x}}$} & \multicolumn{1}{c|}{$\mathbf{H C}$} \\
\hline$a_{0, l}$ & 7.987 & 2.788 & -2.856 & 0.3239 & -0.2644 \\
\hline$a_{1, l}$ & -0.1856 & -0.1760 & -0.2000 & -0.1152 & -0.1878 \\
\hline$a_{2, l}$ & 0.006352 & 0.006535 & 0.007365 & 0.004155 & 0.006173 \\
\hline$a_{3, l}$ & $-9.550 \mathrm{E}-05$ & $-1.077 \mathrm{E}-04$ & $-1.157 \mathrm{E}-04$ & $-6.270 \mathrm{E}-05$ & $-9.570 \mathrm{E}-05$ \\
\hline$a_{4, l}$ & $5.210 \mathrm{E}-07$ & $6.460 \mathrm{E}-07$ & $6.560 \mathrm{E}-07$ & $3.440 \mathrm{E}-07$ & $5.510 \mathrm{E}-07$ \\
\hline
\end{tabular}

Table 8. MOVES Emissions-Speed Curve Fit Parameters for $\boldsymbol{e}_{h}$ on Freeways

\begin{tabular}{|c|r|r|r|r|r|}
\hline Freeways & \multicolumn{1}{c|}{$\mathbf{C O}_{2} \mathbf{e}$} & \multicolumn{1}{c|}{$\mathbf{C O}$} & \multicolumn{1}{c|}{$\mathbf{P M}_{2.5}$} & \multicolumn{1}{c|}{$\mathbf{N O}_{\mathbf{x}}$} & \multicolumn{1}{c|}{$\mathbf{H C}$} \\
\hline$a_{0, h}$ & 9.254 & 3.541 & 1.005 & 4.124 & 2.059 \\
\hline$a_{1, h}$ & -0.1748 & -0.1900 & -0.1740 & -0.1839 & -0.2206 \\
\hline$a_{2, h}$ & 0.006307 & 0.006843 & 0.006599 & 0.006461 & 0.006967 \\
\hline$a_{3, h}$ & $-1.007 \mathrm{E}-04$ & $-1.097 \mathrm{E}-04$ & $-1.141 \mathrm{E}-04$ & $-1.003 \mathrm{E}-04$ & $-1.018 \mathrm{E}-04$ \\
\hline$a_{4, h}$ & $5.740 \mathrm{E}-07$ & $6.201 \mathrm{E}-07$ & $6.870 \mathrm{E}-07$ & $5.599 \mathrm{E}-07$ & $5.380 \mathrm{E}-07$ \\
\hline
\end{tabular}




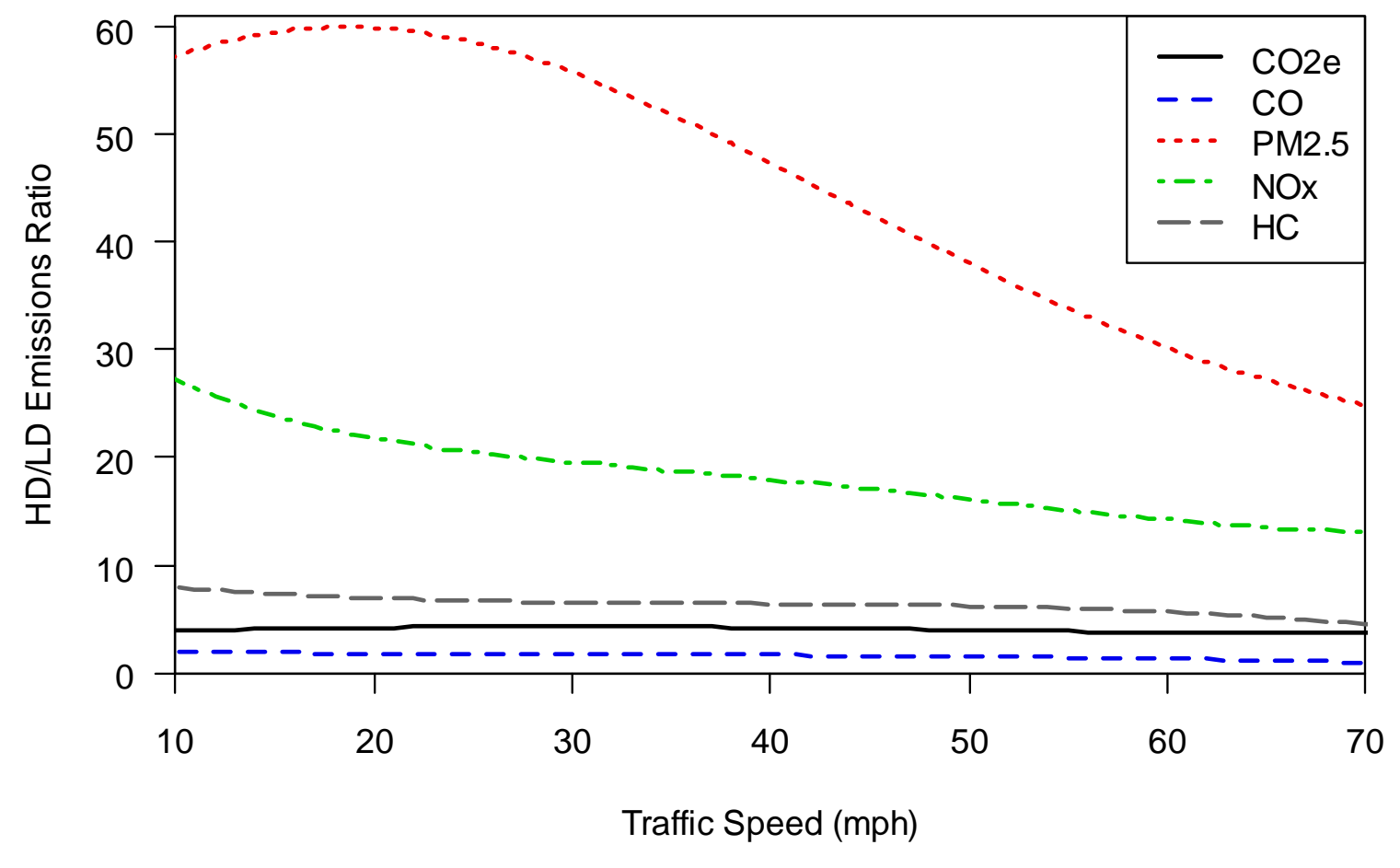

Figure 20. ${ }^{e_{h}} / e_{l}$ versus $\bar{v}$

The major $\mathrm{HD}$ vehicle pollutants are $\mathrm{PM}_{2.5}$ and $\mathrm{NO}_{\mathrm{x}}$, which reach factors of more than 60 and 25 times the LD vehicle emissions rates, respectively. By the generally downward sloping trends of the curves Figure 20 we see that HD vehicle low-speed inefficiencies are proportionally greater than LD vehicles' low-speed inefficiencies. In other words, HD vehicles' emissions rates increase faster in congestion. $\mathrm{CO}$ emissions rates are similar (a ratio of 1 to 2 ), $\mathrm{CO}_{2} \mathrm{e}$ emissions rates are about 4 times greater, and HC emissions rates are 4-8 times greater for HD vehicles than LD vehicles (per vehiclemile).

Some of the differences in Figure 20 relate to greater fuel consumption required to move heavier vehicles, as evidenced by 4 times higher $\mathrm{CO}_{2} \mathrm{e}$ emissions rates (which are closely tied to fuel consumption). For the extreme differences in $\mathrm{PM}_{2.5}$ and $\mathrm{NO}_{\mathrm{x}}$ the dominance of diesel fuel in the HD fleet and gasoline in the LD fleet is also an important 
factor. As an illustration, Figure 21 shows the emissions rate ratios for diesel versus gasoline-powered passenger cars (PC) and passenger trucks (PT). For this figure, we first define vehicle classes for gasoline PC $(j=\mathrm{pcg})$, diesel PC $(j=\mathrm{pcd})$, gasoline PT $(j=\mathrm{ptg})$, and diesel $\mathrm{PT}(j=\mathrm{ptd})$. We then compute $e_{j}$ using Equation 3 with the appropriate source types from Table 3. Finally, Figure 21 plots $e_{\mathrm{ptd}} / e_{\mathrm{ptg}}$ versus $\bar{v}$ on the left and $e_{\mathrm{pcd}} / e_{\mathrm{pcg}}$ versus $\bar{v}$ on the right (assuming $\bar{v}=v_{\mathrm{pcg}}=v_{\mathrm{pcd}}=v_{\mathrm{ptg}}=v_{\mathrm{ptd}}$ ). Note the different scales on the vertical axes in Figure 21, reflecting the fact that the diesel/gasoline differences are more pronounced for PT than PC. In both cases $\mathrm{PM}_{2.5}$ and $\mathrm{NO}_{\mathrm{x}}$ have the highest emissions rate ratios, though the ratios are many times greater for PT than PC. $\mathrm{CO}_{2} \mathrm{e}$ emissions rates are similar between the two, while $\mathrm{CO}$ emissions rates are lower for diesel vehicles. As with the HD/LD ratios, ${ }^{e_{h}} / e_{l}$, the difference is magnified at lower average speeds - showing that diesel LD vehicle emissions are more affected by congestion than gasoline LD vehicle emissions.
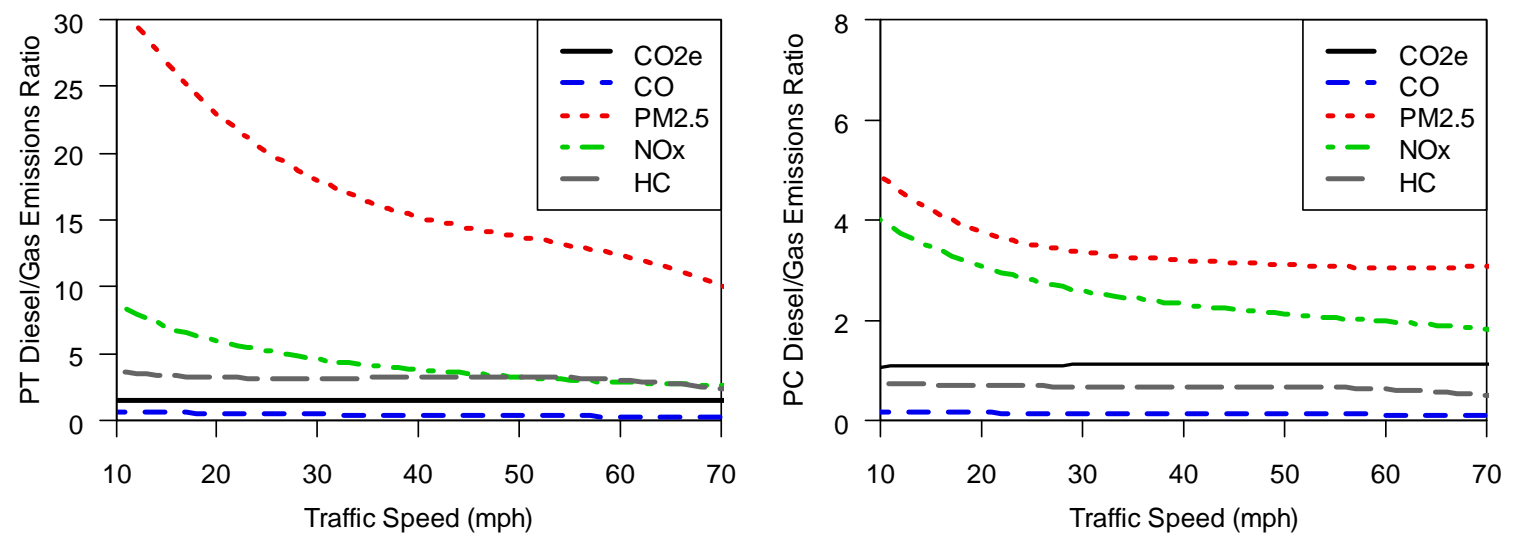

Figure 21. Diesel/Gasoline Vehicle Emissions Rate Ratios for Passenger Trucks (left) and Passenger Cars (right) 
Since light-duty and heavy-duty vehicles have distinct emissions characteristics, their combination in the total fleet affects the fleet-wide ESC and ESC gradients. Figure 22 shows the sensitivity to fraction HD vehicles, $f_{h}$, of fleet ESC and ESC gradients for all five pollutants modeled, with $\bar{e}$ computed from Equation 4 using $f_{h}$ from 0.0 to 0.5 . As in Figure 3, we include LOS indicators for freeways. No adjustment is made for PCE. As expected, higher $f_{h}$ increases the fleet emissions rates, $\bar{e}$ (seen in the left panels). The emissions rate increases are proportionally larger for pollutants with higher $e_{h} / e_{l}$ ratios in Figure 20. Fleet emissions rate sensitivity to speed also increases with $f_{h}-$ evidenced by the larger absolute values of the gradients in the right-side panels of Figure 22). This is expected from the downward sloping curves in Figure 20. For $\mathrm{PM}_{2.5}$ and $\mathrm{NO}_{\mathrm{x}}$, which are dominated by HD vehicle emissions, the gradient changes most dramatically with the initial introduction of HD vehicles (compare the gradients at $f_{h}=0 \%$ and $f_{h}=10 \%$ for these pollutants). Interestingly, the optimal speed also increases with $f_{h}$ - shown by the gradients crossing the horizontal (speed) axis at higher values with higher percentage HD. These plots show that traffic streams with more HD vehicles potentially have greater efficiency benefits from increasing average travel speeds. Also, because of their different emissions-speed relationships, LD and HD vehicles could be targeted separately for congestion mitigation with air quality objectives. 

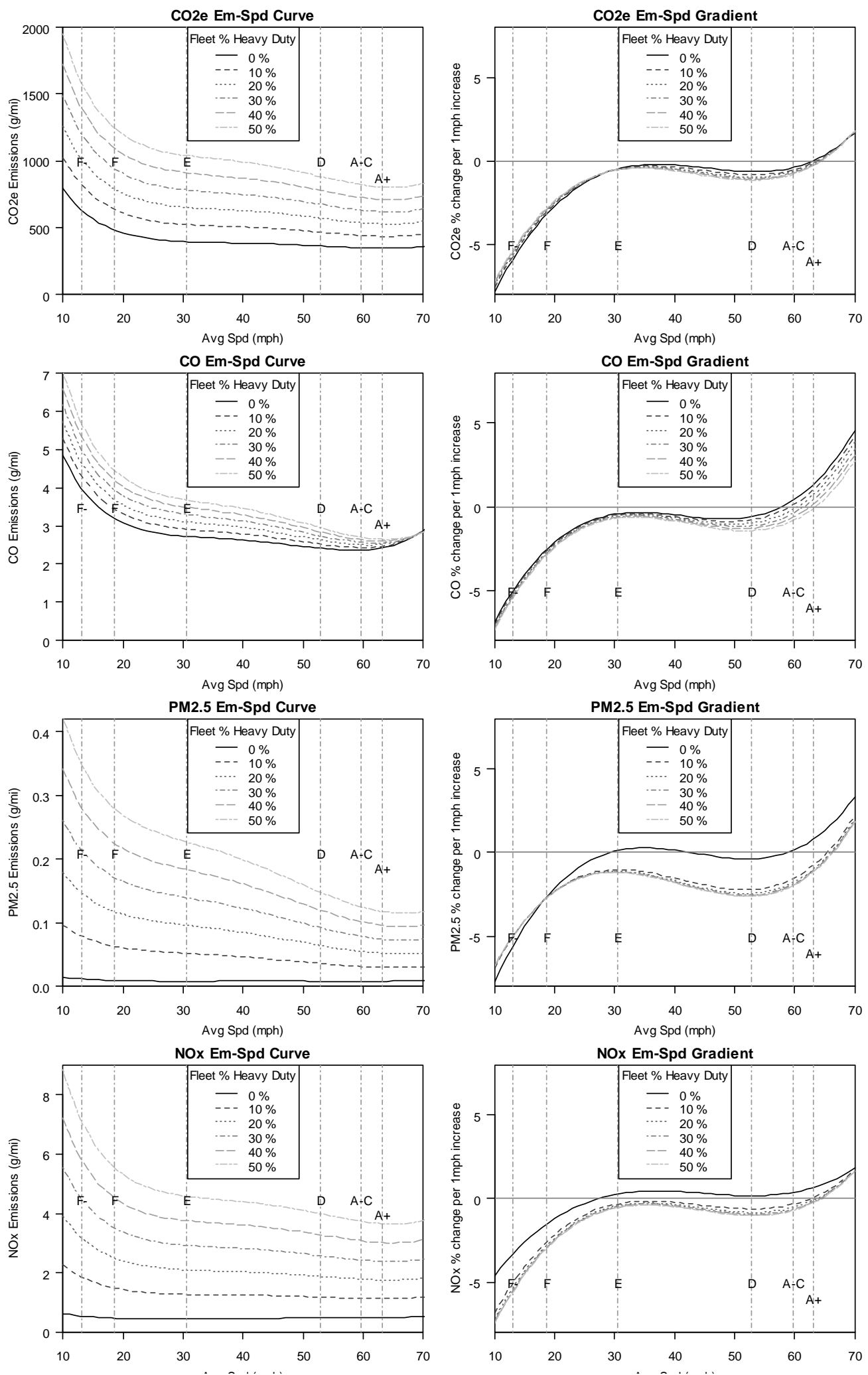

Figure 22 (Part I). Fleet Emissions Rate Sensitivity to $f_{h}$, with freeway LOS 

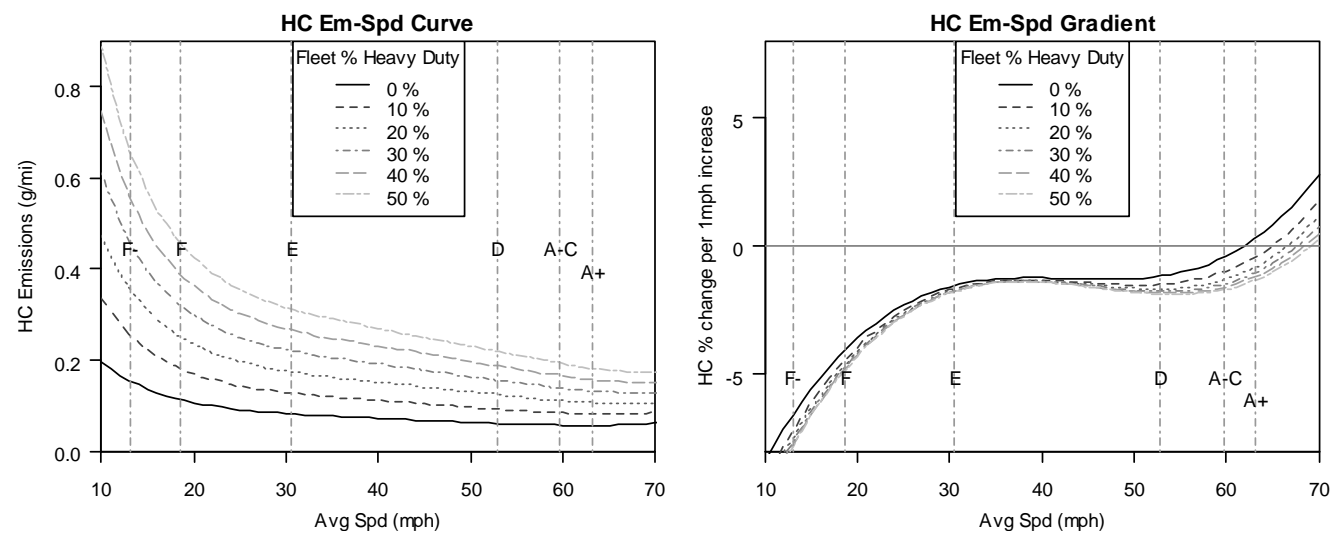

Figure 22 (Part II). Fleet Emissions Rate Sensitivity to $\boldsymbol{f}_{h}$, with freeway LOS

\subsection{Total Emissions and HD/LD Vehicle Classes}

We next look at how $f_{h}$ impacts total emissions, $E$. The total vehicle fleet emissions, from Equation 10, are

$$
E=q\left[\left(1-f_{h}\right) e_{l}+f_{h} e_{h}\right]
$$

Assuming $P C E_{l}=1$, then from Equation 2 the effective volume of vehicle travel (in PCE) is

$$
q^{\prime}=q\left(1+f_{h}\left(P C E_{h}-1\right)\right)
$$

A fleet of composed entirely of LD vehicles with the same effective flow rate $q^{\prime}$ would have total emissions of $E_{\text {all_LD }}=e_{l} \cdot q^{\prime}$. Using $P C E_{h}$, we can compare the two as

$$
E=q^{\prime}\left[\frac{\left(1-f_{h}\right) e_{l}+f_{h} e_{h}}{1+f_{h}\left(P C E_{h}-1\right)}\right]=E_{a l l-L D}\left[\frac{1+f_{h}\left(\frac{e_{h}}{e_{l}}-1\right)}{1+f_{h}\left(P C E_{h}-1\right)}\right]
$$

Using Equation 52 we can look at the impacts of heavy vehicles on total emissions, considering PCE, by looking at the ratio $E / E_{\text {all-LD }}$. For a given effective flow $q^{\prime}$ (adjusted for PCE), the total emissions from a mixed HD/LD fleet, $E$, as compared to the total emissions from an all-LD fleet, $E_{a l l-L D}$, are shown in Figure 23 as $E / E_{a l l-L D}$. 
These curves assume $P C E_{h}=1.5$ (for level terrain from the HCM (Transportation Research Board, 2000)), $f_{h}=0.1$ (10\% HD in the mixed fleet), and $v_{l}=v_{h}=\bar{v}$.

The results in Figure 23 are largely the same as those in Figure 20, but adjusted for $f_{h}$ and $P C E_{h}$. For $f_{h}=0.1$ and $P C E_{h}=1.5$, the effect of the $P C E_{h}$ adjustment is to reduce the impact of the presence of HD vehicles by $5 \%$ (see the denominator of Equation 52). In other words, since HD vehicles occupy more capacity than LD vehicles, the impact of HD vehicles' higher emissions rates $\left(\frac{e_{h}}{e_{l}}>1\right)$ are mitigated by $P C E_{h}>1$. As above for emissions rates, the presence of HD vehicles greatly increases total $\mathrm{PM}_{2.5}$ and $\mathrm{NO}_{\mathrm{x}}$ emissions - with a larger impact at slower speeds.

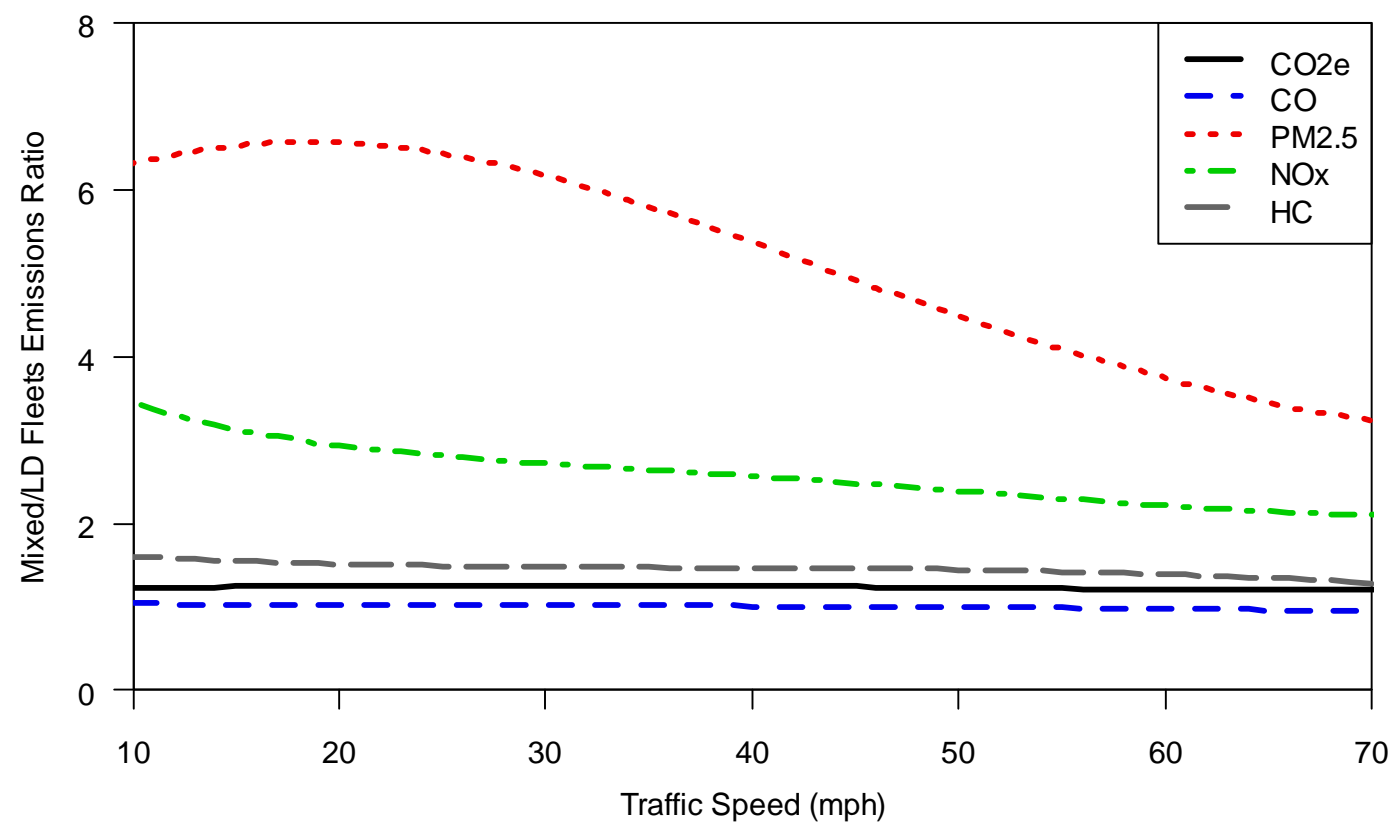

Figure 23. Comparison of Total Emissions from Mixed and LD-only Fleets, adjusted for PCE, as $E / E_{a l l-L D}$ versus $\bar{v}$ 


\subsection{Total Emissions Elasticity Considering Variable Demand and HD/LD Vehicle}

\section{Classes}

Figure 24 shows the class-specific freeway emissions break-even elasticities, $\gamma_{q_{j}}^{v_{j}}$, for $\mathrm{CO}_{2} \mathrm{e}$ by Equation 38 with the MOVES model. Similarly for the other pollutants we get Figure 25. Note the larger vertical scale in Figure 25 to accommodate the wider range of $\gamma_{q_{h}}^{v_{h}}$.

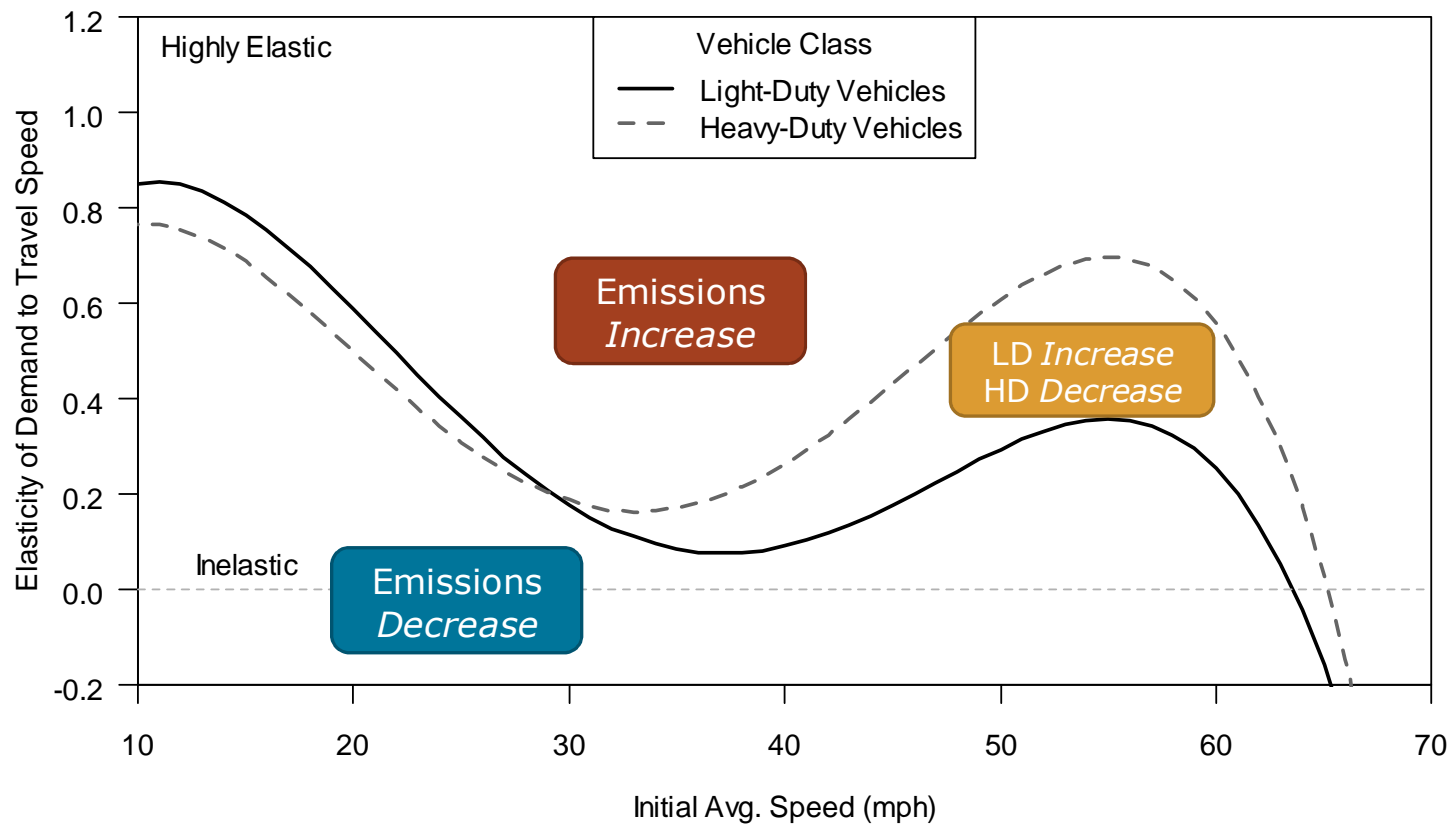

Figure 24. Vehicle Class-Specific Emissions Break-Even Elasticities for $\mathrm{CO}_{2}, \gamma_{q_{l}}^{v_{l}}$ and $\gamma_{q_{h}}^{v_{h}}$ versus $\bar{v}$

Figure 24 and Figure 25 show that in most cases the break-even elasticity for HD vehicles is higher than for LD vehicles, $\gamma_{q_{h}}^{v_{h}}>\gamma_{q_{l}}^{v_{l}}$. This occurs because HD vehicles are proportionally more inefficient at lower speeds (as illustrated in Figure 20). Because of this difference there is a range of true demand elasticities, $\eta_{q_{j}}^{v_{j}}$, between the curves for which we would expect total LD emissions, $E_{l}$, to increase but total HD emissions, $E_{h}$, to 
decrease with increasing speeds. So although the HD vehicle emissions rates are much higher for some pollutants, $e_{h}>e_{l}$, the potential for total emissions reductions through congestion mitigation can be higher, too - depending on the true demand elasticity for each vehicle class.
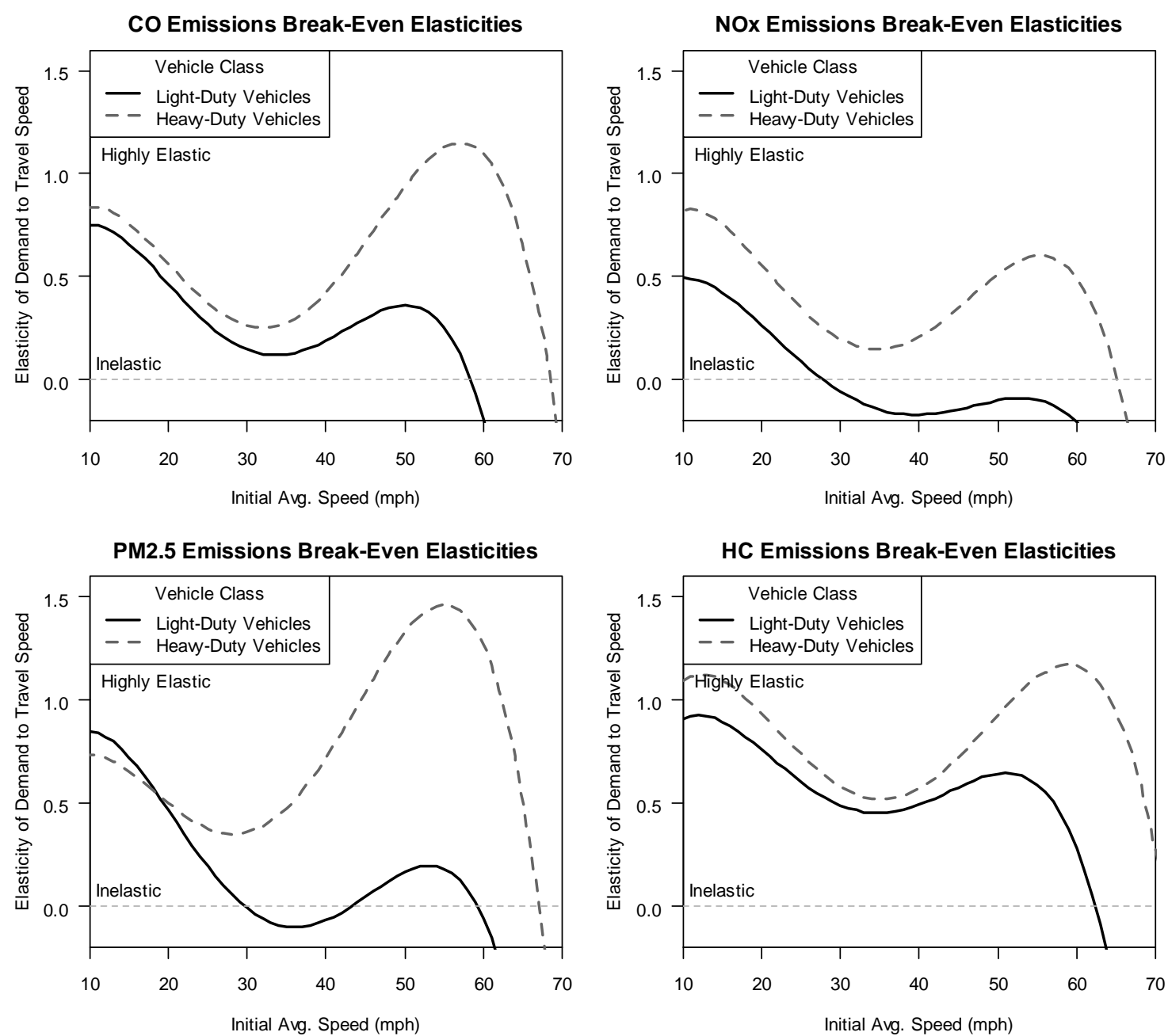

Figure 25. Vehicle Class-Specific Emissions Break-Even Elasticities for Other Pollutants, $\gamma_{q_{l}}^{v_{l}}$ and $\gamma_{q_{h}}^{v_{h}}$ versus $\bar{v}$

In addition to the break-even conditions for each vehicle class shown in Figure 24 and Figure 25, Equation 40 implies net even total emissions when 


$$
\left(f_{h}-1\right) e_{l} \varepsilon_{E_{l}}^{v_{l}}=f_{h} e_{h} \varepsilon_{E_{h}}^{v_{h}} \Rightarrow \frac{E_{l}}{E_{h}}=\frac{-\varepsilon_{E_{h}}^{v_{h}}}{\varepsilon_{E_{l}}^{v_{l}}}
$$

Thus, depending on each vehicle class's contribution to total emissions, a net emissions increase with speed from one class can be offset by a net emissions decrease with speed from the other.

Figure 24 and Figure 25 show the emissions reduction potentials of each vehicle class, but cumulative emissions changes depend on each vehicle class's share of the total emissions, as evidenced by Equation 27. HD vehicles' emissions rates can be many times larger than LD vehicles' emissions rates, but their portion of the total number of vehicles is typically smaller. Figure 26 shows the fraction of total fleet emissions that are from HD vehicles, $\frac{E_{h}}{E}$, assuming $f_{H}=0.1$ and $v_{l}=v_{h}=\bar{v}$. As could be expected, HD vehicle emissions dominate total $\mathrm{PM}_{2.5}$ and $\mathrm{NO}_{\mathrm{x}}$ emissions while $\mathrm{LD}$ vehicle emissions dominate total $\mathrm{CO}$ and $\mathrm{CO}_{2}$ emissions. All pollutants trend downward - toward a greater portion of total emissions from LD vehicles - since HD vehicles are comparatively more inefficient at low speeds 


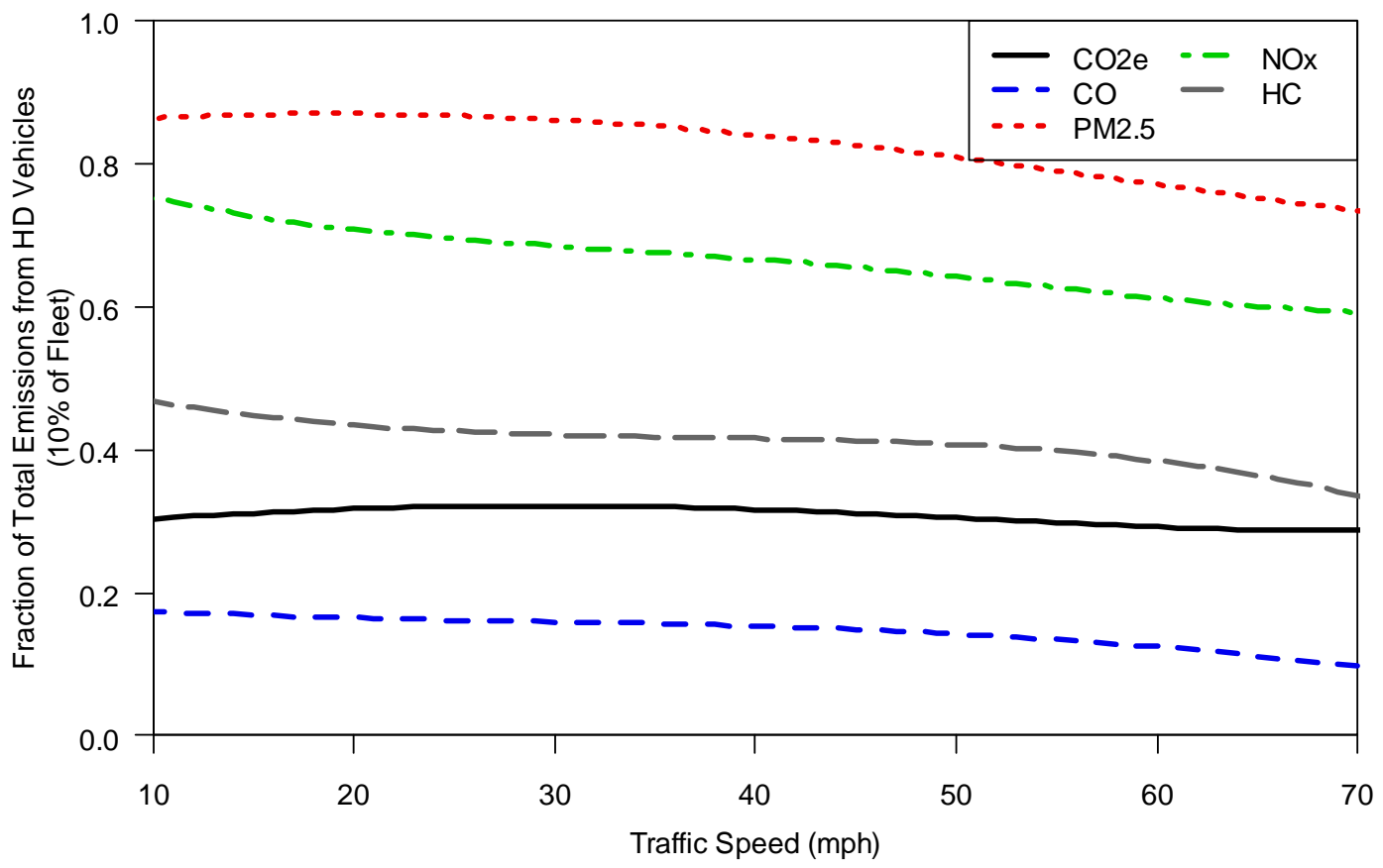

Figure 26. Fraction of Total Emissions from HD Vehicles, $\frac{E_{h}}{E}$, versus $\bar{v}\left(f_{h}=0.1\right)$

The different contributions to total emissions from each vehicle class in Figure 26 weight each vehicle class's effect on $\varepsilon_{E}^{\bar{v}}$ (see Equation 27). As shown by Equation 53, if the travel speed $\bar{v}$ and true demand elasticity $\eta_{q_{j}}^{v_{j}}$ put us between the two curves in Figure 24 and Figure 25, then emissions break-even conditions can still exist when $\frac{E_{l}}{E_{h}}=\frac{-\varepsilon_{E_{h}}^{v_{h}}}{\varepsilon_{E_{l}}^{v_{l}}}$. Since $\varepsilon_{E_{j}}^{v_{j}}$ is the vertical distance between $\eta_{q_{j}}^{v_{j}}$ and $\gamma_{q_{j}}^{v_{j}}$ (Equation 39), the proportions shown in Figure 26 are also the fractions of the vertical distance between $\gamma_{q_{h}}^{v_{h}}$ and $\gamma_{q_{l}}^{v_{l}}$ in Figure 24 and Figure 25 where total emissions break-even conditions exist when $\eta_{q_{h}}^{v_{h}}=\eta_{q_{l}}^{v_{l}}$. Thus, for $f_{h}=0.1$ and similar $\eta_{q_{j}}^{v_{j}}$, the combined emissions break-even curve is closer to $\gamma_{q_{h}}^{v_{h}}$ for $\mathrm{PM}_{2.5}$ and $\mathrm{NO}_{\mathrm{x}}$ and closer to $\gamma_{q_{l}}^{v_{l}}$ for the other pollutants. 
But in addition to different $\gamma_{q_{j}}^{v_{j}}$, LD and HD vehicles are also likely to have different $\eta_{q_{j}}^{v_{j}}$ in a given situation (Graham \& Glaister, 2004). Since travel time costs are a much smaller portion of total travel costs for freight than for personal travel (Graham \& Glaister, 2004; HLB Decision Economics Inc., 2008), it is possible that freight travel demand is less sensitive to travel time costs than passenger travel demand (though this has not yet been empirically demonstrated, to the author's knowledge). If HD vehicle travel demand (primarily goods movements) is less elastic to travel speed than LD vehicle travel demand, $\eta_{q_{h}}^{v_{h}}<\eta_{q_{l}}^{v_{l}}$, then the potential for HD vehicle emissions reductions through speed increases improves.

As an illustration, Figure 27 shows the elasticity of total $\mathrm{CO}_{2} \mathrm{e}$ emissions to uniform travel speed changes, $\varepsilon_{E}^{\bar{v}}$ (computed from Equation 25), on the $\eta_{q_{l}}^{v_{l}}$ versus $\bar{v}$ plane, assuming $f_{H}=0.1$ and $v_{l}=v_{h}=\bar{v}$. The three panels in Figure 27 present the results assuming (a) $\eta_{q_{h}}^{v_{h}}=\eta_{q_{l}}^{v_{l}}$, (b) $\eta_{q_{h}}^{v_{h}}=\eta_{q_{l}}^{v_{l}} \cdot 50 \%$ and (c) $\eta_{q_{h}}^{v_{h}}=0$, in accordance with the wide range found in the literature (see Table 2). Although HD vehicles makeup only $10 \%$ of the fleet, reducing $\eta_{q_{h}}^{v_{h}}$ substantially increases the potential emissions benefits of general travel speed increases. These results are for $\mathrm{CO}_{2} \mathrm{e}$ emissions, of which about $30 \%$ come from HD vehicles (Figure 26). The impact is 2 to 3 times greater for $\mathrm{PM}_{2.5}$ since HD vehicles dominate those emissions. 


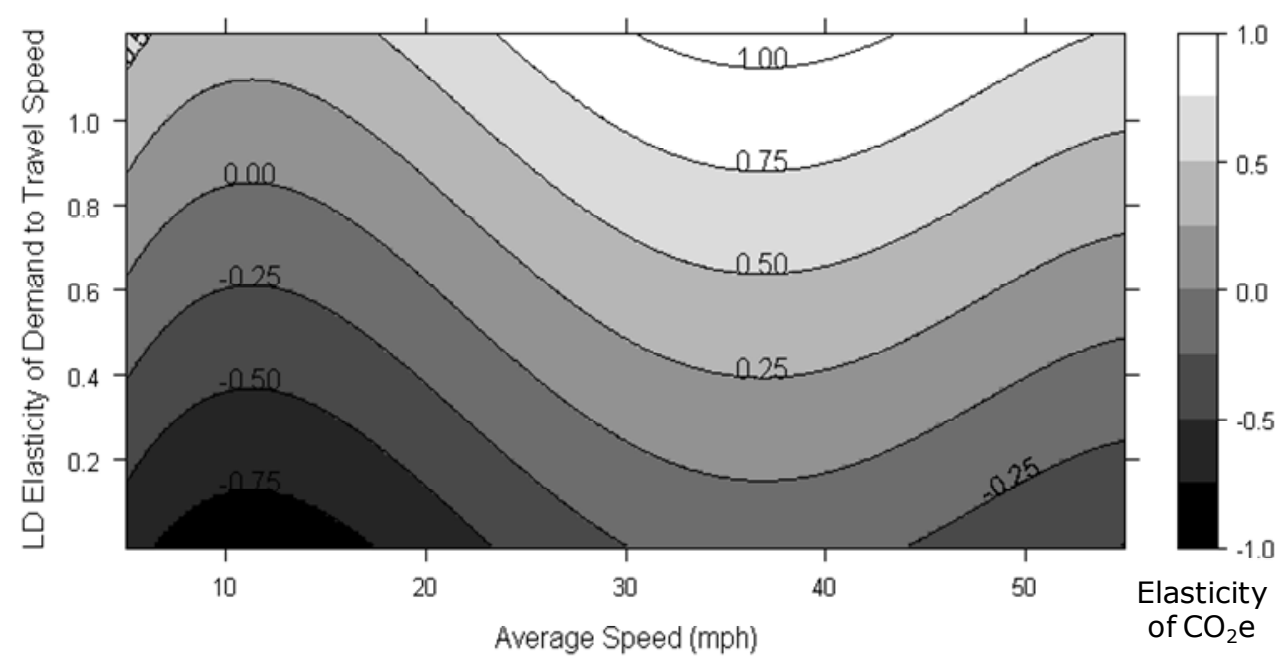

(a)

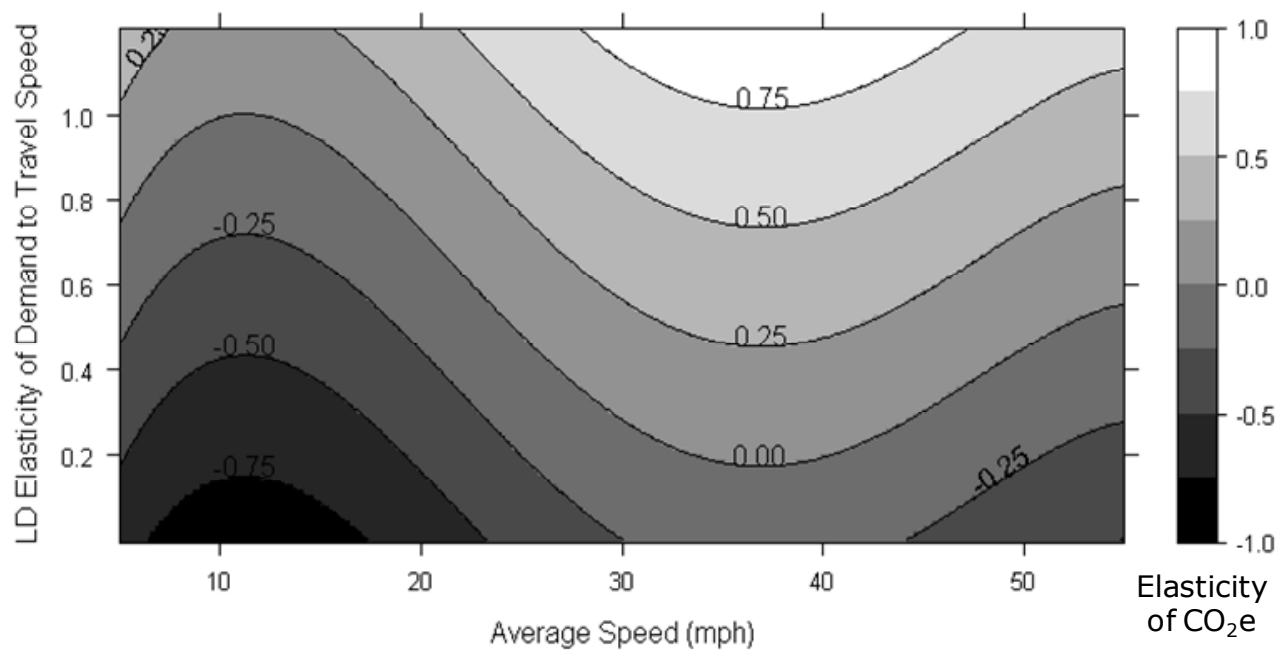

(b)

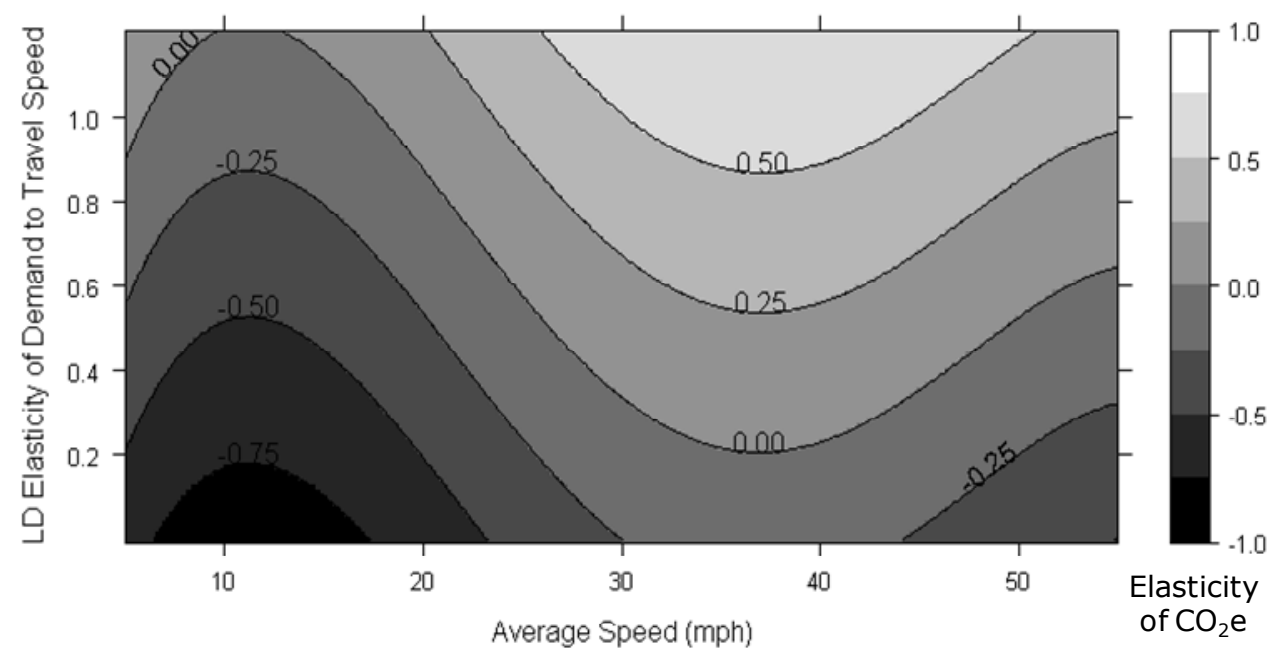

(c)

Figure 27. $\mathrm{CO}_{2} \mathrm{e}$ Emissions Elasticity to Average Speed, $\varepsilon_{E}^{\bar{v}}$, with $\boldsymbol{f}_{\boldsymbol{h}}=0.1$ and $\boldsymbol{\eta}_{q_{h}}^{v_{h}}$ at (a) $100 \%$, (b) $50 \%$, and (c) $0 \%$ of $\eta_{q_{l}}^{v_{l}}$ 
In conclusion, heavy-duty vehicles contribute a large share of on-road emissions, particularly for $\mathrm{PM}_{2.5}$ and $\mathrm{NO}_{\mathrm{x}}$, even though they are the minority of vehicles the fleet. Heavy vehicles also are more sensitive to speed than light-duty vehicles, which leads to higher break-even demand elasticities for heavy-duty vehicles and potentially greater emissions benefits from congestion mitigation. In the next chapter we build on the results of Chapters 5, 6, and 7 to estimate the emissions effects of congestion mitigation, and compare them with other emissions-reduction strategies. 


\section{EMISSIONS IMPACTS OF CONGESTION MITIGATION}

This chapter discusses how the above results can illuminate the emissionsreducing potential of congestion mitigation. We compare the likely benefits of capacitybased strategies (CBS) and non-capacity-based strategies (NCBS) for emissions reductions. The base conditions are an all-ICE fleet with both LD and HD vehicles. Further assumptions are described below.

\subsection{Capacity-based Congestion Mitigation}

\subsubsection{Local Emissions Effects of Congestion Reduction through Capacity Expansion}

At the link level, if there is congestion there are queued or delayed vehicles upstream, so traffic flow or capacity changes that increase very-low speeds involve increasing flow rates (see Figure 12 for speeds below $30 \mathrm{mph}$ ). Depending on the initial traffic state, this flow increase could reduce or overturn emissions rate benefits from increased speeds. The total emissions over the roadway corridor over the peak period might be lower because of lower marginal emissions rates (depending on the demand elasticity), but the speed increase would provoke a spatial-temporal relocation of emissions to the formerly congested section - which could be important for exposure and hot-spot analyses. For local pollutants in urban areas, the location of congestion and its proximity to dense or sensitive populations is an essential consideration.

\subsubsection{Total Emissions Effects of Congestion Reduction through Capacity Expansion}

The results in Section 5.5 for total emissions elasticity to speed changes illustrate the emissions impacts of CBS. For CBS with no demand constraints the total emissions 
effects can be estimated using expected values for demand elasticity, $\eta_{q_{j}}^{v_{j}}$. If the true demand elasticity is highly uncertain, a simple increase/decrease estimate can be made using the break-even demand elasticity and a likely range of true elasticities. If it is reasonably expected that $\eta_{q_{j}}^{v_{j}}>\gamma_{q_{j}}^{v_{j}}$ then CBS will likely increase total emissions. The results in previous sections show potential total emissions reductions from CBS only for low demand elasticities with moderate existing congestion levels or moderate demand elasticities with heavy existing congestion. These results, however, can vary by pollutant and vehicle class.

Figure 28 shows characterizations of CBS for each pollutant over a range of speeds. The characterizations are based on ranges of break-even demand elasticity, $\gamma_{q_{j}}^{v_{j}}$ : CBS are "not recommended" for $\gamma_{q_{j}}^{v_{j}}<0.25$, are suggested to "apply with caution" for $0.25 \leq \gamma_{q_{j}}^{v_{j}}<0.5$, have "potential benefits" for $0.5 \leq \gamma_{q_{j}}^{v_{j}}<0.75$, and provide "good opportunity" for emissions reductions for $0.75 \leq \gamma_{q_{j}}^{v_{j}}$. These are subjective, qualitative labels based on the literature reviewed in Section 2.5.

Clearly $\mathrm{PM}_{2.5}$ and $\mathrm{HC}$ have the widest range of speeds for which CBS are likely to reduce emissions. The other pollutants are only classified as "potential benefits" at speeds around 20 and below. CBS are "not recommended" for all pollutants at speeds above $65 \mathrm{mph}$, which shows the potential benefits of limiting free-flow speeds to below $65 \mathrm{mph}$. 


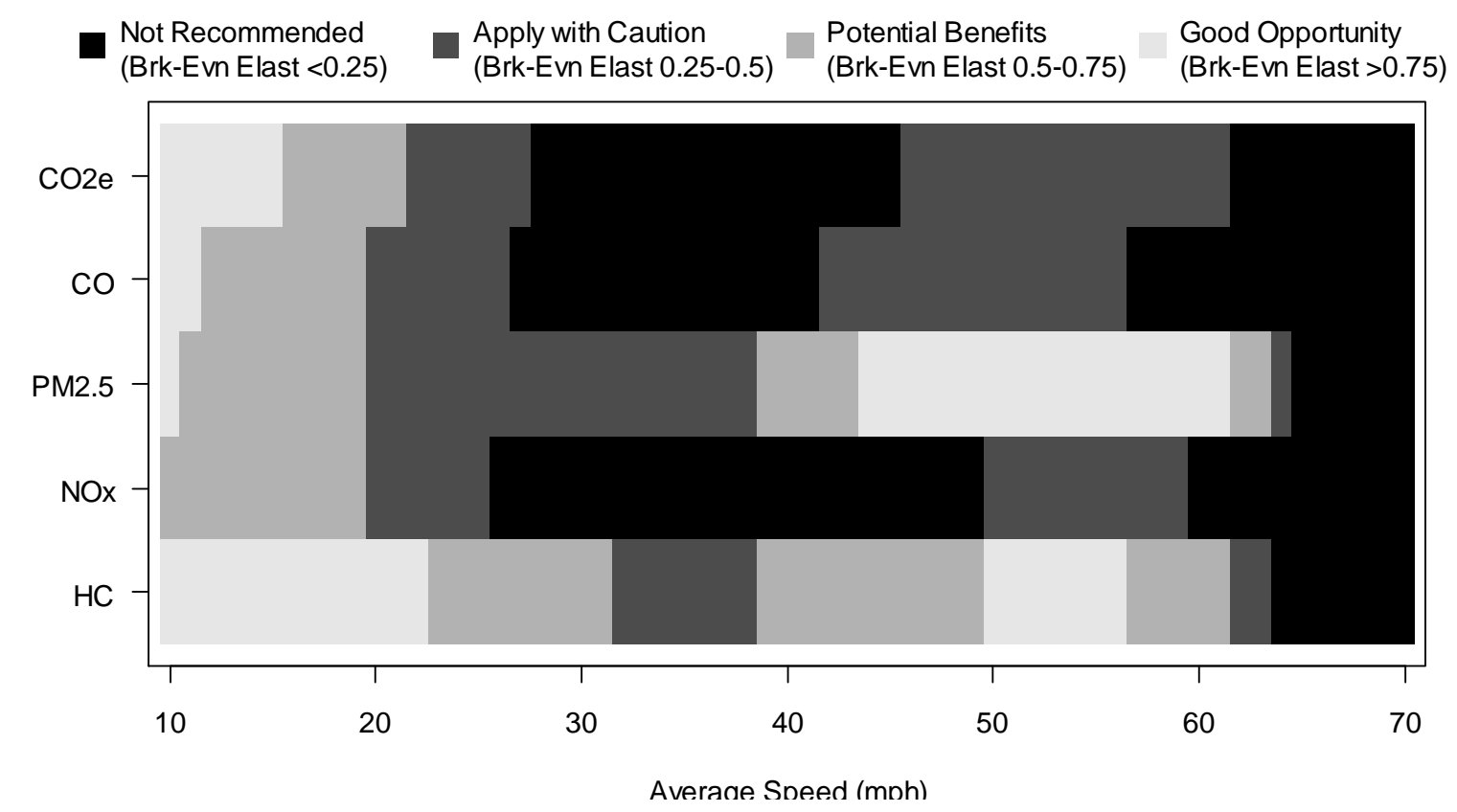

Figure 28. Characterization of CBS Based on Ranges of Break-Even Elasticities, $\gamma_{q_{j}}^{v_{j}}$

As a further demonstration of the impacts of HD vehicle demand elasticity shown in Figure 27, Figure 29 shows the same characterization of CBS, but assuming the extreme case of $\eta_{q_{h}}^{v_{h}}=0$. We also assume $f_{h}=0.1$, as in Figure 27. Here there is a wider range of speeds for all pollutants which present opportunities for emissions reductions through CBS. For PM2.5 and HC good opportunities exist for emissions reductions through CBS from $10 \mathrm{mph}$ all the way up beyond $60 \mathrm{mph}$. Although this is an extreme value of demand elasticity for HD vehicles, it demonstrates that even at only $10 \%$ of the fleet, $\eta_{q_{h}}^{v_{h}}$ is an important consideration for predicting emissions effects of congestion mitigation. 


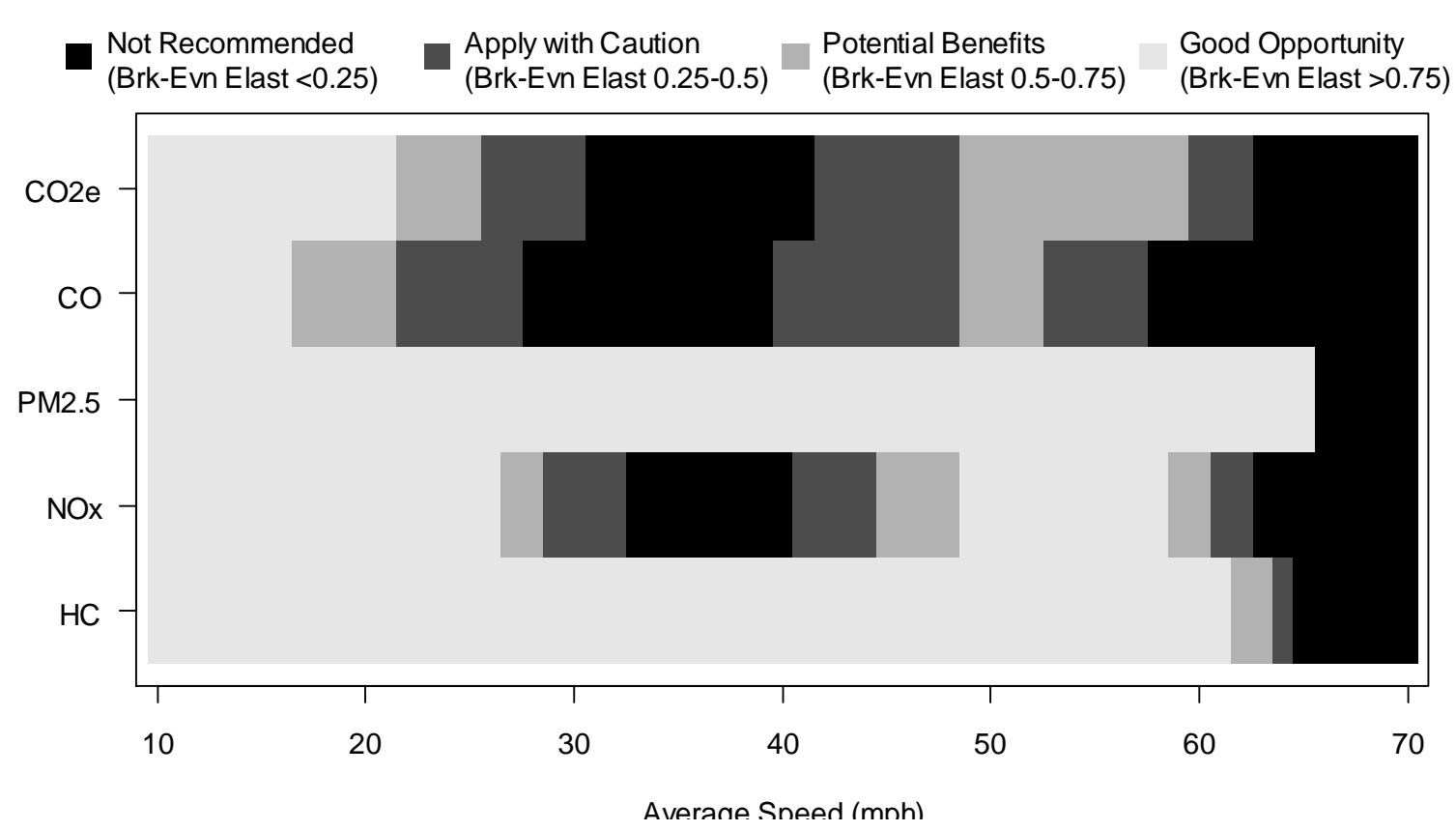

Figure 29. Characterization of CBS Based on Ranges of Break-Even Elasticities for LD Vehicles, $\gamma_{q_{l}}^{v_{l}}$, Assuming $\eta_{q_{h}}^{v_{h}}=0$ and $f_{h}=0.1$

As a final note, CBS are not necessarily additional lane-miles. Capacity or throughput can also be increased by various traffic management strategies that target roadway efficiency such as variable speed limits on freeways or traffic signal coordination on arterials. Some traffic management techniques would have implications for speed profiles (drive schedules) that would affect average-speed emissions rate estimates and so change the ESC used to derive $\gamma_{q_{j}}^{v_{j}}$. For example, a significant "smoothing" of vehicle speeds could reduce the average emissions rate at a given average travel speed by reducing engine loads (Barth \& Boriboonsomsin, 2008). This change in the ESC would have to be considered in concert with average travel speed and travel demand changes to estimate the full emissions impact of congestion-relieving CBS through roadway/traffic management. 


\subsection{Travel Demand Reductions}

When $\eta_{q_{j}}^{v_{j}}>\gamma_{q_{j}}^{v_{j}}$ for all vehicle classes $j$, speed-based efficiency alone cannot reduce total emissions and some demand restraint must be employed if we want to mitigate emissions. From another perspective, when $\eta_{q_{j}}^{v_{j}}>\gamma_{q_{j}}^{v_{j}}$ a capacity decrease (i.e. "road diets") would likely reduce total emissions because the suppressed demand volume would offset engine inefficiencies at lower speeds. In other words, with a capacity-based approach, lower total emissions are more likely by increasing capacity when $\eta_{q_{j}}^{v_{j}}<\gamma_{q_{j}}^{v_{j}}$ and by decreasing capacity when $\eta_{q_{j}}^{v_{j}}>\gamma_{q_{j}}^{v_{j}}$. This, of course, assumes that demand elasticities to speed changes in each direction are the same - i.e. the aggregate travel response to a speed increase is equal and opposite to the response to a speed decrease. Using arc elasticities as in Equation 12, this isotropic nature of demand elasticity can be $\operatorname{expressed} \eta_{q_{j}}^{v_{j}}=\frac{\left(v_{j_{2}}+v_{j_{1}}\right)\left(q_{j_{2}}-q_{j_{1}}\right)}{\left(q_{j_{2}}+q_{j_{1}}\right)\left(v_{j_{2}}-v_{j_{1}}\right)}=\frac{\left(v_{j_{2}}+v_{j_{1}}\right)\left(q_{j_{1}}-q_{j_{2}}\right)}{\left(q_{j_{2}}+q_{j_{1}}\right)\left(v_{j_{1}}-v_{j_{2}}\right)}$

There are, additionally, NCBS where travel demand is reduced by motivators other than travel time increases (road pricing or travel restrictions, for example). In those situations the key value for application of these analysis tools is the net demand elasticity to travel speed: the net change in travel demand with changes in travel speed, after adjusting for the NCBS. For example, if a demand-moderating measure (such as road pricing) is implemented along with a capacity expansion, then that can be incorporated into the estimate of expected $\eta_{q_{j}}^{v_{j}}$. When $\eta_{q_{j}}^{v_{j}}>\gamma_{q_{j}}^{v_{j}}$ and emissions increases are a likely result of capacity-based congestion mitigation, road pricing presents an option for decreasing or reversing that effect. 


\subsection{Comparing Strategies for Emissions Reductions}

In this section we put emissions changes from speed improvements into context by rough comparison with a set of alternative NCBS for emissions improvements. We are evaluating changes in total emissions from peak period travel for highly aggregate conditions. The alternative strategies considered are

1. reduced VMT as reflected by peak-period VMT per peak-period traveler (made possible by denser, more mixed land use, road pricing, or other demand management strategies),

2. vehicle fleet fuel efficiency improvements (by lighter vehicles or less powerintensive engines),

3. reduced fuel carbon intensity (by using alternative fuels such as biodiesel or electricity, or by less energy-intensive fuel production and delivery methods), and

4. replacement of light-duty ICE vehicles in the fleet with electric vehicles.

For travel distance reduction (1) the net VMT change is assumed to be reflected in a change in average peak-period VMT per peak period traveler - accounting for potential demand rebound due to travel time savings and assuming a fixed number of peak period travelers. We further assume that the VMT reduction has no net effect on average emissions rates (which is the case if there is no change in $q$ on congested facilities). In reality, the VMT reduction could have varying effects on $q$ (and $\bar{e}$ ) depending on how it is achieved. Demand management which targets the number of trips would likely reduce $q$ (thus increasing $\bar{v}$ and reducing $\bar{e}$ ). Land use strategies which encourage shorter trips through increased density would more likely increase $q$ (thus decreasing $\bar{v}$ and increasing 
$\bar{e})$. Also, there could be a shift in travel in which $q$ increases or decreases with no net effect on VMT - such as a partial VMT rebound from shorter trips increasing $q$ or a partial VMT rebound from lower $q$ as longer trips. Regardless of the effect of (1) on $q$, the assumption of $\bar{e}$ insensitivity to VMT reductions is likely to be sound since the elasticity of emissions rates to volume, $\varepsilon_{\bar{e}}^{q}$, is low for a wide range of $q$ (see Section 5.7).

The vehicle-based NCBS (2-4) do not increase capacity or improve traffic flow and therefore we assume that there is no speed-induced demand generated by their application. It is worth noting that increasing fuel efficiency (2) can reduce operational costs and there is potential for induced demand through travel cost reductions. Similarly, depending on the costs of EV's and electricity, there could be marginal operating cost reductions for EV replacement of LD ICE vehicles (4) which induces demand. This effect, like the induced demand effect from travel time savings, could be offset by additional pricing of travel or fuel.

The main assumptions used for this comparison analysis are:

1. no additional demand volume or average speed changes are generated by application of the NCBS,

2. average daily peak period travel on freeway and arterial facilities is 8.0 and 8.6 miles, respectively, per peak period traveler (the average of 439 U.S. urban areas in 2007 - extractable from the data tables accompanying the Urban Mobility Report (UMR) (Schrank \& Lomax, 2009)),

3. $55 \%$ of peak period freeway and arterial travel (by VMT) is congested (the average of 439 U.S. urban areas in 2007 - from the UMR data tables), 
4. average fuel efficiency of 21.1 miles per gallon (mpg) (for the U.S. light-duty vehicle fleet, model year 2009, from the U.S. EPA (2009b)),

5. average fuel carbon intensity of $8.9 \mathrm{kgCO}_{2} \mathrm{e}$ per gallon (calculated from U.S. EPA, 2009b),

6. electric vehicle carbon intensity of travel of $0.216 \mathrm{kgCO}_{2}$ e per mile (from the supplementary material of Samaras \& Meisterling (2008)), and

7. all other fleet and emissions characteristics are as described above, as modeled in MOVES for Portland, 2010.

The EV carbon intensity of travel is based on life-cycle assessment (LCA), although upstream emissions are not included in the roadway emissions estimates for petroleum vehicles. In order to make an equivalent comparison with the on-road emissions estimates, an additional estimate is made using zero emissions for EV's. The assumption of zero emissions for EV's is also made for local pollutants (all non- $\mathrm{CO}_{2}$ ), since EV's produce no on-road emissions (though they do contribute to regional air pollution through power generation).

In this analysis we use the emissions rates generated by MOVES modeling for a full fleet and a LD-only fleet (for the EV replacements). The calculations for VMT reductions (1) and EV penetration (4) use average emissions rates by facility (including congested and uncongested conditions); calculations for the two fuel-based NCBS, (2) and (3), use the average fuel economy and carbon intensity given in the list of assumptions above. The portion of peak-period travel on uncongested freeways and arterials is assumed to have average speeds of $60 \mathrm{mph}$ and $35 \mathrm{mph}$, respectively. Emissions from travel on local roads is neglected - a conservative assumption with 
respect to the NCBS. Induced demand is calculated using mid-point arc elasticity as in Equation 12.

We first look at freeway facilities alone, comparing NCBS to CBS that increase congested speeds as indicated by improving freeway LOS. The results of this comparison for freeway $\mathrm{CO}_{2} \mathrm{e}$ are shown in Table 9 and Table 10 using demand elasticities to travel speed of 0.0 and 0.3 , respectively. The three numerical columns (from left to right) show LOS changes from $\mathrm{F}$ to $\mathrm{E}$, from $\mathrm{E}$ to $\mathrm{D}$, and from $\mathrm{D}$ to the $\mathrm{A}-\mathrm{C}$ range (again, LOS average speeds are from Barth et al. (1999)). Only emissions from freeway travel are considered here, and the LOS change only applies to the congested portion of freeway travel (55\%). The NCBS effects apply to all peak-period freeway travel, but other impacts are excluded (e.g. EV ownership would also reduce emissions from non-peak period trips and from travel on non-freeway facilities). The table results also assume independence of strategies - in other words changes to travel distance or vehicle efficiency do not affect travel speeds. For each hypothetical LOS improvement the net changes in average speed, travel demand volume, and commute emissions are shown in the first three rows of the Table. The final rows show the NCBS changes that would be required to generate the same peak period emissions change on freeway facilities from each alternative strategy. 
Table 9. Comparison of Equivalent Emissions Reduction Strategies for Freeway $\mathrm{CO}_{2} \mathrm{e}$ Emissions (MOVES Model with Inelastic Demand, $\varepsilon_{q}^{\bar{v}}=0$ )

\begin{tabular}{|c|c|c|c|}
\hline & 19 - $31 \mathrm{mph}$ & $31-53 \mathrm{mph}$ & $53-60 \mathrm{mph}$ \\
\hline Avg. speed change (mph) & $11.9(64 \%)$ & $22.4(73 \%)$ & $6.8(13 \%)$ \\
\hline $\begin{array}{l}\text { Travel demand change } \\
\text { (vehicle miles/peak-traveler-day) }\end{array}$ & 0 & 0 & 0 \\
\hline $\begin{array}{l}\text { Emissions change } \\
\qquad\left(\mathrm{g} \mathrm{CO}_{2} \mathrm{e} / \text { peak-traveler-day }\right)\end{array}$ & $-481(-11 \%)$ & $-236(-6 \%)$ & $-101(-3 \%)$ \\
\hline \multicolumn{4}{|l|}{ Alternative Efficiency Strategy } \\
\hline $\begin{array}{l}\text { Trips length change } \\
\text { (vehicle miles/peak-traveler-day) }\end{array}$ & $-0.9(-11 \%)$ & $-0.5(-6 \%)$ & $-0.2(-3 \%)$ \\
\hline $\begin{array}{l}\text { Vehicle efficiency change } \\
\text { (miles/gallon) }\end{array}$ & $2.1(13 \%)$ & $1.3(7 \%)$ & $0.6(3 \%)$ \\
\hline $\begin{array}{l}\text { Fuel carbon intensity change } \\
\left(\mathrm{kg} \mathrm{CO}_{2} \mathrm{e} / \text { gallon }\right)\end{array}$ & $-1.0(-11 \%)$ & $-0.6(-6 \%)$ & $-0.3(-3 \%)$ \\
\hline $\begin{array}{l}\text { EV penetration by LCA } \\
\text { (\% of peak period fleet) }\end{array}$ & $29 \%$ & $19 \%$ & $9 \%$ \\
\hline $\begin{array}{l}\text { EV penetration by zero-emissions } \\
\text { (\% of peak period fleet) }\end{array}$ & $14 \%$ & $8 \%$ & $4 \%$ \\
\hline
\end{tabular}

Table 10. Comparison of Equivalent Emissions Reduction Strategies for freeway

$\mathrm{CO}_{2} \mathrm{e}$ Emissions (MOVES Model with $\varepsilon_{q}^{\bar{v}}=0.3$ )

\begin{tabular}{|c|c|c|c|}
\hline & $19-31 \mathrm{mph}$ & $31-53 \mathrm{mph}$ & $53-60 \mathrm{mph}$ \\
\hline Avg. speed change (mph) & $11.9(64 \%)$ & $22.4(73 \%)$ & $6.8(13 \%)$ \\
\hline $\begin{array}{l}\text { Travel demand change } \\
\text { (vehicle miles/peak-traveler-day) }\end{array}$ & $0.7(9 \%)$ & $0.8(10 \%)$ & $0.2(2 \%)$ \\
\hline $\begin{array}{l}\text { Emissions change } \\
\qquad\left(\mathrm{g} \mathrm{CO}_{2} \mathrm{e} / \text { peak-traveler-day }\right)\end{array}$ & $-131(-3 \%)$ & $112(3 \%)$ & $-31(-1 \%)$ \\
\hline \multicolumn{4}{|l|}{ Alternative Efficiency Strategy } \\
\hline $\begin{array}{l}\text { Trips length change } \\
\text { (vehicle miles/peak-traveler-day) }\end{array}$ & $-0.2(-3 \%)$ & $0.2(3 \%)$ & $-0.1(-1 \%)$ \\
\hline $\begin{array}{l}\text { Vehicle efficiency change } \\
\text { (miles/gallon) }\end{array}$ & $0.5(3 \%)$ & $-0.5(-3 \%)$ & $0.2(1 \%)$ \\
\hline $\begin{array}{l}\text { Fuel carbon intensity change } \\
\qquad\left(\mathrm{kg} \mathrm{CO}_{2} \mathrm{e} / \text { gallon }\right)\end{array}$ & $-0.3(-3 \%)$ & $0.3(3 \%)$ & $-0.1(-1 \%)$ \\
\hline $\begin{array}{l}\text { EV penetration by LCA } \\
\text { (\% of peak period fleet) }\end{array}$ & $8 \%$ & $-9 \%$ & $3 \%$ \\
\hline $\begin{array}{l}\text { EV penetration by zero-emissions } \\
\text { ( } \% \text { of peak period fleet) }\end{array}$ & $4 \%$ & $-4 \%$ & $1 \%$ \\
\hline
\end{tabular}


As an example, consider the first numerical column of Table 10, which considers $\mathrm{CO}_{2} \mathrm{e}$ emissions from a freeway LOS change from $\mathrm{F}$ to $\mathrm{E}$. The average speed change on congested freeways from 19 to $31 \mathrm{mph}$ (rounded) is a speed increase of $11.9 \mathrm{mph}$ (64\%) - row 1. Assuming $\varepsilon_{q}^{\bar{v}}=0.3$ in this table, this speed increase induces 0.7 extra vehiclemiles of peak period freeway travel (per day per peak period traveler), an increase of $9 \%$ - row 2. Considering the increased efficiency and induced demand, total emissions are reduced by 131 grams per peak period traveler, per day $(-3 \%)$ - row 3 . This 131 grams of emissions savings could also have been achieved by reducing daily peak-period freeway travel by 0.2 vehicle-miles per peak period traveler (-3\%) - row 4. Alternatively, 131 grams of $\mathrm{CO}_{2}$ e could be saved if daily peak-period freeway travel were in vehicles with $0.5 \mathrm{mpg}$ better fuel economy on average (3\%) - row 5 . A decrease of $0.3 \mathrm{~kg} \mathrm{CO}_{2} \mathrm{e}$ per gallon $(-3 \%)$ in the carbon intensity of fuel burned during peak-period freeway travel could also save 131 grams of $\mathrm{CO}_{2} \mathrm{e}$ emissions - row 6. Finally, replacing 8\% (by LCA) or 4\% (by zero-emissions EV's) of ICE LD vehicles with EV's for peak-period freeway travel could also achieve the same savings of 131 grams $\mathrm{CO}_{2} \mathrm{e}-$ rows $7 \& 8$.

As expected from the previous modeling in this thesis, the LOS change from $\mathrm{F}$ to E generates the greatest marginal benefits, which require the largest alternative efficiency improvements to match. The greatest difference between speed improvement and emissions reduction is observed in the central column, LOS E to D. With inelastic demand (Table 9) a 73\% increase in freeway speed garners a meager $6 \%$ in terms of emissions reductions. Similar reductions can be achieved by increasing fleet fuel efficiency by $1.3 \mathrm{mpg}$ or reducing average peak period freeway travel by half a mile per peak period traveler, per day. Furthermore, alternative strategies have the potential for 
low or zero costs for transportation agencies, while capital improvement projects such as urban freeway widening can be extremely expensive endeavors. These results are relatively conservative, since they are only considering peak-period travel on freeways. The fuel-related and EV-related NCBS would have additional benefits from travel throughout the day on all facilities.

The values in Table 9 are based on the MOVES-modeled emissions rates. A comparable table based on the Barth model is similar for LOS F to E, but the efficiency gains from LOS E to D are less (2\% net emissions reduction). For an improvement from LOS D to the LOS A-C range the Barth model predicts net emissions increases (even with inelastic demand) because of the inefficiency of high-speed travel. The Boulter model produces even smaller efficiency gains, with net emissions changes of $-9 \%,-1 \%$, and $8 \%$ for the three columns in Table 9.

The results in Table 10 incorporate induced demand with an assumed elasticity of travel demand to travel speed of 0.3 . The speed improvements reduce primary-road travel time and so induce travel that partially or fully offsets the emissions rate reductions seen in Table 9. The emissions changes shown in row three of Table 10 include both the emissions rate and induced travel effects. Even with moderate elasticity $\left(\varepsilon_{q}^{\bar{v}}=0.3\right)$ the emissions savings in columns 1 and 3 are less than half as large as in Table 9, while the induced travel for LOS E to LOS D leads to a total emissions increase. When a total emissions increase is expected, the alternative strategy equivalents have opposite signs from an emissions savings - i.e. longer trips, reduced vehicle efficiency, higher fuel carbon intensity, and fewer EV's in the fleet. Using an assumed elasticity of 0.5 the induced travel leads to total emission increases for all three LOS improvements. 
Table 11 shows the results of a similar analysis (with the same assumptions) for $\mathrm{CO}_{2} \mathrm{e}$ emissions on arterials with an assumed demand elasticity of 0.3 . Here we use travel speed increases of 10 to $16 \mathrm{mph}, 16$ to $24 \mathrm{mph}$, and 24 to $35 \mathrm{mph}$, roughly parallel to the heavily congested - moderately congested - uncongested LOS improvements in the freeway tables. As expected for a lower-speed facility and from the emissions gradients in Figure 7, arterial congestion mitigation is more effective at reducing emissions. Still, even with moderate demand elasticity $\left(\varepsilon_{q}^{\bar{v}}=0.3\right)$ the speed improvement above 24 mph produces a net emissions increase because of induced demand.

Table 11. Comparison of Equivalent Emissions Reduction Strategies for Arterial $\mathrm{CO}_{2} \mathrm{e}$ Emissions (MOVES Model with $\varepsilon_{q}^{\bar{v}}=0.3$ )

\begin{tabular}{|c|c|c|c|}
\hline & $10-16 \mathrm{mph}$ & $16-24 \mathrm{mph}$ & $24-35 \mathrm{mph}$ \\
\hline Avg. speed change (mph) & $6.0(60 \%)$ & $8.0(50 \%)$ & $11.0(46 \%)$ \\
\hline $\begin{array}{l}\text { Travel demand change } \\
\text { (vehicle miles/peak-traveler-day) }\end{array}$ & $0.7(9 \%)$ & $0.6(8 \%)$ & $0.6(7 \%)$ \\
\hline $\begin{array}{l}\text { Emissions change } \\
\quad\left(\mathrm{g} \mathrm{CO}_{2} \mathrm{e} / \text { peak-traveler-day }\right)\end{array}$ & $-1,002(-15 \%)$ & $-374(-7 \%)$ & $31(1 \%)$ \\
\hline \multicolumn{4}{|l|}{ Alternative Efficiency Strategy } \\
\hline $\begin{array}{l}\text { Trips length change } \\
\text { (vehicle miles/peak-traveler-day) }\end{array}$ & $-1.3(-15 \%)$ & $-0.6(-7 \%)$ & $0.1(1 \%)$ \\
\hline $\begin{array}{l}\text { Vehicle efficiency change } \\
\text { (miles/gallon) }\end{array}$ & $1.9(17 \%)$ & $1.1(8 \%)$ & $-0.1(-1 \%)$ \\
\hline $\begin{array}{l}\text { Fuel carbon intensity change } \\
\left.\text { ( } \mathrm{kg} \mathrm{CO}_{2} \mathrm{e} / \text { gallon }\right)\end{array}$ & $-1.3(-15 \%)$ & $-0.6(-7 \%)$ & $0.1(1 \%)$ \\
\hline $\begin{array}{l}\text { EV penetration by LCA } \\
\text { (\% of peak period fleet) }\end{array}$ & $29 \%$ & $17 \%$ & $-2 \%$ \\
\hline $\begin{array}{l}\text { EV penetration by zero-emissions } \\
\text { (\% of peak period fleet) }\end{array}$ & $19 \%$ & $9 \%$ & $-1 \%$ \\
\hline
\end{tabular}

The next four tables show the same efficiency strategy comparisons on freeways and arterials for $\mathrm{NO}_{\mathrm{x}}$ (Table 12 and Table 13) and $\mathrm{PM}_{2.5}$ (Table 14 and Table 15) using 
demand elasticity of $\varepsilon_{q}^{\bar{v}}=0.3$. The largest emissions reductions are for heavily congested arterials. $\mathrm{NO}_{\mathrm{x}}$ emissions show no benefit from freeway congestion mitigation. Unlike the other pollutant-facility combinations, freeway $\mathrm{PM}_{2.5}$ emissions have the largest potential savings from a reduction of moderate congestion. This is also reflected in the high breakeven demand elasticity at moderate speeds for $\mathrm{PM}_{2.5}$ in Figure 18. The EV penetration of the LD vehicle fleet must be particularly high to match emissions reductions from speed improvements for $\mathrm{PM}_{2.5}$. This is logical, because the EV's are only replacing LD vehicles and the $\mathrm{PM}_{2.5}$ emissions are primarily from the HD portion of the fleet (see Figure 26).

Collectively, these tables show that considering moderate values for demand elasticity substantially degrades the potential for emissions reductions from CBS and increases the attractiveness of alternative strategies. That said, there are still some situations where traffic flow improvements can substantially reduce emissions, such as heavily congested arterials. But CBS for emissions reductions are still not likely to be the most cost-effective approach, considering the potential for low-capital-cost alternative efficiency strategies. CBS are more susceptible to self-defeating behavior responses through induced travel, and only provide efficiency benefits during peak periods. Finally, elasticities higher than 0.3 - which are realistic on long time scales - will increase total emissions in many CBS situations. 
Table 12. Comparison of Equivalent Emissions Reduction Strategies for Freeway NO $\mathrm{x}_{\mathrm{x}}$ Emissions (MOVES Model with $\varepsilon_{q}^{\bar{v}}=0.3$ )

\begin{tabular}{|c|c|c|c|}
\hline & $\mathbf{1 9 - 3 1} \mathbf{~ m p h}$ & $\mathbf{3 1} \mathbf{- 5 3} \mathbf{~ m p h}$ & $\mathbf{5 3 - 6 0} \mathbf{~ m p h}$ \\
\hline Avg. speed change (mph) & $11.9(64 \%)$ & $22.4(73 \%)$ & $6.8(13 \%)$ \\
\hline $\begin{array}{c}\text { Travel demand change } \\
\text { (vehicle miles/peak-traveler-day) }\end{array}$ & $0.7(9 \%)$ & $0.8(10 \%)$ & $0.2(2 \%)$ \\
\hline $\begin{array}{c}\text { Emissions change } \\
\text { (g CO } \mathrm{e}_{2} \text { /peak-traveler-day) }\end{array}$ & $-0.1(-1 \%)$ & $0.5(6 \%)$ & $0.0(0 \%)$ \\
\hline Alternative Efficiency Strategy & $-0.0(-1 \%)$ & $0.5(6 \%)$ & $0.0(0 \%)$ \\
\hline $\begin{array}{c}\text { Trips length change } \\
\text { (vehicle miles/peak-traveler-day) }\end{array}$ & $1 \%$ & $-14 \%$ & $0 \%$ \\
\hline $\begin{array}{c}\text { EV penetration by zero-emissions } \\
\text { (\% of peak period fleet) }\end{array}$ & & & \\
\hline
\end{tabular}

Table 13. Comparison of Equivalent Emissions Reduction Strategies for Arterial NO ${ }_{\mathrm{x}}$ Emissions (MOVES Model with $\varepsilon_{q}^{\bar{v}}=0.3$ )

\begin{tabular}{|c|c|c|c|}
\hline & $\mathbf{1 0 - 1 6} \mathbf{~ m p h}$ & $\mathbf{1 6 - 2 4} \mathbf{~ m p h}$ & $\mathbf{2 4 - 3 5} \mathbf{~ m p h}$ \\
\hline Avg. speed change (mph) & $6.0(60 \%)$ & $8.0(50 \%)$ & $11.0(46 \%)$ \\
\hline $\begin{array}{c}\text { Travel demand change } \\
\text { (vehicle miles/peak-traveler-day) }\end{array}$ & $0.7(9 \%)$ & $0.6(8 \%)$ & $0.6(7 \%)$ \\
\hline $\begin{array}{c}\text { Emissions change } \\
\text { (g CO}{ }_{2} \mathrm{e} / \text { peak-traveler-day) }\end{array}$ & $-1.7(-11 \%)$ & $-0.5(-5 \%)$ & $0.3(2 \%)$ \\
\hline Alternative Efficiency Strategy & $-1.0(-11 \%)$ & $-0.4(-5 \%)$ & $0.2(2 \%)$ \\
\hline $\begin{array}{c}\text { Trips length change } \\
\text { (vehicle miles/peak-traveler-day) }\end{array}$ & $36 \%$ & $13 \%$ & $-7 \%$ \\
\hline $\begin{array}{c}\text { EV penetration by zero-emissions } \\
(\% \text { of peak period fleet) }\end{array}$ & & & \\
\hline
\end{tabular}


Table 14. Comparison of Equivalent Emissions Reduction Strategies for Freeway $\mathbf{P M}_{2.5}$ Emissions (MOVES Model with $\varepsilon_{q}^{\bar{v}}=0.3$ )

\begin{tabular}{|c|c|c|c|}
\hline & $\mathbf{1 9 - 3 1} \mathbf{~ m p h}$ & $\mathbf{3 1} \mathbf{- 5 3} \mathbf{~ m p h}$ & $\mathbf{5 3 - 6 0} \mathbf{~ m p h}$ \\
\hline Avg. speed change (mph) & $11.9(64 \%)$ & $22.4(73 \%)$ & $6.8(13 \%)$ \\
\hline $\begin{array}{c}\text { Travel demand change } \\
\text { (vehicle miles/peak-traveler-day) }\end{array}$ & $0.7(9 \%)$ & $0.8(10 \%)$ & $0.2(2 \%)$ \\
\hline $\begin{array}{c}\text { Emissions change } \\
\text { (g CO } \mathrm{e}_{2} \text { /peak-traveler-day) }\end{array}$ & $-12(-3 \%)$ & $-36(-12 \%)$ & $-14(-6 \%)$ \\
\hline Alternative Efficiency Strategy & $-0.3(-3 \%)$ & $-0.9(-12 \%)$ & $-0.4(-6 \%)$ \\
\hline $\begin{array}{c}\text { Trips length change } \\
\text { (vehicle miles/peak-traveler-day) }\end{array}$ & $18 \%$ & $57 \%$ & $22 \%$ \\
\hline $\begin{array}{c}\text { EV penetration by zero-emissions } \\
\text { (\% of peak period fleet) }\end{array}$ & & \\
\hline
\end{tabular}

Table 15. Comparison of Equivalent Emissions Reduction Strategies for Arterial $\mathbf{P M}_{2.5}$ Emissions (MOVES Model with $\varepsilon_{q}^{\bar{v}}=0.3$ )

\begin{tabular}{|c|c|c|c|}
\hline & $10-16 \mathrm{mph}$ & $16-24 \mathrm{mph}$ & $24-35 \mathrm{mph}$ \\
\hline Avg. speed change (mph) & $6.0(60 \%)$ & $8.0(50 \%)$ & $11.0(46 \%)$ \\
\hline $\begin{array}{l}\text { Travel demand change } \\
\text { (vehicle miles/peak-traveler-day) }\end{array}$ & $0.7(9 \%)$ & $0.6(8 \%)$ & $0.6(7 \%)$ \\
\hline $\begin{array}{l}\text { Emissions change } \\
\quad\left(\mathrm{g} \mathrm{CO}_{2} \mathrm{e} / \text { peak-traveler-day }\right)\end{array}$ & $-74(-12 \%)$ & $-29(-6 \%)$ & $-9(-2 \%)$ \\
\hline \multicolumn{4}{|l|}{ Alternative Efficiency Strategy } \\
\hline $\begin{array}{l}\text { Trips length change } \\
\text { (vehicle miles/peak-traveler-day) }\end{array}$ & $-1.1(-12 \%)$ & $-0.5(-6 \%)$ & $-0.2(-2 \%)$ \\
\hline $\begin{array}{l}\text { EV penetration by zero-emissions } \\
\text { (\% of peak period fleet) }\end{array}$ & $74 \%$ & $37 \%$ & $13 \%$ \\
\hline
\end{tabular}

\subsubsection{Emissions Elasticity to NCBS}

The emissions elasticities to each of these NCBS can be analytically determined. For VMT reductions (1), increased fuel efficiency (2), and decreased carbon intensity (3) the total emissions point elasticity is -1.0. For these strategies we can say that a certain 
percentage emissions reduction from a CBS can also be accomplished through roughly the same percentage implementation of the NCBS. The percent changes for vehicle efficiency (mpg) in Table 9 and Table 11 are slightly different from the emissions savings percentages because emissions are inversely related to efficiency, so the point elasticity of -1.0 will be different from the arc elasticity. For reference, the net percent emissions change from CBS (as described in the previous section) for each facility-pollutant-LOS combination are shown in Table 16 and Table 17, using $\varepsilon_{q}^{\bar{v}}=0.3$. Positive numbers indicate emissions increases. Thus, from the first row of Table 16, emissions of $\mathrm{CO}_{2} \mathrm{e}$ on freeways are expected to decrease by $3 \%$ for a LOS change from $\mathrm{F}$ to $\mathrm{E}$ (average speeds from 19 to $31 \mathrm{mph}$ ), increase by $3 \%$ with a LOS change from E to D (average speeds from 31 to $53 \mathrm{mph}$ ), and decrease by $1 \%$ with a LOS change from D to A-C (average speeds from 53 to $60 \mathrm{mph}$ ).

Table 16. Summary of Percent Change in Emissions on Freeways from CBS (MOVES-Modeled Emissions with $\varepsilon_{q}^{\bar{v}}=0.3$ )

\begin{tabular}{|l|c|c|c|}
\hline Freeway & $\mathbf{1 9 - 3 1} \mathbf{~ m p h}$ & $\mathbf{3 1 - 5 3} \mathbf{~ m p h}$ & $\mathbf{5 3 - 6 0} \mathbf{~ m h}$ \\
\hline $\mathrm{CO}_{2} \mathrm{e}$ & $-3 \%$ & $3 \%$ & $-1 \%$ \\
\hline $\mathrm{CO}$ & $-1 \%$ & $1 \%$ & $0 \%$ \\
\hline $\mathrm{PM}_{2.5}$ & $-3 \%$ & $-12 \%$ & $-6 \%$ \\
\hline $\mathrm{NO}_{\mathrm{x}}$ & $-1 \%$ & $6 \%$ & $0 \%$ \\
\hline $\mathrm{HC}$ & $-13 \%$ & $-9 \%$ & $-3 \%$ \\
\hline
\end{tabular}


Table 17. Summary of Percent Change in Emissions on Arterials from CBS (MOVES-Modeled Emissions with $\varepsilon_{q}^{\bar{v}}=0.3$ )

\begin{tabular}{|l|c|c|c|}
\hline Freeway & $\mathbf{1 0}-\mathbf{1 6} \mathbf{~ m p h}$ & $\mathbf{1 6}-\mathbf{2 4} \mathbf{~ m p h}$ & $\mathbf{2 4}-\mathbf{3 5} \mathbf{~ m p h}$ \\
\hline $\mathrm{CO}_{2} \mathrm{e}$ & $-15 \%$ & $-7 \%$ & $1 \%$ \\
\hline $\mathrm{CO}$ & $-11 \%$ & $-6 \%$ & $-2 \%$ \\
\hline $\mathrm{PM}_{2.5}$ & $-12 \%$ & $-6 \%$ & $-2 \%$ \\
\hline $\mathrm{NO}_{\mathrm{x}}$ & $-11 \%$ & $-5 \%$ & $2 \%$ \\
\hline $\mathrm{HC}$ & $-21 \%$ & $-13 \%$ & $-6 \%$ \\
\hline
\end{tabular}

For EV penetration of the LD fleet the elasticity is slightly more complicated.

Assuming that $e_{e}=0$ and all EV are replacing ICE LD vehicles, then $\frac{\partial f_{l}}{\partial f_{e}}=-1$ and $\frac{\partial f_{h}}{\partial f_{e}}=\frac{\partial q}{\partial f_{e}}=0$. So the elasticity of total emissions $E$ to EV penetration $f_{e}$ is calculated

$$
\varepsilon_{E}^{f_{e}}=\frac{1}{E} \frac{\partial E}{\partial f_{e}}=\frac{1}{q\left[f_{l} e_{l}+f_{h} e_{h}\right]} \frac{\partial}{\partial f_{e}} q\left[f_{l} e_{l}+f_{h} e_{h}\right]=\frac{-e_{l}}{f_{l} e_{l}+f_{h} e_{h}}=\frac{-1}{1+f_{h}\left(e_{h} / e_{l}-1\right)-f_{e}}
$$

For the observed range of $e_{h} / e_{l}$ from around 1 for $\mathrm{CO}$ up to 60 for $\mathrm{PM}_{2.5}$ (see Figure 20) and $f_{h}=0.1, \varepsilon_{E}^{f_{e}}$ can range from $-1.0 \leq \varepsilon_{E}^{f_{e}} \leq-0.1$. Considering LCA EV emissions, the elasticity would be even smaller. Thus, total emissions elasticity of an EV-based NCBS is equal or smaller than the total emissions elasticity to other NCBS. From the denominator of Equation 54 we see that fleets with more HD vehicles $\left(f_{h}\right)$ or pollutants with higher relative emissions rates from $\mathrm{HD}$ vehicles $\left(e_{h} / e_{l}\right)$ will have lower total emissions elasticity to $\mathrm{EV}$ penetration, $\varepsilon_{E}^{f_{e}}$. The latter is the case for $\mathrm{PM}_{2.5}$ emissions in Table 14 and Table 15, where large EV penetrations of the fleet are necessary to produce equivalent emissions reductions to CBS. The relationships in Equation 54 are illustrated 
in Figure 30, where $\varepsilon_{E}^{f_{e}}$ is plotted against $e_{h} / e_{l}$ for $f_{H}=0.1,0.2$, and 0.4 and assuming $f_{e}=0$. The emissions elasticity to $f_{e}$ is reduced with increasing $e_{h} / e_{l}$ and with $f_{H}$.

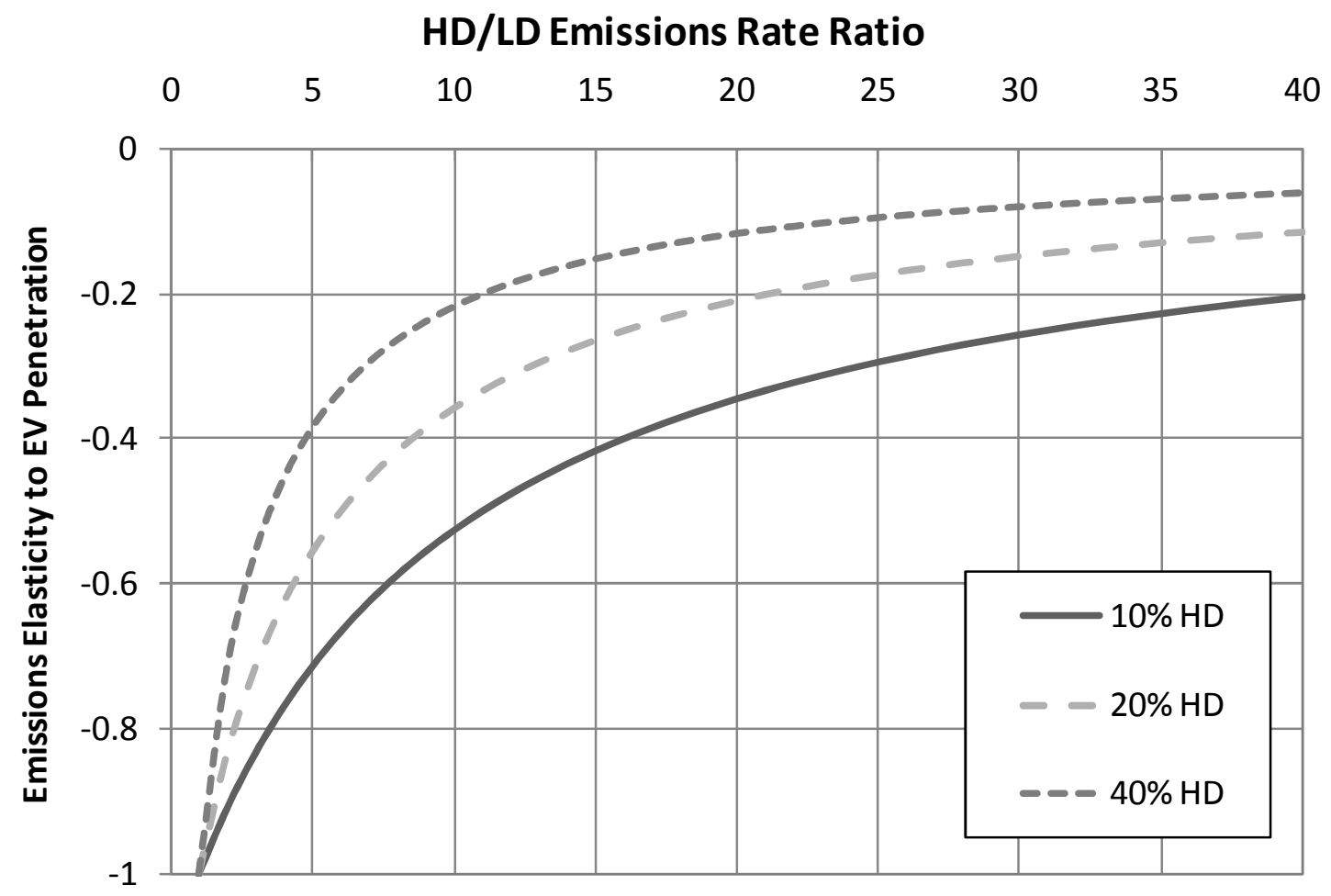

Figure 30. Emissions Elasticity to EV Penetration, $\varepsilon_{E}^{f_{e}}$, versus $e_{h} / e_{l}$, assuming

$$
f_{e}=\mathbf{0} \text { and } e_{e}=0
$$

Looking back at Figure 14 we see that a total emissions elasticity of -1.0 is probably more than can be expected from any speed-based approach (especially if considering induced demand). Thus the emissions elasticity to non-EV NCBS is advantageous. For EV strategies the emissions elasticity is more in line with $\varepsilon_{E}^{\bar{v}}$, though it can vary by an order of magnitude for the observed range of $e_{h} / e_{l}$. When $\varepsilon_{E}^{f_{e}}$ is smaller (less negative) than -1.0 , proportionally more EV penetration is needed to obtain the 
equivalent emissions reductions shown in Table 16 and Table 17, as can be observed in the results above.

In conclusion, there are many conditions (moderate speeds or moderate demand elasticity, for example) in which capacity-based congestion mitigation is likely to increase total emissions. For this reason, emissions reductions cannot be an assumed cobenefit of congestion mitigation. Furthermore, strategies which target emissions reductions directly are more likely to achieve real emissions benefits. But the high emissions rates and high emissions rate sensitivity to speed for HD vehicles raises the potential for emissions benefits from more focused congestion mitigation strategies that target HD vehicles directly. This opportunity is addressed in the next chapter. 


\section{VEHICLE CLASS-SEGREGATED FACILITIES}

LD/HD vehicle differences can have large impacts on total emissions effects of congestion mitigation, as demonstrated above. In addition to having higher emissions rates than $\mathrm{LD}$ vehicles, $\mathrm{HD}$ vehicles are more sensitive to inefficiencies in congestion (Figure 24 and Figure 25). The large contribution to total emission from a small number of $\mathrm{HD}$ vehicles makes them likely targets for more focused emissions and congestion mitigation strategies. For $\mathrm{PM}_{2.5}$, the high values of $\gamma_{q_{h}}^{v_{h}}$ (Figure 25) coupled with HD vehicle dominance of total emissions (Figure 26) suggest potentials for air quality benefits from HD vehicle-specific congestion mitigation strategies. Considering that $\mathrm{PM}_{2.5}$ is a local pollutant with large health risks (Hall et al., 2008; Health Effects Institute, 2010), this could be a particularly important strategy for freight congestion in urban areas.

As a comparison of congestion and emissions mitigation approaches and their class-specific effects, Table 18 shows a short list of emissions mitigation strategies and their expected direct impacts on the key variables of this analysis: traffic speed $v_{j}$, traffic flow volume $q_{j}$, emissions rate parameters $a_{i, j}$, and demand elasticity to speed $\eta_{q_{j}}^{v_{j}}$. The cells in the table are filled in with the relationships of an increase "+", decrease "-", or no change "o". These relationships are of course highly generalized, and actual impacts can depend on details of implementation. Truck-only lanes (TOL) are roadway facilities which provide exclusive right-of-way for HD vehicles (Transportation Research Board, 2010). Just as general capacity expansions can employ road pricing to mitigate induced demand, TOL can utilize lane pricing for the same purpose. 
Table 18. Congestion \& Emissions Mitigation Strategies: Direct Impacts

\begin{tabular}{|c|c|c|c|c|c|c|c|c|}
\hline & \multicolumn{4}{|c|}{ Light-Duty Vehicles } & \multicolumn{4}{|c|}{ Heavy-Duty Vehicles } \\
\hline Strategy & $v_{l}$ & $q_{l}$ & $a_{i, l}$ & $\eta_{q_{l}}^{v_{l}}$ & $v_{h}$ & $q_{h}$ & $a_{i, h}$ & $\eta_{q_{h}}^{v_{h}}$ \\
\hline Capacity increase & + & + & $\mathrm{O}$ & $\mathrm{O}$ & + & + & $\mathrm{O}$ & $\mathrm{O}$ \\
\hline $\begin{array}{l}\text { Truck-only lanes (no toll) - } \\
\text { new capacity }\end{array}$ & + & + & $\mathrm{O}$ & $\mathrm{O}$ & + & + & $\mathrm{O}$ & $\mathrm{O}$ \\
\hline $\begin{array}{c}\text { Truck-only lanes (no toll) - } \\
\text { appropriated capacity }\end{array}$ & - & - & $\mathrm{O}$ & $\mathrm{O}$ & + & + & $\mathrm{O}$ & o \\
\hline $\begin{array}{l}\text { Truck-only lanes (tolled) - } \\
\text { new capacity }\end{array}$ & + & + & $\mathrm{O}$ & $\mathrm{O}$ & + & $\mathrm{o}$ & $\mathrm{O}$ & - \\
\hline $\begin{array}{l}\text { Truck-only lanes (tolled) - } \\
\text { appropriated capacity }\end{array}$ & - & - & O & $\mathrm{O}$ & + & o & o & - \\
\hline $\begin{array}{l}\text { Congestion pricing/demand } \\
\text { reduction strategies }\end{array}$ & + & - & $\mathrm{O}$ & - & + & - & $\mathrm{O}$ & - \\
\hline $\begin{array}{l}\text { Vehicle/fuel efficiency } \\
\text { improvements }\end{array}$ & $\mathrm{O}$ & $\mathrm{o}^{1}$ & - & $\mathrm{O}$ & $\mathrm{o}$ & $\mathrm{o}^{1}$ & - & $\mathrm{O}$ \\
\hline
\end{tabular}

${ }^{1}$ Assuming fuel cost savings do not lead to induced travel

Capacity expansions increase $v_{j}$ and $q_{j}$, and the total emissions effect depends on the relative magnitude of each, as illustrated in Chapter 8. The impact of TOL on LD vehicles depends on whether (a) the TOL are added capacity (in which case $v_{l}$ and $q_{l}$ would increase with the relocation of HD vehicles), or (b) the TOL are appropriated general purpose (GP) capacity (in which case the capacity decrease for LD vehicles would likely lower $v_{l}$ and $q_{l}$, though traffic flow impacts of this type of TOL vary (Middleton, 2006; Transportation Research Board, 2010)). A tolled TOL can have similar efficiency benefits without an increase in truck volumes by offsetting the travel time savings with toll costs (reducing the effective demand elasticity to travel speed). For tolled TOL facilities $\eta_{q_{h}}^{v_{h}}$ decreases because travel speed increases induce less demand.

Congestion pricing and other forms of travel demand management (TDM) similarly reduce demand elasticity to travel speed, $\eta_{q_{j}}^{v_{j}}$, by replacing time savings with 
tolling costs or other motivators. More to the point, TDM aim to reduce traffic volumes and so increase traffic speeds and vehicle efficiency. Non-traffic approaches to emissions reductions include improvements in vehicle and fuel efficiency as analyzed in Section 8.3. Such strategies reduce emissions rates, with the only likely impact on speed or volume being possible induced demand through decreased travel (fuel) costs.

\subsection{Truck-Only Lane Analysis}

In this section we illustrate the expected emissions impacts of several different lane management strategies, including TOL. The base conditions are given as a 3-lane congested freeway facility of arbitrary length (all GP lanes) with the following characteristics and assumptions:

1. $10 \%$ HD vehicles $\left(f_{h}=0.1\right)$ with $P C E_{h}=1.5$ for level terrain (Transportation Research Board, 2000)

2. Demand elasticity to speed of 0.3 for both HD and LD vehicles $\left(\eta_{q_{l}}^{v_{l}}=\eta_{q_{h}}^{v_{h}}=\right.$ 0.3

3. BPR equation parameters as above: $\alpha=0.15, \beta=7, t_{0}=1 \mathrm{~min} / \mathrm{mi}$ (freeflow speed of $60 \mathrm{mph}$ ), and $c=2,200 \mathrm{pcphpl}$

4. Initial volume of $q=2,300$ vphpl (about $10 \%$ over capacity, considering PCE)

5. An even distribution of traffic among all travel lanes

6. On mixed LD/HD facilities, $\mathrm{LD}$ and $\mathrm{HD}$ vehicles travel at the same average speed 
7. When TOL exist, they are mandatory and exclusive for all HD vehicles meaning there are no mixed LD/HD flow lanes when TOL exist.

Four different lane management scenarios are considered, all without tolling:

1. Convert one of the GP lanes to a TOL

2. Add a TOL

3. Add a GP lane

4. Remove a GP lane

For these calculations arc demand elasticities are used (similar to Equation 12), meaning that if the initial demand volume and speed for vehicles of class $j$ are $q_{j_{1}}$ and $v_{j_{1}}$,

respectively, we can estimate the new demand volume $q_{j_{2}}$ from the new speed, $v_{j_{2}}$, using

$$
\begin{aligned}
& \eta_{q_{j}}^{v_{j}}=\frac{\left(v_{j_{2}}+v_{j_{1}}\right)\left(q_{j_{2}}-q_{j_{1}}\right)}{\left(q_{j_{2}}+q_{j_{1}}\right)\left(v_{j_{2}}-v_{j_{1}}\right)} \\
& q_{j_{2}}=q_{j_{1}} \frac{v_{j_{1}}+v_{j_{2}}+\eta_{q_{j}}^{v_{j}}\left(v_{j_{2}}-v_{j_{1}}\right)}{v_{j_{1}}+v_{j_{2}}-\eta_{q_{j}}^{v_{j}}\left(v_{j_{2}}-v_{j_{1}}\right)}
\end{aligned}
$$

But since the additional volume, $q_{j_{2}}-q_{j_{1}}$, impacts the speed, $v_{j_{2}}$, we must also consider the relationship between $v_{j_{2}}$ and $q_{j_{2}}$. If $q_{2}^{\prime}$ is the final volume in PCE, then using the BPR function (Equation 1) $v_{j_{2}}=\frac{1}{t_{o}\left(1+\alpha\left(q^{\prime}{ }_{2} / c\right)^{\beta}\right)}$. Rearranging Equation 55 and substituting for $v_{j_{2}}$ based on the BPR function we get

$$
q_{j_{2}}\left[\frac{v_{j_{1}}}{v_{j_{2}}}+1-\eta_{q_{j}}^{v_{j}}+\eta_{q_{j}}^{v_{j}} \frac{v_{j_{1}}}{v_{j_{2}}}\right]=q_{j_{1}}\left[\frac{v_{j_{1}}}{v_{j_{2}}}+1+\eta_{q_{j}}^{v_{j}}-\eta_{q_{j}}^{v_{j}} \frac{v_{j_{1}}}{v_{j_{2}}}\right]
$$




$$
\begin{gathered}
q_{j_{2}}\left[q^{\prime \beta} v_{j_{1}} t_{o} \alpha c^{-\beta}\left(1+\eta_{q_{j}}^{v_{j}}\right)+v_{j_{1}} t_{o}\left(1+\eta_{q_{j}}^{v_{j}}\right)+1-\eta_{q_{j}}^{v_{j}}\right]+q^{\prime \beta} q_{j_{1}} v_{j_{1}} t_{o} \alpha c^{-\beta}(1- \\
\eta q j v j=q j 1 v j 1 \text { to } 1-\eta q j v j+q j 11+\eta q j v j .
\end{gathered}
$$

Then we can calculate

$$
q_{2}^{\prime}=\sum_{j \in J} q_{j_{2}} \cdot P C E_{j}
$$

For two (LD and HD) vehicle classes, $J=\{l, h\}$, Equations 56 and 57 represent a system of three equations with three unknowns: $q_{l_{2}}, q_{h_{2}}$, and $q_{2}^{\prime}$. All other variables are parameters or initial conditions. Therefore the equations can be simultaneously solved to find the final volumes and speeds for each vehicle class. The final volume and speed for each vehicle class satisfy both the demand elasticity $\eta_{q_{j}}^{v_{j}}$ and the theoretical volume/speed relationship (BPR). As with the previous analyses, we assume that all VMT changes from variable demand are reflected in changing $q$.

The results of this analysis for total $\mathrm{CO}_{2} \mathrm{e}$ emissions are shown in Table 19. This table shows results for base conditions and all four lane strategies, with absolute values and percent changes from base conditions for class-specific volumes, speeds, and emissions. Interestingly, the largest total emissions benefit is for a TOL conversion. Both TOL scenarios reduce HD vehicle emissions, $E_{h}$, by $8 \%$, but the lane conversion also reduces $v_{l}$ enough to suppress $q_{l}$ by $10 \%$ and reduce $E_{l}$ by $6 \%$. A TOL as additional capacity produces no net change in $E_{l}$, with decreased emissions rates $e_{l}$ but a $4 \%$ increase in $q_{l}$. The $8 \%$ increased $q_{h}$ with TOL is not enough to offset the increased efficiency for $\mathrm{HD}$ vehicles. Adding a GP lane has similar $\mathrm{CO}_{2} \mathrm{e}$ emissions benefits to adding a TOL lane. Removing a GP lane has larger emissions benefits than adding either type of lane, due to the $17 \%$ suppressed demand for both LD and HD vehicles. 
Table 19. Volume, Speed, and $\mathrm{CO}_{2} \mathrm{e}$ Emissions Changes with Lane Strategies

\begin{tabular}{|l|r|rr|rr|rr|rr|}
\hline & $\begin{array}{c}\text { Base } \\
\text { Conditions }\end{array}$ & \multicolumn{2}{|c|}{$\begin{array}{c}\text { TOL } \\
\text { Conversion }\end{array}$} & \multicolumn{2}{|c|}{ TOL Added } & \multicolumn{2}{c|}{$\begin{array}{c}\text { GP Lane } \\
\text { Added }\end{array}$} & \multicolumn{2}{c|}{$\begin{array}{c}\text { GP Lane } \\
\text { Removed }\end{array}$} \\
\hline $\boldsymbol{q}_{\boldsymbol{l}}(\mathrm{veh} / \mathrm{hr})$ & 6,210 & 5,606 & $-10 \%$ & 6,460 & $4 \%$ & 6,594 & $6 \%$ & 5,185 & $-17 \%$ \\
\hline $\boldsymbol{q}_{\boldsymbol{h}}(\mathrm{veh} / \mathrm{hr})$ & 690 & 744 & $8 \%$ & 744 & $8 \%$ & 733 & $6 \%$ & 576 & $-17 \%$ \\
\hline $\boldsymbol{v}_{\boldsymbol{l}}(\mathrm{mph})$ & 47 & 33 & $-29 \%$ & 53 & $14 \%$ & 57 & $22 \%$ & 25 & $-46 \%$ \\
\hline $\boldsymbol{v}_{\boldsymbol{h}}(\mathrm{mph})$ & 47 & 60 & $29 \%$ & 60 & $29 \%$ & 57 & $22 \%$ & 25 & $-46 \%$ \\
\hline $\begin{array}{c}\boldsymbol{E}_{\boldsymbol{l}}(\mathrm{kg} \mathrm{CO2e} \\
/ \mathrm{hr} / \mathrm{road}-\mathrm{mile})\end{array}$ & 2,333 & 2,188 & $-6 \%$ & 2,337 & $0 \%$ & 2,332 & $0 \%$ & 2,150 & $-8 \%$ \\
\hline $\begin{array}{c}\boldsymbol{E}_{\boldsymbol{h}}(\mathrm{kg} \text { CO2e } \\
/ \mathrm{hr} / \mathrm{road}-\mathrm{mile})\end{array}$ & 1,044 & 960 & $-8 \%$ & 960 & $-8 \%$ & 980 & $-6 \%$ & 1015 & $-3 \%$ \\
\hline $\begin{array}{c}\boldsymbol{E}(\mathrm{kg} \text { CO2e } \\
/ \mathrm{hr} / \text { road-mile })\end{array}$ & 3,377 & 3,148 & $-7 \%$ & 3,297 & $-2 \%$ & 3,312 & $-2 \%$ & 3,165 & $-6 \%$ \\
\hline
\end{tabular}

Figure 31 shows the results of this analysis for all five pollutants as the percent change in total emissions from base conditions for each strategy. Of the TOL strategies, GP lane conversion outperforms lane addition from an emissions perspective for all pollutants except HC. Adding a TOL produces lower total emissions than adding a GP lane for all pollutants. GP lane removal has mixed effects. $\mathrm{PM}_{2.5}$ and $\mathrm{HC}$ emissions both increase, while $\mathrm{NO}_{\mathrm{x}}$ shows its greatest decrease of all the strategies. These results are intuitive: $\mathrm{HC}$ and $\mathrm{PM}_{2.5}$ are more sensitive to speed than $\mathrm{NO}_{\mathrm{x}}$ (see Figure 4), so more likely to benefit from a speed increase (adding a lane), while $\mathrm{NO}_{\mathrm{x}}$ is more likely to benefit from reduced volume (suppressed demand from removing a lane). 


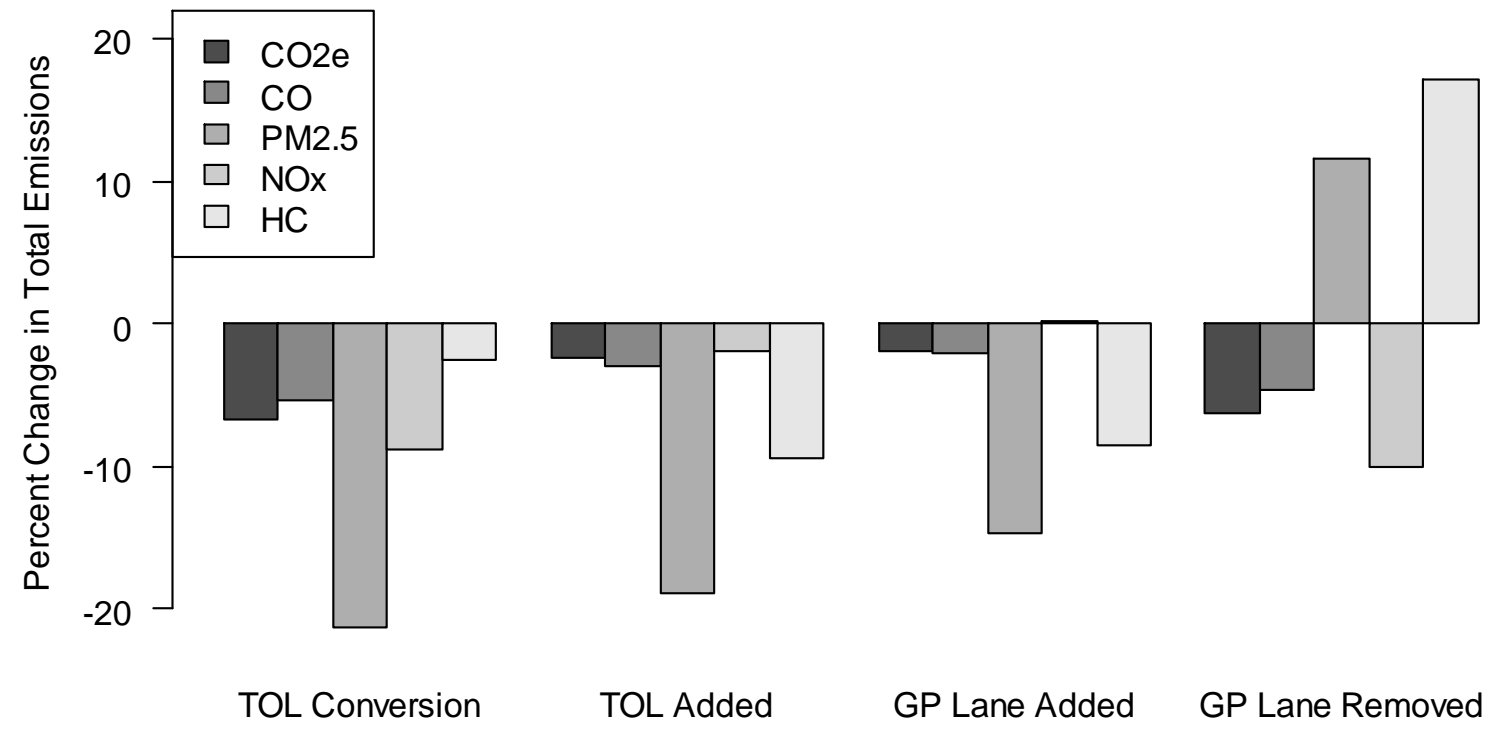

Figure 31. Percent Reductions in Total Emissions for Each Land Strategy and Pollutant

The next four plots explore sensitivity of these results to several key characteristics and assumptions: initial volume, fraction HD vehicles, and demand elasticity to speed. Figure 32 shows the percent change in total $\mathrm{CO}_{2} \mathrm{e}$ emissions for varying initial volumes. For low initial volumes with nearly free-flow conditions the emissions effects are minimal, with the exception of removing a GP lane which increases emissions. At volumes around the initial conditions of 6,900 veh/hour the largest potential benefits are for GP lane reductions (with or without TOL) - though GP lane removal without TOL degrades to an emissions increase around 7,500 veh/hour. Both additional lane scenarios also increase total emissions for higher initial volumes because of induced demand. 


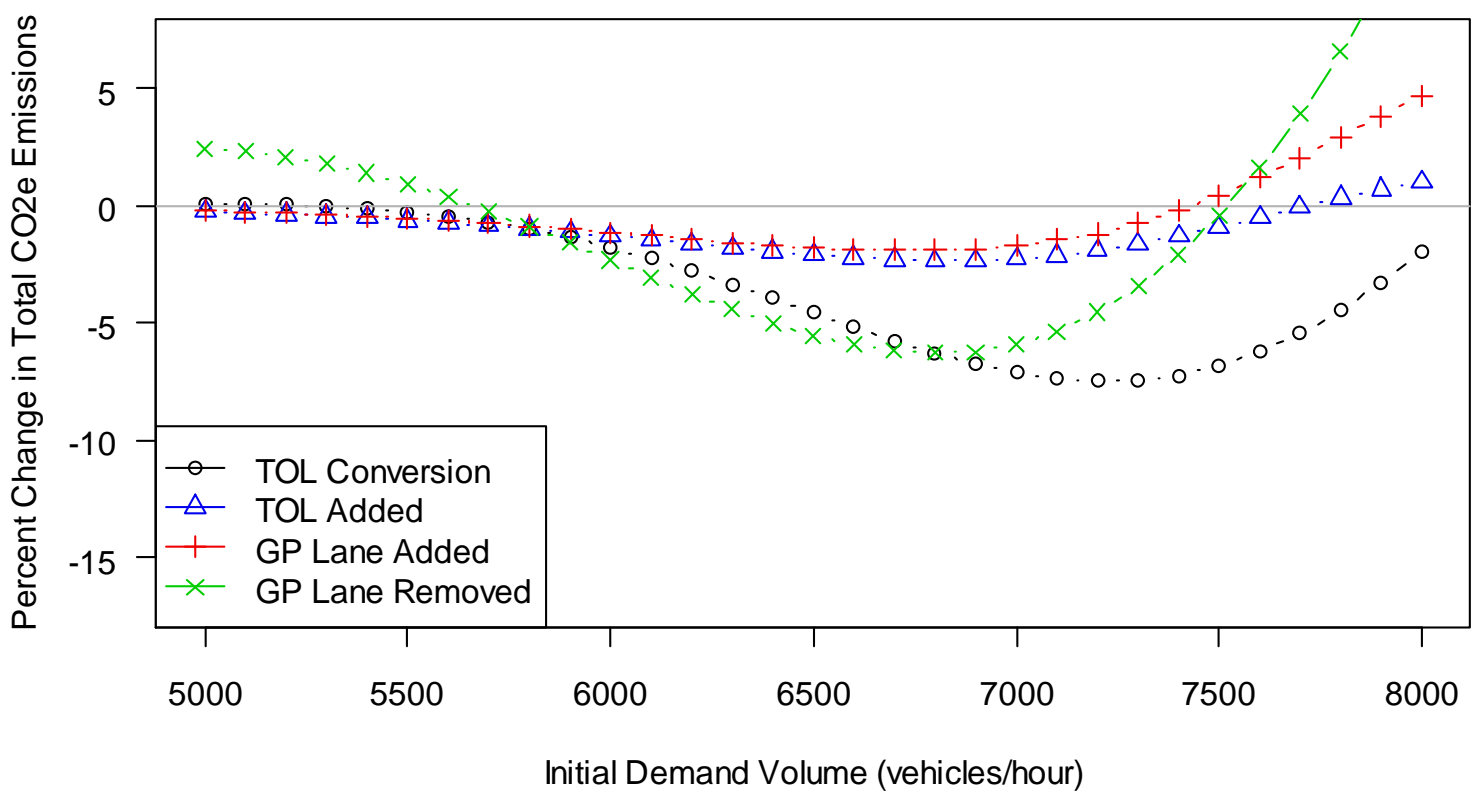

Figure 32. Effect of Lane Strategies on Total $\mathrm{CO}_{2} \mathrm{e}$ Emissions with Varying Initial $q$ (Vehicle Travel Demand for 3 GP Lanes); Other Assumptions as Above

Figure 33 shows the percent change in total $\mathrm{CO}_{2} \mathrm{e}$ emissions with varying initial fractions of HD vehicles in the fleet, $f_{H}$. At high initial $f_{H}$ the TOL strategies are not effective at reducing emissions because the TOL are saturated and not operating at efficient speeds for the HD vehicles. At very low truck volumes additional TOL are minimally utilized and have little effect, while TOL conversion suppresses LD demand enough to reduce total emissions. GP lane addition is fairly insensitive to fraction HD vehicles, while GP lane removal is decreasingly effective at higher fractions of HD vehicles because HD vehicles are proportionally more inefficient at very low speeds than LD vehicles. 


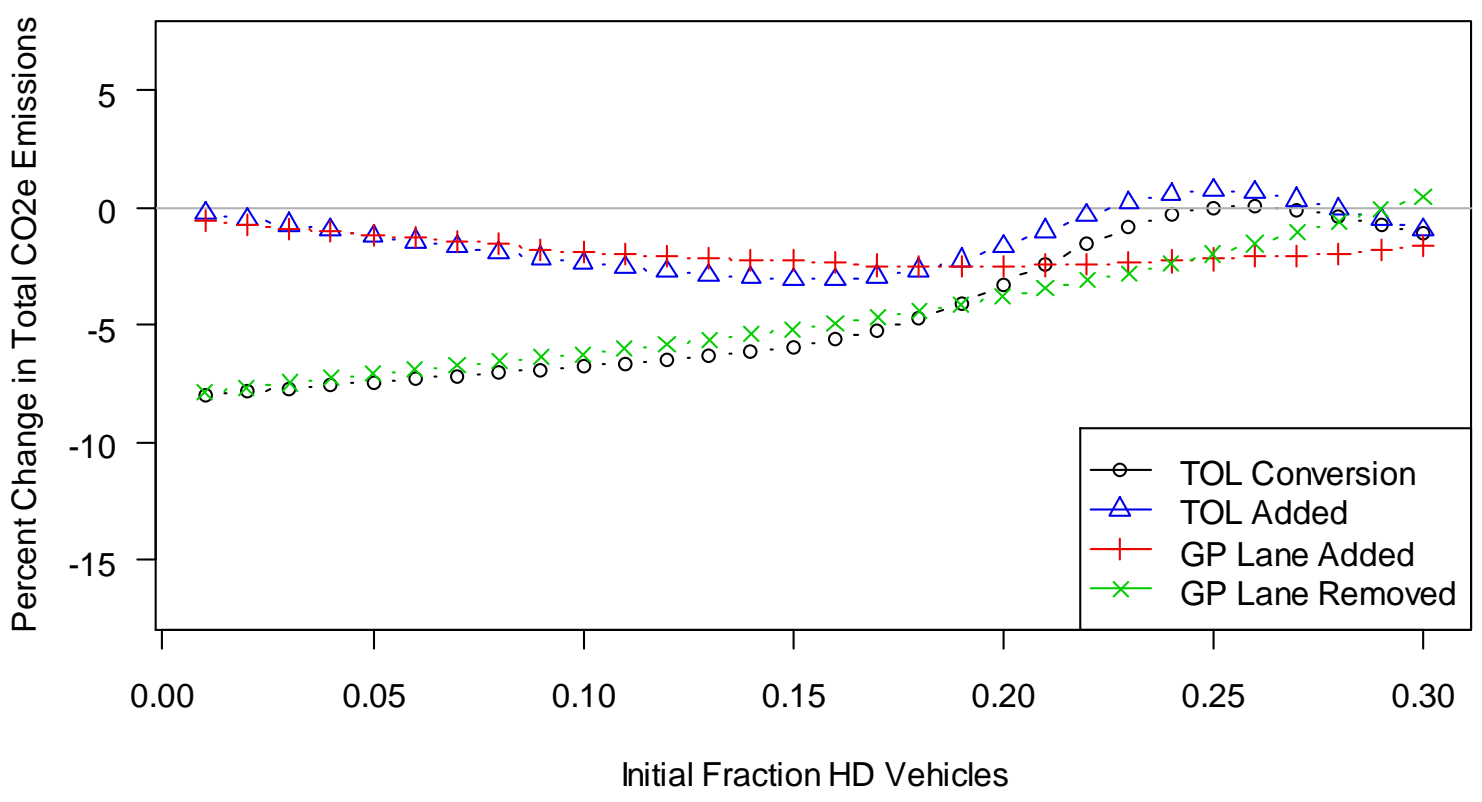

Figure 33. Effect of Lane Strategies on Total $\mathrm{CO}_{2} \mathrm{e}$ Emissions with Varying Initial $f_{H}$; Other Assumptions as Above

Figure 34 shows the effect on total emissions of varying demand elasticity to

speed $\eta_{q_{j}}^{v_{j}}$ (assumed the same for both vehicle classes). Total emissions from lane additions (TOL or GP) increase nearly linearly with increasing demand elasticity as the increased capacity induces an increasing amount of travel. Increasing demand elasticity has the opposite effect on GP capacity reductions as an increasing amount of demand (and emissions) are suppressed at higher elasticities. This effect is offset somewhat at very high elasticities as the TOL conversion induces an excessive amount of HD vehicle travel. At low elasticities the lane reductions are particularly ineffective because they decrease efficiency without suppressing demand. From this figure we see that the assumed elasticity of 0.3 is in a narrow range which leads to total $\mathrm{CO}_{2} \mathrm{e}$ emissions reductions for all four strategies. 


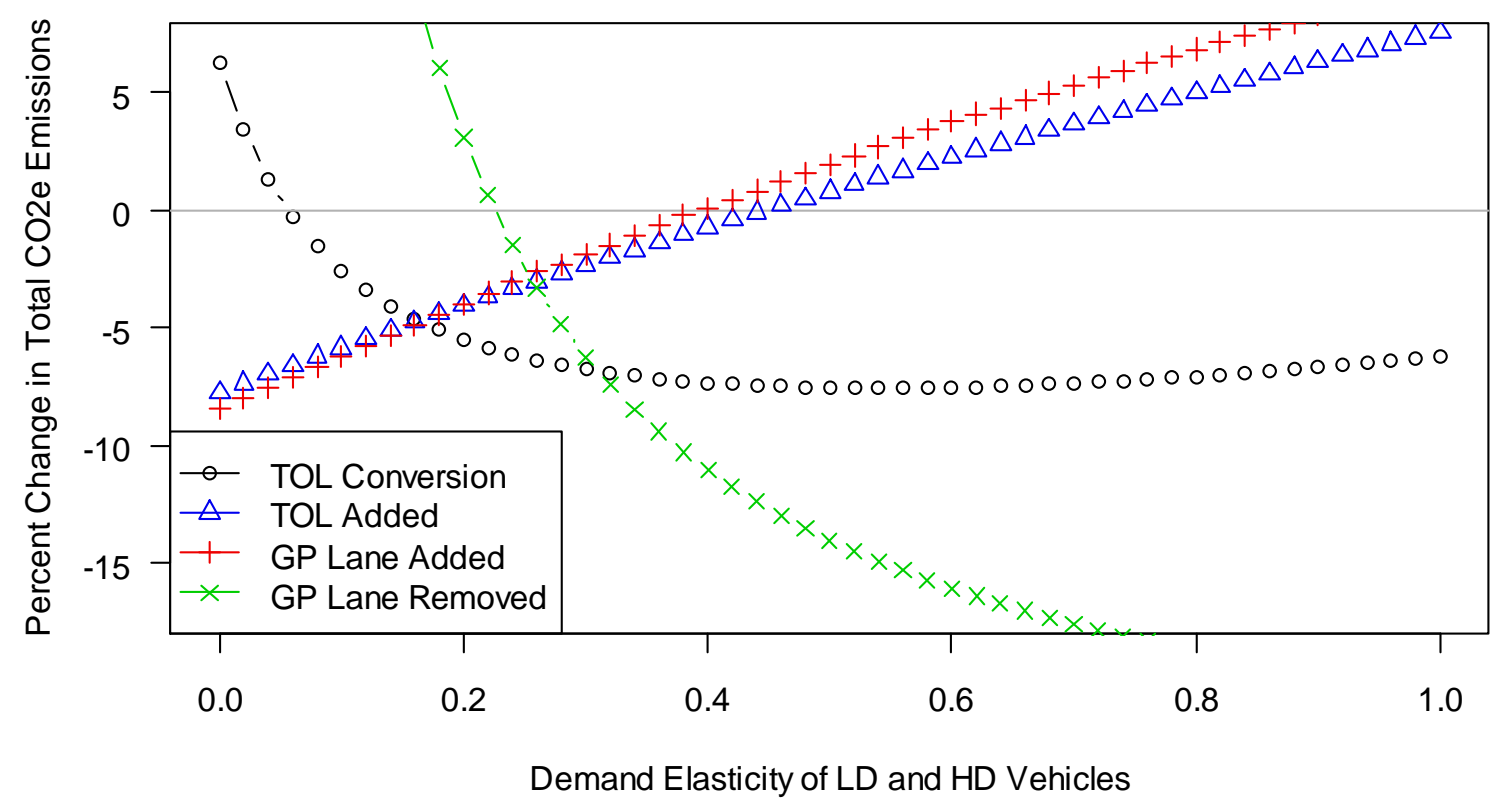

Figure 34. Effect of Lane Strategies on Total $\mathrm{CO}_{2} \mathrm{e}$ Emissions with Varying Demand Elasticity $\boldsymbol{\eta}_{q_{j}}^{v_{j}}$ (for Both LD and HD Vehicles); Other Assumptions as Above

Figure 35 looks at the total emissions results from varying only HD vehicle demand elasticity to speed, $\eta_{q_{h}}^{v_{h}}$. The results are similar to Figure 34 with the marked exception of the emissions impact of TOL conversion at low HD demand elasticity. In Figure 35, TOL conversion is increasingly effective at low demand elasticity because it continues to suppress LD vehicle demand, unlike in Figure 34. The other strategies have similar shapes in Figure 35 as compared to Figure 34, though with less sensitivity. As a final note, although simple GP lane removal outperforms TOL conversion for emissions reductions in some situations (particularly for high $\eta_{q_{h}}^{v_{h}}$ ), TOL conversion is more likely to be a politically feasible option for implementation (particularly if it garners the support of the trucking industry). 


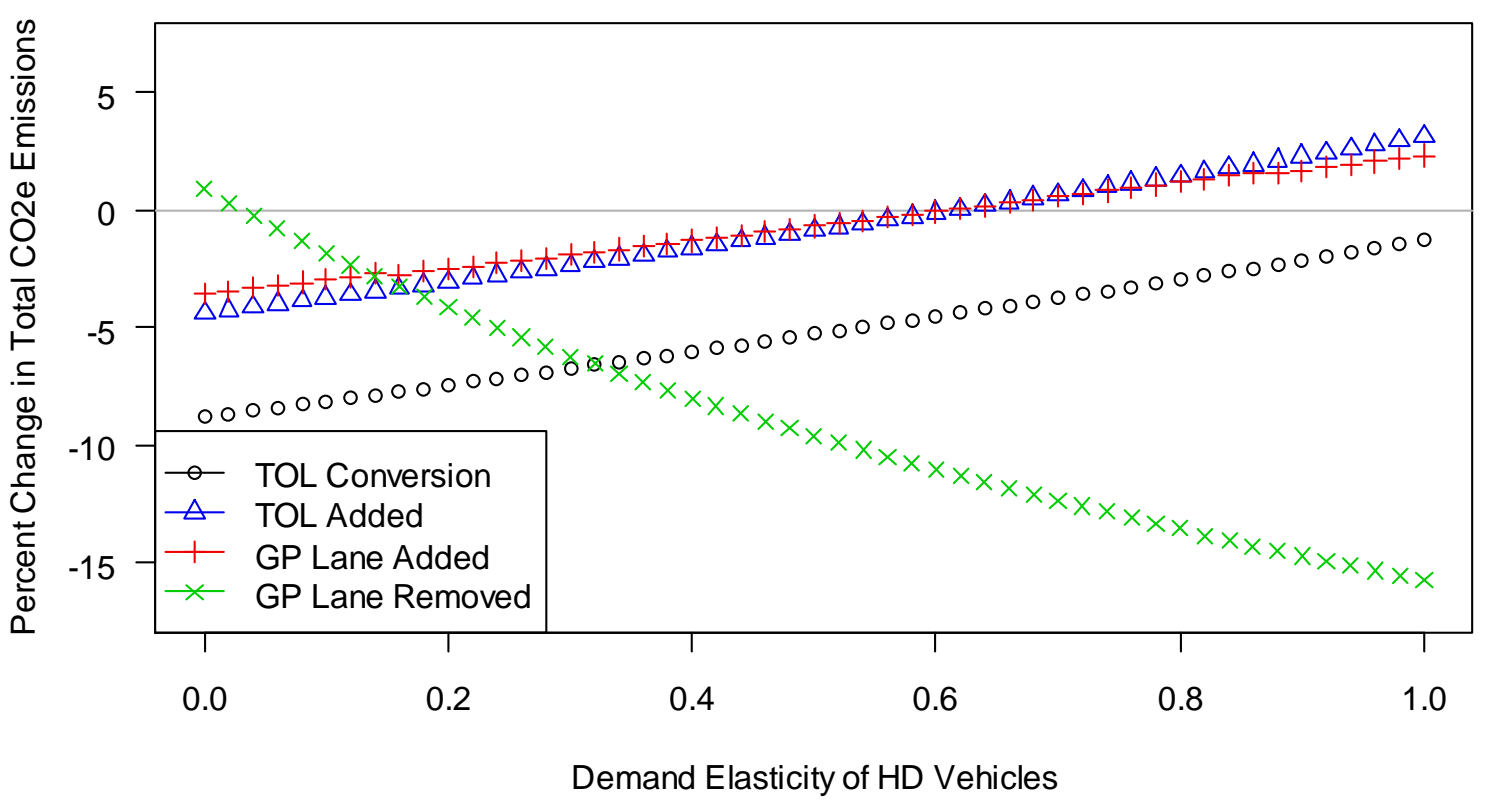

\section{Figure 35. Effect of Lane Strategies on Total $\mathrm{CO}_{2} \mathrm{e}$ Emissions with Varying Demand Elasticity for HD Vehicles; Other Assumptions as Above}

In their analysis of the emissions impacts of tolled TOL, Chu and Meyer (2009) predict net emissions reductions of 3-66\% and $61-62 \%$ for $\mathrm{HC}$ and $\mathrm{CO}_{2}$, respectively, and net emissions increases of $2-5 \%$ and $1-18 \%$ for $\mathrm{CO}$ and $\mathrm{NO}_{\mathrm{x}}$, respectively. Besides different emissions rate curves, their analysis used a travel demand model to estimate volume changes. The details of the demand model and its results for the studied scenarios are not described in the paper. Since speed results are not provided we cannot compare the implied demand elasticity with this analysis. The TOL are added capacity, and they do predict an increase in LD vehicle volumes on the GP lanes accordingly. It is surprising, though, that they predict a decrease in total volumes with the added TOL capacity (this is not explained in the paper). The emissions results are more or less in line with what is expected from this analysis, given the higher fraction of HD vehicles (about $19 \%$ ) - with the exception of $\mathrm{CO}_{2}$ emissions. Their expected benefit of over $61 \%$ for $\mathrm{CO}_{2}$ 
emissions is surprisingly high, particularly given the net volume change of less than $3 \%$. This may be due to the rough approximation of $\mathrm{CO}_{2}$ emissions used in the paper: a simple percent difference in cubed speeds.

In conclusion, truck-specific congestion mitigation strategies could have more potential for emissions reductions than general purpose congestion mitigation. This is particularly true if truck travel demand elasticity to travel time is moderate or low. In fact, converting a general purpose lane to a truck-only lane can have more emissions benefits than adding capacity, despite the increased congestion for light-duty vehicles. In the next chapter of this thesis we return to composite fleet analysis and look at congestion performance measures and their applicability for emissions trends. 


\section{IMPLICATIONS FOR PERFORMANCE MEASURES}

In this final results chapter we look at how the preceding analysis can inform the application of congestion performance measures for emissions considerations.

\subsection{Speed-Based Performance Measures}

Speed-based or delay-based performance measures are common when assessing congestion levels. These mobility-oriented metrics compare a congested speed or travel time with some threshold of uncongested conditions. They generally are normalized to travel distance, estimating the excess travel time per mile or with respect to a trip of a fixed length. Examples include the Travel Time Index (TTI), Buffer Time Index, and the Planning Time Index - see Cambridge Systematics, Inc. (2005). The TTI, in particular, enjoys extensive use in the Texas Transportation Institute's Urban Mobility Report (UMR) (Schrank et al., 2010). The TTI is calculated as the ratio of average peak-period travel time to the travel time on the same facilities in off-peak/free-flow conditions,

$$
T T I=\frac{\bar{t}}{t_{o}}=\frac{v_{o}}{\bar{v}}
$$

where $\bar{t}$ and $\bar{v}$ are the average peak-period travel rate and travel speed, respectively, and $t_{o}$ and $v_{o}$ are the off-peak (free-flow) travel rate and travel speed, respectively.

Since $\bar{v}=\frac{v_{o}}{T T I}, \frac{\partial \bar{v}}{\partial T T I}=\frac{-v_{o}}{\operatorname{TTI}^{2}}=\frac{-\bar{v}^{2}}{v_{o}}$ and total emissions, E, elasticity to the TTI can be calculated

$$
\varepsilon_{E}^{T T I}=\frac{T T I}{E} \frac{\partial E}{\partial T T I}=\frac{v_{o}}{E \bar{v}} \frac{\partial E}{\partial \bar{v}} \frac{\partial \bar{v}}{\partial T T I}=\frac{v_{O}}{E \bar{v}}\left(\varepsilon_{E}^{\bar{v}} \frac{E}{\bar{v}}\right)\left(\frac{\bar{v}^{2}}{v_{o}}\right)=-\varepsilon_{E}^{\bar{v}} .
$$

We can then go back to the $\varepsilon_{E}^{\bar{v}}$ figures in Section 5.5 to see the conditions (for average speed and demand elasticity) where total emissions are expected to increase or decrease 
with the TTI. Similar to the case of congestion mitigation, we see there are many situations (particularly for moderate congestion levels and demand elasticities) where total emissions will decrease with increasing TTI. In other words, emissions are moving in the opposite direction as the congestion performance measure. For this reason speedbased congestion measures such as the TTI should not be viewed as indicators of poor performance from an emissions perspective.

Total emissions do not track with speed/delay metrics partly because speed-based performance measures fail to account for volume changes - which are important from an emissions perspective. Only considering the direct impacts of speed changes on emissions rates (without volume changes) the total emissions elasticity to the TTI would be the same as the emissions rate elasticity to the TTI:

$$
\begin{gathered}
\left(\varepsilon_{E}^{T T I}\right)_{\frac{\partial q}{\partial \bar{v}}=0}=\frac{T T I}{E} q \frac{\partial \bar{e}}{\partial T T I}=\frac{T T I}{\bar{e}} \frac{\partial \bar{e}}{\partial T T I}=\varepsilon_{\bar{e}}^{T T I} \\
\varepsilon_{\bar{e}}^{T T I}=\frac{v_{o}}{\bar{e} \bar{v}} \frac{\partial \bar{e}}{\partial \bar{v}} \frac{\partial \bar{v}}{\partial T T I}=\frac{v_{o}}{E \bar{v}}\left(\varepsilon_{\bar{e}}^{\bar{v}} \frac{\bar{e}}{\bar{v}}\right)\left(\frac{-\bar{v}^{2}}{v_{o}}\right)=-\varepsilon_{\bar{e}}^{\bar{v}} .
\end{gathered}
$$

It then follows that

$$
\varepsilon_{E}^{T T I}-\varepsilon_{\bar{e}}^{T T I}=-\varepsilon_{E}^{\bar{v}}+\varepsilon_{\bar{e}}^{\bar{v}}=-\left(\eta_{q}^{\bar{v}}+\varepsilon_{\bar{e}}^{\bar{v}}\right)+\varepsilon_{\bar{e}}^{\bar{v}}=-\eta_{q}^{\bar{v}}
$$

Since $\varepsilon_{\bar{e}}^{\bar{v}}$ is mostly in the range of 0.0 to -1.0 (see Figure 13) and $\eta_{q}^{\bar{v}}$ is expected to be between 0.0 and 1.0, the impact of using a volume-insensitive performance measure such as the TTI to indicate emissions performance is potentially large (the effect of neglecting volume changes is on the same order as the effect of the speed change itself). In fact, neglecting demand can potentially change the direction of the relationship between emissions and the performance measure (i.e. expecting a positive instead of negative 
emissions elasticity to the TTI). Any performance measure that only considers speed, not travel quantity (volume or distance), will likely misrepresent the total emissions relationship with congestion.

\subsection{Performance Measure Examples}

As an example we can look at the performance measures implied by the lane strategies described and analyzed in Section 9.1. The TTI can be calculated from the speeds in Table 19, leading to the values shown in Table 20 (assuming $v_{o}=60 \mathrm{mph}$ ), where $T T I_{l}$ and $T T I_{h}$ are the TTI values computed using $v_{l}$ and $v_{h}$, respectively. Comparing the percent changes in total emissions and TTI for each vehicle class and for the total roadway, we see that the TTI is a poor predictor of emissions impacts. For GP lane removals (with and without TOL) the TTI moves in the opposite direction as total emissions (because the TTI does not account for the suppressed demand volume). Percent volume changes are also shown in Table 20, and although there are still large discrepancies between volume changes and total emissions changes (particularly for HD vehicles), the percent differences for $q$ and $q_{j}$ are closer to the percent differences for $E$ and $E_{j}$ than are the percent differences in the TTI. 
Table 20. TTI and Total Emissions Effects of Lane Strategies Described in Section

9.1

\begin{tabular}{|c|c|c|c|c|c|c|c|c|c|}
\hline & $\begin{array}{c}\text { Base } \\
\text { Conditions }\end{array}$ & $\begin{array}{r}\mathrm{T} \\
\text { Conv }\end{array}$ & ion & TOLA & dded & $\begin{array}{l}\text { GP L } \\
\text { Add }\end{array}$ & & $\begin{array}{l}\text { GP } \\
\text { Rem }\end{array}$ & $\begin{array}{l}\text { ane } \\
\text { ved }\end{array}$ \\
\hline $\begin{array}{l}\boldsymbol{E}_{\boldsymbol{l}} \text { (kg CO2e } \\
/ \mathrm{hr} / \text { road-mile) }\end{array}$ & 2,333 & 2,188 & $-6 \%$ & 2,337 & $0 \%$ & 2,332 & $0 \%$ & 2,150 & $-8 \%$ \\
\hline $\boldsymbol{q}_{l}(\mathrm{veh} / \mathrm{hr})$ & 6,210 & 5,606 & $-10 \%$ & 6,460 & $4 \%$ & 6,594 & $6 \%$ & 5,185 & $-17 \%$ \\
\hline$T T I_{l}$ & 1.28 & 1.82 & $42 \%$ & 1.13 & $-12 \%$ & 1.05 & $-18 \%$ & 2.40 & $88 \%$ \\
\hline $\begin{array}{l}\boldsymbol{E}_{\boldsymbol{h}}(\mathrm{kg} \mathrm{CO} 2 \mathrm{e} \\
/ \mathrm{hr} / \text { road-mile })\end{array}$ & 1,044 & 960 & $-8 \%$ & 960 & $-8 \%$ & 980 & $-6 \%$ & 1015 & $-3 \%$ \\
\hline $\boldsymbol{q}_{\boldsymbol{h}}(\mathrm{veh} / \mathrm{hr})$ & 690 & 744 & $8 \%$ & 744 & $8 \%$ & 733 & $6 \%$ & 576 & $-17 \%$ \\
\hline$T T I_{h}$ & 1.28 & 1.00 & $-22 \%$ & 1.00 & $-22 \%$ & 1.05 & $-18 \%$ & 2.40 & $88 \%$ \\
\hline $\begin{array}{l}\boldsymbol{E} \text { (kg CO2e } \\
/ \mathrm{hr} / \text { road-mile) }\end{array}$ & 3,377 & 3,148 & $-7 \%$ & 3,297 & $-2 \%$ & 3,312 & $-2 \%$ & 3,165 & $-6 \%$ \\
\hline $\boldsymbol{q}$ (veh/hr) & 6,900 & 6,350 & $-8 \%$ & 7,204 & $4 \%$ & 7,327 & $6 \%$ & 5,761 & $-17 \%$ \\
\hline TTI & 1.28 & 1.66 & $30 \%$ & 1.12 & $-13 \%$ & 1.05 & $-18 \%$ & 2.40 & $88 \%$ \\
\hline
\end{tabular}

As another, more macroscopic example we can compare the approximate peak period emissions and congestion performance from all the urban areas described in the UMR (Schrank et al., 2010). The UMR data tables provide estimates of the TTI for each of 101 U.S. Urban Areas. From Equation 58 we can estimate $\bar{v}$ for each urban area on freeway and arterial facilities, assuming the TTI is the same on each and assuming freeflow speeds of 60 and $35 \mathrm{mph}$, respectively (from the UMR methodology). We can then calculate $\bar{e}(\bar{v})$ for each facility and urban area combination using the ESC fit parameters from Table 4 and Table 5. Next we estimate total peak period emissions for each urban area as the summed product of $\bar{e}$ and total peak period VMT on each facility (peak-period VMT is estimated as half of the facility's daily VMT, as per the UMR methodology). Finally, daily peak-period emissions per peak-period traveler are calculated for each urban area using the number of peak period travelers in the UMR data tables. This is a 
highly aggregate approach, but useful for loose comparisons across many cities. Note that the emissions rates versus average speed curves are assumed to be as the same across cities.

Estimated daily peak period $\mathrm{CO}_{2} \mathrm{e}$ emissions per peak period traveler are shown in Figure 36 for 2009, with Urban Areas indicated by population category (Small: $<1 / 2$ million, Medium: 1/2 - 1 million, Large: 1-3 million, and VeryLarge: > 3 million population). Figure 37, Figure 38, and Figure 39 also compare all 101 urban areas in the UMR for 2009, segmented by population category, for different emissions and congestion variables. Comparing amongst urban areas Figure 36, total emissions per traveler and TTI have essentially no relationship. Although emissions rates $(\bar{e}$, per vehicle-mile) increase somewhat with the TTI, the average travel distances do not - and emissions per traveler correlate strongly with average daily peak period VMT per peak period traveler, as shown in Figure 37. We get similar results if we look at total emissions and VMT per capita instead of per peak-period traveler. 


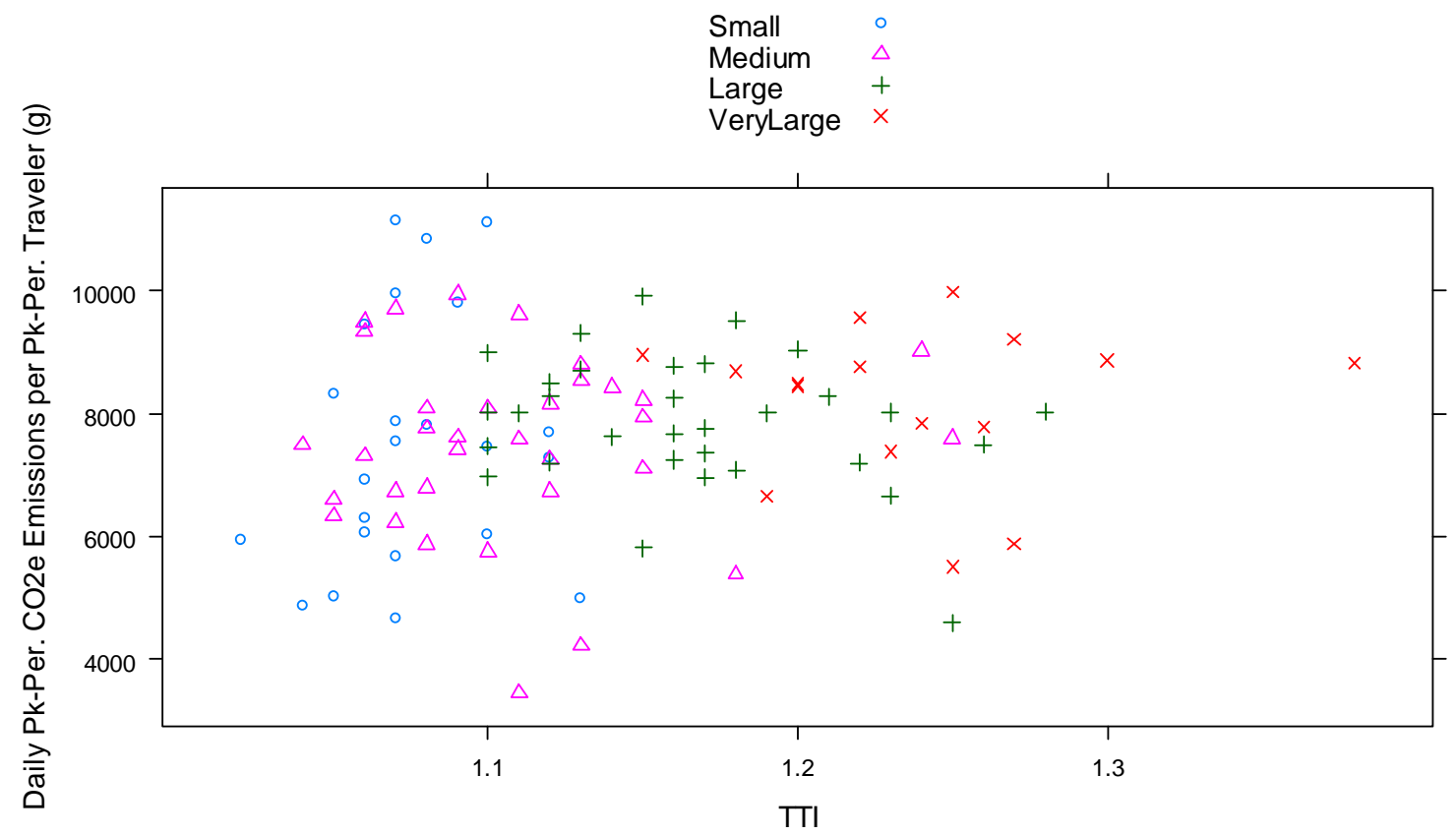

Figure 36. Daily Peak-Period $\mathrm{CO}_{2} \mathrm{e}$ Emissions per Peak-Period Traveler versus TTI, Segmented by Urban Area Population Size

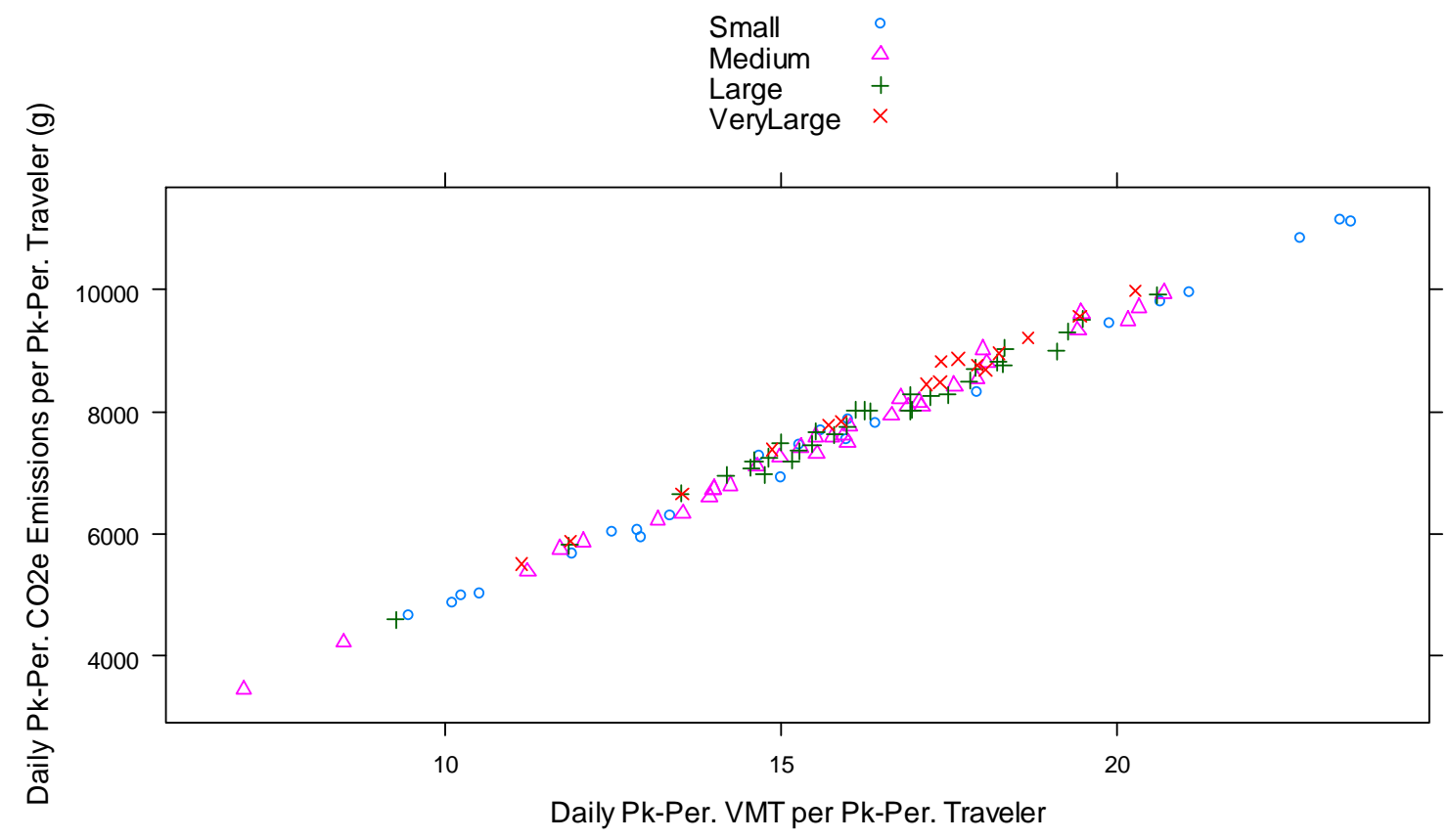

Figure 37. Daily Peak-Period $\mathrm{CO}_{2}$ e Emissions per Peak-Period Traveler versus Peak-Period VMT per Peak-Period Traveler, Segmented by Urban Area Population Size 
Total daily peak period emissions (not per traveler or per capita) do trend upward somewhat with TTI, as shown in Figure 38. This makes sense because the number of peak period travelers and population are both positively correlated with the TTI. But when we stratify by population category (as is done in Figure 38), we see that within population categories the TTI does not correlate with increasing total emissions. The two very high emitting urban areas are New York and Los Angeles, each with populations well above 10 million. As such, they better represent a fifth, "Extremely Large" population group, with high total emissions and TTI. What we see from the categorization in Figure 38 is that high total emissions are associated with larger population areas, not necessarily higher TTI's (although those two are correlated). Comparing total emissions with total VMT reveals - similar to what is shown in Figure 37 - that total emissions are much more strongly correlated with VMT than TTI (not plotted here because it essentially duplicates Figure 37). For these reasons volume measures are preferable to speed measures as emissions performance indicators, although ideally emissions performance metrics incorporate both. 


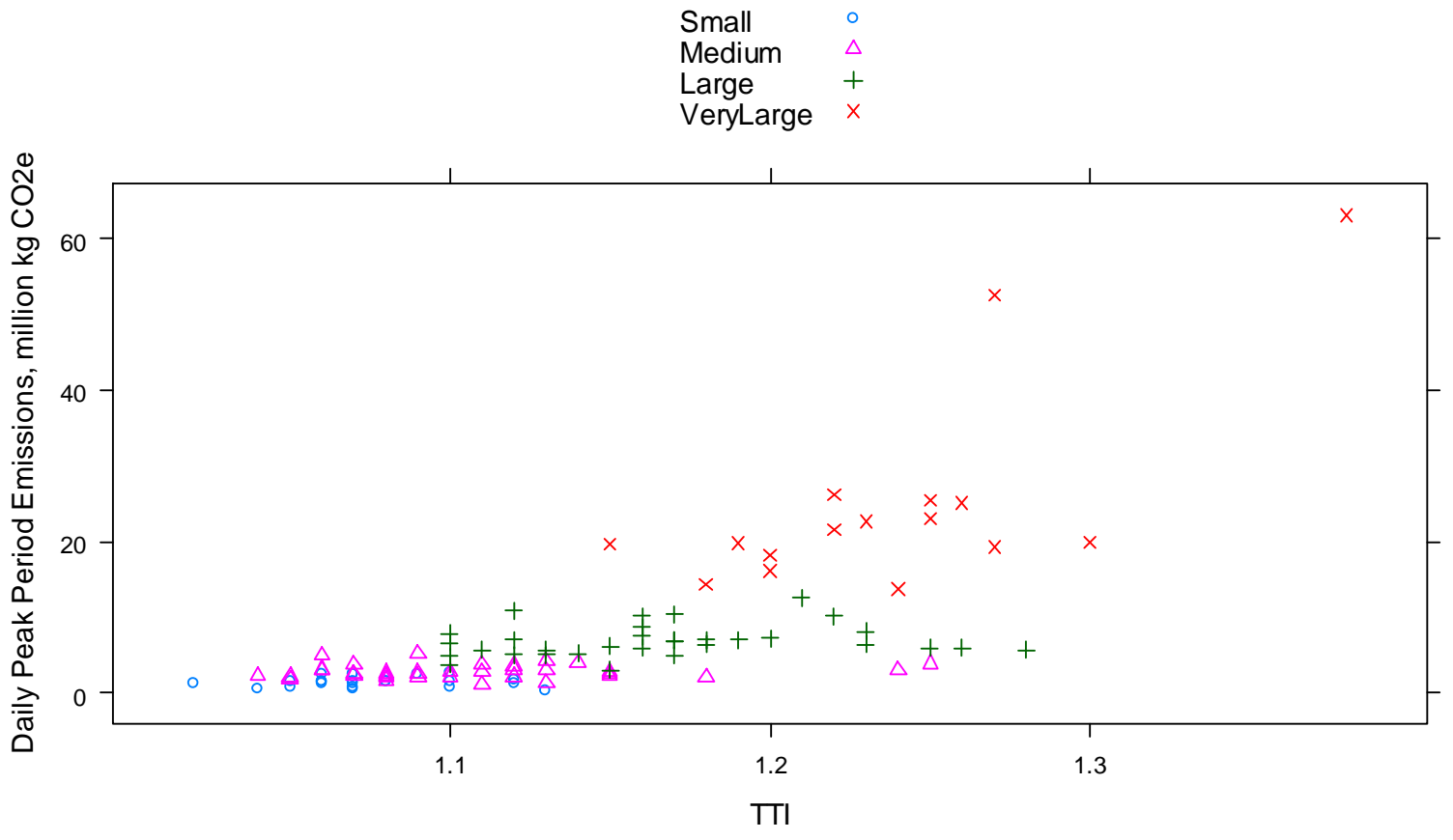

Figure 38. Total Daily Peak-Period $\mathrm{CO}_{2} \mathrm{e}$ Emissions versus TTI, Segmented by Urban Area Population Size

\subsection{Alternative Performance Measures}

As has been pointed out elsewhere, speed-based performance metrics such as the TTI fail to represent the full multi-dimensionality of urban traffic congestion (ECMT, 2007). Cortright (2010) states a need for new macroscopic congestion metrics and offers as alternatives estimates of excess travel distance and excess travel time for urban areas. From the preceding sections, these are immediately more attractive for emissions indicators because they incorporate travel volume (either in the distance or time calculation). In fact, total travel time (unlike delay per unit distance) incorporates both.

As a comparison with the TTI results above, an "excess miles per traveler" metric would have the same strong relationship with emissions per traveler as is shown in Figure 37, but with a horizontal shift equal to the base mileage threshold (Cortright (2010) uses 
16 miles for Large urban areas). Vehicle-hours traveled (VHT) per peak period traveler also has a strong correlation with emissions per traveler, as shown in Figure 39. If compared to some threshold to determine "excess" VHT it would show the same strong correlation, but again with a horizontal shift. Clearly, in terms of reflecting emissions impacts, VMT and VHT are preferable performance indicators to speed or distancenormalized delay.

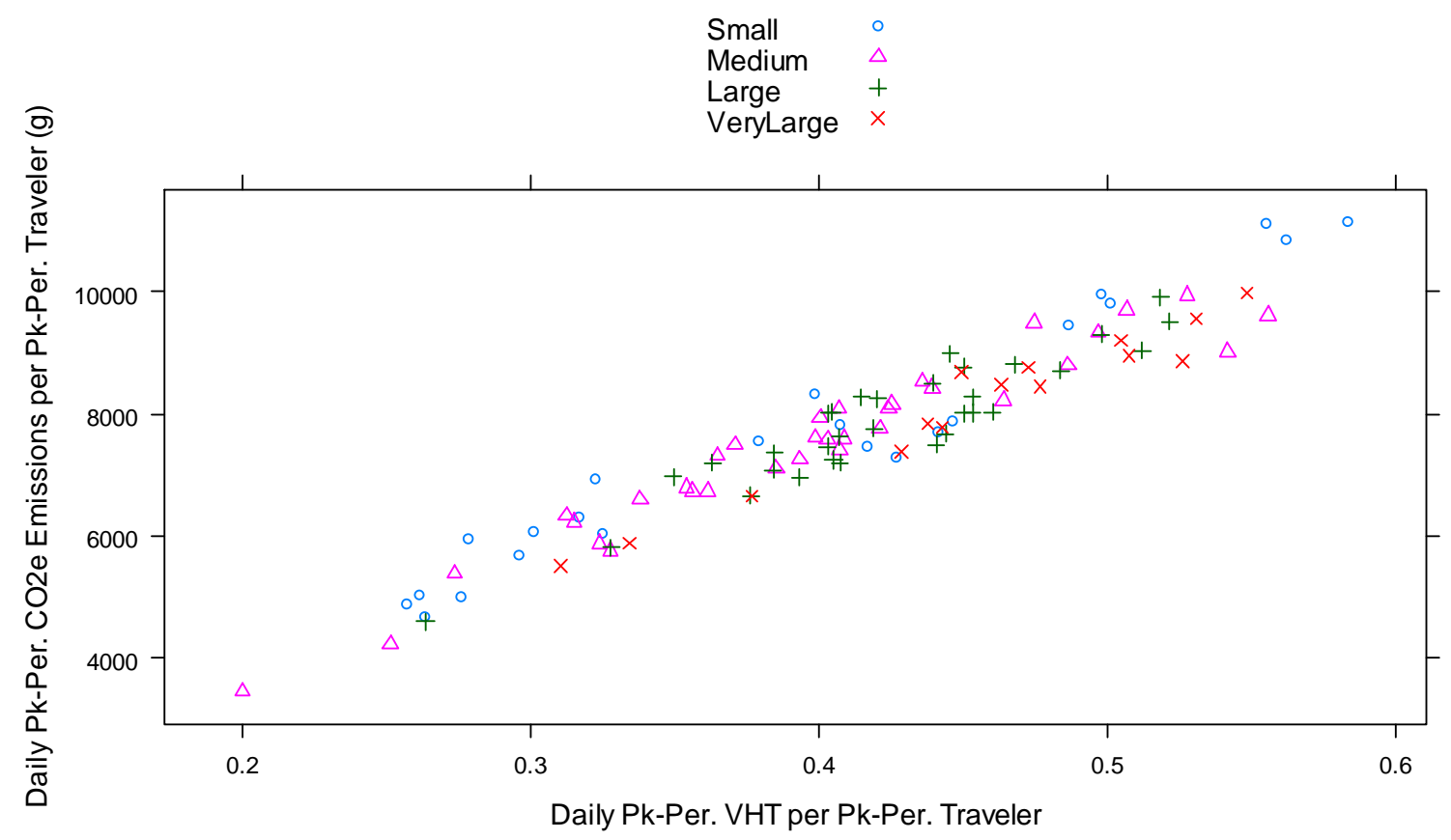

Figure 39. Daily Peak-Period $\mathrm{CO}_{2} \mathrm{e}$ Emissions per Peak-Period Traveler versus Peak-Period VHT per Peak-Period Traveler, Segmented by Urban Area Population Size

Our final comparison of performance measures looks at metropolitan-level changes over time. For Portland, Oregon we calculate TTI, peak period $\mathrm{CO}_{2} \mathrm{e}$ emissions per peak period traveler (as above), peak period travel time per peak period traveler (using $\bar{v}$ and VMT by facility), and peak period VMT per peak period traveler, all with 
the same assumptions as above using the UMR data tables. The emissions rate parameters are fixed at the 2010 value, so we are not assessing the impacts of an evolving vehicle fleet, only the impacts of changing traffic conditions and travel volumes. Figure 40 shows the results for the years 1982-2009, normalized to 1982 values. While emissions, travel time, and VMT all track closely, TTI diverges - in the opposite direction.

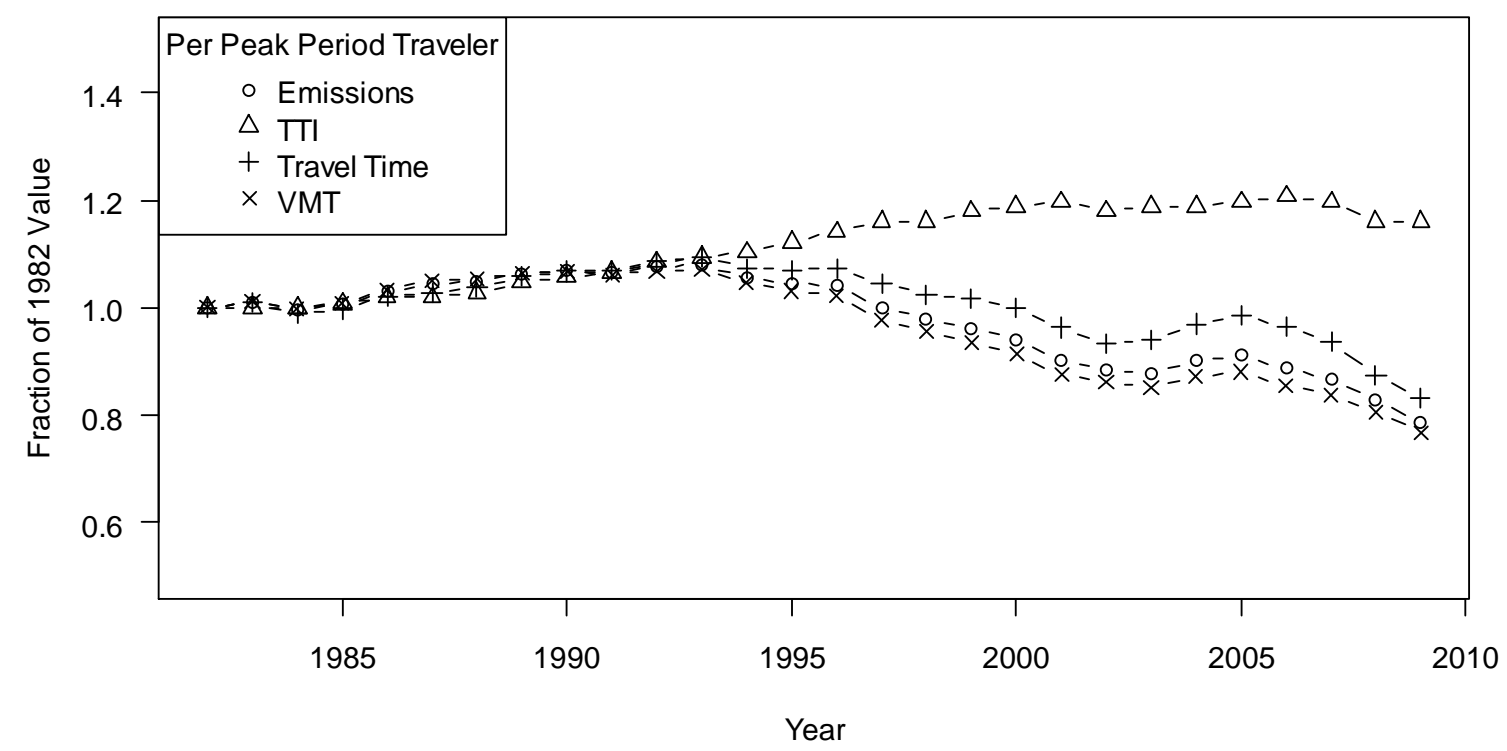

Figure 40. Changes in Performance per Peak-Period Traveler for Portland, Oregon, from 1982 to 2009

We perform a similar comparison for all urban areas in the UMR for the ten-year time interval from 1999 to 2009 in Figure 41. Again, the emissions rate versus speed relationship is taken as static over time in order to isolate traffic impacts. Figure 41 compares changes in TTI, travel time, and VMT per traveler with changes in emissions per traveler for the 10 -year period. In agreement with all preceding results, emissions are much more correlated with VMT and VHT than TTI. These figures show that in addition to the other advantages stated by Cortright (2010), alternative (not delay-based) 
metropolitan-level congestion performance measures are also better indicators of pollution emissions from peak-period travel.
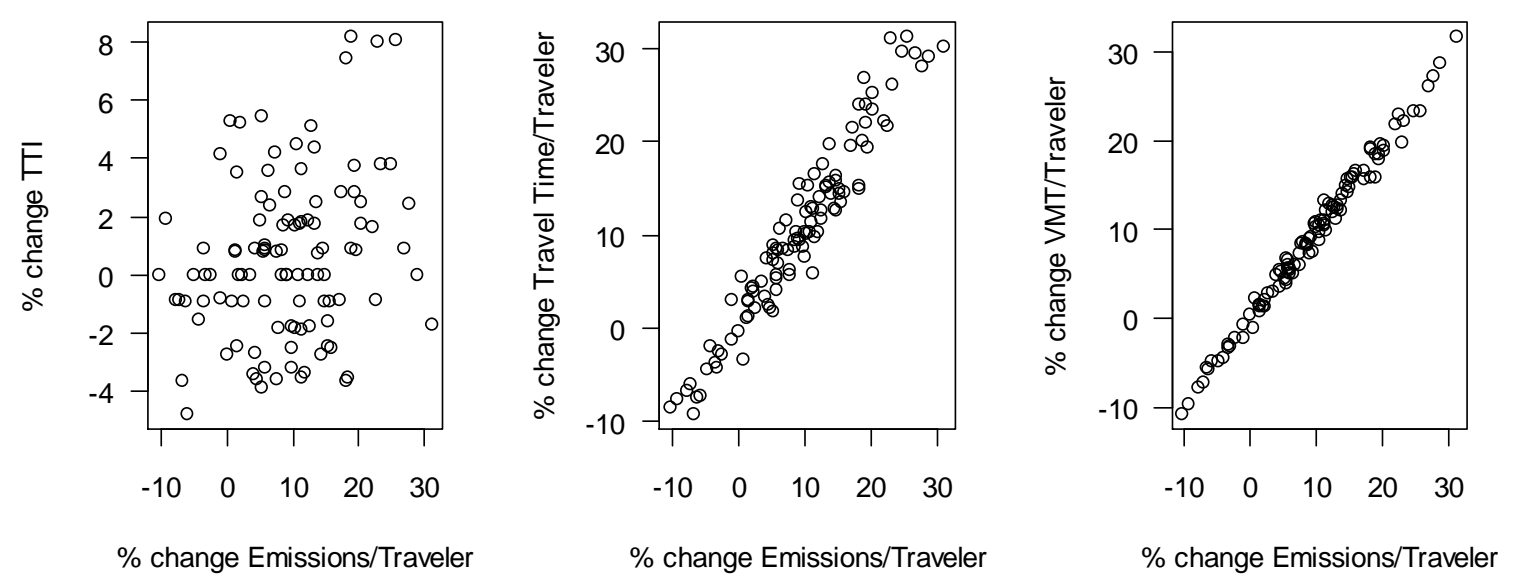

Figure 41. Comparison of Changes in TTI, Travel Time per Peak-Period Traveler, and VMT per Peak-Period Traveler versus Changes in Total Emissions per Peak-

\section{Period Traveler between 1999 to 2009 for all Urban Areas in the UMR}

Because of the strong connection between VMT and total emissions illustrated here, we conclude this section with the suggestion of a framework for looking at the VMT-congestion-emissions connections. Rather than seeing emissions increases as being at the end of a serial path where increasing VMT drives up congestion, which in turn increases emissions, it appears more realistic to view congestion and emissions both as direct, parallel products of increasing VMT. True, heavier congestion levels increase emissions rates per vehicle-mile, but results throughout this thesis demonstrate that in most circumstances travel volume is the dominant factor behind emissions increases, not travel speed. 


\section{CONCLUSIONS}

This thesis represents a step toward better understanding of the potential emissions co-benefits of congestion mitigation. We first presented a unique conceptual framework for addressing the congestion-emissions relationship in a general way. We then developed and applied an original set of sketch-planning equations which generalize the trade-offs between vehicle efficiency and travel demand volume. This fills an important gap in the literature by combining both vehicle efficiency and variable demand effects on emissions.

While the exact relationships among emissions, travel speed, and travel demand vary with location and pollutant, several consistent results arise. First, travel volume is a key consideration for the emissions impacts of congestion or congestion mitigation looking at speed alone only reveals part (and more often the smaller part) of the picture. Second, higher levels of congestion do not necessarily increase emissions, nor will congestion mitigation inevitably reduce emissions. As such, congestion mitigation strategies and efforts cannot automatically claim "green" status. The results presented in this thesis provide quantitative support for the decoupling of congestion and emissions mitigation strategies.

\subsection{Sketch-Planning Equations for Elasticity of Emissions}

In Chapter 4 we developed a set of equations relating traffic speed, travel volume, and vehicle emissions. Those relationships were used in the analysis to calculate the elasticity of emissions to changes in traffic conditions. This set of relatively simple equations can be used by anyone wishing to estimate the emissions impacts of traffic, 
roadway, or demand management strategies without detail. The key parameters needed for their application are emissions-speed curve fit coefficients and total demand elasticity to travel speed or travel time. Emissions-speed curve fit coefficients are provided in Section 5.1 and Section 7.1 for light-duty, heavy-duty, and LD/HD mixed fleets of vehicles in Portland, Oregon in 2010 (based on the MOVES emissions model). Other, distinct locations will need to develop their own emissions-speed curve fits, though this can be done at a regional level. Travel demand elasticity to speed is a more challenging value to estimate, and will most likely require a range of values for calculation of a range of expected emissions impacts. Still, these equations can be useful sketch-planning tools for incorporation of emissions considerations.

\subsection{Emissions Rates}

The central conclusion from the emissions-speed relationship analysis in Chapter 5 is that the potential for marginal emissions rate reductions through average travel speed adjustments is small between about 25 and $70 \mathrm{mph}$. Larger emissions rate reductions are possible by moderating speeds that are outside this range, however, as vehicle efficiency degrades quickly at very high and very low speeds. These results were consistent across emissions models (with some variation in optimal speeds), suggesting that they are also applicable for other locations and vehicle fleets.

The potential for emissions rate reductions by increasing average speeds is greater on arterials than on freeways, mostly because of lower operating speeds. Heavy-duty vehicles have emissions rates that range from roughly equal to those of light-duty vehicles (for $\mathrm{CO}$ ) to 60 times greater (for $\mathrm{PM}_{2.5}$ ). This difference is partly due to the 
dominance of diesel fuel for heavy-duty vehicles. Heavy-duty vehicles are generally more sensitive to low-speed inefficiency as well. As such, greater portions of heavy vehicles in the fleet increase the overall emissions sensitivity to speed changes.

Comparing spatial and temporal marginal emissions rates, we showed that lowspeed inefficiency is only applicable when emissions are normalized to distance, not time. While spatial rates are the most common metric for assessing emissions, this demonstrates that distance/time trade-offs are also important to consider in order to see the full relationship between emissions and speed. Finally, we fit simplified emissions rate curves to traffic volume as the independent variable, making use of the BPR volumespeed function. These curves demonstrate increasing emissions rates with increasing flows, and can be used for traffic modeling which requires simplified and integrated emissions and volume estimation (such emissions-minimizing traffic flow optimization).

\subsection{Total Emissions}

In Section 5.4 we brought together emissions rates and travel volumes to look at total emissions as they relate to traffic speed. Total emissions are influenced by two opposing factors with respect to decreasing travel speeds: generally increasing emissions rates (below some optimal speed in the range of 45 to $65 \mathrm{mph}$, depending on conditions) and decreasing travel demand volume. The direction and magnitude of total emissions changes with traffic speed changes depend on the relative size of each.

The fundamental trade-off for total emissions is between efficiency and volume. For highly elastic travel demand conditions, total emissions will generally increase with speed, whereas for highly elastic emissions rates (at lower average speeds, for example) 
total emissions will tend to decrease with increasing speed. In addition to low-speed conditions, emissions rate elasticity is larger (more negative) for heavy-duty vehicles and certain pollutants $\left(\mathrm{HC}\right.$ and $\left.\mathrm{PM}_{2.5}\right)$. An additional consideration for local pollutants is the location of emissions, since congested segments displace queued vehicle emissions upstream.

To explore the efficiency/volume trade-offs we looked at emissions break-even conditions of average speed and demand elasticity. Total emissions are expected to increase with speed for a wide range of conditions. Depending on the pollutant and vehicle fleet, total emissions are generally only expected to decrease with increasing speed for low demand elasticities and low speeds. The total emissions elasticity is expected to increase with the fraction of heavy-duty vehicles and decrease with the fraction of advanced-drivetrain vehicles in the fleet.

\subsection{Congestion Mitigation}

We also used the preceding equations and results to estimate the impacts of congestion mitigation on emissions. For capacity-based congestion mitigation (including traffic flow improvements), the net emissions effect depends on the balance of induced demand and increased efficiency described above (which, in turn, depend on the pollutants of interest, existing congestion levels, fleet composition, etc.). A key uncertainty in the analysis is the net demand elasticity to speed or travel time changes, which must be estimated locally.

For many conditions, freeway capacity expansions that reduce marginal emissions rates by increasing travel speeds are likely to increase total emissions in the long run 
through induced demand. Arterial roadways, more heavy-duty vehicles in the fleet, and pollutants other than $\mathrm{CO}_{2}$ have greater potential for emissions reductions through traffic flow improvements. However, the amplification of emissions rates in congestion is mitigated with more advanced vehicles in the fleet, such as electric vehicles and gasolineelectric hybrid vehicles. For traffic speed increases above the emissions-optimal speed (most often in the range of 45 to $65 \mathrm{mph}$ ), total emissions are subject to the compounding effects of both lower efficiency and induced demand. Comparing capacity-based congestion mitigation strategies with alternative emissions reduction strategies we see that where emissions reductions are possible through speed increases, the small benefits are likely to be more easily and cost-effectively attained by other strategies.

The high emissions rates, high share of total emissions, and high emissions rate sensitivity to speed of heavy-duty vehicles makes vehicle class-targeted congestion mitigation strategies an attractive option. A sketch analysis of vehicle-class segregated facilities showed that truck-only lane strategies consistently out-perform generalpurpose/mixed-flow lane strategies in terms of emissions reductions. Conversion of a general purpose lane to a truck-only lane produces more emissions benefits than a truckonly lane as additional capacity - and the emissions benefits can be amplified by tolling. For vehicle class-specific strategies, the elasticity of freight demand to travel time is a key consideration, and one which is poorly quantified in the literature. Heavy-duty vehicle travel demand elasticity more generally has a large impact on the potential emissions effects of capacity-based congestion mitigation.

An analysis of several congestion-related performance measures showed that for reflecting emissions impacts, VMT is an essential component of performance. Thus, 
alternative congestion metrics such as total/excess travel distance and travel time are preferable emissions performance indicators to speed or distance-normalized delay. The TTI, in particular, poorly reflects emissions changes on congested roadways.

\subsection{Final Thoughts and Future Work}

In conclusion, congestion mitigation and traffic flow improvements cannot rightly be labeled as emissions-reducing unless travel speeds are low and demand elasticity is slight. This includes projects that seek to increase vehicle throughput from existing roadway supply through better traffic management and operations (signal coordination, ramp metering, etc.). Congestion mitigation through reduced vehicle volumes, on the other hand, presents the opportunity for additive emissions benefits through efficiency improvements and volume reductions. This thesis presents a sketch-modeling method by which the balance of efficiency and volume trade-offs can be assessed.

This is a macroscopic analysis intending to describe the broad relationship between congestion and emissions mitigations in many contexts. It neglects some unique emissions effects of microscopic traffic features and some indirect impacts of congestion. Driver behavior responses to congestion are modeled simply as aggregate travel demand elasticity to travel speed changes. Future work will investigate the impacts of behavior responses in more detail, since different travel demand shifts (changes in mode, departure time, route, destination, etc.) will have differing impacts on the volume of emissions - in addition to the spatial-temporal allocation of emissions. Additional next steps include detailed analysis of the broad emissions impacts of travel time unreliability, freight responses to congestion, distinct traffic flow features such as bottlenecks, and network- 
level travel patterns (which relate back to driver behavior responses). Furthermore, we hope to shortly present a broader analysis of congestion performance metrics as they relate to total social costs (including time, emissions, the economy, and more). 


\section{REFERENCES}

Abdelwahab, W. M. (1998). Elasticities of mode choice probabilities and market elasticities of demand: Evidence from a simultaneous mode choice/shipment-size freight transport model. Transportation Research Part E: Logistics and Transportation Review, 34(4), 257-266. doi:10.1016/S1366-5545(98)00014-3

Adler, K., Grant, M., \& Schroeer, W. (1998). Emissions Reduction Potential of the Congestion Mitigation and Air Quality Improvement Program: A Preliminary Assessment. Transportation Research Record: Journal of the Transportation Research Board, 1641, 81-88.

Affum, J. K., Brown, A. L., \& Chan, Y. C. (2003). Integrating air pollution modelling with scenario testing in road transport planning: the TRAEMS approach. Science of the Total Environment, The, 312(1-3), 1-14.

Anderson, W., Kanaroglou, P., Miller, E., \& Buliung, R. (1996). Simulating Automobile Emissions in an Integrated Urban Model. Transportation Research Record: Journal of the Transportation Research Board, 1520, 71-80. doi:10.3141/1520-09

Bai, S., Nie, Y., \& Niemeier, D. (2007). The impact of speed post-processing methods on regional mobile emissions estimation. Transportation Research Part D:

Transport and Environment, 12(5), 307-324.

Barkenbus, J. N. (2010). Eco-driving: An overlooked climate change initiative. Energy Policy, 38(2), 762-769.

Barlow, T. J., \& Boulter, P. G. (2009). Emissions factors 2009: Report 2 - a review of the average-speed approach for estimating hot exhaust emissions (Research report No. PPR355). UK Department for Transport. Retrieved from 
http://www.dft.gov.uk/pgr/roads/environment/emissions/\#

Barr, L. (2000). Testing for the Significance of Induced Highway Travel Demand in Metropolitan Areas. Transportation Research Record: Journal of the Transportation Research Board, 1706(-1), 1-8. doi:10.3141/1706-01

Barth, M., An, F., Younglove, T., Scora, G., Levine, C., Ross, M., \& Wenzel, T. (2000). Development of a Modal-Emissions Model (No. Project 25-11). NCHRP (p. 435). Transportation Research Board.

Barth, M., \& Boriboonsomsin, K. (2008). Real-World Carbon Dioxide Impacts of Traffic Congestion. Transportation Research Record: Journal of the Transportation Research Board, 2058, 163-171.

Barth, M., \& Boriboonsomsin, K. (2009). Energy and emissions impacts of a freewaybased dynamic eco-driving system. Transportation Research Part D: Transport and Environment, 14(6), 400-410.

Barth, M., Scora, G., \& Younglove, T. (1999). Estimating emissions and fuel consumption for different levels of freeway congestion. Transportation Research Record: Journal of the Transportation Research Board, 1664, 47-57.

Barth, M., Scora, G., \& Younglove, T. (2004). Modal emissions model for heavy-duty diesel vehicles. Transportation Research Record: Journal of the Transportation Research Board, 1880, 10-20.

Beevers, S. D., \& Carslaw, D. C. (2005). The impact of congestion charging on vehicle emissions in London. Atmospheric Environment, 39(1), 1-5.

Bigazzi, A., \& Figliozzi, M. (2011). Freeway Traffic Flow Optimization Considering Delay, Emissions, and Fuel Costs with Stochastic Capacity. In 52nd Annual 
Transportation Research Forum. Long Beach, CA.

Bigazzi, A., Siri, H., \& Bertini, R. (2010). Effects of Temporal Data Aggregation on Performance Measures and other ITS Applications. In 89th Annual Meeting of the Transportation Research Board. Washington, D.C.

Bigazzi, A., Van Lint, J., Klunder, G., Stelwagen, U., \& Ligterink, N. (2010). Traffic data for local emissions monitoring at a signalized intersection. In Intelligent Transportation Systems (ITSC), 2010 13th International IEEE Conference on (pp. 210-215). Presented at the Intelligent Transportation Systems (ITSC), 2010 13th International IEEE Conference on.

Boriboonsomsin, K., \& Barth, M. (2008). Impacts of freeway high-occupancy vehicle lane configuration on vehicle emissions. Transportation Research Part D: Transport and Environment, 13(2), 112-125. doi:10.1016/j.trd.2008.01.001

Boriboonsomsin, K., \& Barth, M. (2007). Evaluating Air Quality Benefits of Freeway High-Occupancy Vehicle Lanes in Southern California. Transportation Research Record: Journal of the Transportation Research Board, 2011, 137-147.

Boulter, P. G., Barlow, T. J., McCrae, I. S., \& Latham, S. (2009). Emissions factors 2009: Final summary report (Research report No. PPR361). UK Department for Transport. Retrieved from http://www.dft.gov.uk/pgr/roads/environment/emissions/\#

Brodrick, C. J., Laca, E. A., Burke, A. F., Farshchi, M., Li, L., \& Deaton, M. (2004). Effect of Vehicle Operation, Weight, and Accessory Use on Emissions from a Modern Heavy-Duty Diesel Truck. Transportation Research Record: Journal of the Transportation Research Board, 1880(-1), 119-125. 
Brownstone, D., \& Small, K. A. (2005). Valuing time and reliability: assessing the evidence from road pricing demonstrations. Transportation Research Part A: Policy and Practice, 39(4), 279-293. doi:10.1016/j.tra.2004.11.001

Bureau of Public Roads. (1964). Traffic Assignment Manual. U.S. Department of Commerce.

Cambridge Systematics, Inc., \& Texas Transportation Institute. (2005). Traffic Congestion and Reliability: Trends and Advanced Strategies for Congestion Mitigation. Washington, D.C.: Federal Highway Administration.

Cervero, R. (2002). Induced Travel Demand: Research Design, Empirical Evidence, and Normative Policies. Journal of Planning Literature, 17(1), 3 -20. doi:10.1177/088122017001001

Chen, H., Namdeo, A., \& Bell, M. (2008). Classification of road traffic and roadside pollution concentrations for assessment of personal exposure. Environmental Modelling and Software, 23(3), 282-287.

Chen, K., \& Yu, L. (2007). Microscopic traffic-emission simulation and case study for evaluation of traffic control strategies. Journal of Transportation Systems Engineering and Information Technology, 7(1), 93-99.

Chu, H., \& Meyer, M. D. (2009). Methodology for assessing emission reduction of truckonly toll lanes. Energy Policy, 37(8), 3287-3294.

Cohen, H. S. (1995). Expanding metropolitan highways: Implications for air quality and energy use. Appendix B: Review of empirical studies of induced traffic. Transportation Research Board Special Report, (245).

Cortright, J. (2010). Measuring Urban Transportation Performance: a Critique of 
Mobility Measures and a Synthesis. CEO's for Cities.

Croxford, B., \& Penn, A. (1998). Siting considerations for urban pollution monitors. Atmospheric Environment, 32(6), 1049-1058.

DeCorla-Souza, P., \& Cohen, H. (1999). Estimating induced travel for evaluation of metropolitan highway expansion. Transportation, 26(3), 249-262.

Dijkema, M. B., van der Zee, S. C., Brunekreef, B., \& van Strien, R. T. (2008). Air quality effects of an urban highway speed limit reduction. Atmospheric Environment, 42(40), 9098-9105.

Dowling, R., Skabardonis, A., Carroll, M., \& Wang, Z. (2004). Methodology for measuring recurrent and nonrecurrent traffic congestion. Transportation Research Record: Journal of the Transportation Research Board, 1867(-1), 60-68.

Dowling, R. G. (2005). Predicting air quality effects of traffic-flow improvements: final report and user's guide (NCHRP No. 535). Transportation Research Board.

Ericsson, E. (2000). Variability in urban driving patterns. Transportation Research Part $D, 5(5), 337-354$.

European Conference of Ministers of Transport (ECMT). (2007). Managing Urban Traffic Congestion. OECD, Transport Research Center.

Farzaneh, M., Schneider, W., \& Zietsman, J. (2010). Field Evaluation of Carbon Dioxide Emissions at High Speeds. In 89th Annual Meeting of the Transportation Research Board. Washington, D.C.

Federal Highway Administration. (2010, February 16). Congestion Mitigation and Air Quality (CMAQ) Improvement Program. Retrieved February 16, 2010, from http://www.fhwa.dot.gov/environment/cmaqpgs/ 
Federal Highway Administration. (2001). Traffic Monitoring Guide (No. FHWA-PL-01021). Washington, D.C.: U.S. Department of Transportation.

Fenger, J. (1999). Urban air quality. Atmospheric Environment, 33(29), 4877-4900.

Figliozzi, M. A. (2011). The impacts of congestion on time-definitive urban freight distribution networks $\mathrm{CO} 2$ emission levels: Results from a case study in Portland, Oregon. Transportation Research Part C: Emerging Technologies, 19(5), 766778. doi:10.1016/j.trc.2010.11.002

Frey, H. C., \& Zheng, J. (2002). Probabilistic analysis of driving cycle-based highway vehicle emission factors. Environ. Sci. Technol, 36(23), 5184-5191.

Goodwin, P. (2004). The economic costs of road traffic congestion (Discussion Paper). London, UK: The Rail Freight Group.

Goodwin, P. (1996). Empirical evidence on induced traffic. Transportation, 23(1). doi:10.1007/BF00166218

Goodwin, P., Dargay, J., \& Hanly, M. (2004). Elasticities of road traffic and fuel consumption with respect to price and income: a review. Transport Reviews, 24(3), 275-292.

Goodwin, P. B. (1992). A Review of New Demand Elasticities with Special Reference to Short and Long Run Effects of Price Changes. Journal of Transport Economics and Policy, 26(2), 155-169.

Graham, D. J., \& Glaister, S. (2004). Road traffic demand elasticity estimates: a review. Transport Reviews, 24(3), 261-274.

Grant, M., Kuzmyak, R., Shoup, L., Hsu, E., Krolik, T., \& Ernst, D. (2008). SAFETEALU 1808: CMAQ Evaluation and Assessment, Phase I Final Report (No. FHWA- 
HEP-08-019). Washington, D.C.: Federal Highway Administration.

Greenwood, I. D., Dunn, R. C. M., \& Raine, R. R. (2007). Estimating the effects of traffic congestion on fuel consumption and vehicle emissions based on acceleration noise. Journal of Transportation Engineering, 133, 96.

Hall, J. V., Brajer, V., \& Lurmann, F. W. (2008). Measuring the gains from improved air quality in the San Joaquin Valley. Journal of Environmental Management, 88(4), 1003-1015. doi:10.1016/j.jenvman.2007.05.002

Hallenbeck, M. E., Ishimaru, J., \& Nee, J. (2003). Measurement of recurring versus nonrecurring congestion. Washington State Transportation Center (TRAC).

Hansen, S., Byrd, A., Delcambre, A., Rodriguez, A., Matthews, S., \& Bertini, R. L. (2005). Using Archived ITS Data to Improve Regional Performance Measurement and Travel Demand Forecasting. Presented at the 2005 CITE Quad/WCTA Regional Conference, Vancouver, B.C., Canada.

HDR. (2009). Assessing the Full Costs of Congestion on Surface Transportation Systems and Reducing Them through Pricing. U.S. Department of Transportation.

Health Effects Institute. (2010). Traffic-Related Air Pollution: A Critical Review of the Literature on Emissions, Exposure, and Health Effects (Special Report No. Special Report 17). Health Effects Institute.

Hirschmann, K., \& Fellendorf, M. (2010). A toolbox to quantify emission reductions due to signal control. In 89th Annual Meeting of the Transportation Research Board. Presented at the 89th Annual Meeting of the Transportation Research Board, Washington, D.C.

HLB Decision Economics Inc. (2008). Freight Benefit/Cost Study: Phase III - Analysis 
of Regional Benefits of Highway-Freight Improvements (No. FHWA-HOP-08019). Washington, D.C.: Federal Highway Administration.

Holmen, B., Sentoff, K., Robinson, M., \& Montane, P. (2010). The University of Vermont TOTEMS Instrumentation Package for Real-World, On-Board Tailpipe Emissions Monitoring of Conventional and Hybrid Light-Duty Vehicles. Presented at the 89th Annual Meeting of the Transportation Research Board, Washington, D.C.

Hymel, K. M., Small, K. A., \& Dender, K. V. (2010). Induced demand and rebound effects in road transport. Transportation Research Part B: Methodological, 44(10), 1220-1241. doi:10.1016/j.trb.2010.02.007

Jackson, E., \& Aultman-Hall, L. (2010). Analysis of Real-World Lead Vehicle Operation for the Integration of Modal Emissions and Traffic Simulation Models. Presented at the 89th Annual Meeting of the Transportation Research Board, Washington, D.C.

Johansson, O. (1997). Optimal road-pricing: simultaneous treatment of time losses, increased fuel consumption, and emissions. Transportation Research Part D, 2(2), 77-87.

Jong, G. D., \& Gunn, H. (2001). Recent Evidence on Car Cost and Time Elasticities of Travel Demand in Europe. Journal of Transport Economics and Policy, 35(2), 137-160.

Joumard, R., Philippe, F., \& Vidon, R. (1999). Reliability of the current models of instantaneous pollutant emissions. Science of the Total Environment, The, 235(13), 133-142. 
Kohler, M., Corsmeier, U., Vogt, U., \& Vogel, B. (2005). Estimation of gaseous realworld traffic emissions downstream a motorway. Atmospheric Environment, 39(31), 5665-5684.

Kriger, D., Miller, C., Baker, M., \& Joubert, F. (2007). Costs of Urban Congestion in Canada: A Model-Based Approach. Transportation Research Record: Journal of the Transportation Research Board, 1994, 94-100.

Krimmer, M., \& Venigalla, M. (2006). Measuring impacts of high-occupancy-vehicle lane operations on light-duty-vehicle emissions: Experimental study with instrumented vehicles. In Air Quality 2006, Transportation Research Record (pp. 1-10). Washington, DC: National Research Council.

Kuhlwein, J., \& Friedrich, R. (2000). Uncertainties of modelling emissions from road transport. Atmospheric Environment, 34(27), 4603-4610.

Kuhlwein, J., \& Friedrich, R. (2005). Traffic measurements and high-performance modelling of motorway emission rates. Atmospheric Environment, 39(31), 57225736.

Kwon, J., Mauch, M., \& Varaiya, P. (2006). Components of Congestion: Delay from Incidents, Special Events, Lane Closures, Weather, Potential Ramp Metering Gain, and Excess Demand. Transportation Research Record: Journal of the Transportation Research Board, 1959, 84-91.

Lee, D., Klein, L., \& Camus, G. (1999). Induced Traffic and Induced Demand. Transportation Research Record: Journal of the Transportation Research Board, 1659(-1), 68-75. doi:10.3141/1659-09

Lin, J., \& Niemeier, D. (2003a). Estimating Regional Air Quality Vehicle Emission 
Inventories: Constructing Robust Driving Cycles. TRANSPORTATION SCIENCE, 37(3), 330-346. doi:10.1287/trsc.37.3.330.16045

Lin, J., \& Niemeier, D. (2003b). Regional driving characteristics, regional driving cycles. Transportation Research Part D: Transport and Environment, 8(5), 361-381. doi:10.1016/S1361-9209(03)00022-1

Lin, J., \& Niemeier, D. (2002). An exploratory analysis comparing a stochastic driving cycle to California's regulatory cycle. Atmospheric Environment, 36(38), 57595770. doi:10.1016/S1352-2310(02)00695-7

Litman, T. (2011). Transportation Elasticities: How Prices and Other Factors Affect Travel Behavior. Victoria Transport Policy Institute. Retrieved from http://www.google.com/search?ie=UTF-8\&oe=UTF$8 \&$ sourceid=navclient $\&$ gfns $=1 \& q=$ travel + demand + elasticity + to + travel + time Mahmod, M., van Arem, B., Pueboobpaphan, R., \& Igamberdiev, M. (2010). Modeling reduced traffic emissions in urban areas: the impact of demand control, banning heavy duty vehicles, speed restriction and adaptive cruise control. Presented at the 89th Annual Meeting of the Transportation Research Board, Washington, D.C.

Malcolm, C., Younglove, T., Barth, M., \& Davis, N. (2003). Mobile-Source Emissions: Analysis of Spatial Variability in Vehicle Activity Patterns and Vehicle Fleet Distributions. Transportation Research Record: Journal of the Transportation Research Board, 1842, 91-98.

May. (1989). Traffic Flow Fundamentals. Prentice Hall.

Metz, D. (2008). The myth of travel time saving. Transport Reviews, 28(3), 321-336. Middleton, D. (2006). Strategies for Separating Trucks from Passenger Vehicles: Truck 
Facility Guidebook. Texas Transportation Institute, Texas A\&M University System.

Nagurney, A. (2000). Congested urban transportation networks and emission paradoxes. Transportation Research Part D: Transport and Environment, 5(2), 145-151. doi:10.1016/S1361-9209(99)00031-0

Nesamani, K. S., \& Subramanian, K. P. (2006). Impact of Real-world Driving Characteristics on Vehicular Emissions. JSME International Journal Series B, 49(1), 19-26.

Noland, R. B. (2001). Relationships between highway capacity and induced vehicle travel. Transportation Research Part A: Policy and Practice, 35(1), 47-72.

Noland, R. B., \& Cowart, W. A. (2000). Analysis of metropolitan highway capacity and the growth in vehicle miles of travel. Transportation, 27(4), 363-390.

Noland, R. B., \& Lem, L. L. (2002). A review of the evidence for induced travel and changes in transportation and environmental policy in the US and the UK. Transportation Research Part D, 7(1), 1-26.

Noland, R. B., \& Quddus, M. A. (2006). Flow improvements and vehicle emissions: Effects of trip generation and emission control technology. Transportation Research Part D, 11(1), 1-14.

Oregon Department of Transportation. (2010, August 24). Traffic Volumes and Vehicle Classification. Retrieved November 3, 2010, from http://highway.odot.state.or.us/cf/highwayreports/traffic_parms.cfm

Oum, T. H. (1989). Alternative demand models and their elasticity estimates. Journal of Transport Economics and Policy, 23(2), 163-187. 
Oum, T. H., II, W. G. W., \& Yong, J. (1992). Concepts of Price Elasticities of Transport Demand and Recent Empirical Estimates: An Interpretative Survey. Journal of Transport Economics and Policy, 26(2), 139-154.

Pandian, S., Gokhale, S., \& Ghoshal, A. K. (2009). Evaluating effects of traffic and vehicle characteristics on vehicular emissions near traffic intersections. Transportation Research Part D, 14(3), 180-196.

Park, J. Y., Noland, R. B., \& Polak, J. W. (2001). Microscopic Model of Air Pollutant Concentrations: Comparison of Simulated Results with Measured and Macroscopic Estimates. Transportation Research Record: Journal of the Transportation Research Board, 1750, 64-73.

Park, S., Rakha, H., Farzaneh, M., Zietsman, J., \& Lee, D. (2010). Development of Fuel and Emission Models for High Speed Heavy Duty Trucks, Light Duty Trucks, and Light Duty Vehicles. In Proceedings of the 13th International IEEE Conference on ITS. Presented at the 13th International IEEE Conference on Intelligent Transportation Systems, Madeira Island, Portugal.

Rakha, H., Van Aerde, M., Ahn, K., \& Trani, A. (2000). Requirements for Evaluating Traffic Signal Control Impacts on Energy and Emissions Based on Instantaneous Speed and Acceleration Measurements. Transportation Research Record: Journal of the Transportation Research Board, 1738, 56-67. doi:10.3141/1738-07

Raus, J. (1981). A method for estimating fuel consumption and vehicle emissions on urban arterials and networks (No. FHWA-TS-81-210). Federal Highway Administration, Washington, DC (USA).

Roberts, C. A., Washington, S., \& Leonard II, J. D. (1999). Forecasting dynamic 
vehicular activity on freeways: Bridging the gap between travel demand and emerging emissions models. Transportation Research Record: Journal of the Transportation Research Board, 1664, 31-39.

Ropkins, K., Beebe, J., Li, H., Daham, B., Tate, J., Bell, M., \& Andrews, G. (2009). Real-World Vehicle Exhaust Emissions Monitoring: Review and Critical Discussion. Critical Reviews in Environmental Science and Technology, 39(2), $79-152$.

Samaras, C., \& Meisterling, K. (2008). Life Cycle Assessment of Greenhouse Gas Emissions from Plug-in Hybrid Vehicles: Implications for Policy. Environmental Science \& Technology, 42(9), 3170-3176.

Schrank, D., \& Lomax, T. (2007). The 2007 urban mobility report. Texas Transportation Institute, College Station, TX.

Schrank, D., Lomax, T., \& Turner, S. (2010). Urban Mobility Report 2010. College Station, TX: Texas Transportation Institute.

Scora, G., Boriboonsomsin, K., \& Barth, M. (2010). Effects of Operational Variability on Heavy-Duty Truck Greenhouse Gas Emissions. In 89th Annual Meeting of the Transportation Research Board. Washington, D.C.

Singh, R. B., Huber, A. H., \& Braddock, J. N. (2007). Sensitivity analysis and evaluation of microFacPM: A microscale motor vehicle emission factor model for particulate matter emissions. Journal of the Air and Waste Management Association, 57(4), 420-433.

Skabardonis, A., Varaiya, P., \& Petty, K. F. (2003). Measuring recurrent and nonrecurrent traffic congestion. Transportation Research Record: Journal of the 
Transportation Research Board, 1856, 118-124.

Small, K. A., Winston, C., \& Yan, J. (2005). Uncovering the Distribution of Motorists' Preferences for Travel Time and Reliability. Econometrica, 73(4), 1367-1382. doi:10.1111/j.1468-0262.2005.00619.x

Smit, R., Brown, A. L., \& Chan, Y. C. (2008). Do air pollution emissions and fuel consumption models for roadways include the effects of congestion in the roadway traffic flow? Environmental Modelling and Software, 23(10-11), 1262 1270.

Smit, R., Smokers, R., \& Rabe, E. (2007). A new modelling approach for road traffic emissions: VERSIT+. Transportation research. Part D, Transport and environment, 12(6), 414-422.

Smyth, A., \& Christodoulou, G. (2010). Congestion Relief: Have We the Stomach for The Remedies? Presented at the 89th Annual Meeting of the Transportation Research Board, Washington, D.C.

Stathopoulos, F. G., \& Noland, R. B. (2003). Induced travel and emissions from traffic flow improvement projects. Transportation Research Record: Journal of the Transportation Research Board, 1842, 57-63.

Stopher, P. R. (2004). Reducing road congestion: a reality check. Transport Policy, 11(2), 117-131.

Sugawara, S., \& Niemeier, D. (2002). How much can vehicle emissions be reduced? Exploratory analysis of an upper boundary using an emissions-optimized trip assignment. Transportation Research Record, 1815, 29-37.

Transportation Research Board. (2002). The Congestion Mitigation and Air Quality 
Improvement Program: Assessing 10 Years of Experience (No. Special Report 264). Washington, D.C.: National Academies.

Transportation Research Board. (2000). Highway Capacity Manual. Washington, D.C.: National Research Council.

Transportation Research Board. (2010). Separation of Vehicles - CMV-Only Lanes (NCHRP No. 649). Washington, D.C.: National Academies.

Tu, H., van Lint, J., \& van Zuylen, H. (2007). Impact of Traffic Flow on Travel Time Variability of Freeway Corridors. Transportation Research Record: Journal of the Transportation Research Board, 1993, 59-66. doi:10.3141/1993-09

U.S. Environmental Protection Agency (EPA). (2009a). Inventory of U.S. Greenhouse Gas Emissions and Sinks: 1990-2007. Retrieved from http://www.epa.gov/climatechange/emissions/usinventoryreport.html

U.S. Environmental Protection Agency. (n.d.). AirData | US EPA. Retrieved March 25, 2011, from http://www.epa.gov/air/data/index.html

U.S. Environmental Protection Agency. (2009c). Motor Vehicle Emission Simulator (MOVES) 2010 User's Guide (No. EPA-420-B-09-041) Washington D.C. Retrieved from http://www.epa.gov/otaq/models/moves/index.htm

U.S. Environmental Protection Agency. (2009b). Light-Duty Automotive Technology, Carbon Dioxide Emissions, and Fuel Economy Trends: 1975 Through 2009 (No. EPA420-S-09-001). Washington, D.C.

U.S. Environmental Protection Agency. (2010). MOVES2010 Highway Vehicle Population and Activity Data (No. EPA-420-R-10-026). U.S. Environmental Protection Agency. Retrieved from 
http://www.epa.gov/otaq/models/moves/420r10026.pdf

Venkatram, A. (2004). On estimating emissions through horizontal fluxes. Atmospheric Environment, 38(9), 1337-1344.

Venkatram, A., Isakov, V., Thoma, E., \& Baldauf, R. (2007). Analysis of air quality data near roadways using a dispersion model. Atmospheric Environment, 41(40), 9481-9497.

Wang, Z., \& Liu, C. (2005). An empirical evaluation of the loop detector method for travel time delay estimation. Journal of Intelligent Transportation Systems, 9(4), $161-174$.

Weisbrod, G., Vary, D., \& Treyz, G. (2001). NCHRP Report 463: Economic Implications of Congestion. Washington, D.C.: Transportation Research Board.

Williams, H. C. W. L., \& Moore, L. A. R. (1990). The Appraisal of Highway Investments under Fixed and Variable Demand. Journal of Transport Economics and Policy, 24(1), 61-81.

Wu, G., Boriboonsomsin, K., Zhang, W., Li, M., \& Barth, M. (2010). Energy and Emission Benefit Comparison between Stationary and In-Vehicle Advanced Driving Alert Systems. Presented at the 89th Annual Meeting of the Transportation Research Board, Washington, D.C.

Yun, S., White, W., Lamb, D., \& Wu, Y. (2005). Accounting for the Impact of Heavy Truck Traffic in Volume-Delay Functions in Transportation Planning Models. Transportation Research Record: Journal of the Transportation Research Board, 1931(-1), 8-17. doi:10.3141/1931-02

Zegeye, S. K., De Schutter, B., Hellendoorn, H., \& Breunesse, E. (2009). Reduction of 
Travel Times and Traffic Emissions Using Model Predictive Control. American Control Conference.

Zietsman, J., \& Rilett, L. (2001). Analysis of Aggregation Effects in Vehicular Emission Estimation. Transportation Research Record: Journal of the Transportation Research Board, 1750, 56-63. doi:10.3141/1750-07 


\section{APPENDIX A: MAJOR STUDIES OF THE IMPACTS OF CONGESTION ON EMISSIONS}

\section{TEXAS TRANSPORTATION INSTITUTE'S URBAN MOBILITY REPORT}

The annual Urban Mobility Report (UMR) is an ongoing assessment of congestion in U.S. cities that reports a set of performance measures including the extent of congestion, traveler delay, "wasted" fuel, and congestion costs (in dollars) (Schrank \& Lomax, 2009). Emissions are not explicitly included in the assessment, though fuel consumption is (a rough predictor of greenhouse gas emissions). The fuel consumption is estimated from a simple linear regression equation using average system travel speed. The fuel regression equation comes from a U.S. Federal Highway Administration report published in 1981 (Raus, 1981), and its use has been criticized because it was only intended for application up to $35 \mathrm{mph}$ (HDR, 2009). The benchmark for comparison in the UMR is free-flow travel speeds (assumed $60 \mathrm{mph}$ freeway, $35 \mathrm{mph}$ arterial). "Wasted" fuel is then the difference between fuel consumption at free-flow speeds and at actual speeds, which considers most factors to be exogenous (including auto travel).

\section{ECMT: MANAGING URBAN TRAFFIC CONGESTION}

This large study presents a thorough analysis and discussion of the full extent and characteristics of urban traffic congestion in Europe (European Conference of Ministers of Transport (ECMT), 2007). The complexities of and barriers to estimating emissions impacts are discussed. Although no quantitative emissions estimates are made, the report suggests that fuel consumption and 'environmental pollution' will increase with congestion. 
The conclusion with respect to estimating air pollution is that in addition to average travel speed, microscopic flow characteristics must be considered. Also, the report makes the case (supported by Goodwin (2004)) that congestion cost estimates based on free-flow reference speeds are "artificial constructs," and should not be used. Two other assertions, also claimed by Stopher (2004), are that congestion cannot be eliminated, only managed, and that in the same way flow improvements induce demand, congestion suppresses it. These perspectives support the notion that congestion effects arbitrarily benchmarked to fixed-demand free-flow conditions are not relevant.

\section{U.S. DEPARTMENT OF TRANSPORTATION'S CONGESTION MANAGEMENT AND AIR QUALITY (CMAQ) IMPROVEMENT PROGRAM}

The CMAQ program was authorized by the U.S. Congress as part of the ISTEA surface transportation act in 1991, and has since been administered by the U.S. Department of Transportation (Federal Highway Administration, 2010). Since its inception the program has provided over $\$ 14$ billion in federal funding to support transportation projects that "contribute to air quality improvements and reduce congestion." But these objectives are not necessarily mutually beneficial, as has been pointed out by others (Noland \& Quddus, 2006). Specifically, traffic flow improvements (33\% of CMAQ projects) can induce travel demand that cancels any short-term emissions reductions (Noland \& Quddus, 2006; Stathopoulos \& Noland, 2003).

An early assessment of the program by Adler et al. estimated small emissions benefits for most CMAQ projects, but noted trade-offs among different pollutants (especially for $\mathrm{NO}_{\mathrm{x}}$ ) (1998). Adler et al. also reported high uncertainty in estimated 
emissions benefits, a distinct lack of emissions reporting standards, and induced demand unaccounted for. The execution of this program highlights the need for better understanding of how congestion impacts roadway emissions.

\section{ASSESSING THE FULL COSTS OF CONGESTION ON SURFACE TRANSPORTATION SYSTEMS AND REDUCING THEM THROUGH PRICING}

A large modeling effort was recently undertaken by HDR for the Office of Economic and Strategic Analysis in the U.S. Department of Transportation (HDR, 2009). This study estimates congestion costs in U.S. cities using a macroscopic, average-speed approach, with an aim of assessing potential benefits of congestion pricing. The benchmark for emissions estimates, similar to the UMR, is free-flow conditions (fixed travel, steady speeds). In estimating pricing effects, travel demand elasticity to generalized cost was used (which did include travel time unreliability). Demand elasticity was not, however, incorporated into the congestion cost estimates.

Emissions costs comprise less than $1 \%$ of total estimated congestion costs (which include travel time, unreliability, vehicle operating costs, and mobility), and are negative (indicating benefits) for some cities. The study's macroscopic approach does not consider detailed flow characteristics or some higher-order effects, but shows that even averagespeed comparisons can suggest increased or decreased emissions during congestion, depending on conditions. The report concludes that "vehicle emissions contribute negligibly to the costs of congestion," and that road improvements can increase emissions because of high emissions at free-flow speeds and induced demand. 


\section{NCHRP REPORT 535: PREDICTING AIR QUALITY EFFECTS OF TRAFFIC- FLOW IMPROVEMENTS}

This report addresses congestion mitigation, not cost quantification, but does focus on estimating emissions with changes in congestion (Dowling, 2005). The study objective was a methodology, not quantification, although a few case studies were performed. The report describes a detailed travel demand modeling approach that includes predictions of travel demand and growth pattern responses to travel time reductions. As expected for a methodology of this scope, validation was limited.

The main strength of the method is the inclusion of long-term, higher-order effects such as land use changes. However, the methodology represents capacity and travel-time improvements, so microscopic traffic flow changes cannot be modeled. Also, while travel demand is elastic with respect to total travel time, demand is insensitive to travel time reliability.

A small set of ten case studies were performed for traffic flow improvements in the Seattle/Tacoma metropolitan area. Overall, the modeled flow improvements had almost no effect on total regional travel which would indicate induced demand. However, Noland and Quddus (2006) point out that the small scale of the projects and large uncertainties in the model highlight the weaknesses of these macroscopic modeling approaches. The final conclusion from the case studies is that more research is needed "to better understand the conditions under which traffic-flow improvements contribute to an overall net increase or decrease in vehicle emissions." 


\section{MICROSCOPIC STUDY OF INDUCED DEMAND FROM FLOW IMPROVEMENTS}

In contrast to the macroscopic modeling undertaken by Dowling (2005) to estimate the emissions effects of flow improvements, researchers in London have taken a microscopic approach. In separate papers Stathopoulos and Noland (2003) and Noland and Quddus (2006) used traffic micro-simulation and microscopic emissions models to calculate emissions 'break-even' demand elasticity to travel time (the point at which induced demand cancels the emissions benefits of improved flow) for each pollutant. The scenarios considered were signal coordination and lane expansion on an arterial and a freeway. Both studies concluded that long-run emissions reductions were unlikely, as the break-even demand elasticities for short time horizons were well within the range of published values.

The emissions effects varied with pollutant and vehicle fleet. In particular, Noland and Quddus found that the emissions savings from improving flow characteristics were negligible for modern, cleaner vehicles (for hydrocarbons and carbon monoxide). In contrast, older and dirtier vehicles can have more benefits from flow improvements. As with most other studies, demand elasticity to travel time reliability was not considered. Stathopoulos and Noland assert that these and other studies show the potential emissions benefits of traffic suppression through capacity reductions. The same logic extends to the costs of congestion, where traffic suppression can compensate for the increased emissions due to inefficient driving. 


\section{OTHER TARGETED RESEARCH}

Other papers have also addressed the congestion-emissions relationship directly. Researchers at the University of California, Riverside have investigated the emissions effects of detailed traffic characteristics. These studies generally address short-term effects, where demand and higher-order impacts are not considered. Key findings are increased emissions at high and low average travel speeds, increased emissions of realworld transient driving (as opposed to steady-state), and the importance of short speed fluctuations at high speeds (Barth et al., 1999; Barth \& Boriboonsomsin, 2008).

In particular, Barth, Scora, and Younglove (1999) show that free-flow conditions can have higher emissions (per vehicle-mile) than lower levels of service (for pollutants other than carbon dioxide). Also, Barth and Boriboonsomsin (2009) show that more efficient driving on freeways can reduce greenhouse gas emissions by $10-20 \%$ without a significant change in travel time, with more benefits at higher levels of congestion. This result illustrates the possibilities of congestion management that reduces emissions (through more efficient driving behavior) without inducing demand.

In other work, a study by Greenwood, Dunne, and Raine (2007) showed 12-25\% increases in emissions for congested driving on urban arterials. The reference case was 30 $\mathrm{km} / \mathrm{hr}$ steady-state driving, and higher-order effects were not considered. Modeling of traffic control on arterials by Zegeye et al. (2009) showed that both travel time and emissions cannot be minimized, and optimization involves some trade-off between the two. An interesting paper by Nagurney (2000) illustrates 3 paradoxes of the congestionemissions relationship at the network level. They are essentially network assignment scenarios in which network improvements lead to increases in emissions, with the 
cautionary conclusion that the "network topology, the demand structure, as well as the link travel cost structure must all be incorporated into any environmental modeling."

Finally, Beevers and Carslaw (2005) studied air quality in London after a pricing scheme severely reduced traffic flow in the city center. They found $12 \%$ reductions in $\mathrm{NO}_{\mathrm{x}}$ and particulates, with larger savings from improving travel speeds than decreasing flows. The reduced personal vehicle emissions were partly offset by increased transit bus mileage as a result of mode shift. 